\title{
Materie-Optik mit Edelgasmolekülen an Nanostrukturen
}

\author{
Dissertation \\ zur Erlangung des Doktorgrades \\ der Mathematisch-Naturwissenschaftlichen Fakultäten \\ der Georg-August-Universität zu Göttingen \\ vorgelegt von \\ Werner Martin Stoll \\ aus Peine
}

Göttingen 2003 
D7

Referent: Prof. Dr. G. C. Hegerfeldt

Korreferent: Prof. Dr. K. Schönhammer

Tag der mündlichen Prüfung: 18.12.2003 


\section{Inhaltsverzeichnis}

1 Einleitung 1

2 Atombeugung 4

2.1 Grundlagen . . . . . . . . . . . . . . . . . . 4

2.2 Atombeugung bei nicht-senkrechtem Einfall . . . . . . . . . . . 6

2.2.1 Atombeugung am Steg bei nicht-senkrechtem Einfall . . . . . 6

2.2.2 Atombeugung am Gitter bei nicht-senkrechtem Einfall . . . . 11

2.2.3 Das Beugungsbild des Atoms . . . . . . . . . . . . . . 15

2.3 Atombeugung bei senkrechtem Einfall . . . . . . . . . . . . . . . 22

2.3.1 Atombeugung am Gitter bei senkrechtem Einfall . . . . . . . . 22

2.3.2 Das Beugungsbild des Atoms . . . . . . . . . . . . 23

3 Trimerbeugung $\quad 25$

3.1 Das freie Trimer . . . . . . . . . . . . . . . . . . . . 25

3.1.1 Unterscheidbare Teilchen . . . . . . . . . . . . . . 26

3.1.2 Spinlose, ununterscheidbare Teilchen . . . . . . . . . . . . 30

3.1.3 Das Helium-Trimer ${ }^{4} \mathrm{He}_{3}$. . . . . . . . . . . . . . . . . . . . . . . . . . . 31

3.2 Die Streumatrix des Trimers . . . . . . . . . . . . . . . . . 33

3.2.1 Modellierung eines Molekularstrahlexperiments . . . . . . . . 34

3.2 .2 Das Streumatrixelement . . . . . . . . . . . . . . . 36

3.3 Die Übergangsamplitude des Trimers . . . . . . . . . . . . . . . . . . 39

3.3.1 Störungsentwicklung der Übergangsamplitude . . . . . . . . . 39

3.3.2 Die zweidimensionale Übergangsamplitude . . . . . . . . . . . 40

3.3.3 Trimerbeugung am Gitter bei nicht-senkrechtem Einfall . . . . 48

3.4 Das Beugungsbild des Trimers . . . . . . . . . . . . . . . . 59

3.4.1 Übertragung von Ergebnissen der Atombeugung . . . . . . . . 59

3.4.2 Die räumliche Ausdehnung der Bindungszustände . . . . . . . 62

3.4.3 Trimerbeugung am Gitter bei senkrechtem Einfall . . . . . . . 73

3.4.4 Physikalische Interpretation der effektiven Spaltbreite . . . . . 74

3.4.5 Senkrechter und nicht-senkrechter Einfall . . . . . . . . . . . 75

$\begin{array}{lll}4 & \text { Beugungsexperimente } & 77\end{array}$

4.1 Transmissionsmessung . . . . . . . . . . . . . . . . 79

4.2 Atom-Beugungsbilder . . . . . . . . . . . . . . . . 80

4.3 Trimer-Beugungsbilder . . . . . . . . . . . . . 86 
4.4 Dimer-Beugungsbilder . . . . . . . . . . . . . . . . . . . . . . . . . . . . . 89

4.4.1 Reduktion des Approximationsfehlers . . . . . . . . . . . 91

5 Inelastische Beugung und Spektroskopie $\quad 93$

5.1 Inelastische Dimerbeugung . . . . . . . . . . . . . . . . 94

5.1.1 Dimerbeugung am Gitter bei nicht-senkrechtem Einfall . . . . 94

5.1.2 Symmetrie und Auswahlregel der Dimerbeugung . . . . . . . . 95

5.2 Beugung von Wasserstoff-Dimeren . . . . . . . . . . . . . 97

5.2.1 Die freien Wasserstoff-Dimere $\left(\mathrm{o}-\mathrm{D}_{2}\right)_{2}$ und $\mathrm{p}-\mathrm{H}_{2}-\mathrm{O}-\mathrm{D}_{2} \ldots \ldots 9$

5.2.2 Beugungsbilder von $\left(\mathrm{o}-\mathrm{D}_{2}\right)_{2}$ und $\mathrm{p}-\mathrm{H}_{2}-\mathrm{o}-\mathrm{D}_{2} \ldots \ldots \ldots$

5.2.3 Experimentelle Realisierbarkeit inelastischer Beugung . . . . . 104

6 Zusammenfassung und Ausblick 105

$\begin{array}{ll}\text { A Attraktive Wechselwirkung } & 109\end{array}$

A.1 Die Transmissionsfunktion . . . . . . . . . . . . . . . . . . . 109

A.1.1 Die Phasenfunktion bei nicht-senkrechtem Einfall . . . . . . . 109

A.1.2 Die Phasenfunktion bei senkrechtem Einfall . . . . . . . . . . 112

A.1.3 Asymptotik der Kumulanten der Atombeugung . . . . . . . . 113

A.2 Korrekturen zur Geometrie der Gitterstege . . . . . . . . . . . 115

A.2.1 Abgerundete Stegkanten . . . . . . . . . . . . 115

A.2.2 Oberflächenrauhigkeit der Stegflanken . . . . . . . . . . 116

$\begin{array}{lr}\text { B Trimer-Bindungszustände } & 118\end{array}$

B.1 Spinlose, ununterscheidbare Teilchen . . . . . . . . . . . . . . 119

B.1.1 Die Faddeev-Gleichung . . . . . . . . . . . . . . . . . . . . . . 119

B.1.2 Unitäre Polapproximation . . . . . . . . . . . . . . . . 122

B.2 Das Helium-Trimer ${ }^{4} \mathrm{He}_{3} \ldots \ldots \ldots \ldots \ldots \ldots \ldots \ldots \ldots \ldots$

B.2.1 Die Faddeev-Gleichung für das Helium-Trimer . . . . . . . . . 124

B.2.2 Die Ortsraumdarstellung der Bindungszustände . . . . . . . 125

$\begin{array}{ll}\text { Literaturverzeichnis } & 129\end{array}$ 


\section{Kapitel 1}

\section{Einleitung}

Die Materie-Optik mit Atomen und Molekülen wurde in den vergangenen fünfzehn Jahren vom anschaulichen, qualitativen Lehrbuchversuch zu einem faszinierenden Präzisionsexperiment weiterentwickelt. Sie erlaubte beispielsweise erstmals den eindeutigen experimentellen Nachweis des zuvor lange umstrittenen zweiatomigen Helium-Moleküls ${ }^{4} \mathrm{He}_{2}$ [1, 2]. Eine moderne, auf der quantenmechanischen Mehrteilchen-Streutheorie basierende Beschreibung [3, 4] ermöglichte anschließend die genaue Auswertung des Experiments und die Bestimmung der extrem geringen Bindungsenergie dieses van der Waals-Dimers von nur $0,1 \mu \mathrm{eV}$ [5]. Da sich die Materie-Optik den Wellenaspekt von Teilchen zunutze macht, gehört auch die aktuelle Fragestellung nach quantenmechanischer Dekohärenz sehr massiver Teilchen mit vielen inneren Freiheitsgraden zu ihren Disziplinen, wie zum Beispiel beim Fulleren $\mathrm{C}_{70}[6]$.

Als der historische Ursprung der Materie-Optik kann de Broglies Hypothese aus dem Jahr 1923 angesehen werden, die einem Teilchen mit Impuls $p$ eine charakteristische Wellenlänge $\lambda=h / p$ zuordnet [7]. Bereits vier Jahre später untersuchten Davisson und Germer die Streuung eines Elektronenstrahls an einem Nickelkristall und beobachteten dabei eine gute Übereinkunft mit "Laue-Strahlen" und optischer Beugung, die sie mit der de Broglie-Wellenlänge der Elektronen plausibel machten [8]. Im Jahr 1930 beugten Estermann und Stern an einer LiF-Oberfläche Strahlen von Helium und Wasserstoff $\left(\mathrm{H}_{2}\right)$ [9]. Auch diese Experimente standen im Einklang mit de Broglies Entdeckung. Spätere Experimente an Kristallen betrafen beispielsweise die Neutronenbeugung und -interferometrie [10]. Daß Materie-Optik auch ohne Kristallgitter möglich ist, wies erstmals Boersch 1940 mit der Beugung eines Elektronenstrahls an einer Folienkante nach [11]. Er beobachtete dabei das aus der Lichtoptik bekannte Beugungsbild einer Halbebene.

Den Weg zur Materie-Optik mit größeren Teilchen bahnte die Herstellung mikrostrukturierter Spalte, die dem Problem begegnete, daß Atom- und Molekularstrahlen Kristalle nicht durchdringen können. Jönsson beobachtete 1961 kohärente Beugung eines Elektronenstrahls an bis zu fünf parallelen Spalten [12]. Leavitt und Bills demonstrierten 1969 die Beugung eines Kaliumstrahls an einem $23 \mu \mathrm{m}$ breiten Einzelspalt [13].

Die weitere Entwicklung wurde durch die Verfügbarkeit hoch genauer mikrofabri- 
zierter Transmissionsspalte und Transmissionsgitter aus den Materialien Gold oder Silizium-Nitrid beflügelt (z. B. Ref. [14]). So konnten Keith et al 1988 einen Strahl von Natrium-Atomen an einem ursprünglich für den Einsatz in der Röntgenoptik entwickelten Goldgitter beugen und die positive und negative zweite Beugungsordnung auflösen [15]. Dieselbe Gruppe realisierte vor acht Jahren erstmals ein AtomInterferometer mit drei Transmissionsgittern und einem $\mathrm{Na}_{2}$-Strahl [16]. Carnal und Mlynek demonstrierten 1989 das Youngsche Doppelspalt-Experiment mit metastabilen Helium-Atomen [17].

Gegenwärtig werden zwei unterschiedliche Zielsetzungen verfolgt. Die von Schöllkopf und Toennies [2] begonnene experimentelle Untersuchung der Beugung einzelner Atome und kleiner van der Waals-Cluster an einem Transmissionsgitter, die in verschiedenen Folgearbeiten weitergeführt und verbessert wurde, ist auf das quantitative Verständnis der dabei auftretenden Streuprozesse hin ausgerichtet [5, 18, 19]. Offensichtlich konnte das bisherige einfache Modell, bei dem die klassische Beugungstheorie mit der de Broglie-Wellenlänge kombiniert wird, diesen Anforderungen nicht gerecht werden. Die von Hegerfeldt und Köhler entwickelte quantenmechanische Beugungstheorie der Materie-Optik für Atome und schwach gebundene, zweiatomige Cluster (Dimere) [3, 4] berücksichtigt hingegen sowohl die schwache van der Waals-Wechselwirkung zwischen den Teilchen und dem Transmissionsgitter als auch die möglichen Streukanäle. Sie ermöglichte dadurch die korrekte Beschreibung der experimentellen Beobachtungen und Auswertung der Meßdaten. In dieser Hinsicht stellt ein MaterieOptikexperiment eine präzise Meßeinrichtung zur Bestimmung gewisser Eigenschaften der Atome und kleinen Cluster dar. Die andere Zielsetzung betrifft die bereits erwähnte Untersuchung der Kohärenz- und Dekohärenzeigenschaften schwerer Teilchen durch Arndt et al [6,20].

Besondere Aufmerksamkeit wird in der theoretischen Literatur dem dreiatomigen Molekül ${ }^{4} \mathrm{He}_{3}$ zuteil [21, 22, 23, 24, 25, 26, 27, 28, 29, 30, 31, 32, 33, 34, 35, 36, 37], das als Helium-Trimer bezeichnet wird. Dieser im Grundzustand laut Vorhersage mit nur circa $10 \mu \mathrm{eV}$ bereits relativ schwach gebundene van der Waals-Cluster ist der derzeit einzige bekannte Kandidat für das natürliche Auftreten eines weiteren, äußerst dicht unterhalb der Dissoziationsschwelle liegenden Bindungszustandes, der ein sogenannter Efimov-Zustand sein könnte. Efimov [38] zeigte 1970, daß in gewissen Dreiteilchensystemen die hypothetische Abschwächung der Paarwechselwirkungen die Einführung bis zu unendlich vieler Dreiteilchen-Bindungszustände zur Folge hätte. Im Umfeld der Kernphysik wurde daraufhin vergeblich nach einem Hinweis auf diesen Effekt gesucht. Der Nachweis des Efimov-Zustandes des Helium-Trimers sollte durch ein Beugungsexperiment und dessen quantitative theoretische Auswertung ermöglicht werden, wie die vorliegende Arbeit zeigt. In jüngster Zeit hat der Efimov-Effekt in einem anderen Zusammenhang erneutes Interesse erlangt, da er zum Beispiel die Bildung von ${ }^{85} \mathrm{Rb}$ Bose-Einstein-Kondensaten beeinflussen könnte [39].

Ein wesentliches Vorhaben dieser Arbeit ist es, die Beugungstheorie der MaterieOptik weiterzuentwickeln und auf schwach gebundene dreiatomige Cluster (Trimere) zu erweitern. Aufgrund der im Vergleich zum Dimer deutlich komplizierteren Struktur der Bindungszustände und der dadurch größeren Anzahl innerer Freiheitsgrade sind die Eigenschaften der Trimerbeugung nicht evident. Die Behandlung wird allge- 
mein geführt. Quantitative Aussagen werden, wo nötig, im Hinblick auf die Auswertung dreier umfangreicher Experimente von Brühl, Kalinin, Kornilov und Toennies immer am Beispiel des Helium-Trimers getroffen. Erste Meßreihen dieser Gruppe zur Trimerbeugung waren allerdings sehr großen, nicht reproduzierbaren Schwankungen unterworfen, die die gewünschte Bestimmung molekularer Eigenschaften des HeliumTrimers verhinderten. Um das Auflösungsvermögen zu erhöhen, wurden spätere Experimente unter einem vom Lot des Transmissionsgitters abweichenden Einfallswinkel durchgeführt. Dies verringert die (projizierte) Spaltbreite und erhöht dadurch die Empfindlichkeit, führt aber aufgrund der endlichen Tiefe des Gitters dazu, daß die Flanken der Gitterstege einseitig vom einfallenden Strahl „abgeschattet“ sind. Einige aus früheren Arbeiten bekannte Formeln der Materie-Optik besitzen für diesen Fall keine Gültigkeit mehr. Das 2. Kapitel befaßt sich daher zunächst mit der Formulierung der Atombeugung am Steg und am Transmissiongitter bei nicht-senkrechtem Einfall. Es beruht auf der allgemeinen Theorie der Atombeugung von Köhler [40] und stellt eine Vorarbeit für die Kapitel 3-5 dar. Kapitel 3 behandelt die Beugung von Trimeren. Entscheidend wird die Frage sein, ob und unter welchen Voraussetzungen der mittlere Paarabstand der drei Atome im Trimer, der beispielsweise im ${ }^{4} \mathrm{He}_{3}$ laut theoretischer Vorhersagen nur circa $10 \AA$ beträgt [32], analog zur Dimerbeugung eine Meßgröße darstellt und somit experimentell zugänglich ist. Darauf bauend werden im 4. Kapitel die Experimente von Brühl, Kalinin, Kornilov und Toennies ausgewertet. Außerdem werden die ebenfalls aufgenommenen Meßserien für das HeliumDimer ${ }^{4} \mathrm{He}_{2}$ ausgewertet und eine neue Methode zur Verbesserung der Genauigkeit der dabei erhaltenen Ergebnisse vorgeschlagen.

In Kapitel 5 wird die Perspektive gewechselt, um den bislang bei der Clusterbeugung experimentell nicht genutzten Aspekt der inelastischen Beugung zu untersuchen. Beispielsweise wäre es damit prinzipiell möglich, einen Filter für Cluster vorgegebener innerer Zustände zu realisieren sowie Spektroskopie von Clustern zu betreiben. Im Unterschied zu in der Molekülspektroskopie gängigen Verfahren, wie etwa der Infrarot-Absorptionsspektroskopie [41], besteht der Anregungsmechanismus hier im „Stoß“ mit dem Transmissionsgitter anstelle von elektromagnetischer Ankopplung und stellt somit eine echte Alternative dar. Fraglich ist zunächst, ob sich gegenwärtige Molekularstrahlapparaturen für Versuche zur inelastischen Beugung eignen. Am Beispiel der Wasserstoff-Dimere $\left(\mathrm{D}_{2}\right)_{2}$ und $\mathrm{H}_{2} \mathrm{D}_{2}$ wird die Realisierbarkeit eines konkreten Experiments untersucht.

Die Kapitel 2-5 werden ergänzt durch zwei Anhänge. Anhang A befaßt sich mit der attraktiven Wechselwirkung zwischen den Atomen und dem Transmissionsgitter und zeigt unter anderem, daß der Einfluß der Wechselwirkung einer einfachen asymptotischen Gesetzmäßigkeit folgt. In Anhang B werden die Faddeev-Gleichungen für das freie Trimer vorgestellt, um zunächst einige benötigte Eigenschaften von TrimerZuständen herzuleiten, die in Kapitel 3 keinen Platz gefunden haben. Anschließend wird, basierend auf den Faddeev-Gleichungen, ein Verfahren zur numerischen Berechnung von Trimer-Bindungswellenfunktionen erläutert. Die Wellenfunktionen werden bei der quantitativen Analyse einiger Rechnungsschritte von Kapitel 3 benötigt. 


\section{Kapitel 2}

\section{Atombeugung}

Die Theorie der Atombeugung stellt den Ausgangspunkt für das Verständnis der Beugung größerer Cluster dar. Von physikalischem Interesse ist dabei vor allem der Unterschied zur optischen Beugung, der durch die schwache Oberflächenwechselwirkung der Atome mit dem Beugungsobjekt entsteht. Dieser Effekt äußert sich im Beugungsbild einer Spaltblende oder eines Transmissionsgitters in Abhängigkeit von der Geschwindigkeit des Atomstrahls, gleichsam als wäre die Spaltbreite etwas verringert [18]. Die Spaltbreite ist eine Meßgröße. Wünschenswert ist eine kleine Spaltbreite, da die relative Änderung dort stärker ins Gewicht fällt und somit eine bessere Empfindlichkeit bei der Auswertung erlaubt. Eine Möglichkeit dazu besteht in der Drehung des Transmissionsgitters aus dem Lot gegenüber dem einfallenden Teilchenstrahl: die projizierte Spaltbreite verringert sich mit dem Kosinus des Drehwinkels. Die Verbesserung der Auflösung wird sich besonders für die Untersuchung des Helium-Dimers und -Trimers in Kapitel 4 von Vorteil erweisen. Eine quantitative Abschätzung der Verbesserung muß allerdings auf Abschnitt 3.4.5 verschoben werden, da erst dort die notwendigen Formeln zur Verfügung stehen.

In diesem Kapitel werden die Gleichungen der Atombeugung bei gegenüber der senkrechten Ausrichtung um den Winkel $\theta^{\prime}$ verdrehtem Transmissionsgitter mit dadurch einseitig abgeschatteten Stegflanken abgeleitet. Wegen des speziellen Profils der in den Experimenten verwendeten Gitter tritt diese Abschattung aber erst oberhalb eines Winkels $\theta^{\prime}=\beta$ auf; zur Vereinfachung der Nomenklatur wird hier und in den folgenden Kapiteln die Bezeichnung „nicht-senkrechter Einfall“ für den Fall $\theta^{\prime}>\beta$ reserviert. (Der Fall $0<\theta^{\prime}<\beta$ läßt sich mit dem bereits bekannten Formalismus behandeln.) Die Ergebnisse werden als Bausteine für die anschließenden Kapitel dienen.

\subsection{Grundlagen}

Zunächst werden für die Atombeugung einige Grundlagen der Theorie der Potentialstreuung resümiert (für eine Einführung siehe z. B. Ref. [42]). Es wird in allen Fällen angenommen, daß die kinetische Energie der Atome wesentlich geringer ist als ihre typischen elektronischen Anregungsenergien. Daher werden Atome im folgenden als Punktteilchen ohne innere Freiheitsgrade betrachtet. Die Wechselwirkung mit dem 
Beugungsobjekt wird durch ein Potential $W$ modelliert. Für ein Atom der Masse $m$ mit der Ortskoordinate $\mathbf{x}$ lautet der vollständige Hamilton-Operator demzufolge

$$
H=H_{0}+W \quad \text { mit } \quad H_{0}=\frac{\hat{\mathbf{p}}^{2}}{2 m} .
$$

Die gemeinsamen stationären, uneigentlichen Eigenzustände des freien HamiltonOperators $H_{0}$ zur Energie $E^{\prime}=\left|\mathbf{p}^{\prime}\right|^{2} / 2 m$ und des Impulsoperators zum Impuls $\mathbf{p}^{\prime}$ werden mit $\left|\mathbf{p}^{\prime}\right\rangle$ bezeichnet. Die stationären, uneigentlichen Streuzustände des vollständigen Hamilton-Operators $H$ zur Energie $E^{\prime}$ werden mit $\left|\mathbf{p}^{\prime}, \pm\right\rangle$ bezeichnet. Sie erfüllen die Lippmann-Schwinger-Gleichung

$$
\left|\mathbf{p}^{\prime}, \pm\right\rangle=\left|\mathbf{p}^{\prime}\right\rangle+G_{0}\left(E^{\prime} \pm \mathrm{i} 0\right) W\left|\mathbf{p}^{\prime}, \pm\right\rangle .
$$

Die freie Resolvente ist darin definiert durch

$$
G_{0}(z):=\left[z-H_{0}\right]^{-1} .
$$

Ein im Experiment durch eine Quelle präpariertes Atom wird durch seine Impulsverteilung $\psi\left(\mathbf{p}^{\prime}\right)$ charakterisiert. In der stationären Streutheorie läßt sich die Wahrscheinlichkeitsdichte dafür, daß das Atom durch die Wechselwirkung mit dem Potential $W$ in den auslaufenden Impuls $\mathbf{p}$ mit Energie $E=|\mathbf{p}|^{2} / 2 m$ gestreut wird, durch die zum Hamilton-Operator (2.1) gehörende Streumatrix $S$ ausdrücken.

$$
w(\mathbf{p} ; \psi):=\left|\int \mathrm{d}^{3} p^{\prime} \psi\left(\mathbf{p}^{\prime}\right)\left\langle\mathbf{p}|S| \mathbf{p}^{\prime}\right\rangle\right|^{2}
$$

Für das Element der Streumatrix zwischen uneigentlichen Zuständen existiert die Zerlegung

$$
\left\langle\mathbf{p}|S| \mathbf{p}^{\prime}\right\rangle=\delta^{(3)}\left(\mathbf{p}-\mathbf{p}^{\prime}\right)-2 \pi \mathrm{i} \delta\left(E-E^{\prime}\right) t^{\text {at }}\left(\mathbf{p} ; \mathbf{p}^{\prime}\right),
$$

wobei die atomare Übergangsamplitude $t^{\text {at }}\left(\mathbf{p} ; \mathbf{p}^{\prime}\right)$ zwischen den Impulsen $\mathbf{p}^{\prime}$ und $\mathbf{p} \equiv$ $\mathbf{p}^{\prime}+\Delta \mathbf{p}$ die explizite Form

$$
t^{\text {at }}\left(\mathbf{p} ; \mathbf{p}^{\prime}\right):=\left\langle\mathbf{p}|W| \mathbf{p}^{\prime},+\right\rangle
$$

besitzt.

In Experimenten zur Materie-Beugung kommen üblicherweise Spaltblenden oder Transmissionsgitter als Beugungsobjekte zum Einsatz, die entlang der Spalte als translationsinvariant angenommen werden dürfen. Das Potential $W(\mathbf{x})$ hängt in dieser Idealisierung nur von zwei Koordinaten ab. Das Koordinatensystem wird daher so gewählt, daß die $x_{3}$-Achse parallel zu den Spalten des im Ursprung liegenden Beugungsobjektes verläuft. Aus Symmetriegründen findet in dieser Richtung keine Streuung statt, und der Streuzustand $\left|\mathbf{p}^{\prime},+\right\rangle$ faktorisiert in den Produktzustand

$$
\left|\mathbf{p}^{\prime},+\right\rangle=\left|p_{1}^{\prime}, p_{2}^{\prime},+\right\rangle\left|p_{3}^{\prime}\right\rangle .
$$

In der Übergangsamplitude (2.6) kann deswegen eine Deltafunktion ausfaktorisiert werden, die die Erhaltung der Impulskomponente $p_{3}^{\prime}$ widerspiegelt. Das Streuproblem 
ist dadurch formal auf zwei Dimensionen reduziert. Es ist sinnvoll, die Notation diesem Umstand anzupassen. Mit dem kursiv gesetzten Vektorsymbol $\boldsymbol{p}^{\prime}$ wird im folgenden die zweidimensionale Projektion des Vektors $\mathbf{p}^{\prime}$ in die $\left(x_{1}, x_{2}\right)$-Ebene bezeichnet. Entsprechend wird die Projektion des auslaufenden Impulses mit $\boldsymbol{p}$ bezeichnet. Aus Gleichung (2.6) folgt also

$$
t^{\text {at }}\left(\mathbf{p} ; \mathbf{p}^{\prime}\right)=\delta\left(\Delta p_{3}\right) t^{\text {at( }(2)}\left(\boldsymbol{p} ; \boldsymbol{p}^{\prime}\right),
$$

wobei

$$
t^{\mathrm{at}(2)}\left(\boldsymbol{p} ; \boldsymbol{p}^{\prime}\right):=\left\langle\boldsymbol{p}|W| \boldsymbol{p}^{\prime},+\right\rangle
$$

die zweidimensionale Übergangsamplitude ist.

Im weiteren wird immer vorausgesetzt, daß die von Hegerfeldt und Köhler [3] formulierten „Beugungsbedingungen“ erfüllt sind. Diese fordern insbesondere, daß die de Broglie-Wellenlänge der Atome im Teilchenstrahl wesentlich kleiner ist als eine typische Längenskala $s$ des Beugungsobjektes. In zwei Dimensionen muß also gelten

$$
\lambda_{\mathrm{dB}}=\frac{2 \pi \hbar}{\left|\boldsymbol{p}^{\prime}\right|} \ll s .
$$

Für ein Gitter mit der Spaltbreite $s_{0}$, Periode $d$ und Stegbreite $a=d-s_{0}$ bedeutet dies $\lambda_{\mathrm{dB}} \ll s_{0}$ und $\lambda_{\mathrm{dB}} \ll a$.

\section{$2.2 \quad$ Atombeugung bei nicht-senkrechtem Einfall}

Die in früheren Arbeiten zur Atom- und Dimerbeugung im Hinblick auf die damaligen Experimente besonders genau untersuchte Situation des senkrechten Einfalls $[5,18,40]$ ist wegen der erwähnten einseitigen Abschattung der Stegflanken der in den Experimenten von Brühl, Kalinin, Kornilov und Toennies verwendeten Transmissionsgitter nicht unmittelbar auf die Situation des nicht-senkrechten Einfalls übertragbar. Daher ist ein Rückgriff auf die Grundlagen der von Köhler [40] vorgestellten Theorie der Atombeugung nötig. Parallel zum dortigen Vorgehen wird zunächst nur die Beugung am rein repulsiven Einzelsteg behandelt. Der repulsive Anteil $W_{\text {rep }}$ des Potentials $W$ wird durch ideal-reflektierende Randbedingungen auf der Oberfläche des Stegs modelliert. Die schwache attraktive Oberflächenwechselwirkung wird danach störungstheoretisch hinzugefügt. Anschließend werden mehrere Stege zu einem Gitter kombiniert.

\subsubsection{Atombeugung am Steg bei nicht-senkrechtem Einfall}

Die Ortsraumdarstellung des atomaren Streuzustands (2.7) bezüglich der relevanten Koordinaten in der $\left(x_{1}, x_{2}\right)$-Ebene wird mit

$$
\psi\left(\boldsymbol{k}^{\prime}, \boldsymbol{x}\right):=2 \pi \hbar\left\langle\boldsymbol{x} \mid \boldsymbol{p}^{\prime},+\right\rangle
$$

bezeichnet, wobei $\boldsymbol{k}^{\prime}:=\boldsymbol{p}^{\prime} / \hbar$ gesetzt wurde. Der freie einlaufende Zustand lautet

$$
\psi_{\text {inc }}\left(\boldsymbol{k}^{\prime}, \boldsymbol{x}\right):=2 \pi \hbar\left\langle\boldsymbol{x} \mid \boldsymbol{p}^{\prime}\right\rangle=\exp \left(\mathrm{i} \boldsymbol{k}^{\prime} \cdot \boldsymbol{x}\right) .
$$




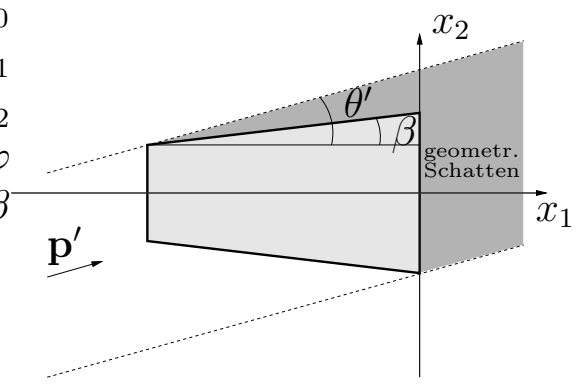

Abbildung 2.1: Idealisierte Keilstumpfform eines Gittersteges der in Experimenten von Brühl, Kalinin, Kornilov und Toennies verwendeten Transmissionsgitter. Überschreitet der Einfallswinkel $\theta^{\prime}$ den Keilwinkel $\beta$, so wird eine Flanke des Steges abgeschattet.

Die Lippmann-Schwinger-Gleichung (2.2) stellt formal eine Zerlegung des gesamten Streuzustands dar:

$$
\psi=\psi_{\text {inc }}+\psi_{\text {scatt }} .
$$

Darin kann der Term

$$
\psi_{\text {scatt }}\left(\boldsymbol{k}^{\prime}, \boldsymbol{x}\right):=2 \pi \hbar\left\langle\boldsymbol{x}\left|G_{0}^{(2)}\left(E^{\prime(2)}+\mathrm{i} 0\right) W_{\text {rep }}\right| \boldsymbol{p}^{\prime},+\right\rangle
$$

als „gestreuter Anteil“ des Streuzustands zur Energie $E^{\prime(2)}:=\hbar^{2}\left|\boldsymbol{k}^{\prime}\right|^{2} / 2 m$ interpretiert werden. Die freie Resolvente in zwei Dimensionen hat die Form

$$
G_{0}^{(2)}(z)=\left[z-\hat{\boldsymbol{p}}^{2} / 2 m\right]^{-1} .
$$

Für Orte $\boldsymbol{x}$ außerhalb des Steges erfüllt der Streuzustand $\psi\left(\boldsymbol{k}^{\prime}, \boldsymbol{x}\right)$ die zweidimensionale Helmholtz-Gleichung $\left(\Delta_{\boldsymbol{x}}+\left|\boldsymbol{k}^{\prime}\right|^{2}\right) \psi=0$. Die Ortsraumdarstellung der freien Resolvente

$$
G_{0}^{(2)}\left(\left|\boldsymbol{k}^{\prime}\right| ; \boldsymbol{x}, \boldsymbol{x}^{\prime}\right):=-\frac{\hbar^{2}}{2 m}\left\langle\boldsymbol{x}\left|G_{0}^{(2)}\left(E^{\prime(2)}+\mathrm{i} 0\right)\right| \boldsymbol{x}^{\prime}\right\rangle
$$

ist eine Fundamentallösung der Differentialgleichung

$$
\left(\Delta_{\boldsymbol{x}}+\left|\boldsymbol{k}^{\prime}\right|^{2}\right) G_{0}^{(2)}\left(\left|\boldsymbol{k}^{\prime}\right| ; \boldsymbol{x}, \boldsymbol{x}^{\prime}\right)=-\delta^{(2)}\left(\boldsymbol{x}-\boldsymbol{x}^{\prime}\right),
$$

die sich asymptotisch für große $\left|\boldsymbol{x}-\boldsymbol{x}^{\prime}\right|$ wie eine auslaufende Kreiswelle verhält. Sie ist proportional zur Hankelfunktion $H_{0}^{(1)}$ [43, Kap. 3.10].

In großen Entfernungen $|\boldsymbol{x}|$ vom Beugungsobjekt kann der Streuanteil $\psi_{\text {scatt }}$ in einem weiten Winkelbereich um die Vorwärtsrichtung herum durch einen „Beugungsanteil" $\psi_{\text {diffr }}$ approximiert werden [40]. Für die Zwecke der vorliegenden Arbeit wird dieser Beugungsanteil in der Form

$$
\begin{aligned}
\psi_{\text {diffr }}\left(\boldsymbol{k}^{\prime}, \boldsymbol{x}\right):=\int_{\mathcal{A}} \mathrm{d} S\left(\boldsymbol{x}^{\prime}\right)\left[G_{0}^{(2)}\left(\left|\boldsymbol{k}^{\prime}\right| ; \boldsymbol{x}, \boldsymbol{x}^{\prime}\right) \frac{\partial}{\partial n\left(\boldsymbol{x}^{\prime}\right)} \psi_{\mathrm{inc}}\left(\boldsymbol{k}^{\prime}, \boldsymbol{x}^{\prime}\right)\right. \\
\left.\quad-\psi_{\mathrm{inc}}\left(\boldsymbol{k}^{\prime}, \boldsymbol{x}^{\prime}\right) \frac{\partial}{\partial n\left(\boldsymbol{x}^{\prime}\right)} G_{0}^{(2)}\left(\left|\boldsymbol{k}^{\prime}\right| ; \boldsymbol{x}, \boldsymbol{x}^{\prime}\right)\right]
\end{aligned}
$$

geschrieben, wobei sich das Linienintegral über die in Abbildung 2.2 eingezeichnete "geometrische Schattenlinie“ $\mathcal{A}$ erstreckt und $\boldsymbol{n}\left(\boldsymbol{x}^{\prime}\right)$ die Kurvennormale bezeichnet. 


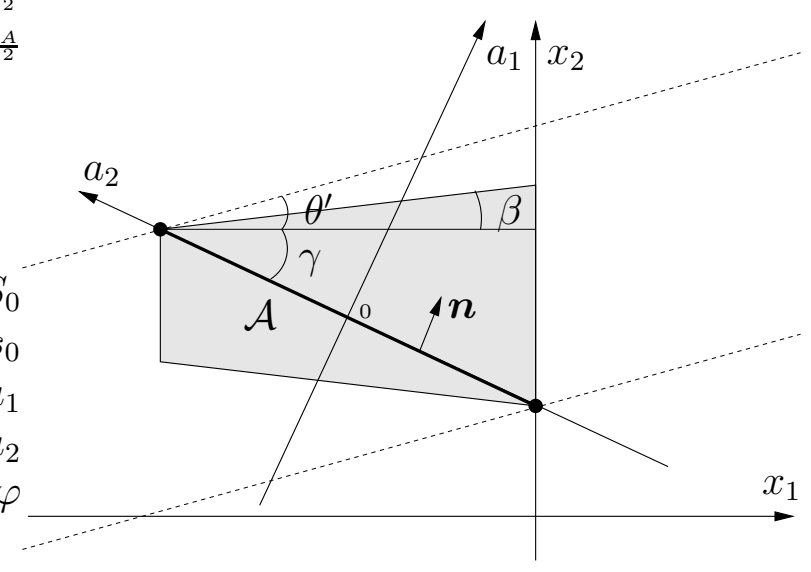

Abbildung 2.2: Beugung am Steg bei nicht-senkrechtem Einfall. Das Koordinatensystem $\left(a_{1}, a_{2}\right)$ ist so gewählt, daß die geometrische Schattenlinie $\mathcal{A}$ (dick gezeichnet) mit der $a_{2}$-Achse zusammenfällt und ihre Mitte im Ursprung liegt. Die Länge der geometrischen Schattenlinie ist $A$.

Ausgedrückt im Koordinatensystem $\left(a_{1}, a_{2}\right)$, das so gewählt ist, daß die Achse $a_{2}$ mit der geometrischen Schattenlinie zusammenfällt und der Ursprung in der Mitte von $\mathcal{A}$ liegt (vgl. Abb. 2.2), lautet Gleichung (2.15)

$$
\psi_{\mathrm{diffr}}\left(\boldsymbol{k}^{\prime}, \boldsymbol{x}\right)=\left.\int_{-\frac{A}{2}}^{\frac{A}{2}} \mathrm{~d} a_{2}^{\prime} e^{\mathrm{i} k_{a_{2}}^{\prime} a_{2}^{\prime}}\left[\mathrm{i} k_{a_{1}}^{\prime}-\frac{\partial}{\partial a_{1}^{\prime}}\right] G_{0}^{(2)}\left(\left|\boldsymbol{k}^{\prime}\right| ; \boldsymbol{x}, \boldsymbol{x}^{\prime}\right)\right|_{a_{1}^{\prime}=0} .
$$

Darin bedeuten $k_{a_{1}}^{\prime}$ und $k_{a_{2}}^{\prime}$ die Projektionen des einlaufenden Wellenvektors $\boldsymbol{k}^{\prime}$ auf die Achsen $a_{1}$ beziehungsweise $a_{2}$. Die Länge der geometrischen Schattenlinie ist $A$. Mit der Definition des auslaufenden Wellenvektors $\boldsymbol{k}:=\left|\boldsymbol{k}^{\prime}\right| \boldsymbol{x} /|\boldsymbol{x}|$, und somit $\left|\boldsymbol{k}^{\prime}\right|=|\boldsymbol{k}|$, gilt die asymptotische Entwicklung [44]

$$
G_{0}^{(2)}\left(|\boldsymbol{k}| ; \boldsymbol{x}, \boldsymbol{x}^{\prime}\right)=\frac{\mathrm{i}}{4} H_{0}^{(1)}\left(|\boldsymbol{k}|\left|\boldsymbol{x}-\boldsymbol{x}^{\prime}\right|\right) \stackrel{|\boldsymbol{x}| \rightarrow \infty}{\sim} \frac{\mathrm{i}}{4} \sqrt{\frac{2}{\pi|\boldsymbol{k}|}} \mathrm{e}^{-\mathrm{i} \boldsymbol{k} \cdot \boldsymbol{x}^{\prime}} \frac{\mathrm{e}^{\mathrm{i}(|\boldsymbol{k}||\boldsymbol{x}|-\pi / 4)}}{\sqrt{|\boldsymbol{x}|}} .
$$

Aus Gleichung (2.16) folgt also für große Abstände vom Steg

$$
\psi_{\text {diffr }}\left(\boldsymbol{k}^{\prime}, \boldsymbol{x}\right) \stackrel{|\boldsymbol{x}| \rightarrow \infty}{\sim}-\frac{k_{a_{1}}+k_{a_{1}}^{\prime}}{2|\boldsymbol{k}|} \frac{1}{\sqrt{\lambda_{\mathrm{dB}}}} \frac{\mathrm{e}^{\mathrm{i}(|\boldsymbol{k}||\boldsymbol{x}|-\pi / 4)}}{\sqrt{|\boldsymbol{x}|}} \int_{-\frac{A}{2}}^{\frac{A}{2}} \mathrm{~d} a_{2}^{\prime} \mathrm{e}^{-\mathrm{i} \Delta k_{a_{2}} a_{2}^{\prime}},
$$

wobei der Impulsübertrag $\Delta k_{a_{2}}:=k_{a_{2}}-k_{a_{2}}^{\prime}$ eingeführt wurde. Die Integration von Gleichung (2.17) liefert

$$
\psi_{\text {diffr }}\left(\boldsymbol{k}^{\prime}, \boldsymbol{x}\right) \stackrel{|\boldsymbol{x}| \rightarrow \infty}{\sim}-\frac{k_{a_{1}}+k_{a_{1}}^{\prime}}{2|\boldsymbol{k}|} \frac{1}{\sqrt{\lambda_{\mathrm{dB}}}} \frac{\mathrm{e}^{\mathrm{i}(|\boldsymbol{k}||\boldsymbol{x}|-\pi / 4)}}{\sqrt{|\boldsymbol{x}|}} \frac{\sin \left(\Delta k_{a_{2}} A / 2\right)}{\Delta k_{a_{2}} / 2} .
$$

Da aber der gestreute Anteil $\psi_{\text {scatt }}$ nach den Gleichungen (2.13), (2.14) und (2.9) über

$$
\psi_{\text {scatt }}\left(\boldsymbol{k}^{\prime}, \boldsymbol{x}\right)=-\mathrm{i} \frac{\pi m}{\hbar} \int \mathrm{d}^{2} x^{\prime} H_{0}^{(1)}\left(\left|\boldsymbol{k} \| \boldsymbol{x}-\boldsymbol{x}^{\prime}\right|\right)\left\langle\boldsymbol{x}^{\prime}\left|W_{\text {rep }}\right| \boldsymbol{p}^{\prime},+\right\rangle
$$




$$
\underset{|\boldsymbol{x}| \rightarrow \infty}{\sim}-\mathrm{i} 2 \pi^{2} m \sqrt{\frac{2}{\pi|\boldsymbol{k}|}} \frac{\mathrm{e}^{\mathrm{i}(|\boldsymbol{k}||\boldsymbol{x}|-\pi / 4)}}{\sqrt{|\boldsymbol{x}|}} t_{\mathrm{rep}}^{\mathrm{at}(2)}\left(\boldsymbol{p} ; \boldsymbol{p}^{\prime}\right)
$$

mit der Übergangsamplitude des repulsiven Steges zusammenhängt, wobei $\boldsymbol{p}=\hbar \boldsymbol{k}$ identifiziert wurde, folgt durch Vergleich des asymptotischen Verhaltens von $\psi_{\text {diffr }}$ und $\psi_{\text {scatt }}$ im Bereich des Beugungsbildes

$$
t_{\mathrm{rep}}^{\mathrm{at}(2)}\left(\boldsymbol{p} ; \boldsymbol{p}^{\prime}\right)=-\frac{\mathrm{i}}{2} \frac{p_{a_{1}}+p_{a_{1}}^{\prime}}{(2 \pi)^{2} m \hbar} \frac{\sin \left(\Delta p_{a_{2}} A / 2 \hbar\right)}{\Delta p_{a_{2}} / 2 \hbar} .
$$

Diese Übergangsamplitude hat ein Maximum der Breite $\Delta p_{a_{2}}=4 \pi \hbar / A$ um die Vorwärtsrichtung, das in den Experimenten von Brühl, Kalinin, Kornilov und Toennies typischerweise Beugungswinkeln von einigen Milliradian entspricht. Die Atome werden also im wesentlichen in kleine Winkel um die Vorwärtsrichtung herum gebeugt.

\section{Einbezug der attraktiven Wechselwirkung}

Aus früheren Arbeiten ist bekannt, daß die attraktive van der Waals-Wechselwirkung zwischen den Atomen im Teilchenstrahl und dem Steg für die quantitative Beschreibung von Experimenten nicht vernachlässigt werden darf. Ist diese Wechselwirkung, die durch ein Potential $W_{\text {att }}$ modelliert wird, ausreichend schwach gegenüber der kinetischen Energie der Teilchen, so ändert sie die Übergangsamplitude (2.18) qualitativ nicht wesentlich und kann störungstheoretisch behandelt werden. Eine ausführlichere Diskussion findet sich in Ref. [40]. Es wird angenommen, daß die Reichweite von $W_{\text {att }}$ nur wenige Nanometer von der Stegoberfläche beträgt. Die Übergangsamplitude des Steges wird in der sogenannten Distorted-Wave-Born-Approximation [45, Kap. 9.1.3] in der Form

$$
t^{\text {at(2) }}\left(\boldsymbol{p} ; \boldsymbol{p}^{\prime}\right) \simeq t_{\mathrm{rep}}^{\mathrm{at}(2)}\left(\boldsymbol{p} ; \boldsymbol{p}^{\prime}\right)+K\left(\boldsymbol{p} ; \boldsymbol{p}^{\prime}\right)
$$

mit dem Korrekturterm [40]

$$
K\left(\boldsymbol{p} ; \boldsymbol{p}^{\prime}\right):=\frac{1}{(2 \pi \hbar)^{2}} \int \mathrm{d}^{2} x \mathrm{e}^{-\mathrm{i} \Delta \boldsymbol{p} \cdot \boldsymbol{x} / \hbar} W_{\text {att }}(\boldsymbol{x}),
$$

geschrieben, wobei der Integrationsbereich in (2.20) so einzuschränken ist, daß $\boldsymbol{x}$ sich nicht im durch den Gittersteg „blockierten“ Strahlbereich, speziell also nicht zwischen den beiden gestrichelten Linien in Abbildung 2.2 befindet. Es ist zweckmäßig, ein weiteres Koordinatensystem $\left(x_{\|}, x_{\perp}\right)$ einzuführen, daß der Beugung in kleine Winkel um die Vorwärtsrichtung herum angepaßt ist: die $x_{\|}$-Achse verlaufe parallel zum einlaufenden Impuls $\boldsymbol{p}^{\prime}$, die $x_{\perp}$-Achse senkrecht dazu, so daß wieder ein Rechtssystem entsteht. Sein Ursprung falle mit demjenigen von $\left(a_{1}, a_{2}\right)$ zusammen. Mit dem in Abbildung 2.2 eingezeichneten geometrischen Winkel $\gamma$ und dem Einfallswinkel $\theta^{\prime}$ lautet die Transformation zwischen den beiden Koordinatensystemen $\left(a_{1}, a_{2}\right)$ und $\left(x_{\|}, x_{\perp}\right)$ für Vektorkomponenten

$$
\left(\begin{array}{l}
a_{1} \\
a_{2}
\end{array}\right)_{a}=\left(\begin{array}{cc}
\sin \left(\gamma+\theta^{\prime}\right) & \cos \left(\gamma+\theta^{\prime}\right) \\
-\cos \left(\gamma+\theta^{\prime}\right) & \sin \left(\gamma+\theta^{\prime}\right)
\end{array}\right)\left(\begin{array}{c}
x_{\|} \\
x_{\perp}
\end{array}\right)_{\|} .
$$


Der Korrekturterm läßt sich nun schreiben als

$$
K\left(\boldsymbol{p} ; \boldsymbol{p}^{\prime}\right)=\frac{1}{(2 \pi \hbar)^{2}} \int_{\mathbb{R} \backslash \widetilde{\mathcal{A}}} \mathrm{d} x_{\perp} \mathrm{e}^{-\mathrm{i} \Delta p_{\perp} x_{\perp} / \hbar} \int_{\mathbb{R}} \mathrm{d} x_{\|} \mathrm{e}^{-\mathrm{i} \Delta p_{\|} x_{\|} / \hbar} W_{\text {att }}(\boldsymbol{x}),
$$

wobei die geforderte Einschränkung des Integrationsbereichs durch die Ausnahme des Intervalls $\widetilde{\mathcal{A}}:=\left[-A \sin \left(\gamma+\theta^{\prime}\right) / 2, A \sin \left(\gamma+\theta^{\prime}\right) / 2\right]$ im Integral über $x_{\perp}$ berücksichtigt wird. Wie eine kurze Abschätzung zeigt, ist der Exponent $\Delta p_{\|} x_{\|} / \hbar$ vernachlässigbar klein: Wegen $|\boldsymbol{p}|=\left|\boldsymbol{p}^{\prime}\right|$ und, der Definition des angepaßten Koordinatensystems zufolge, $p_{\|}^{\prime}=\left|\boldsymbol{p}^{\prime}\right|$ und $p_{\perp}^{\prime}=0$ gilt

$$
\Delta p_{\|} x_{\|} / \hbar=\left(\sqrt{\left|\boldsymbol{p}^{\prime}\right|^{2}-p_{\perp}^{2}}-\left|\boldsymbol{p}^{\prime}\right|\right) x_{\|} / \hbar \approx-\frac{p_{\perp}^{2} x_{\|}}{2\left|\boldsymbol{p}^{\prime}\right| \hbar} .
$$

Aufgrund der kurzen Reichweite der attraktiven Wechselwirkung $W_{\text {att }}$ ist im Integranden aber $x_{\|} \lesssim A$ erfüllt, so daß im Bereich $p_{\perp} \approx 4 \pi \hbar / A \sin \left(\gamma+\theta^{\prime}\right)$ des Maximums der Übergangsamplitude des repulsiven Steges (2.18) bei typischen Werten der de Broglie-Wellenlänge $\lambda_{\mathrm{dB}}=1 \AA$ und dem charakteristischen Winkel $\gamma=20^{\circ}$ folgt

$$
\Delta p_{\|} x_{\|} / \hbar \approx \frac{4 \pi \lambda_{\mathrm{dB}}}{A \sin ^{2}\left(\gamma+\theta^{\prime}\right)} \approx 3 \times 10^{-2} \ll 2 \pi .
$$

Mit der gleichen Argumentation darf aber im Integral (2.22) der Exponent $\Delta p_{\perp} x_{\perp} / \hbar$ näherungsweise um den Term $-\cot \left(\gamma+\theta^{\prime}\right) \Delta p_{\|} x_{\perp} / \hbar$ ergänzt werden, so daß der Korrekturterm nach Substitution von $x_{\perp}=: a_{2} \sin \left(\gamma+\theta^{\prime}\right)$ lautet

$$
\left.K\left(\boldsymbol{p} ; \boldsymbol{p}^{\prime}\right) \simeq \frac{\sin \left(\gamma+\theta^{\prime}\right)}{(2 \pi \hbar)^{2}} \int_{\mathbb{R} \backslash \mathcal{A}} \mathrm{d} a_{2} \mathrm{e}^{-\mathrm{i} \Delta p_{a_{2}} a_{2} / \hbar} \int_{\mathbb{R}} \mathrm{d} x_{\|} W_{\mathrm{att}}(\boldsymbol{x})\right|_{x_{\perp}=a_{2} \sin \left(\gamma+\theta^{\prime}\right)} .
$$

Im ersten Integral ist nun die geometrische Schattenlinie $\mathcal{A}=[-A / 2, A / 2]$ aus dem Integrationsbereich ausgenommen. Um den Korrekturterm mit der Übergangsamplitude des repulsiven Steges (2.18) zusammenfassen zu können, wird er durch Erweitern approximativ auf die Form

$$
K\left(\boldsymbol{p} ; \boldsymbol{p}^{\prime}\right) \simeq-\frac{\mathrm{i}}{2} \frac{p_{a_{1}}+p_{a_{1}}^{\prime}}{(2 \pi)^{2} m \hbar} \int_{\mathbb{R} \backslash \mathcal{A}} \mathrm{d} a_{2} \mathrm{e}^{-\mathrm{i} \Delta p_{a_{2}} a_{2} / \hbar}\left[1-\mathrm{e}^{\mathrm{i} \phi_{a}\left(\boldsymbol{p}^{\prime} ; a_{2}\right)}\right]
$$

mit der Phasenfunktion

$$
\phi_{a}\left(\boldsymbol{p}^{\prime} ; a_{2}\right):=-\left.\frac{m}{\left|\boldsymbol{p}^{\prime}\right| \hbar} \int \mathrm{d} x_{\|} W_{\mathrm{att}}(\boldsymbol{x})\right|_{x_{\perp}=a_{2} \sin \left(\gamma+\theta^{\prime}\right)} \quad \text { für } \quad a_{2} \in \mathbb{R} \backslash \mathcal{A}
$$

gebracht, wobei im Nenner von (2.25)

$$
\frac{p_{a_{1}}+p_{a_{1}}^{\prime}}{2 \sin \left(\gamma+\theta^{\prime}\right)} \approx p_{\|}^{\prime}=\left|\boldsymbol{p}^{\prime}\right|
$$

ersetzt wurde. Diese Berücksichtigung der attraktiven Wechselwirkung entspricht der Eikonalapproximation [40, 46]. Da der repulsive Anteil der Übergangsamplitude (2.18) als

$$
t_{\text {rep }}^{\text {at }(2)}\left(\boldsymbol{p} ; \boldsymbol{p}^{\prime}\right)=-\frac{\mathrm{i}}{2} \frac{p_{a_{1}}+p_{a_{1}}^{\prime}}{(2 \pi)^{2} m \hbar} \int_{\mathcal{A}} \mathrm{d} a_{2} \mathrm{e}^{-\mathrm{i} \Delta p_{a_{2}} a_{2} / \hbar}
$$


geschrieben werden kann, lassen sich die Terme (2.24) und (2.26) entsprechend Gleichung (2.19) nun zusammenfassen. Es folgt also für die Übergangsamplitude am Steg bei nicht-senkrechtem Einfall

$$
t^{\mathrm{at}(2)}\left(\boldsymbol{p} ; \boldsymbol{p}^{\prime}\right) \simeq-\frac{\mathrm{i}}{2} \frac{p_{a_{1}}+p_{a_{1}}^{\prime}}{(2 \pi)^{2} m \hbar} \int_{\mathbb{R}} \mathrm{d} a_{2} \mathrm{e}^{-\mathrm{i} \Delta p_{a_{2}} a_{2} / \hbar}\left[1-\tau_{a}^{\mathrm{at}}\left(\boldsymbol{p}^{\prime} ; a_{2}\right)\right] .
$$

Die Transmissionsfunktion des Steges ist darin abschnittsweise definiert durch

$$
\tau_{a}^{\text {at }}\left(\boldsymbol{p}^{\prime} ; a_{2}\right):=\left\{\begin{array}{ll}
0 & :-\frac{A}{2}<a_{2}<\frac{A}{2} \\
\exp \left[\mathrm{i} \phi_{a}\left(\boldsymbol{p}^{\prime} ; a_{2}\right)\right] & : \text { sonst }
\end{array} .\right.
$$

\subsubsection{Atombeugung am Gitter bei nicht-senkrechtem Einfall}

In bisherigen Arbeiten zur Teilchenbeugung am Transmissionsgitter erwies sich das Konzept der „effektiven Spaltbreite“ als sehr fruchtbar, das die attraktive Wechselwirkung [18] und Größeneffekte [5] auf anschauliche Weise mit einer scheinbaren Verringerung der geometrischen Spaltbreite des Beugungsgitters in Verbindung brachte. Ein entsprechendes Konzept ist deswegen auch für nicht-senkrechten Einfall wünschenswert. Während jedoch die geometrische Spaltbreite eines Gitters bei senkrechtem Einfall natürlicherweise als der kürzeste Abstand $s_{0}$ zweier benachbarter Stege definierbar ist, ist eine äquivalente Definition bei nicht-senkrechtem Einfall mit teilweise abgeschattetem Spalt nicht offensichtlich. In diesem Abschnitt wird gezeigt, daß die Übergangsamplitude des Steges (2.27) natürlicherweise die Wahl der in Abbildung 2.3 eingezeichneten diagonalen Strecke $S_{0}$ als Spaltbreite bedingt. (In Analogie zum senkrechten Einfall berührt diese Strecke die Randkurven der Stege an den beiden Stellen, wo die dem Teilchenstrahl zugewandten, „beleuchteten“ und abgewandten, ,„abgeschatteten“ Anteile der Randkurven aneinanderstoßen.)

Ausgehend von der Übergangsamplitude des Steges (2.27) werden im folgenden $N$ Stege zu einem periodischen Gitter angeordnet und für die dazwischen entstehenden $N-1$ Spalte eine Spaltamplitude definiert. Zuvor muß die Übergangsamplitude des Steges (2.27) durch einige Umformungen vorbereitet werden. Dazu wird ein weiteres Koordinatensystem $\left(u_{1}, u_{2}\right)$ eingeführt, dessen $u_{2}$-Achse mit der Strecke $S_{0}$ zusammenfällt und dessen Ursprung in der Mitte der Strecke $S_{0}$ liegt, wie in Abbildung 2.3 dargestellt. Der ebenfalls eingezeichnete Winkel $\varphi$ ist ein Geometrieparameter des Gitters und hängt mit dem Winkel $\gamma$ (vgl. Abb. 2.2) über die Gittertiefe $t$ und die Gitterperiode $d$ vermittels der Formel $d=t(\tan \varphi+\tan \gamma)$ zusammen. Die Komponenten des einlaufenden und des auslaufenden Impulses bezüglich des Koordinatensystems $\left(u_{1}, u_{2}\right)$ finden im folgenden häufig Verwendung und erhalten deswegen eigene Symbole: Die Komponenten von $\boldsymbol{p}^{\prime}$ werden bezüglich $\left(u_{1}, u_{2}\right)$ mit $\pi_{1}^{\prime}, \pi_{2}^{\prime}$ bezeichnet. Sie transformieren sich nach der Beziehung

$$
\left(\begin{array}{l}
\pi_{1}^{\prime} \\
\pi_{2}^{\prime}
\end{array}\right)_{u}=\left(\begin{array}{cc}
\sin \varphi & -\cos \varphi \\
\cos \varphi & \sin \varphi
\end{array}\right)\left(\begin{array}{l}
p_{1}^{\prime} \\
p_{2}^{\prime}
\end{array}\right)_{x}
$$

Die Komponenten von $\boldsymbol{p}$ werden mit $\pi_{1}, \pi_{2}$ bezeichnet und ebenfalls nach (2.29) transformiert. Auch wird es sich als nützlich erweisen, die Beziehung zwischen den 


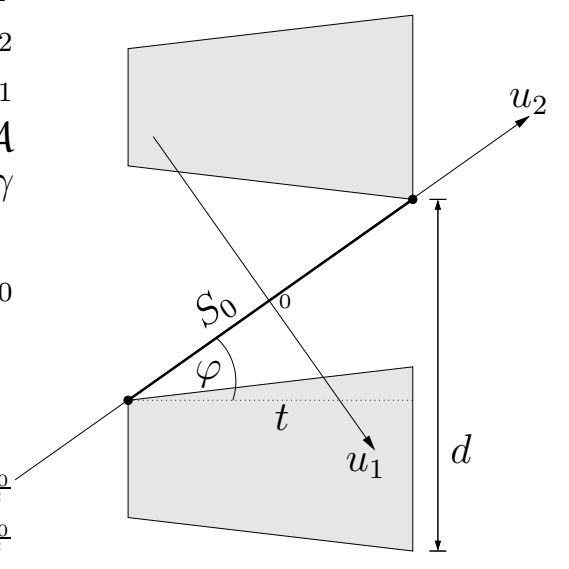

Abbildung 2.3: Die Spaltbreite $S_{0}$ für nicht-senkrechten Einfall zwischen zwei um die Strecke $d$ verschobenen Stegen und das Koordinatensystem $\left(u_{1}, u_{2}\right)$.

Komponenten $\pi_{1}^{\prime}, \pi_{2}^{\prime}$ und $p_{\|}^{\prime}, p_{\perp}^{\prime}$ zu kennen: ersetzt man den Winkel $\varphi$ durch den Winkel

$$
\phi^{\prime}:=\theta^{\prime}-\varphi+\frac{\pi}{2},
$$

um welchen die Koordinatensysteme $\left(x_{\|}, x_{\perp}\right)$ und $\left(u_{1}, u_{2}\right)$ gegeneinander verdreht sind, so findet man

$$
\left(\begin{array}{l}
\pi_{1}^{\prime} \\
\pi_{2}^{\prime}
\end{array}\right)_{u}=\left(\begin{array}{cc}
\cos \phi^{\prime} & -\sin \phi^{\prime} \\
\sin \phi^{\prime} & \cos \phi^{\prime}
\end{array}\right)\left(\begin{array}{l}
p_{\|}^{\prime} \\
p_{\perp}^{\prime}
\end{array}\right)_{\|}
$$

In der Übergangsamplitude des einzelnen Steges (2.27) wird nun die Integrationsvariable $a_{2}$ durch die neue Variable $u_{2}$ nach der Vorschrift $u_{2}:=\frac{\Delta p_{a_{2}}}{\Delta \pi_{2}}\left(a_{2} \pm \frac{A}{2}\right) \pm \frac{S_{0}}{2}$ für $a_{2} \lessgtr 0$ substituiert. Diese Variable kann für $a_{2}>0$ als Position auf der $u_{2^{-}}$ Achse interpretiert werden; für $a_{2}<0$ kann sie als Position auf einer um $-d$ in $x_{2}$-Richtung verschobenen $u_{2}$-Achse gedeutet werden. Nach der Substitution treten die folgenden Ausdrücke auf, die wegen der Energieerhaltung jeweils durch die rechts vom Gleichheitszeichen stehenden Terme ersetzt werden können:

$$
\begin{aligned}
\Delta p_{a_{2}} A+\Delta \pi_{2} S_{0} & =\Delta p_{2} d, \\
\frac{p_{a_{1}}+p_{a_{1}}^{\prime}}{\Delta p_{a_{2}}}=\frac{p_{1}+p_{1}^{\prime}}{\Delta p_{2}} & =\frac{\pi_{1}+\pi_{1}^{\prime}}{\Delta \pi_{2}} .
\end{aligned}
$$

Daraufhin kann eine von $u_{2}$ abhängige Transmissionsfunktion des Spalts durch

$$
\tau_{u}^{\text {at }}\left(\boldsymbol{p}^{\prime} ; u_{2}\right):= \begin{cases}\exp \left[\mathrm{i} \phi_{u}\left(\boldsymbol{p}^{\prime} ; u_{2}\right)\right]: & -\frac{S_{0}}{2}<u_{2}<\frac{S_{0}}{2} \\ 0 & : \text { sonst }\end{cases}
$$

definiert werden, deren Phasenfunktion $\phi_{u}\left(\boldsymbol{p}^{\prime} ; u_{2}\right)$ unter Verwendung von Gleichung (2.25) abschnittsweise durch

$$
\phi_{u}\left(\boldsymbol{p}^{\prime} ; u_{2}\right):=\left\{\begin{array}{l}
\phi_{a}\left(\boldsymbol{p}^{\prime} ; \frac{\pi_{1}+\pi_{1}^{\prime}}{p_{a_{1}}+p_{a_{1}}^{\prime}}\left(u_{2}+\frac{S_{0}}{2}\right)+\frac{A}{2}\right):-\frac{S_{0}}{2}<u_{2}<0 \\
\phi_{a}\left(\boldsymbol{p}^{\prime} ; \frac{\pi_{1}+\pi_{1}^{\prime}}{p_{a_{1}+p_{a_{1}}^{\prime}}}\left(u_{2}-\frac{S_{0}}{2}\right)-\frac{A}{2}\right): 0<u_{2}<\frac{S_{0}}{2}
\end{array}\right.
$$


gegeben ist. Mit der Bezeichnung $D:=d \Delta p_{2} / \Delta \pi_{2}$ folgt aus Gleichung (2.27) sodann

$$
\begin{aligned}
& t^{\mathrm{at}(2)}\left(\boldsymbol{p} ; \boldsymbol{p}^{\prime}\right) \simeq-\frac{\mathrm{i}}{2} \frac{\pi_{1}+\pi_{1}^{\prime}}{(2 \pi)^{2} m \hbar}\left\{\mathrm{e}^{\mathrm{i} \Delta p_{2} d / 2 \hbar} \int_{-\infty}^{\frac{D}{2}} \mathrm{~d} u_{2} \mathrm{e}^{-\mathrm{i} \Delta \pi_{2} u_{2} / \hbar}\left[1-\tau_{u}^{\mathrm{at}}\left(\boldsymbol{p}^{\prime} ; u_{2}\right)\right]\right. \\
&\left.+\mathrm{e}^{-\mathrm{i} \Delta p_{2} d / 2 \hbar} \int_{-\frac{D}{2}}^{\infty} \mathrm{d} u_{2} \mathrm{e}^{-\mathrm{i} \Delta \pi_{2} u_{2} / \hbar}\left[1-\tau_{u}^{\mathrm{at}}\left(\boldsymbol{p}^{\prime} ; u_{2}\right)\right]\right\} .
\end{aligned}
$$

Um die beiden Terme in den geschweiften Klammern in Gleichung (2.34) später als Integrationen über jeweils einen halben Gitterspalt interpretieren zu können, müssen die unendlichen Integrationsgrenzen durch $u_{2}=0$ ersetzt werden. Dies ist wegen der Annahme der Kurzreichweitigkeit von $W_{\text {att }}$ bei nicht zu großem Einfallswinkel in guter Näherung möglich, denn wie die explizite Berechnung in Anhang A zeigt, fällt die Phasenfunktion $\phi_{u}\left(\boldsymbol{p}^{\prime} ; u_{2}\right)$ mit dem Abstand von der Stegoberfläche schnell ab und werden die Integranden in (2.34) vernachlässigbar klein. Für eine Abschätzung
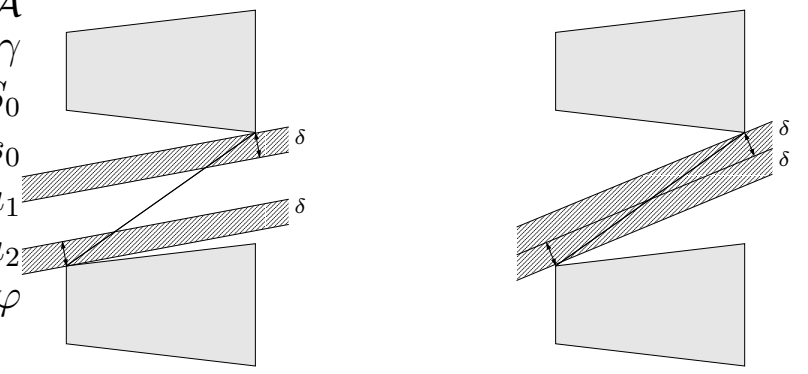

Abbildung 2.4: Bei zu großem Einfallswinkel überlappen am Gitter die Bereiche, in denen die attraktive Wechselwirkung berücksichtigt werden muß.

sei angenommen, daß die Reichweite von $W_{\text {att }}$ auf einen senkrechten Abstand $\delta$ von der Stegoberfläche begrenzt ist. Wie in Abbildung 2.4 skizziert darf der Einfallswinkel am Gitter nicht zu groß gewählt werden, da andernfalls die Reichweiten benachbarter Stege überlappen. Eine geometrische Überlegung führt auf die Forderung

$$
\theta^{\prime}<\varphi-\arcsin \left(2 \delta / S_{0}\right)
$$

Für typische Werte $S_{0}=1400 \AA$ und $\varphi=0,56 \mathrm{rad}$ (vgl. Kap. 4) sowie für die bei Helium-Atomen im Grundzustand ausreichende Annahme $\delta=10 \mathrm{~nm}$ findet man beispielsweise $\theta^{\prime}<24^{\circ}$.

Nun können mehrere Stege zu einem periodischen Gitter kombiniert werden. Ein $n$-fach um den Vektor $\boldsymbol{d}$ verschobener Gittersteg wird durch das Potential $W(\boldsymbol{x}-n \boldsymbol{d})$ beschrieben. Seine Übergangsamplitude ist daher relativ zum unverschobenen Steg durch

$$
\mathrm{e}^{-\mathrm{i} n \Delta \boldsymbol{p} \cdot \boldsymbol{d} / \hbar} t^{\mathrm{at}(2)}\left(\boldsymbol{p} ; \boldsymbol{p}^{\prime}\right)
$$

gegeben. Unter der Voraussetzung der Beugungsbedingung (2.10) kann die Übergangsamplitude eines aus $N$ um den Vektor $\boldsymbol{d}=d \boldsymbol{e}_{2}$ relativ zueinander verschobenen Stegen bestehenden Gitters näherungsweise als kohärente Summe aller $N$ einzelnen 
Stegamplituden [40] berechnet werden:

$$
t_{\text {git }}^{\text {at }(2)}\left(\boldsymbol{p} ; \boldsymbol{p}^{\prime}\right) \simeq \sum_{n=0}^{N-1} \mathrm{e}^{-\mathrm{i}\left(n-\frac{1}{2}(N-1)\right) \Delta p_{2} d / \hbar} t^{\mathrm{at}(2)}\left(\boldsymbol{p} ; \boldsymbol{p}^{\prime}\right) .
$$

Einsetzen der umgeformten Übergangsamplitude des Einzelsteges (2.34) und Umsortieren der Summanden erlaubt, die Integrationsgrenzen bei $u_{2}=0$ von benachbarten Stegen zu identifizieren. So folgt für die Übergangsamplitude des Gitters bei nichtsenkrechtem Einfall der Ausdruck

$$
\begin{aligned}
t_{\text {git }}^{\text {at }(2)}\left(\boldsymbol{p} ; \boldsymbol{p}^{\prime}\right) & \simeq-\frac{\mathrm{i}}{2} \frac{\pi_{1}+\pi_{1}^{\prime}}{(2 \pi)^{2} m \hbar} \\
\times & \left\{\sum_{n=0}^{N-2} \mathrm{e}^{-\mathrm{i}\left(n-\frac{1}{2}(N-2)\right) \Delta p_{2} d / \hbar} \int_{-\frac{D}{2}}^{\frac{D}{2}} \mathrm{~d} u_{2} \mathrm{e}^{-\mathrm{i} \Delta \pi_{2} u_{2} / \hbar}\left[1-\tau_{u}^{\mathrm{at}}\left(\boldsymbol{p}^{\prime} ; u_{2}\right)\right]\right. \\
& +\mathrm{e}^{\mathrm{i} N \Delta p_{2} d / 2 \hbar} \int_{0}^{\frac{D}{2}} \mathrm{~d} u_{2} \mathrm{e}^{-\mathrm{i} \Delta \pi_{2} u_{2} / \hbar}\left[1-\tau_{u}^{\mathrm{at}}\left(\boldsymbol{p}^{\prime} ; u_{2}\right)\right] \\
& \left.+\mathrm{e}^{-\mathrm{i} N \Delta p_{2} d / 2 \hbar} \int_{-\frac{D}{2}}^{0} \mathrm{~d} u_{2} \mathrm{e}^{-\mathrm{i} \Delta \pi_{2} u_{2} / \hbar}\left[1-\tau_{u}^{\mathrm{at}}\left(\boldsymbol{p}^{\prime} ; u_{2}\right)\right]\right\} .
\end{aligned}
$$

Der erste Term in den geschweiften Klammern kann nun als kohärente Beugung von den zwischen den $N$ Stegen entstandenen $N-1$ Spalten interpretiert werden, die anderen beiden als Korrektur von den Rändern des Gitters. Die Summe über $n$ kann ausgeführt werden. Sie liefert die „Gitterfunktion“

$$
H_{N}\left(\Delta p_{2}\right):=\frac{\sin \left(\Delta p_{2} d N / 2 \hbar\right)}{\sin \left(\Delta p_{2} d / 2 \hbar\right)}
$$

zum Index $N-1$. Gleichung (2.38) gibt außerdem Anlaß zur Definition der Spaltamplitude für nicht-senkrechten Einfall durch

$$
a_{u}^{\text {at }}\left(\boldsymbol{p}^{\prime} ; \Delta \pi_{2}\right):=\int_{-\frac{S_{0}}{2}}^{\frac{S_{0}}{2}} \mathrm{~d} u_{2} \exp \left(-\mathrm{i} \Delta \pi_{2} u_{2} / \hbar\right) \tau_{u}^{\text {at }}\left(\boldsymbol{p}^{\prime} ; u_{2}\right),
$$

wobei ausgenutzt wurde, daß die Transmissionsfunktion des Spalts nach ihrer Definition für $\left|u_{2}\right|>\frac{S_{0}}{2}$ verschwindet. Die Spaltamplitude hat, sieht man von einer kleinen Korrektur durch die attraktive Wechselwirkung ab, ein Maximum der Breite $\Delta \pi_{2}=4 \pi \hbar / S_{0}$ um die Vorwärtsrichtung. Die Übergangsamplitude des Gitters kann nun geschrieben werden als

$$
\begin{aligned}
& t_{\text {git }}^{\mathrm{at}(2)}\left(\boldsymbol{p} ; \boldsymbol{p}^{\prime}\right) \simeq-\frac{\mathrm{i}}{2} \frac{\pi_{1}+\pi_{1}^{\prime}}{(2 \pi)^{2} m \hbar} \\
& \times\left\{\frac{\Delta p_{2}}{\Delta \pi_{2}} \frac{\sin \left(\Delta p_{2} N\left[1-\frac{S_{0}}{N D}\right] d / 2 \hbar\right)}{\Delta p_{2} / 2 \hbar}-H_{N-1}\left(\Delta p_{2}\right) a_{u}^{\mathrm{at}}\left(\boldsymbol{p}^{\prime} ; \Delta \pi_{2}\right)\right. \\
& \quad+\mathrm{e}^{\mathrm{i} N \Delta p_{2} d / 2 \hbar} \int_{0}^{\frac{S_{0}}{2}} \mathrm{~d} u_{2} \mathrm{e}^{-\mathrm{i} \Delta \pi_{2} u_{2} / \hbar}\left[1-\tau_{u}^{\mathrm{at}}\left(\boldsymbol{p}^{\prime} ; u_{2}\right)\right]
\end{aligned}
$$




$$
\left.+\mathrm{e}^{-\mathrm{i} N \Delta p_{2} d / 2 \hbar} \int_{-\frac{S_{0}}{2}}^{0} \mathrm{~d} u_{2} \mathrm{e}^{-\mathrm{i} \Delta \pi_{2} u_{2} / \hbar}\left[1-\tau_{u}^{\mathrm{at}}\left(\boldsymbol{p}^{\prime} ; u_{2}\right)\right]\right\} .
$$

Der erste Summand in den geschweiften Klammern besitzt für große $N$ ein proportional zu $N$ anwachsendes, scharfes Maximum bei $\Delta p_{2}=0$ und konvergiert im Limes $N \rightarrow \infty$ gegen $2 \pi \hbar \frac{\Delta p_{2}}{\Delta \pi_{2}} \delta\left(\Delta p_{2}\right)$. Die Gitterfunktion im zweiten Summanden besitzt proportional zu $N$ anwachsende, scharfe Hauptmaxima an den Stellen $\Delta p_{2}=n 2 \pi \hbar / d$. Die Breite dieser Hauptmaxima ist von der Größenordnung $\Delta p_{2}=4 \pi \hbar / N d$. Im Experiment ist typischerweise $N \approx 100$ realisiert [18], so daß die Spaltamplitude (2.40) auf den Breiten der Hauptmaxima langsam veränderlich ist. Für die Anwendungen ist es daher formal möglich, den Grenzübergang $N \rightarrow \infty$ durchzuführen. Da die Beträge der verbleibenden beiden Summanden in Gleichung (2.41) unabhängig von $N$ sind, werden sie relativ wie $N^{-1}$ unterdrückt. Unter Verwendung der Identität (2.31b) kann die Übergangsamplitude des Gitters bei nichtsenkrechtem Einfall daher näherungsweise geschrieben werden als

$$
t_{\mathrm{git}}^{\mathrm{at}(2)}\left(\boldsymbol{p} ; \boldsymbol{p}^{\prime}\right) \simeq-\frac{\mathrm{i}}{2} \frac{\pi_{1}+\pi_{1}^{\prime}}{(2 \pi)^{2} m \hbar}\left\{2 \pi \hbar \frac{p_{1}}{\pi_{1}} \delta\left(\Delta p_{2}\right)-H_{N}\left(\Delta p_{2}\right) a_{u}^{\text {at }}\left(\boldsymbol{p}^{\prime} ; \Delta \pi_{2}\right)\right\},
$$

wobei der Limes der Gitterfunktion durch

$$
H_{N}\left(\Delta p_{2}\right) \stackrel{N \rightarrow \infty}{\rightarrow} \frac{2 \pi \hbar}{d} \sum_{n^{\prime}=-\infty}^{\infty} \delta\left(\Delta p_{2}-\frac{n^{\prime} 2 \pi \hbar}{d}\right)
$$

gegeben ist. Aufgrund der Energieerhaltung ist nur eine endliche Anzahl von Hauptmaxima beobachtbar.

\subsubsection{Das Beugungsbild des Atoms}

Im folgenden wird die Intensität der Beugungsordnungen eines Gitters bei nichtsenkrechtem Einfall berechnet. Gleichung (2.4) gibt die Wahrscheinlichkeitsdichte dafür an, ein mit der Impulsverteilung $\psi\left(\mathbf{p}^{\prime}\right)$ einlaufendes Atom unter dem auslaufenden Impuls $\mathbf{p} \equiv \mathbf{p}^{\prime}+\Delta \mathbf{p}$ zu messen. Im Experiment sollte die Impulsverteilung scharf um einen mittleren einlaufenden Impuls $\overline{\mathbf{p}}^{\prime}$ konzentriert sein, so daß benachbarte Beugungsordnungen auflösbar sind und daß die Spaltamplitude auf der Breite der Impulsverteilung langsam veränderlich ist. Es wird im folgenden vorausgesetzt, daß diese Forderungen erfüllt sind. Einsetzen der Übergangsamplitude (2.42) in das Streumatrixelement (2.5) führt auf den Ausdruck

$$
\begin{aligned}
w(\mathbf{p} ; \psi)=\mid \int \mathrm{d}^{3} p^{\prime} \psi\left(\mathbf{p}^{\prime}\right)\left[\delta^{(3)}\right. & (\Delta \mathbf{p})-\frac{\pi_{1}+\pi_{1}^{\prime}}{4 \pi m \hbar} \delta\left(\Delta p_{3}\right) \delta\left(E-E^{\prime}\right) \\
\times & \left.\left\{2 \pi \hbar \frac{p_{1}}{\pi_{1}} \delta\left(\Delta p_{2}\right)-H_{N}\left(\Delta p_{2}\right) a_{u}^{\text {at }}\left(\boldsymbol{p}^{\prime} ; \Delta \pi_{2}\right)\right\}\right]\left.\right|^{2}
\end{aligned}
$$

für die Detektionswahrscheinlichkeitsdichte (2.4). Ausmultiplizieren der geschweiften Klammer liefert drei Terme, von denen zunächst der mittlere betrachtet werden soll: 
nach dem Einsetzen von $E^{\prime}=\left|\mathbf{p}^{\prime}\right|^{2} / 2 m$ und $E=|\mathbf{p}|^{2} / 2 m$ kann er vereinfacht werden $\mathrm{zu}$

$$
-\frac{\pi_{1}+\pi_{1}^{\prime}}{2 \pi_{1}} \frac{p_{1}}{\left|p_{1}\right|} \delta\left(\Delta p_{2}\right) \delta\left(\Delta p_{3}\right)\left[\delta\left(\Delta p_{1}\right)+\delta\left(p_{1}^{\prime}+p_{1}\right)\right] .
$$

Von den beiden Deltafunktionen in eckigen Klammern führt $\delta\left(\Delta p_{1}\right)$ gerade zur Kompensation des Terms $\delta^{(3)}(\Delta \mathbf{p})$ aus Gleichung (2.44). Wegen der geforderten scharfen Impulsverteilung ist darüberhinaus der Beitrag $\delta\left(p_{1}^{\prime}+p_{1}\right)$ im Bereich des Beugungsbildes vernachlässigbar. Der verbleibende Ausdruck für die Wahrscheinlichkeitsdichte (2.44) lautet also

$$
w(\mathbf{p} ; \psi) \simeq\left|\int \mathrm{d}^{3} p^{\prime} \psi\left(\mathbf{p}^{\prime}\right) \frac{\pi_{1}+\pi_{1}^{\prime}}{4 \pi m \hbar} \delta\left(\Delta p_{3}\right) \delta\left(E-E^{\prime}\right) H_{N}\left(\Delta p_{2}\right) a_{u}^{\text {at }}\left(\boldsymbol{p}^{\prime} ; \Delta \pi_{2}\right)\right|^{2} .
$$

Wegen der Konzentration der Spaltamplitude auf kleine Beugungswinkel um die Vorwärtsrichtung ist es vorteilhaft, die Impulse wieder durch ihre Komponenten parallel und senkrecht zum mittleren einfallenden Impuls $\overline{\boldsymbol{p}}^{\prime}$ auszudrücken. Bezeichnet $\overline{\theta^{\prime}}$ den zu $\overline{\boldsymbol{p}}^{\prime}$ gehörenden mittleren Einfallswinkel, so lautet die Transformation der Komponenten eines beliebigen Vektors $\boldsymbol{v}$ in der Ebene

$$
\left(\begin{array}{l}
v_{1} \\
v_{2}
\end{array}\right)_{x}=\left(\begin{array}{cc}
\cos \overline{\theta^{\prime}} & -\sin \overline{\theta^{\prime}} \\
\sin \overline{\theta^{\prime}} & \cos \overline{\theta^{\prime}}
\end{array}\right)\left(\begin{array}{c}
v_{\|} \\
v_{\perp}
\end{array}\right)_{\|} .
$$

Per Konstruktion gilt insbesondere $\bar{p}_{\|}^{\prime}=\left|\overline{\boldsymbol{p}}^{\prime}\right|$ und $\bar{p}_{\perp}^{\prime}=0$. Das Produkt der Deltafunktionen in Gleichung (2.45) läßt sich nun schreiben als

$$
\delta\left(\Delta p_{3}\right) \delta\left(E-E^{\prime}\right)=\delta\left(\Delta p_{3}\right) \frac{m}{\left|p_{\|}^{\prime}\right|}\left[\delta\left(p_{\|}^{\prime}-\left(1-\alpha\left(p_{\perp}^{\prime}\right)\right)\left|p_{\|}\right|\right)+\delta\left(p_{\|}^{\prime}+\left(1-\alpha\left(p_{\perp}^{\prime}\right)\right)\left|p_{\|}\right|\right)\right]
$$

mit

$$
1-\alpha\left(p_{\perp}^{\prime}\right):=\sqrt{1+\frac{p_{\perp}^{2}-p_{\perp}^{\prime 2}}{p_{\|}^{2}}},
$$

wobei in (2.48) die positive Wurzel zu verwenden ist. Im Integranden der Wahrscheinlichkeitsdichte (2.45) gilt $\left|\alpha\left(p_{\perp}^{\prime}\right)\right| \ll 1$. Die scharfe Impulsverteilung $\psi\left(\mathbf{p}^{\prime}\right)$ erlaubt daher wiederum, den Beitrag von $\delta\left(p_{\|}^{\prime}+\left(1-\alpha\left(p_{\perp}^{\prime}\right)\right)\left|p_{\|}\right|\right)$zu vernachlässigen und die Betragsstriche an $p_{\|}^{\prime}$ und $p_{\|}$fortzulassen. Ausintegrieren von $p_{\|}^{\prime}$ und $p_{3}^{\prime}$ in Gleichung (2.45) führt anschließend auf

$$
\begin{aligned}
w(\mathbf{p} ; \psi) \simeq \mid \frac{1}{4 \pi \hbar} \int \mathrm{d} p_{\perp}^{\prime} & {\left[\psi\left(p_{\|}^{\prime}, p_{\perp}^{\prime}, p_{3}\right) \frac{\pi_{1}+\pi_{1}^{\prime}}{p_{\|}^{\prime}}\right.} \\
& \left.\times H_{N}\left(\Delta p_{2}\right) a_{u}^{\text {at }}\left(\boldsymbol{p}^{\prime} ; \Delta \pi_{2}\right)\right]\left._{p_{\|}^{\prime}=\left[1-\alpha\left(p_{\perp}^{\prime}\right)\right] p_{\|}}\right|^{2} .
\end{aligned}
$$

An dieser Stelle ist es hilfreich, die Gitterfunktion $H_{N}\left(\Delta p_{2}\right)$ durch ihren Limes (2.43) zu ersetzen. Der Transformation (2.46) zufolge gilt

$$
\Delta p_{2}=\Delta p_{\|} \sin \overline{\theta^{\prime}}+\Delta p_{\perp} \cos \overline{\theta^{\prime}},
$$


so daß die Gitterfunktion übergeht in

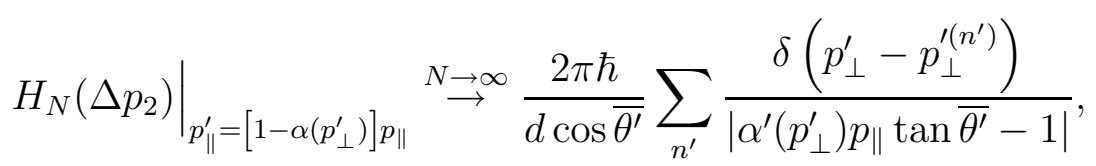

wobei $\alpha^{\prime}\left(p_{\perp}^{\prime}\right)$ die Ableitung von $\alpha\left(p_{\perp}^{\prime}\right)$ nach $p_{\perp}^{\prime}$ bezeichnet, und die Nullstellen des Arguments der Deltafunktionen $p_{\perp}^{\prime}=p_{\perp}^{\left(n^{\prime}\right)}$ zu festem $\boldsymbol{p}$ und $n^{\prime}$ durch

$$
p_{\perp}^{\left(n^{\prime}\right)}:=-\sin \overline{\theta^{\prime}} \sqrt{|\boldsymbol{p}|^{2}-\left[p_{2}-\frac{n^{\prime} 2 \pi \hbar}{d}\right]^{2}}+\cos \overline{\theta^{\prime}}\left[p_{2}-\frac{n^{\prime} 2 \pi \hbar}{d}\right]
$$

gegeben sind. In (2.52) ist ebenfalls die positive Wurzel zu verwenden. Die Ausintegration von $p_{\perp}^{\prime}$ in (2.49) führt nun zu der Darstellung

$$
\begin{aligned}
& w(\mathbf{p} ; \psi) \simeq \mid \frac{1}{2 d \cos \overline{\theta^{\prime}}} \sum_{n^{\prime}}\left[\psi\left(p_{\|}^{\prime}, p_{\perp}^{\prime}, p_{3}\right) \frac{\pi_{1}+\pi_{1}^{\prime}}{p_{\|}^{\prime}}\right. \\
& \left.\times \frac{1}{\left|\alpha^{\prime}\left(p_{\perp}^{\prime}\right) p_{\|} \tan \overline{\theta^{\prime}}-1\right|} a_{u}^{\text {at }}\left(\boldsymbol{p}^{\prime} ; \Delta \pi_{2}\right)\right]\left.\right|_{\substack{\left.p_{\perp}^{\prime}=p_{\perp}^{\prime\left(n^{\prime}\right)} \\
p_{\|}^{\prime}=\left[1-\alpha\left(p_{\perp}^{\prime\left(n^{\prime}\right)}\right)\right]\right]_{\|\|}}} ^{2} .
\end{aligned}
$$

Wegen der vorausgesetzen Konzentration der Impulsverteilung $\psi$ dürfen die übrigen Faktoren in den eckigen Klammern näherungsweise beim mittleren Impuls $\overline{\boldsymbol{p}}^{\prime}$ ausgewertet werden.

$$
\begin{aligned}
p_{\perp}^{\prime} & =p_{\perp}^{\prime\left(n^{\prime}\right)} \approx \bar{p}_{\perp}^{\prime} \equiv 0 \\
p_{\|}^{\prime} & =\left[1-\alpha\left(p_{\perp}^{\prime\left(n^{\prime}\right)}\right)\right] p_{\|} \approx \bar{p}_{\|}^{\prime}=\left|\overline{\boldsymbol{p}}^{\prime}\right|
\end{aligned}
$$

Im Nenner von Gleichung (2.53) gilt $\alpha^{\prime}\left(\bar{p}_{\perp}^{\prime}\right)=\bar{p}_{\perp}^{\prime} /\left(p_{\|} \bar{p}_{\|}^{\prime}\right)=0$. So ergibt sich

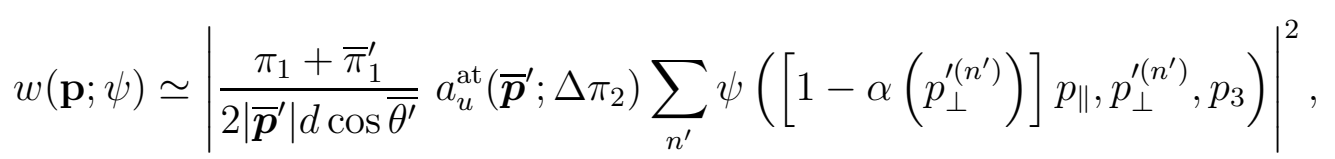

wobei auch die Impulskomponenten $\bar{\pi}_{k}^{\prime}$ beim mittleren einlaufenden Impuls $\overline{\boldsymbol{p}}^{\prime}$ auszuwerten sind. Diese Gleichung zeigt, daß die Wahrscheinlichkeitsdichte $w(\mathbf{p} ; \psi)$ bei solchen auslaufenden Impulsen scharfe Maxima besitzt, die für ein $n$ die Nullstellenbedingung (2.52) zu $p_{\perp}^{\prime(n)}=0$ erfüllen. Nach (2.54) gilt an diesen Stellen $|\boldsymbol{p}|=\left|\overline{\boldsymbol{p}}^{\prime}\right|$. Beides kombinierend erhält man, für den auslaufenden Impuls die Möglichkeiten

$$
p_{2}=\bar{p}_{2}^{\prime}+\frac{n 2 \pi \hbar}{d}
$$

wobei $p_{1}$ jeweils aus der Energieerhaltung folgt. Dies ist das aufgrund der Periodizität des Transmissionsgitters erwartete Resultat. Der zugehörige Beugungswinkel $\theta_{n}$ mit $p_{2} /|\boldsymbol{p}|=\sin \theta_{n}$ ist bestimmt durch die Gleichung

$$
\sin \theta_{n}=\sin \overline{\theta^{\prime}}+\frac{n 2 \pi \hbar}{\left|\overline{\boldsymbol{p}}^{\prime}\right| d} .
$$


Die endliche Breite der Impulsverteilung $\psi$ verursacht eine Verbreiterung der Beugungsmaxima. Die experimentell bestimmbare Intensität $I_{n}$ des $n$. Beugungsmaximums ist daher zu ermitteln durch Integration der Wahrscheinlichkeitsdichte (2.55) über einen Kegel $\mathcal{C}_{n}$ im Impulsraum, der um den auslaufenden Impuls p zentriert ist und das verbreiterte Maximum einschließt [40]. Man erhält schließlich

$$
I_{n} \propto\left(\frac{\pi_{1}+\bar{\pi}_{1}^{\prime}}{2\left|\overline{\boldsymbol{p}}^{\prime}\right| d \cos {\overline{\theta^{\prime}}}^{\prime}}\right)^{2}\left|a_{u}^{\text {at }}\left(\overline{\boldsymbol{p}}^{\prime} ; \Delta \pi_{2}\right)\right|^{2}
$$

wobei die Komponenten $\bar{\pi}_{1}^{\prime}, \bar{\pi}_{2}^{\prime}$ beim mittleren einlaufenden Impuls $\overline{\boldsymbol{p}}^{\prime}$ und die Komponenten $\pi_{1}, \pi_{2}$ beim durch (2.56) festgelegten auslaufenden Impuls $\boldsymbol{p}$ auszuwerten sind. Die Spaltamplitude ist also die Einhüllende des Beugungsbildes. Die Breite ihres zentralen Maximums $\Delta \pi_{2} \approx 4 \pi \hbar / S_{0}$ kann mit Gleichung (2.56) auch als Anzahl darin enthaltener Beugungsordnungen ausgedrückt werden: wegen $\Delta p_{\|}^{\prime} \ll \Delta p_{\perp}^{\prime}$ sind es jeweils $n_{c} \approx d \cos \overline{\theta^{\prime}} / S_{0} \cos \phi^{\prime}=d /\left(s_{0}+t\left(\tan \beta-\tan \overline{\theta^{\prime}}\right)\right)$ zu beiden Seiten der Vorwärtsrichtung.

\section{Darstellung der Beugungsintensitäten durch Kumulanten}

Vorarbeiten zeigten, daß der direkte Vergleich der Intensitätsformel (2.58) mit experimentellen Beugungsintensitäten zur Anpassung der Geometrieparameter des Transmissionsgitters numerisch instabil ist. Dieser Schwierigkeit kann durch Approximation der Intensitätsformel durch einen einfacheren, analytischen Ausdruck begegnet werden. Dazu wird eine Kumulantenentwicklung der Spaltamplitude (2.40) berechnet. Zunächst wird die Spaltamplitude in der folgenden Form geschrieben:

$$
\begin{aligned}
a_{u}^{\text {at }}\left(\boldsymbol{p}^{\prime} ; \Delta \pi_{2}\right)= & \int_{-S_{0} / 2}^{0} \mathrm{~d} u_{2} \exp \left(-\mathrm{i} \Delta \pi_{2} u_{2} / \hbar\right) \tau_{u}^{\text {at }}\left(\boldsymbol{p}^{\prime} ; u_{2}\right) \\
& +\int_{0}^{S_{0} / 2} \mathrm{~d} u_{2} \exp \left(-\mathrm{i} \Delta \pi_{2} u_{2} / \hbar\right) \tau_{u}^{\text {at }}\left(\boldsymbol{p}^{\prime} ; u_{2}\right) .
\end{aligned}
$$

Im ersten Integral wird die Substitution $\xi:=\frac{S_{0}}{2}+u_{2}$ und im zweiten $\xi:=\frac{S_{0}}{2}-u_{2}$ durchgeführt. Durch partielle Integration über $\xi$ erhält man sodann

$$
\begin{aligned}
a_{u}^{\mathrm{at}}\left(\boldsymbol{p}^{\prime} ; \Delta \pi_{2}\right)=\frac{\hbar}{\mathrm{i} \Delta \pi_{2}}\left\{\mathrm{e}^{\mathrm{i} \Delta \pi_{2} S_{0} / 2 \hbar}(\right. & {\left[-\mathrm{e}^{-\mathrm{i} \Delta \pi_{2} \xi / \hbar} \tau_{u}^{\mathrm{at}}\left(\boldsymbol{p}^{\prime} ; \xi-\frac{S_{0}}{2}\right)\right]_{0}^{S_{0} / 2} } \\
& \left.+\int_{0}^{S_{0} / 2} \mathrm{~d} \xi \mathrm{e}^{-i \Delta \pi_{2} \xi / \hbar} \tau_{u}^{\mathrm{at}{ }^{\prime}}\left(\boldsymbol{p}^{\prime} ; \xi-\frac{S_{0}}{2}\right)\right) \\
+\mathrm{e}^{-\mathrm{i} \Delta \pi_{2} S_{0} / 2 \hbar}\left(\left[\mathrm{e}^{\mathrm{i} \Delta \pi_{2} \xi / \hbar} \tau_{u}^{\mathrm{at}}\left(\boldsymbol{p}^{\prime} ; \frac{S_{0}}{2}-\xi\right)\right]_{0}^{S_{0} / 2}\right. & \\
& \left.\left.+\int_{0}^{S_{0} / 2} \mathrm{~d} \xi \mathrm{e}^{i \Delta \pi_{2} \xi / \hbar} \tau_{u}^{\text {at' }}\left(\boldsymbol{p}^{\prime} ; \frac{S_{0}}{2}-\xi\right)\right)\right\},
\end{aligned}
$$


wobei $\tau_{u}^{\text {at' }}$ die Ableitung von $\tau_{u}^{\text {at }}$ nach dem Ortsargument bezeichnet. Die Randterme der partiellen Integration bei $\xi=\frac{S_{0}}{2}$ heben sich gegenseitig heraus; es bleiben die Randterme bei $\xi=0 \mathrm{zu}$ betrachten, in denen die Transmissionsfunktion an den beiden Spalträndern ausgewertet wird. Aufgrund des repulsiven Potentials der Gitterstege verschwindet aber der atomare Streuzustand auf der Stegoberfläche; die Transmissionfunktion (2.32) sollte dort folglich Null gesetzt werden. Die verbleibenden Terme werden ausgedrückt mit Hilfe der Funktionen

$$
\Phi^{ \pm}(\kappa):=\mp\left[\tau_{u}^{\text {at }}\left(\boldsymbol{p}^{\prime} ; 0\right)\right]^{-1} \int_{0}^{S_{0} / 2} \mathrm{~d} \xi \mathrm{e}^{ \pm \mathrm{i} \kappa \xi} \tau_{u}^{\text {at' }}\left(\boldsymbol{p}^{\prime} ; \pm\left(\frac{S_{0}}{2}-\xi\right)\right)
$$

die $\Phi^{ \pm}(0)=1$ erfüllen. Unter der Bedingung (2.35) gilt $\tau_{u}^{\text {at }}\left(\boldsymbol{p}^{\prime} ; 0\right) \simeq 1$, und es folgt

$$
a_{u}^{\mathrm{at}}\left(\boldsymbol{p}^{\prime} ; \Delta \pi_{2}\right) \simeq \frac{\hbar}{\mathrm{i} \Delta \pi_{2}}\left\{\mathrm{e}^{\mathrm{i} \Delta \pi_{2} S_{0} / 2 \hbar} \Phi^{-}\left(\frac{\Delta \pi_{2}}{\hbar}\right)-\mathrm{e}^{-\mathrm{i} \Delta \pi_{2} S_{0} / 2 \hbar} \Phi^{+}\left(\frac{\Delta \pi_{2}}{\hbar}\right)\right\} .
$$

Analog zu dem Vorgehen in Ref. [18] werden die Funktionen $\Phi^{ \pm}(\kappa)$ nach Kumulanten $R_{j}^{ \pm}$entwickelt mit dem Ziel, diese Entwicklung nach den führenden zwei Termen abzubrechen. Die definierende Relation für die komplexen Zahlen $R_{j}^{ \pm}$lautet

$$
\ln \Phi^{ \pm}(\kappa)=: \sum_{j=1}^{\infty} \frac{( \pm \mathrm{i} \kappa)^{j}}{j !} R_{j}^{ \pm}= \pm \mathrm{i} \kappa R_{1}^{ \pm}-\frac{1}{2} \kappa^{2} R_{2}^{ \pm}+\mathcal{O}\left(\kappa^{3}\right)
$$

Die ersten Kumulanten $R_{1}^{ \pm}$folgen aus der Beziehung

$$
\begin{aligned}
R_{1}^{ \pm} & = \pm\left.\frac{1}{\mathrm{i}} \frac{\mathrm{d}}{\mathrm{d} \kappa} \ln \Phi^{ \pm}(\kappa)\right|_{\kappa=0} \\
& =\frac{S_{0}}{2}-\int_{0}^{S_{0} / 2} \mathrm{~d} \xi \tau_{u}^{\text {at }}\left(\boldsymbol{p}^{\prime} ; \pm\left(\frac{S_{0}}{2}-\xi\right)\right),
\end{aligned}
$$

und entsprechend können die zweiten Kumulanten $R_{2}^{ \pm}$berechnet werden durch

$$
\begin{aligned}
R_{2}^{ \pm} & =-\left.\frac{\mathrm{d}^{2}}{\mathrm{~d} \kappa^{2}} \ln \Phi^{ \pm}(\kappa)\right|_{\kappa=0} \\
& =\left(\frac{S_{0}}{2}\right)^{2}-\left(R_{1}^{ \pm}\right)^{2}-2 \int_{0}^{S_{0} / 2} \mathrm{~d} \xi \xi \tau_{u}^{\text {at }}\left(\boldsymbol{p}^{\prime} ; \pm\left(\frac{S_{0}}{2}-\xi\right)\right) .
\end{aligned}
$$

Die Funktionen $\Phi^{ \pm}(\kappa)$ und somit auch die Kumulanten $R_{j}^{ \pm}$hängen über die Transmissionfunktion vom einfallenden Impuls $\boldsymbol{p}^{\prime}$ und der Geometrie des Gitters ab. Diese Abhängigkeiten wurden der einfacheren Lesbarkeit halber nicht explizit aufgeführt. In Anhang A werden asymptotische Ausdrücke für die Kumulanten $R_{1}^{ \pm}$und $R_{2}^{ \pm}$abgeleitet, die die Abschätzung ihrer Größenordnung bei vorgegebenem einlaufenden Impuls und Gittergeometrie ermöglichen.

Approximiert durch die ersten beiden Kumulantenterme lautet die Spaltamplitude (2.61) nun

$$
\begin{aligned}
& a_{u}^{\text {at }}\left(\boldsymbol{p}^{\prime} ; \Delta \pi_{2}\right) \simeq \frac{\hbar}{\mathrm{i} \Delta \pi_{2}} \\
& \quad \times\left\{\mathrm{e}^{\mathrm{i}\left(S_{0} / 2-R_{1}^{-}\right) \Delta \pi_{2} / \hbar} \mathrm{e}^{-\frac{1}{2}\left(\Delta \pi_{2} / \hbar\right)^{2} R_{2}^{-}}-\mathrm{e}^{-\mathrm{i}\left(S_{0} / 2-R_{1}^{+}\right) \Delta \pi_{2} / \hbar} \mathrm{e}^{-\frac{1}{2}\left(\Delta \pi_{2} / \hbar\right)^{2} R_{2}^{+}}\right\},
\end{aligned}
$$


beziehungsweise in symmetrischerer Form,

$$
\begin{aligned}
& a_{u}^{\text {at }}\left(\boldsymbol{p}^{\prime} ; \Delta\right.\left.\pi_{2}\right) \simeq \frac{2 \hbar}{\Delta \pi_{2}} \mathrm{e}^{\mathrm{i}\left(R_{1}^{+}-R_{1}^{-}\right) \Delta \pi_{2} / 2 \hbar} \mathrm{e}^{-\frac{1}{2}\left(\Delta \pi_{2} / \hbar\right)^{2}\left(R_{2}^{+}+R_{2}^{-}\right) / 2} \\
& \times \sin \left[\left(S_{0}-R_{1}^{+}-R_{1}^{-}\right) \Delta \pi_{2} / 2 \hbar-\frac{\mathrm{i}}{2}\left(R_{2}^{+}-R_{2}^{-}\right)\left(\Delta \pi_{2} / \hbar\right)^{2} / 2\right] .
\end{aligned}
$$

Dieser Ausdruck gibt Anlaß zur Definition der folgenden reellen Größen, die wie die Kumulanten implizit vom einfallenden Impuls $\boldsymbol{p}^{\prime}$ und der Gittergeometrie abhängen:

$$
\begin{aligned}
S_{\mathrm{eff}} & :=S_{0}-\operatorname{Re}\left(R_{1}^{+}+R_{1}^{-}\right) \\
\Delta & :=\operatorname{Im}\left(R_{1}^{+}+R_{1}^{-}\right) \\
\alpha & :=\operatorname{Im}\left(R_{1}^{+}-R_{1}^{-}\right) \\
\Sigma & :=\sqrt{\frac{1}{2} \operatorname{Re}\left(R_{2}^{+}+R_{2}^{-}\right)} \\
\Omega_{R} & :=\frac{1}{2} \operatorname{Re}\left(R_{2}^{+}-R_{2}^{-}\right) \\
\Omega_{I} & :=\frac{1}{2} \operatorname{Im}\left(R_{2}^{+}-R_{2}^{-}\right)
\end{aligned}
$$

sowie

$$
K\left(\theta_{n}\right):=\frac{\Delta \pi_{2}}{\hbar}=\frac{\left|\boldsymbol{p}^{\prime}\right|}{\hbar}\left(\cos \left(\varphi-\theta_{n}\right)-\cos \left(\varphi-\overline{\theta^{\prime}}\right)\right) .
$$

Der geometrische Vorfaktor der Intensitätsformel (2.58) läßt sich durch den Beugungswinkel ausdrücken:

$$
\frac{\pi_{1}+\bar{\pi}_{1}^{\prime}}{\left|\overline{\boldsymbol{p}}^{\prime}\right|}=\sin \left(\varphi-\theta_{n}\right)+\sin \left(\varphi-\overline{\theta^{\prime}}\right) .
$$

Bildung des Betragsquadrates der Spaltamplitude (2.65) führt auf die durch zwei Kumulantenterme approximierte Intensitätsformel

$$
\begin{aligned}
I_{n} \propto & {\left[\frac{\sin \left(\varphi-\theta_{n}\right)+\sin \left(\varphi-\overline{\theta^{\prime}}\right)}{2 \cos \overline{\theta^{\prime}}}\right]^{2} \exp \left[-\alpha K\left(\theta_{n}\right)\right] \exp \left[-\Sigma^{2} K\left(\theta_{n}\right)^{2}\right] } \\
& \times \frac{\sin ^{2}\left[K\left(\theta_{n}\right) S_{\mathrm{eff}} / 2+K^{2}\left(\theta_{n}\right) \Omega_{I} / 2\right]+\sinh ^{2}\left[K\left(\theta_{n}\right) \Delta / 2+K^{2}\left(\theta_{n}\right) \Omega_{R} / 2\right]}{\left[K\left(\theta_{n}\right) d / 2\right]^{2}} .
\end{aligned}
$$

In den Argumenten der Sinus- und der Sinus hyperbolicus-Funktion treten quadratische Terme in $K^{2}\left(\theta_{n}\right)$ auf. Für typische experimentelle Parameter (vgl. Kap. 4) gilt näherungsweise $K\left(\theta_{n}\right) \approx n 10^{-3} \AA^{-1}$. Die asymptotische Entwicklung der Kumulanten $R_{2}^{ \pm}$(A.22) erlaubt die Abschätzung der Koeffizienten $\Omega_{R} \approx-600 \AA^{2}$ und $\Omega_{I} \approx 600 \AA^{2}$. Die linearen Terme in $K\left(\theta_{n}\right)$ haben aber laut Gleichung (A.18) Koeffizienten der Größenordnung $S_{\text {eff }} \approx 1350 \AA$ und $\Delta \approx-100 \AA$, so daß die quadratischen Korrekturen vernachlässigbar sein sollten. Genaue numerische Rechnungen ${ }^{1}$ bestäti-

\footnotetext{
${ }^{1}$ Konkret findet man beispielsweise bei den aus Kapitel 4 übernommenen Strahlparametern $v^{\prime}=494,9 \mathrm{~m} / \mathrm{s}$ und $\theta^{\prime}=21^{\circ}$, sowie den Gitterparametern $\varphi=0,56197 \mathrm{rad}, S_{0}=1397,5 \AA, \beta=6,7^{\circ}$ und $C_{3}=0,155 \mathrm{meV} \mathrm{nm}^{3}$ für $n=7$, folgende Werte: $K\left(\theta_{7}\right) S_{\text {eff }} / 2=5,7$ und $K\left(\theta_{7}\right)^{2} \Omega_{I} / 2=0,03$; sowie $K\left(\theta_{7}\right) \Delta / 2=-0,47$ und $K\left(\theta_{7}\right)^{2} \Omega_{R} / 2=-0,03$.
} 
gen diese Abschätzung. Die Intensitätsformel vereinfacht sich dadurch zu

$$
\begin{aligned}
I_{n} \propto & {\left[\frac{\sin \left(\varphi-\theta_{n}\right)+\sin \left(\varphi-\overline{\theta^{\prime}}\right)}{2 \cos \overline{\theta^{\prime}}}\right]^{2} \exp \left[-\alpha K\left(\theta_{n}\right)\right] \exp \left[-\Sigma^{2} K\left(\theta_{n}\right)^{2}\right] } \\
& \times \frac{\sin ^{2}\left[K\left(\theta_{n}\right) S_{\text {eff }} / 2\right]+\sinh ^{2}\left[K\left(\theta_{n}\right) \Delta / 2\right]}{\left[K\left(\theta_{n}\right) d / 2\right]^{2}} .
\end{aligned}
$$

Aufgrund des nicht-senkrechten Einfalls ist das Beugungsbild nicht symmetrisch in $n$ : Die Asymmetrie des Vorfaktors $\left[\sin \left(\varphi-\theta_{n}\right)+\sin \left(\varphi-\overline{\theta^{\prime}}\right)\right]^{2}$ ist geometrischer Natur

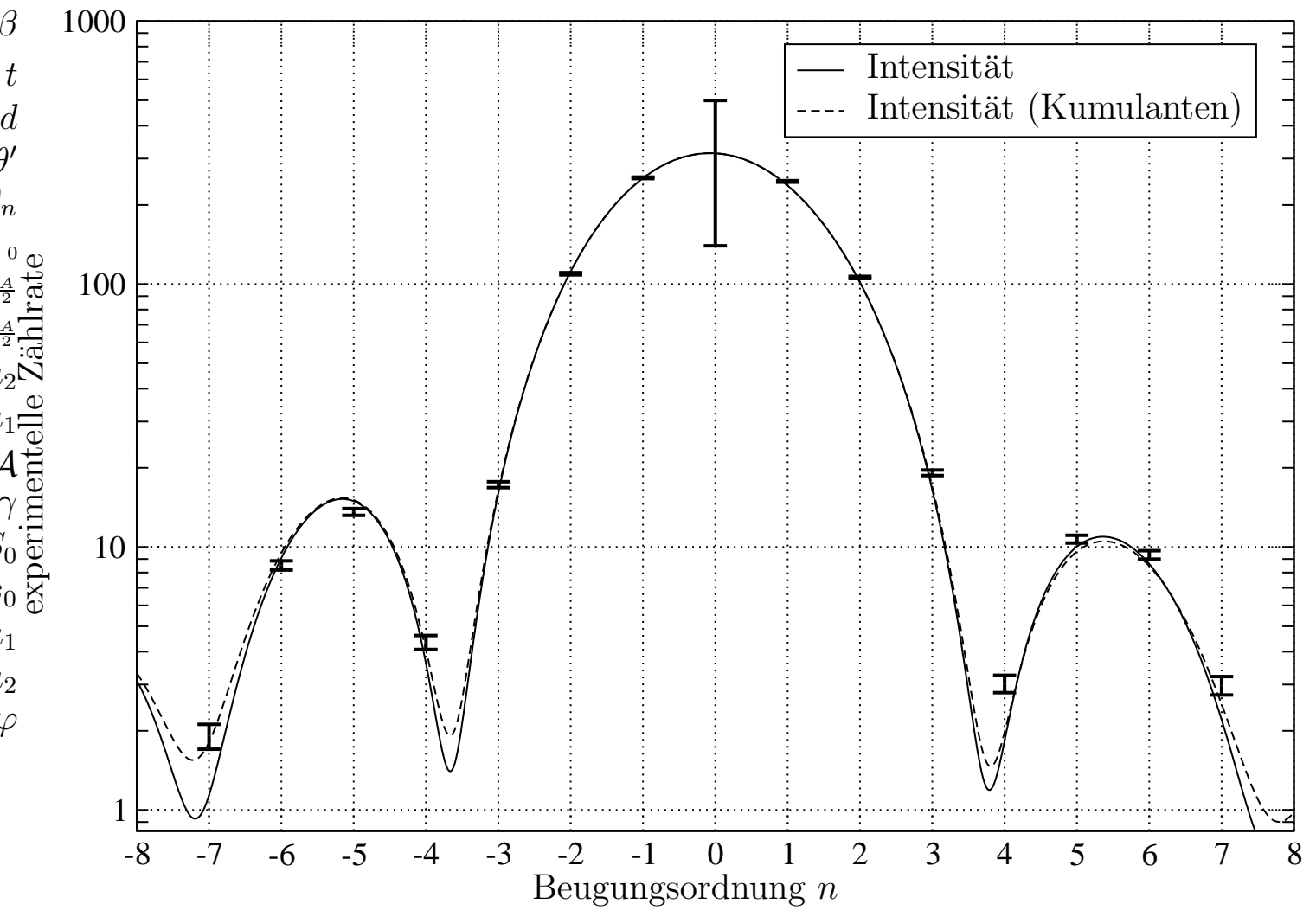

Abbildung 2.5: Vergleich der Intensitätsformel (Gl. 2.58, durchgezogene Kurve) mit der durch zwei Kumulanten approximierten Formel (Gl. 2.75, gestrichelte Kurve). Zur Veranschaulichung wird die Spaltamplitude kontinuierlich dargestellt; experimentell relevant sind aber nur die Werte bei ganzzahligen Beugungsordnungen $n$. Die Parameter für die attraktive Wechselwirkung und die Gittergeometrie wurden der Auswertung der Beugungsexperimente in Kapitel 4 entnommen, ebenso die zum Vergleich eingezeichneten experimentellen Beugungsintensitäten aus der Messung zu $\theta^{\prime}=21^{\circ}$ und $v^{\prime}=494,9 \mathrm{~ms}^{-1}$. Beide theoretischen Kurven sind um denselben Faktor auf die experimentelle Zählrate skaliert.

und träte auch bei der optischen Beugung auf; der Faktor $\exp \left[-\alpha K\left(\theta_{n}\right)\right]$ hingegen repräsentiert die Asymmetrie aufgrund des bei nicht-senkrechtem Einfall unterschiedlichen Einflusses der attraktiven Wechselwirkung an den Spalträndern. Die übrigen Terme tragen wegen der Funktion $K\left(\theta_{n}\right)$ ebenfalls geringfügig zur Asymmetrie bei. 
In Abbildung 2.5 werden die ursprüngliche Intensitätsformel (2.58) und die durch zwei Kumulanten approximierte Formel (2.75) für charakteristische Parameter gezeigt. Dieser numerische Vergleich rechtfertigt den Abbruch der Kumulantenreihe nach dem zweiten Term im experimentell relevanten Bereich $|n| \lesssim 7$ des Beugungsbildes. Außerdem zeigt Abbildung 2.5 aus einem experimentellen Beugungsbild ermittelte Intensitäten.

\section{Die effektive Spaltbreite der Atombeugung}

Ohne attraktive Wechselwirkung wären sämtliche Kumulanten $R_{j}^{ \pm}$Null und mit ihnen die Terme $\alpha, \Sigma$ und $\Delta$. Die Intensitätsformel (2.75) entspräche dann der bekannten Kirchhoffschen Formel der Beugung an einem Gitter mit der Spaltbreite $S_{0}$ [47, Kap. 8.5]. Im Experiment, also bei vorhandener attraktiver Wechselwirkung, tritt die "effektive Spaltbreite“ $S_{\text {eff }}$ an die Stelle von $S_{0}$, die auf einfache Weise mit der Transmissionsfunktion zusammenhängt: Einsetzen der Formel für die ersten Kumulanten (2.62) in die Definition von (2.66) liefert die Gleichung

$$
S_{\text {eff }}\left(v^{\prime}, \overline{\theta^{\prime}}\right)=S_{0}-\operatorname{Re} \int_{-S_{0} / 2}^{S_{0} / 2} \mathrm{~d} u_{2}\left[1-\tau_{u}^{\mathrm{at}}\left(\overline{\boldsymbol{p}}^{\prime} ; u_{2}\right)\right], \quad v^{\prime}=\frac{\left|\overline{\boldsymbol{p}}^{\prime}\right|}{m} .
$$

Entsprechende Ausdrücke gelten für $\alpha, \Sigma$ und $\Delta$. In Abschnitt A.1.3 wird gezeigt, daß die effektive Spaltbreite in einer einfachen approximativen Relation zur mittleren Geschwindigkeit $v^{\prime}$ der Atome steht (vgl. Gl. A.21):

$$
S_{0}-S_{\text {eff }}\left(v^{\prime}, \overline{\theta^{\prime}}\right) \propto \frac{C_{3}}{\sqrt{v^{\prime}}} .
$$

$C_{3}$ ist eine Konstante, die die Stärke der attraktiven Wechselwirkung beschreibt. Diese Relation wird im Abschnitt 4.2 bei der Auswertung experimenteller AtomBeugungsbilder nützlich sein.

\subsection{Atombeugung bei senkrechtem Einfall}

In den Experimenten von Brühl, Kalinin, Kornilov und Toennies wurden zur Charakterisierung der Geometrie des Transmissionsgitters auch Meßserien bei senkrechtem Einfall aufgenommen. Die notwendige Theorie ist in früheren Arbeiten behandelt worden [3, 18]; aufgrund der speziellen Keilstumpfform der Gitterstege ist sie durch eine geringe Erweiterung auch für Einfallswinkel $0<\theta^{\prime}<\beta$ anwendbar. Der Vollständigkeit halber werden in diesem kurzen Abschnitt die wichtigsten Formeln zusammengefaßt.

\subsubsection{Atombeugung am Gitter bei senkrechtem Einfall}

Das Koordinatensystem in der $\left(x_{1}, x_{2}\right)$-Ebene wird wie in Abb. 2.6 gewählt. Die 


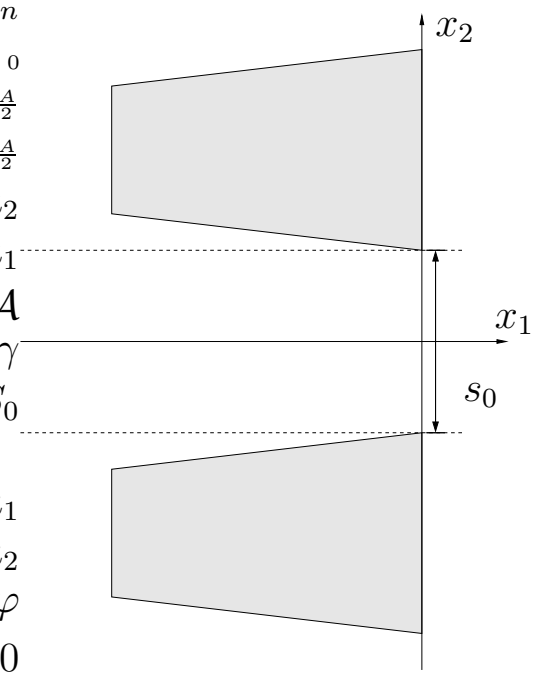

Abbildung 2.6: Spaltbreite $s_{0}$ und Koordinatensystem bei senkrechtem Einfall.

Spaltamplitude des tiefen Einzelspalts der Breite $s_{0}$ wird definiert durch

$$
a_{x}^{\mathrm{at}}\left(\boldsymbol{p}^{\prime} ; \Delta p_{2}\right):=\int_{-\frac{s_{0}}{2}}^{\frac{s_{0}}{2}} \mathrm{~d} x_{2} \exp \left(-\mathrm{i} \Delta p_{2} x_{2} / \hbar\right) \tau_{x}^{\mathrm{at}}\left(\boldsymbol{p}^{\prime} ; x_{2}\right),
$$

wobei $\tau_{x}^{\text {at }}\left(\boldsymbol{p}^{\prime} ; x_{2}\right)$ die Transmissionsfunktion des Spalts bezeichnet. Sie wird explizit in Abschnitt A.1.2 angegeben. Mit Hilfe der Gitterfunktion (2.39) kann die Übergangsamplitude eines im Unendlichen geschlossenen Gitters unter Berücksichtigung der Beugungsbedingung (2.10) geschrieben werden als

$$
t_{\mathrm{git}}^{\mathrm{at}(2)}\left(\boldsymbol{p} ; \boldsymbol{p}^{\prime}\right) \simeq-\frac{\mathrm{i}}{2} \frac{p_{1}+p_{1}^{\prime}}{(2 \pi)^{2} m \hbar}\left[2 \pi \hbar \delta\left(\Delta p_{2}\right)-H_{N}\left(\Delta p_{2}\right) a_{x}^{\mathrm{at}}\left(\boldsymbol{p}^{\prime} ; \Delta p_{2}\right)\right] .
$$

\subsubsection{Das Beugungsbild des Atoms}

Unter entsprechenden Voraussetzungen wie beim nicht-senkrechten Einfall gilt, daß die Beugungsordnungen des Transmissionsgitters bei den Impulsüberträgen $\Delta p_{2}=$ $n 2 \pi \hbar / d$ auftreten und daß ihre Intensitäten durch

$$
I_{n} \propto\left|a_{x}^{\text {at }}\left(\overline{\boldsymbol{p}}^{\prime} ; \Delta p_{2}\right)\right|^{2}
$$

gegeben sind. Die Spaltamplitude (2.78) hat ein zentrales Maximum der Breite $\Delta p_{2} \approx$ $4 \pi \hbar / s_{0}$ um die Vorwärtsrichtung entsprechend $n_{c} \approx d / s_{0}$ darin zu beiden Seiten der Vorwärtsrichtung enthaltener Beugungsordnungen. Die Intensitätsformel (2.80) kann wiederum durch eine Kumulantenentwicklung der Spaltamplitude approximiert werden. Für die experimentell relevanten Beugungsordnungen $n \lesssim 7$ gilt in guter Näherung

$$
I_{n} \propto \mathrm{e}^{-(n 2 \pi \sigma / d)^{2}} \frac{\sin ^{2}\left[n \pi s_{\text {eff }} / d\right]+\sinh ^{2}[n \pi \delta / d]}{(n \pi / d)^{2}}
$$


wobei die Größen $s_{\text {eff }}, \delta$ und $\sigma$ vom Einfallswinkel und den Gitterparametern abhängen [18]. Die „effektive Spaltbreite“ $s_{\text {eff }}$ lautet

$$
s_{\text {eff }}\left(v^{\prime}, \overline{\theta^{\prime}}\right)=s_{0}-\operatorname{Re} \int_{-s_{0} / 2}^{s_{0} / 2} \mathrm{~d} x_{2}\left[1-\tau_{x}^{\mathrm{at}}\left(\overline{\boldsymbol{p}}^{\prime} ; x_{2}\right)\right]
$$

mit der mittleren Geschwindigkeit der Atome $v^{\prime}=\left|\overline{\boldsymbol{p}}^{\prime}\right| / m$ und dem Einfallswinkel $\tan \overline{\theta^{\prime}}=\bar{p}_{2}^{\prime} / \bar{p}_{1}^{\prime}$. Sie gehorcht für große Geschwindigkeiten der in Abschnitt A.1.3 abgeleiteten asymptotischen Relation

$$
s_{0}-s_{\text {eff }}\left(v^{\prime}, \overline{\theta^{\prime}}\right) \propto \frac{C_{3}}{\sqrt{v^{\prime}}} .
$$




\section{Kapitel 3}

\section{Trimerbeugung}

In diesem Kapitel wird die Beugung von Trimeren untersucht. Als Trimere werden hier allgemein schwach gebundene Dreiteilchensysteme bezeichnet. Die drei Teilchen können Atome sein, wie im Fall des Helium-Trimers ${ }^{4} \mathrm{He}_{3}$. Es wird aber nicht ausgeschlossen, daß die Teilchen selbst durch stark gebundene (kovalente) Moleküle realisiert sind. Zum Beispiel ist im „Dreiteilchensystem“ $\mathrm{Ar}_{2}-\mathrm{HCl}$ das Wasserstoff-Atom vergleichsweise stark an das Chlor-Atom gebunden, wohingegen die Bindungen zu den Argon-Atomen schwach sind [48]. Entscheidend wird im folgenden aber sein, daß die Teilchen ihren inneren Zustand bei der Beugung nicht ändern; beispielsweise kann durch eine geringe Teilchengeschwindigkeit im Strahl erreicht werden, daß die Anregungsenergie zum energetisch nächsthöheren Zustand nicht verfügbar ist (eine quantitative Diskussion am Beispiel der Teilchen $\mathrm{H}_{2}$ und $\mathrm{D}_{2}$ wird in Kapitel 5 geführt). Weiterhin wird angenommen, daß sich die Wechselwirkung zwischen den Teilchen als Summe von zentralsymmetrischen Paarpotentialen ausdrücken läßt. Diese Annahme schließt Dreikörperwechselwirkungen aus und stellt somit eine Approximation dar, die aber zumindest im Fall des Helium-Trimers gerechtfertigt ist [34]. Jedes Paarpotential ist im Rahmen der Born-Oppenheimer-Näherung Folge der elektronischen Wechselwirkung des Teilchenpaares; die elektronischen Freiheitsgrade selbst werden im folgenden nicht berücksichtigt.

Der erste Abschnitt dieses Kapitels befaßt sich mit dem freien Trimer. Die darauffolgenden beiden Abschnitte behandeln die streutheoretische Beschreibung der Trimerbeugung. Anschließend wird in Abschnitt 3.4 gezeigt, welche Information über die räumliche Ausdehnung des Trimers aus dem Beugungsbild herausgearbeitet werden kann. Obgleich der Formalismus aufgrund des zusätzlichen Teilchens im Vergleich zur Beugung von Dimeren zu wesentlich umfangreicheren und zum Teil länglichen Ausdrücken führt, erlauben die Ergebnisse doch eine anschauliche und physikalisch verständliche Interpretation.

\subsection{Das freie Trimer}

Zunächst werden einige Aspekte des freien Trimers, also in Abwesenheit äußerer Wechselwirkungen, untersucht. Nach der Definition geeigneter Koordinaten wird der Hamilton-Operator angegeben und einige später in diesem Kapitel benötigte Rela- 
tionen für die Bindungszustände des Trimers abgeleitet. Im Hinblick auf das HeliumTrimer wird anschließend die Symmetrie der Bindungszustände für den Fall spinloser, ununterscheidbarer Teilchen behandelt. Schließlich werden Ergebnisse aus der theoretischen Literatur für die Bindungsenergie und den mittleren Paarabstand der Bindungszustände des Helium-Trimers ${ }^{4} \mathrm{He}_{3}$ zitiert. Eine vertiefende Behandlung der Bindungszustände findet sich in Anhang B.

\subsubsection{Unterscheidbare Teilchen}

Die Massen der drei Teilchen des Trimers, werden mit $m_{i}$ bezeichnet, ihre Orte mit $\mathbf{r}_{i}$ und die konjugierten Impulse mit $\mathbf{p}_{i}$. Die Wechselwirkung jedes der drei Teilchenpaare $(j k)$ wird durch ein Potential $v_{j k}\left(\left|\mathbf{r}^{(j k)}\right|\right)$ beschrieben, das nur vom Betrag der Relativkoordinate $\mathbf{r}^{(j k)}:=\mathbf{r}_{j}-\mathbf{r}_{k}$ des Paares abhängt. Zwar kann die Anwesenheit des jeweils dritten Teilchens die Paarwechselwirkung stören: Bei van der WaalsClustern, wie dem Helium-Trimer, verzerrt das dritte Teilchen die Polarisierung der anderen beiden. Dieser Effekt wird als Dreikörperwechselwirkung bezeichnet und äußert sich in einem zusätzlichen Potentialterm $V_{123}\left(\mathbf{r}_{1}, \mathbf{r}_{2}, \mathbf{r}_{3}\right)$. Speziell im HeliumTrimer aber ist der Einfluß der Dreikörperwechselwirkung laut quantenchemischer Rechnungen sehr gering [34]. Im folgenden wird verallgemeinernd angenommen, daß auch für andere schwach gebundene Trimere durch die Vernachlässigung der Dreikörperwechselwirkung keine wesentliche quantitative Ungenauigkeit eingeführt wird. Der Hamilton-Operator des freien Trimers lautet in diesem Modell

$$
H=\sum_{i=1}^{3} \frac{\hat{\mathbf{p}}_{i}^{2}}{2 m_{i}}+v_{12}\left(\left|\hat{\mathbf{r}}^{(12)}\right|\right)+v_{23}\left(\left|\hat{\mathbf{r}}^{(23)}\right|\right)+v_{31}\left(\left|\hat{\mathbf{r}}^{(31)}\right|\right) .
$$

In Abwesenheit äußerer Kräfte ist der Schwerpunktimpuls eine Erhaltungsgröße. Ein angepaßtes Koordinatensystem aus Schwerpunkt- und Relativkoordinaten bieten die sogenannten Jacobi-Koordinaten $\mathbf{R}, \boldsymbol{\rho}, \mathbf{r}$ (vgl. Abb. 3.1), von denen es drei äquivalente Realisierungen gibt. Ihre Definition läßt sich in Blockmatrixform notieren,

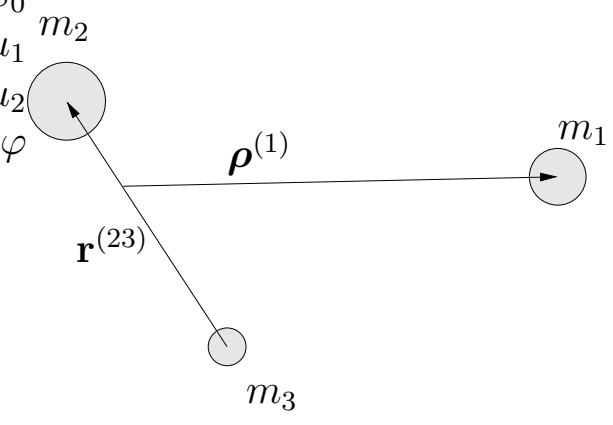

\footnotetext{
Abbildung 3.1: Eine von drei Realisierungen der Jacobi-Koordinaten zur Beschreibung der Relativbewegung des Trimers. Anschaulich gesprochen zeigt der Vektor $\boldsymbol{\rho}^{(1)}$ vom Schwerpunkt des Paares (23) zum Teilchen 1, während $\mathbf{r}^{(23)}$ von Teilchen 3 zu Teilchen 2 zeigt. Die dritte, nicht eingezeichnete Jacobi-Koordinate ist der Schwerpunktvektor $\mathbf{R}$, der von der Realisierung unabhängig ist.
} 


$$
\left(\begin{array}{l}
\mathbf{R} \\
\boldsymbol{\rho}^{(i)} \\
\mathbf{r}^{(j k)}
\end{array}\right):=\left(\begin{array}{rrr}
\frac{m_{i}}{M} & \frac{m_{j}}{M} \mathbb{1} & \frac{m_{k}}{M} \mathbb{1} \\
\mathbb{1} & -\frac{m_{j}}{m_{j}+m_{k}} \mathbb{1} & -\frac{m_{k}}{m_{j}+m_{k}} \mathbb{1} \\
\mathbb{0} & \mathbb{1} & -\mathbb{1}
\end{array}\right)\left(\begin{array}{l}
\mathbf{r}_{i} \\
\mathbf{r}_{j} \\
\mathbf{r}_{k}
\end{array}\right)
$$

wobei $\mathbb{0}$ und $\mathbb{1}$ jeweils $3 \times 3$-Matrizen darstellen, und $M:=m_{1}+m_{2}+m_{3}$ die Gesamtmasse des Trimers bezeichnet. Die Anordnung der Indizes wird auf die zyklischen Vertauschungen

$$
(i j k)=(123),(231),(312)
$$

eingeschränkt. Dadurch ist unter anderem gewährleistet, daß die Jacobi-Determinante der Transformation (3.2) den Wert +1 besitzt. Aus Gleichung (3.2) läßt sich auch die Transformation zwischen den verschiedenen Realisierungen der Jacobi-Koordinaten untereinander herleiten. Die Relativkoordinaten transformieren sich nach der Gleichung

$$
\left(\begin{array}{l}
\boldsymbol{\rho}^{(j)} \\
\mathbf{r}^{(k i)}
\end{array}\right)=\mathbb{d}^{(j i)}\left(\begin{array}{l}
\boldsymbol{\rho}^{(i)} \\
\mathbf{r}^{(j k)}
\end{array}\right)
$$

mit der $6 \times 6$-Matrix

$$
\mathbb{d}^{(j i)}:=\left(\begin{array}{rr}
-\frac{m_{i}}{m_{i}+m_{k}} \mathbb{1} & \frac{m_{k} M}{\left(m_{j}+m_{k}\right)\left(m_{k}+m_{j}\right)} \mathbb{1} \\
-\mathbb{1} & -\frac{m_{j}}{m_{j}+m_{k}} \mathbb{1}
\end{array}\right) .
$$

Die Schwerpunktkoordinate $\mathbf{R}$ ist in allen drei Realisierungen der Jacobi-Koordinaten identisch. Faßt man beides zusammen zu der symbolischen Blockdiagonalmatrix

$$
\mathbb{D}^{(j i)}:=\left(\begin{array}{cc}
\mathbb{1} & 0 \\
0 & \mathbb{d}^{(j i)}
\end{array}\right),
$$

so kann die gesamte Transformation geschrieben werden als

$$
\left(\begin{array}{l}
\mathbf{R} \\
\boldsymbol{\rho}^{(j)} \\
\mathbf{r}^{(k i)}
\end{array}\right)=\mathbb{D}^{(j i)}\left(\begin{array}{l}
\mathbf{R} \\
\boldsymbol{\rho}^{(i)} \\
\mathbf{r}^{(j k)}
\end{array}\right)
$$

Die drei Matrizen $\mathbb{D}^{(j i)}$ sind invertierbar und erfüllen die Relationen

$$
\begin{aligned}
\mathbb{D}^{(j i)} \mathbb{D}^{(i k)} \mathbb{D}^{(k j)} & =\mathbb{1} \\
\operatorname{det} \mathbb{D}^{(i j)} & =1
\end{aligned}
$$

Die Gleichungen (3.8) und (3.9) gelten analog für die drei Matrizen $\mathbb{d}^{(j i)}$.

Die zu den Jacobi-Koordinaten $\mathbf{R}, \boldsymbol{\rho}^{(i)}, \mathbf{r}^{(j k)}$ konjugierten Schwerpunkt- und Relativimpulse $\mathbf{P}, \mathbf{q}^{(i)}, \mathbf{p}^{(j k)}$ können nach der üblichen Vorschrift aus der klassischen Lagrange-Funktion des Trimers berechnet werden. Sie stehen mit den Teilchenimpulsen $\mathbf{p}_{1}, \mathbf{p}_{2}, \mathbf{p}_{3}$ durch die Gleichung

$$
\left(\begin{array}{l}
\mathbf{P} \\
\mathbf{q}^{(i)} \\
\mathbf{p}^{(j k)}
\end{array}\right)=\left(\begin{array}{rrrr} 
& \mathbb{1} & \mathbb{1} & \mathbb{1} \\
\frac{m_{j}+m_{k}}{M} \mathbb{1} & -\frac{m_{i}}{M} & -\frac{m_{i}}{M} \\
& \mathbb{1} & \frac{m_{k}}{m_{j}+m_{k}} \mathbb{1} & -\frac{m_{j}}{m_{j}+m_{k}} \mathbb{1}
\end{array}\right)\left(\begin{array}{l}
\mathbf{p}_{i} \\
\mathbf{p}_{j} \\
\mathbf{p}_{k}
\end{array}\right)
$$


in Verbindung. Eine kurze Rechnung zeigt darüberhinaus, daß die Impulse verschiedener Realisierungen der Jacobi-Koordinaten untereinander mit dem transponierten Inversen der Matrix $\mathbb{D}^{(j i)}$ transformiert werden.

$$
\left(\begin{array}{l}
\mathbf{P} \\
\mathbf{q}^{(j)} \\
\mathbf{p}^{(k i)}
\end{array}\right)=\left[\mathbb{D}^{(j i)-1}\right]^{T}\left(\begin{array}{l}
\mathbf{P} \\
\mathbf{q}^{(i)} \\
\mathbf{p}^{(j k)}
\end{array}\right)
$$

Das symbolische Skalarprodukt

$$
\left(\begin{array}{l}
\mathbf{P} \\
\mathbf{q}^{(j)} \\
\mathbf{p}^{(k i)}
\end{array}\right) \cdot\left(\begin{array}{l}
\mathbf{R} \\
\boldsymbol{\rho}^{(j)} \\
\mathbf{r}^{(k i)}
\end{array}\right)=\left(\begin{array}{l}
\mathbf{P} \\
\mathbf{q}^{(i)} \\
\mathbf{p}^{(j k)}
\end{array}\right) \cdot\left(\begin{array}{l}
\mathbf{R} \\
\boldsymbol{\rho}^{(i)} \\
\mathbf{r}^{(j k)}
\end{array}\right)
$$

ist somit unabhängig von der Realisierung der Jacobi-Koordinaten. Ausgedrückt in der Realisierung $\boldsymbol{\rho}^{(i)}, \mathbf{r}^{(j k)}$ setzt sich der Hamilton-Operator $H=H_{0}+V$ aus der gesamten kinetischen Energie

$$
H_{0}:=\frac{1}{2 M} \hat{\mathbf{P}}^{2}+\frac{M}{2 m_{i}\left(m_{j}+m_{k}\right)} \hat{\mathbf{q}}^{(i) 2}+\frac{m_{j}+m_{k}}{2 m_{j} m_{k}} \hat{\mathbf{p}}^{(j k) 2}
$$

und der potentiellen Energie

$$
V:=v_{i j}\left(\left|\hat{\boldsymbol{\rho}}^{(i)}-\frac{m_{k}}{m_{j}+m_{k}} \hat{\mathbf{r}}^{(j k)}\right|\right)+v_{j k}\left(\left|\hat{\mathbf{r}}^{(j k)}\right|\right)+v_{k i}\left(\left|\hat{\boldsymbol{\rho}}^{(i)}+\frac{m_{j}}{m_{j}+m_{k}} \hat{\mathbf{r}}^{(j k)}\right|\right)
$$

zusammen. Der zugrundeliegende Hilbertraum $\mathcal{H}$ faktorisiert in das Produkt $\mathcal{H}_{\mathrm{CM}} \otimes$ $\mathcal{H}_{\mathrm{REL}}$, wobei $\mathcal{H}_{\mathrm{CM}}$ durch die uneigentlichen Eigenzustände $|\mathbf{P}\rangle$ des Schwerpunktimpulses aufgespannt wird, und $\mathcal{H}_{\text {REL }}$ durch die Eigenzustände des Relativanteils. Gebundene Zustände aus $\mathcal{H}_{\text {REL }}$ zur negativen Bindungsenergie $E_{\gamma}$ werden mit $\left|\phi_{\gamma}\right\rangle$ bezeichnet. Sie erfüllen die zeitunabhängige Schrödinger-Gleichung

$$
H\left|\mathbf{P}, \phi_{\gamma}\right\rangle=E\left|\mathbf{P}, \phi_{\gamma}\right\rangle \quad \text { mit } \quad\left|\mathbf{P}, \phi_{\gamma}\right\rangle \equiv|\mathbf{P}\rangle_{\mathrm{CM}} \otimes\left|\phi_{\gamma}\right\rangle_{\mathrm{REL}}
$$

zu dem Energieeigenwert

$$
E:=\frac{|\mathbf{P}|^{2}}{2 M}+E_{\gamma}
$$

Streuzustände des Relativanteils des Hamilton-Operators werden im folgenden nicht betrachtet.

\section{Impulsraum- und Ortsraumdarstellungen der Bindungszustände}

Die in $\mathcal{H}_{\mathrm{REL}}$ liegenden Eigenzustände der Relativkoordinaten-Operatoren $\hat{\boldsymbol{\rho}}^{(i)}$ und $\hat{\mathbf{r}}^{(j k)}$ werden mit $|\boldsymbol{\rho}, \mathbf{r}\rangle_{i, j k} \equiv|\boldsymbol{\rho}\rangle_{i} \otimes|\mathbf{r}\rangle_{j k}$ bezeichnet. Sie sind bis auf globale Phasenfaktoren durch die Eigenwertgleichungen

$$
\hat{\boldsymbol{\rho}}^{(i)}|\boldsymbol{\rho}, \mathbf{r}\rangle_{i, j k}=\boldsymbol{\rho}|\boldsymbol{\rho}, \mathbf{r}\rangle_{i, j k} \quad \text { und } \quad \hat{\mathbf{r}}^{(j k)}|\boldsymbol{\rho}, \mathbf{r}\rangle_{i, j k}=\mathbf{r}|\boldsymbol{\rho}, \mathbf{r}\rangle_{i, j k}
$$


und die Normierung

$$
{ }_{i, j k}\left\langle\boldsymbol{\rho}^{\prime}, \mathbf{r}^{\prime} \mid \boldsymbol{\rho}, \mathbf{r}\right\rangle_{i, j k}=\delta^{(3)}\left(\boldsymbol{\rho}-\boldsymbol{\rho}^{\prime}\right) \delta^{(3)}\left(\mathbf{r}-\mathbf{r}^{\prime}\right)
$$

bestimmt. Zwischen den Eigenzuständen von Relativkoordinaten-Operatoren verschiedener Realisierungen bestehen Beziehungen, die im weiteren Verlauf dieses Kapitels von Interesse sind. Beispielsweise zeigt die Anwendung der auch für Operatoren gültigen Transformation $\mathbb{d}^{(21)}$ (3.5) auf den Zustand $|\boldsymbol{\rho}, \mathbf{r}\rangle_{1,23}$, daß dieser proportional zum Eigenzustand $\left|\mathbb{d}^{(21)}\left(\begin{array}{c}\boldsymbol{\rho} \\ \mathbf{r}\end{array}\right)\right\rangle_{2,31}$ der Operatoren $\hat{\boldsymbol{\rho}}^{(2)}$ und $\hat{\mathbf{r}}^{(31)}$ ist $^{1}$. Wegen $\operatorname{det} \mathbb{d}^{(21)}=1$ ist die Proportionalitätskonstante eine reine Phase, die festgelegt wird durch die Forderung

$$
\left|\mathbb{d}^{(21)}\left(\begin{array}{l}
\boldsymbol{\rho} \\
\mathbf{r}
\end{array}\right)\right\rangle_{2,31}=|\boldsymbol{\rho}, \mathbf{r}\rangle_{1,23} .
$$

Entsprechend wird die Phase des verbleibenden Eigenzustandes vorgegeben durch

$$
\left|\mathbb{d}^{(32)} \mathbb{d}^{(21)}\left(\begin{array}{c}
\boldsymbol{\rho} \\
\mathbf{r}
\end{array}\right)\right\rangle_{3,12}=|\boldsymbol{\rho}, \mathbf{r}\rangle_{1,23} .
$$

Es ist instruktiv, für die Eigenwerte in (3.19) und (3.20) die spezielle Realisierung $\boldsymbol{\rho}^{(1)}, \mathbf{r}^{(23)}$ einzusetzen. Mit Hilfe der Transformation (3.5) folgen aus (3.19) und (3.20) sofort die einfachen Beziehungen

$$
\left|\boldsymbol{\rho}^{(1)}, \mathbf{r}^{(23)}\right\rangle_{1,23}=\left|\boldsymbol{\rho}^{(2)}, \mathbf{r}^{(31)}\right\rangle_{2,31}=\left|\boldsymbol{\rho}^{(3)}, \mathbf{r}^{(12)}\right\rangle_{3,12}
$$

Entsprechend folgt für die Projektionen eines beliebigen Zustandes $|\psi\rangle \in \mathcal{H}_{\text {REL }}$ auf die Eigenvektoren verschiedener Realisierungen die Formel

$$
\begin{aligned}
\psi^{(i, j k)}\left(\boldsymbol{\rho}^{(i)}, \mathbf{r}^{(j k)}\right) & \equiv{ }_{i, j k}\left\langle\boldsymbol{\rho}^{(i)}, \mathbf{r}^{(j k)} \mid \psi\right\rangle \\
& ={ }_{j, k i}\left\langle\boldsymbol{\rho}^{(j)}, \mathbf{r}^{(k i)} \mid \psi\right\rangle \equiv \psi^{(j, k i)}\left(\boldsymbol{\rho}^{(j)}, \mathbf{r}^{(k i)}\right) .
\end{aligned}
$$

Die Koordinatentransformation $\mathbb{D}^{(j i)}$ kann auch durch eine unitäre Transformation

$$
\mathcal{U}\left(\mathbb{D}^{(j i)-1}\right):=(1)_{\mathrm{CM}} \otimes\left(\mathcal{U}\left(\mathbb{d}^{(j i)-1}\right)\right)_{\mathrm{REL}}
$$

auf dem Hilbertraum $\mathcal{H}$ dargestellt werden, die hier durch ihre Wirkung auf das vollständige System uneigentlicher Eigenzustände $|\mathbf{P}, \boldsymbol{\rho}, \mathbf{r}\rangle_{j, k i}$ definiert wird:

$$
\mathcal{U}^{\dagger}\left(\mathbb{D}^{(j i)-1}\right)|\mathbf{P}, \boldsymbol{\rho}, \mathbf{r}\rangle_{j, k i}=|\mathbf{P}\rangle \otimes \mathcal{U}^{\dagger}\left(\mathbb{d}^{(j i)-1}\right)|\boldsymbol{\rho}, \mathbf{r}\rangle_{j, k i}:=\left|\mathbf{P}, \mathbb{d}^{(j i)}\left(\begin{array}{l}
\boldsymbol{\rho} \\
\mathbf{r}
\end{array}\right)\right\rangle_{j, k i}
$$

Eine kurze Rechnung zeigt, daß die unitäre Transformation auf $\mathcal{H}_{\text {REL }}$ die Ortsraumdarstellung eines Zustandes $|\psi\rangle$ in der üblichen Weise verdreht:

$$
\left(\mathcal{U}\left(\mathbb{d}^{(j i)-1}\right) \psi\right)^{(j, k i)}(\boldsymbol{\rho}, \mathbf{r})=\psi^{(j, k i)}\left(\mathbb{d}^{(j i)}\left(\begin{array}{l}
\boldsymbol{\rho} \\
\mathbf{r}
\end{array}\right)\right) .
$$

\footnotetext{
${ }^{1}$ Die Schreibweise $\left|\mathbb{d}^{(21)}\left(\begin{array}{l}\boldsymbol{\rho} \\ \mathbf{r}\end{array}\right)\right\rangle_{2,31}$ steht abkürzend für $|\widetilde{\boldsymbol{\rho}}, \widetilde{\mathbf{r}}\rangle_{2,31}$ wobei $\left(\begin{array}{l}\widetilde{\boldsymbol{\rho}} \\ \widetilde{\mathbf{r}}\end{array}\right)=\mathbb{d}^{(21)}\left(\begin{array}{l}\boldsymbol{\rho} \\ \mathbf{r}\end{array}\right)$ etc.
} 


\subsubsection{Spinlose, ununterscheidbare Teilchen}

Sind die drei Teilchen im Trimer spinlos und ununterscheidbar, wie es im HeliumTrimer ${ }^{4} \mathrm{He}_{3}$ der Fall ist, so nimmt der Hamilton-Operator unabhängig von der Wahl der Jacobi-Koordinaten immer dieselbe Gestalt an. Mit den Bezeichnungen $m=m_{i}$ und $v=v_{j k}$ lautet er

$$
H=\frac{1}{2 M} \hat{\mathbf{P}}^{2}+\frac{3}{4 m} \hat{\mathbf{q}}^{2}+\frac{1}{m} \hat{\mathbf{p}}^{2}+v\left(\left|\hat{\boldsymbol{\rho}}-\frac{1}{2} \hat{\mathbf{r}}\right|\right)+v(|\hat{\mathbf{r}}|)+v\left(\left|\hat{\boldsymbol{\rho}}+\frac{1}{2} \hat{\mathbf{r}}\right|\right) .
$$

Die drei Transformationsmatrizen $\mathbb{D}^{(j i)}$ der Relativkoordinaten (3.6) werden identisch. Man findet

$$
\mathbb{D}:=\left(\begin{array}{ll}
\mathbb{1} & 0 \\
0 & \mathbb{d}
\end{array}\right) \quad \text { mit } \quad \mathbb{d}:=\left(\begin{array}{rr}
-\frac{1}{2} \mathbb{1} & \frac{3}{4} \mathbb{1} \\
-\mathbb{1} & -\frac{1}{2} \mathbb{1}
\end{array}\right)
$$

und der Eigenschaft

$$
\mathbb{D}^{3}=\mathbb{1}
$$

Wiederholte Anwendung von $\mathbb{d}$ erzeugt also aus einer anfänglichen Realisierung $\boldsymbol{\rho}, \mathbf{r}$ der Relativkoordinaten zunächst die beiden anderen Realisierungen und anschließend wieder die anfängliche. Die kanonisch konjugierten Relativimpulse sind nach Gleichung (3.11) mit dem transponierten Inversen von $\mathbb{d}$ zu transformieren. Aus (3.27) folgt unmittelbar

$$
\left[\mathbb{D}^{-1}\right]^{T}:=\left(\begin{array}{cc}
\mathbb{1} & 0 \\
0 & {\left[\mathbb{d}^{-1}\right]^{T}}
\end{array}\right) \quad \text { mit } \quad\left[\mathbb{d}^{-1}\right]^{T}:=\left(\begin{array}{cc}
-\frac{1}{2} \mathbb{1} & \mathbb{1} \\
-\frac{3}{4} \mathbb{1} & -\frac{1}{2} \mathbb{1}
\end{array}\right) .
$$

Die Eigenzustände der Relativkoordinaten-Operatoren $\hat{\boldsymbol{\rho}}$ und $\hat{\mathbf{r}}$ zu den Eigenwerten $\boldsymbol{\rho}$ beziehungsweise $\mathbf{r}$ werden im folgenden mit $|\boldsymbol{\rho}, \mathbf{r}\rangle$ bezeichnet, und entsprechend für die Eigenzustände $|\mathbf{q}, \mathbf{p}\rangle$ der Relativimpuls-Operatoren $\hat{\mathbf{q}}$ und $\hat{\mathbf{p}}$. Die Projektion eines beliebigen Zustandes $|\psi\rangle \in \mathcal{H}_{\text {REL }}$ auf die Relativkoordinaten wird mit $\psi(\boldsymbol{\rho}, \mathbf{r}) \equiv$ $\langle\boldsymbol{\rho}, \mathbf{r} \mid \psi\rangle$ bezeichnet. Definiert man unitäre Transformationen $\mathcal{U}\left(\mathbb{D}^{-1}\right)$ und $\mathcal{U}\left(\mathbb{d}^{-1}\right)$ analog zu den Gleichungen (3.23) und (3.24), so transformiert sich die Projektion entsprechend Gleichung (3.25) wie

$$
\mathcal{U}\left(\mathbb{d}^{-1}\right) \psi(\boldsymbol{\rho}, \mathbf{r})=\psi\left(\mathbb{d}\left(\begin{array}{l}
\boldsymbol{\rho} \\
\mathbf{r}
\end{array}\right)\right) .
$$

Der Hamilton-Operator $H$ (3.26) ist invariant unter der Koordinatentransformation $\mathbb{D}$ (3.27), die auch als starre Rotation aller drei Teilchen auf die Plätze ihrer Nachbarn interpretiert werden kann. Invarianz unter $\mathbb{D}$ bedeutet, daß $H$ und $\mathcal{U}\left(\mathbb{D}^{-1}\right)$ gemeinsam diagonalisiert werden können. Die Eigenwerte von $\mathcal{U}\left(\mathbb{D}^{-1}\right)$ lauten +1 und $\exp ( \pm \mathrm{i} 2 \pi / 3)$, wie bereits an der Eigenschaft (3.28) zu erkennen ist. Da Wellenfunktionen von spinlosen, ununterscheidbaren Teilchen unter Teilchenaustausch aber symmetrisch sein müssen, können nur solche zum Eigenwert +1 physikalische Bedeutung haben. Ist $\varphi(\boldsymbol{\rho}, \mathbf{r})$ eine beliebige (unsymmetrisierte) Eigenfunktion von $H$, so wird die korrekte Symmetrie unter der Operation $\mathcal{U}\left(\mathbb{D}^{-1}\right)$ per Konstruktion durch Bildung der Summe

$$
\phi(\boldsymbol{\rho}, \mathbf{r}):=\frac{1}{N}\left\{\varphi(\boldsymbol{\rho}, \mathbf{r})+\mathcal{U}\left(\mathbb{d}^{-1}\right) \varphi(\boldsymbol{\rho}, \mathbf{r})+\mathcal{U}\left(\mathbb{d}^{-1}\right)^{2} \varphi(\boldsymbol{\rho}, \mathbf{r})\right\}
$$


gewährleistet, wobei $N$ eine Normierungskonstante ist. Die Funktion $\phi$ ist wieder eine Eigenfunktion von $H$.

Weiterhin vertauscht $H$ mit den Paritätsoperatoren $\Pi_{\mathbf{r}}$ und $\Pi_{\boldsymbol{\rho}}$, die das Vorzeichen der Relativkoordinaten invertieren. Zwar kommutieren die Paritätsoperatoren nicht auf dem gesamten Hilbertraum mit $\mathcal{U}\left(\mathbb{D}^{-1}\right)$; allerdings verschwinden aber die Kommutatoren auf dem Unterraum von $\mathcal{H}_{\mathrm{REL}}$, der durch die nach der Vorschrift (3.31) symmetrisierten, physikalisch relevanten Funktionen $\phi$ aufgespannt wird.

$$
\begin{aligned}
& {\left[\Pi_{\mathbf{r}}, \mathcal{U}\left(\mathbb{D}^{-1}\right)\right] \phi=0} \\
& {\left[\Pi_{\boldsymbol{\rho}}, \mathcal{U}\left(\mathbb{D}^{-1}\right)\right] \phi=0}
\end{aligned}
$$

Die Funktionen $\phi$ können also zusätzlich zu fester Parität in $\mathbf{r}$ und $\boldsymbol{\rho}$ gewählt werden. Die Inversion der Koordinate $\mathbf{r}$ entspricht aber dem Austausch zweier Teilchen; für spinlose, ununterscheidbare Teilchen sind daher nur Eigenfunktionen zur Parität +1 in $\mathbf{r}$ zulässig. Die Inversion von $\boldsymbol{\rho}$ ist nicht als Teilchenaustausch interpretierbar, so daß hier zunächst beide Paritäten erlaubt sind; allerdings ist speziell bei TrimerEigenfunktionen mit Gesamtdrehimpuls $L=0$ nur die Parität +1 physikalisch realisiert, wie eine Analyse in Anhang B zeigt (vgl. S. 122). Das Betragsquadrat der so gewählten physikalischen Bindungswellenfunktion erfüllt also die Relationen

$$
|\phi(\boldsymbol{\rho}, \mathbf{r})|^{2}=|\phi(\boldsymbol{\rho},-\mathbf{r})|^{2}=|\phi(-\boldsymbol{\rho}, \mathbf{r})|^{2}=|\phi(-\boldsymbol{\rho},-\mathbf{r})|^{2} .
$$

\subsubsection{Das Helium-Trimer ${ }^{4} \mathrm{He}_{3}$}

Seit den siebziger Jahren des zwanzigsten Jahrhunderts wurden die Bindungszustände und Bindungsenergien des Helium-Trimers ${ }^{4} \mathrm{He}_{3}$ in einer beträchtlichen Anzahl von Arbeiten untersucht; aktuelle Veröffentlichungen bezeugen, daß die Forschung auf diesem Gebiet noch keineswegs abgeschlossen ist. Besondere Aufmerksamkeit wurde der vermuteten Existenz eines sogenannten „Efimov-Zustandes“ im ${ }^{4} \mathrm{He}_{3}$ zuteil, der bereits 1977 mit Hilfe von numerischen Rechnungen und verschiedenen damals verfügbaren Helium-Helium-Paarpotentialen vorhergesagt wurde [21, 22]. Als Efimov-Effekt wird eine scheinbar widersprüchliche theoretische Beobachtung bezeichnet, die in gewissen Dreiteilchensystemen auftritt: ist die effektive Reichweite der Paarwechselwirkung wesentlich kleiner als die Zweiteilchen-Streulänge, so hätte die hypothetische Abschwächung der Paarwechselwirkung die Einführung bis zu unendlich vieler quantenmechanischer Bindungszustände, sogenannter Efimov-Zustände, zur Folge [38].

In frühen Arbeiten wurden die sogenannten Faddeev-Gleichungen [49], die die praktische Behandlung quantenmechanischer Dreiteilchensysteme ermöglichen, numerisch näherungsweise im Impulsraum gelöst. Genauere Rechnungen folgten, die zum Teil ebenfalls auf den Faddeev-Gleichungen im Impulsraum [23] oder im Ortsraum [24, 25, 26, 27, 28, 29] basierten, sowie Rechnungen, die die Schrödinger-Gleichung in sogenannten adiabatischen hypersphärischen Koordinaten lösten [30]. Darüberhinaus gibt es verschiedene Variationsrechnungen für das Helium-Trimer [31, 32] und sogenannte „diffusion quantum Monte Carlo" Rechnungen [33]. Sie werden komplementiert durch quantenchemische „ab initio“-Rechnungen, die das Vielteilchensy- 
stem aus Elektronen und Kernen betrachten [34]. Diese quantenchemischen Rechnungen liegen auch den Berechnungen der Helium-Helium-Paarpotentiale zugrunde, für welche sich in Ref. [50] eine aktuelle Übersicht findet. Schließlich gibt es jüngere theoretische Arbeiten zu universellen Skalierungseigenschaften der Bindungszustände $[35,36,37]$.

Die meisten Veröffentlichungen stimmen in der Aussage überein, daß das ${ }^{4} \mathrm{He}_{3}$ neben dem Grundzustand nur einen angeregten Zustand besitzt, der ein EfimovZustand ist. Beide Zustände gehören zum Gesamtdrehimpuls $L=0$. Vor kurzem wurde zwar von González-Lezana et al sowohl die Efimov-Artigkeit des angeregten Zustands bezweifelt [51] als auch ein Zustand zu $L=1$ vorhergesagt [52]. Diesen Arbeiten wurde allerdings durch Lee et al widersprochen [53].

In Tabelle 3.1 werden die Bindungsenergien $E_{\gamma}$, die mittleren Paarabstände $\langle r\rangle$ und deren Streuung $\sqrt{\left\langle r^{2}\right\rangle}$ für die Bindungszustände des Helium-Trimers, die mit dem sogenannten TTY-Potential [54] berechnet wurden, aus Ref. [32] reproduziert. Der Grundzustand des Helium-Trimers ist demzufolge circa fünfzigfach stärker ge-

\begin{tabular}{|cc|cc|}
\hline & & Grundzustand & Angeregter Zustand \\
\hline \hline$E_{\gamma}$ & {$[\mathrm{mK}]$} & $-126,4$ & $-2,277$ \\
$E_{\gamma}$ & {$[\mu \mathrm{eV}]$} & $-10,89$ & $-0,1962$ \\
\hline$\langle r\rangle$ & {$[\AA]$} & 9,610 & 79,75 \\
$\sqrt{\left\langle r^{2}\right\rangle}$ & {$[\AA]$} & 10,96 & 97,21 \\
\hline
\end{tabular}

Tabelle 3.1: Bindungsenergien $E_{\gamma}$, mittlere Paarabstände $\langle r\rangle$ und deren Streuung $\sqrt{\left\langle r^{2}\right\rangle}$ im Helium-Trimer ${ }^{4} \mathrm{He}_{3}$ mit TTY-Potential nach Ref. [32]. Darin bezeichnet $r$ den Betrag der Jacobi-Koordinate $\mathbf{r}$.

bunden als der angeregte Zustand und räumlich wesentlich kompakter. Der angeregte Zustand ist in etwa so stark gebunden und räumlich ausgedehnt wie das Helium-Di$\operatorname{mer}\left(E_{\mathrm{g}}=-0,1 \mu \mathrm{eV} ;\langle r\rangle=52 \AA[5]\right)$.

Da die Bindungswellenfunktionen im allgemeinen von den sechs Komponenten der Koordinaten $\boldsymbol{\rho}$ und $\mathbf{r}$ abhängen können, sind sie nur schwer zu visualisieren. Eine Möglichkeit ist die Darstellung der sogenannten hyperradialen Aufenthaltwahrscheinlichkeitsdichte $P(R)$ : Dazu werden die Beträge der Jacobi-Koordinaten $\rho:=|\boldsymbol{\rho}|$ und $r:=|\mathbf{r}|$ ersetzt durch die hypersphärischen Koordinaten ${ }^{2}$

$$
\begin{aligned}
\mu_{0} R^{2} & :=\mu_{1} r^{2}+\mu_{2} \rho^{2} \\
\tan \phi & :=\sqrt{\frac{\mu_{2}}{\mu_{1}}} \frac{\rho}{r},
\end{aligned}
$$

wobei $\mu_{0}$ eine frei wählbare Konstante mit der Einheit Masse [30] ist. Die „Massen“ $\mu_{1}$ und $\mu_{2}$ sind definiert durch

$$
\mu_{1}:=\frac{1}{2} m, \quad \mu_{2}:=\frac{2}{3} m
$$

${ }^{2}$ Die hypersphärische Koordinate $R$ ist nicht zu verwechseln mit der Schwerpunktkoordinate $\mathbf{R}$, die hier nicht auftritt. 
und haben die Bedeutung von reduzierten Massen im Trimer. Die hypersphärische Aufenthaltswahrscheinlichkeitsdichte $P(R)$ der Bindungswellenfunktion $\phi(\boldsymbol{\rho}, \mathbf{r})$ wird definiert als

$$
P\left(R^{\prime}\right):=\int \mathrm{d}^{3} \rho \int \mathrm{d}^{3} r \delta\left(R(\rho, r)-R^{\prime}\right)|\phi(\boldsymbol{\rho}, \mathbf{r})|^{2},
$$

wobei für $R(\rho, r)$ die Definition (3.34a) einzusetzen ist. Diese eindimensionale Wahrscheinlichkeitsdichte kann grafisch dargestellt werden. Abbildung 3.2 zeigt $P(R)$ für die beiden Zustände des Helium-Trimers auf der Grundlage der nach Anhang B für die Zwecke der vorliegenden Arbeit numerisch berechneten Trimer-Bindungswellenfunktionen. Der Erwartungswert der hypersphärischen Koordinate $\langle R\rangle$ ist ein Maß

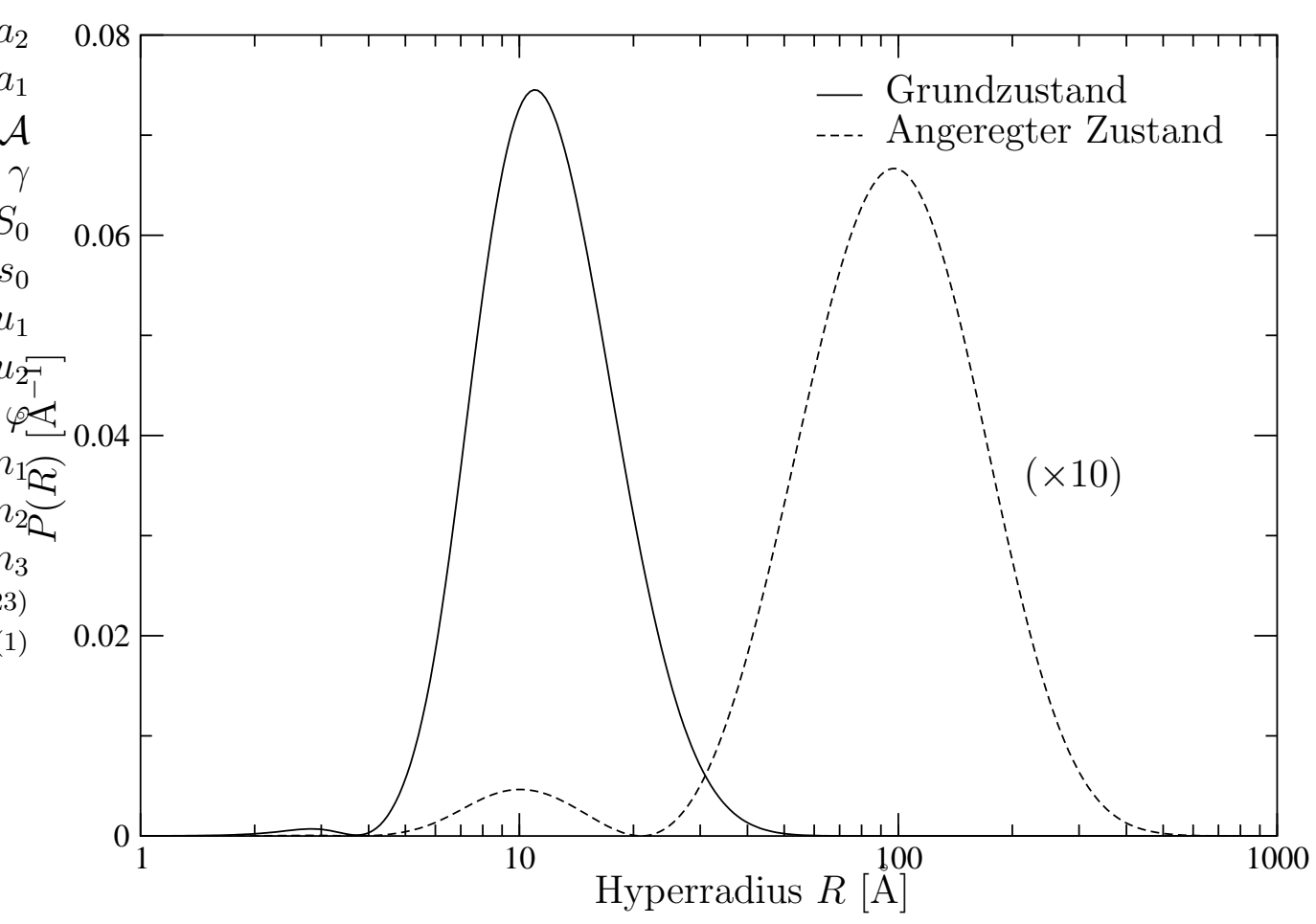

Abbildung 3.2: Halblogarithmische Auftragung der hyperradialen Aufenthaltswahrscheinlichkeitsdichten $P(R)$ der beiden Bindungszustände des Helium-Trimers. Die freie Konstante $\mu_{0}$ wurde für die Grafik zu $\mu_{0} / m=1 / 2$ gewählt.

für die räumliche Ausdehnung des Trimers. Es ist allerdings zu beachten, daß er von der freien Konstante $\mu_{0}$ abhängt. Man findet hier $\sqrt{\frac{\mu_{0}}{m}}\langle R\rangle=11,0 \AA$ im Grundzustand und $\sqrt{\frac{\mu_{0}}{m}}\langle R\rangle=101 \AA$ im angeregten Zustand.

\subsection{Die Streumatrix des Trimers}

Praktische Methoden für die Mehrteilchen- und Mehrkanal-Streutheorie wurden in den sechziger Jahren im Umfeld der theoretischen Kernphysik entwickelt. Als besonders nützlich für die Untersuchung von Dreiteilchen-Streuprozessen mit vorgegebenen 
einlaufenden und auslaufenden Zuständen erwies sich dabei der von Alt, Grassberger und Sandhas (AGS) entwickelte Zugang, der für diese Prozesse Übergangsoperatoren in Analogie zu den aus der Zweiteilchen-Streuung bekannten T-Matrizen bereitstellt [55]. Die Übergangsoperatoren erfüllen einen Satz von Integralgleichungen. Hegerfeldt und Köhler übertrugen und modifizierten den Zugang von AGS auf die Beugung schwach gebundener, diatomarer Moleküle (Dimere) an einem äußeren Potential [3, 4, 40]. In abstrakter Notation geschrieben sind diese modifizierten AGSGleichungen unabhängig von der Anzahl der Teilchen, solange sich nur die potentielle Energie im vollständigen Hamilton-Operator der Clusterbeugung zerlegen läßt in die zwei Anteile $V+W$, von denen $V$ die Summe aller Paarwechselwirkungen im Cluster beschreibt und $W$ die Summe der Wechselwirkungen aller Teilchen mit dem Beugungsobjekt. Aus diesem Grund gleichen die im folgenden für die Trimerbeugung abgeleiteten Formeln zunächst jenen der Dimerbeugung [3, 4, 40]. Allerdings ist zu berücksichtigen, daß die auftretenden Operatoren wie die Resolventen und die Übergangsoperatoren wegen des zusätzlichen Teilchens in einem größeren Hilbertraum wirken. Dieser Unterschied wird sich ab Abschnitt 3.3 bemerkbar machen.

\subsubsection{Modellierung eines Molekularstrahlexperiments}

In den Molekularstrahlexperimenten von Brühl, Kalinin, Kornilov und Toennies (vgl. Kap. 4) expandiert ein Atomgas unter definiertem Druck durch eine auf fester Temperatur gehaltene Düse. Innerhalb eines Raumgebiets von der Ausdehnung weniger Düsendurchmesser wechselwirken die Atome miteinander. Sie bilden zum Teil Cluster, deren relative Häufigkeiten vom Druck und der Temperatur abhängen [56, 57]. In etwas größerer Entfernung von der Düse ist das Gas aufgrund der Expansion bereits stark verdünnt. Andererseits befinden sich die Atome und Cluster dort noch sehr weit vom Beugungsobjekt, dem Transmissionsgitter, entfernt. Sie können zu diesem Zeitpunkt als freie Teilchen angesehen werden. Durch einen nach der Düse plazierten Skimmer wird ein feiner Strahl von Atomen und Clustern erzeugt. Eine detaillierte Beschreibung der experimentellen Apparatur wird in Ref. [58] gegeben. Eine Besonderheit des Heliums ist die außerordentlich große Streulänge der HeliumHelium-Streuung $a=104 \AA$ [5], die eine sehr scharfe Geschwindigkeitsverteilung von $\Delta v / v \approx 2 \%$ im Strahl erlaubt [18, 56, 59]. Die mittlere Geschwindigkeit der Atome und Cluster ist gleich.

Die Wechselwirkung des Trimers mit dem Transmissionsgitter wird durch ein Potential $W$ beschrieben, das sich zusammensetzt aus den einzelnen Wechselwirkungen $W_{i}$ der drei Atome mit dem Gitter:

$$
W\left(\mathbf{x}_{1}, \mathbf{x}_{2}, \mathbf{x}_{3}\right)=W_{1}\left(\mathbf{x}_{1}\right)+W_{2}\left(\mathbf{x}_{2}\right)+W_{3}\left(\mathbf{x}_{3}\right) .
$$

Prinzipiell wäre auch hier eine Mehrteilchenwechselwirkung zu berücksichtigen, die dieselbe Ursache wie die zu Beginn von Kapitel 3.1.1 erwähnte Dreikörperwechselwirkung im freien Trimer hat. Ohne Beweis wird im folgenden angenommen, daß ihr Einfluß ebenfalls vernachlässigt werden darf.

Zur Vereinfachung der Notation wird nun vereinbart, daß - wenn nicht explizit anders angegeben - die Realisierung $\boldsymbol{\rho} \equiv \boldsymbol{\rho}^{(1)}, \mathbf{r} \equiv \mathbf{r}^{(23)}$ der Jacobi-Koordinaten 
verwendet wird. Die Indizes der Koordinaten und auch die Kennzeichnung $(i, j k)$ an Funktionen werden im folgenden nur notiert, wenn sich andernfalls eine Mehrdeutigkeit ergäbe. Der vollständige Hamilton-Operator der Trimerbeugung lautet

$$
H=H_{0}+V+W
$$

mit der kinetischen Energie $H_{0}$ (3.13) und der Summe der Paarwechselwirkungen des Trimers $V$ (3.14).

Zum Zeitpunkt $t_{\mathrm{i}}$ habe ein Trimer gerade die Expansionsphase des Teilchenstrahls durchlaufen und werde durch einen Eigenzustand $\left|\psi\left(t_{\mathrm{i}}\right), \phi_{\gamma^{\prime}}\left(t_{\mathrm{i}}\right)\right\rangle$ des freien HamiltonOperators $H_{0}+V$ beschrieben. Die genaue Form des normierten Schwerpunktzustandes $\left|\psi\left(t_{\mathrm{i}}\right)\right\rangle$ wird im folgenden unerheblich sein und kann für alle Bindungszustände als gleich angenommen werden. Die Wiederholung des Experiments realisiert ein quantenmechanisches Ensemble, das durch den Dichte-Operator

$$
\rho\left(t_{\mathrm{i}}\right):=\sum_{\gamma^{\prime}} p_{\gamma^{\prime}}\left|\psi\left(t_{\mathrm{i}}\right), \phi_{\gamma^{\prime}}\left(t_{\mathrm{i}}\right)\right\rangle\left\langle\psi\left(t_{\mathrm{i}}\right), \phi_{\gamma^{\prime}}\left(t_{\mathrm{i}}\right)\right| \quad \text { mit } \quad \sum_{\gamma^{\prime}} p_{\gamma^{\prime}}=1
$$

beschrieben wird. Im Wechselwirkungsbild bezüglich des Gitterpotentials $W$ hat er die Form

$$
\mathrm{e}^{\mathrm{i}\left(H_{0}+V\right) t_{\mathrm{i}} / \hbar} \rho\left(t_{\mathrm{i}}\right) \mathrm{e}^{-\mathrm{i}\left(H_{0}+V\right) t_{\mathrm{i}} / \hbar}=: \sum_{\gamma^{\prime}} p_{\gamma^{\prime}}\left|\psi_{\text {in }}, \phi_{\gamma^{\prime}}\right\rangle\left\langle\psi_{\text {in }}, \phi_{\gamma^{\prime}}\right|=: \rho_{\text {in }} .
$$

Die Zeitentwicklung des Dichte-Operators wird durch den vollständigen HamiltonOperator (3.37) bestimmt. Aus (3.39) folgt

$$
\rho(t)=\mathrm{e}^{-\mathrm{i} H t / \hbar} \mathrm{e}^{\mathrm{i} H t_{\mathrm{i}} / \hbar} \mathrm{e}^{-\mathrm{i}\left(H_{0}+V\right) t_{\mathrm{i}} / \hbar} \rho_{\text {in }} \mathrm{e}^{\mathrm{i}\left(H_{0}+V\right) t_{\mathrm{i}} / \hbar} \mathrm{e}^{-\mathrm{i} H t_{\mathrm{i}} / \hbar} \mathrm{e}^{\mathrm{i} H t / \hbar} .
$$

Der genaue Zeitpunkt $t_{\mathrm{i}}$ ist allerdings nicht von Bedeutung. Mathematisch darf der Limes $t_{\mathrm{i}} \rightarrow-\infty$ ausgeführt werden. In der Literatur zur Streutheorie (z. B. Ref. [46]) werden in diesem Zusammenhang die Møller- oder Wellen-Operatoren

$$
\Omega_{V}^{ \pm}:=\lim _{t \rightarrow \mp \infty} \mathrm{e}^{\mathrm{i} H t / \hbar} \mathrm{e}^{-\mathrm{i}\left(H_{0}+V\right) t / \hbar} P_{V}
$$

eingeführt, wobei $P_{V}$ ein Projektor auf gebundene Trimer-Zustände ist. Mit ihrer Hilfe kann $\rho(t)$ geschrieben werden als

$$
\rho(t) \simeq \mathrm{e}^{-\mathrm{i} H t / \hbar} \Omega_{V}^{+} \rho_{\text {in }}\left(\Omega_{V}^{+}\right)^{\dagger} \mathrm{e}^{\mathrm{i} H t / \hbar} .
$$

Die Wahrscheinlichkeitsdichte dafür, daß das Trimer lange nach der Wechselwirkung mit dem Transmissionsgitter zum Zeitpunkt der Detektion $t_{\mathrm{f}}$ mit einem auslaufendem Impuls $\mathbf{P}$ im Bindungszustand $\phi_{\gamma}$ gemessen wird, ist durch den Ausdruck

$$
w\left(\mathbf{P}, \phi_{\gamma} ; \rho\right):=\left\langle\mathbf{P}, \phi_{\gamma}\left|\mathrm{e}^{-\mathrm{i} H t_{\mathrm{f}} / \hbar} \Omega_{V}^{+} \rho_{\text {in }}\left(\Omega_{V}^{+}\right)^{\dagger} \mathrm{e}^{\mathrm{i} H t_{\mathrm{f}} / \hbar}\right| \mathbf{P}, \phi_{\gamma}\right\rangle
$$

gegeben. Da $\left|\mathbf{P}, \phi_{\gamma}\right\rangle$ ein Eigenzustand von $H_{0}+V$ ist, kann das Matrixelement in (3.43) um zwei Exponentialfaktoren und Projektionsoperatoren $P_{V}$ ergänzt werden, 
so daß Produkte in der Form der Møller-Operatoren $\Omega_{V}^{-}$auftreten. Weil auch der genaue Detektionszeitpunkt nicht von Bedeutung ist, kann der Limes $t_{\mathrm{f}} \rightarrow \infty$ ausgeführt werden. Aus Gleichung (3.43) folgt dann

$$
w\left(\mathbf{P}, \phi_{\gamma} ; \rho\right)=\left\langle\mathbf{P}, \phi_{\gamma}\left|\left(\Omega_{V}^{-}\right)^{\dagger} \Omega_{V}^{+} \rho_{\text {in }}\left(\Omega_{V}^{+}\right)^{\dagger} \Omega_{V}^{-}\right| \mathbf{P}, \phi_{\gamma}\right\rangle .
$$

Der Operator $\left(\Omega_{V}^{-}\right)^{\dagger} \Omega_{V}^{+}$wird in der Streutheorie als Kanalstreumatrix zwischen gebundenen Zuständen des Potentials $V$ bezeichnet.

$$
S_{V V}:=\left(\Omega_{V}^{-}\right)^{\dagger} \Omega_{V}^{+}
$$

Er beschreibt Streuprozesse, deren einlaufende und auslaufende Zustände sich asymptotisch wie gebundene Trimere verhalten, die aber möglicherweise in verschiedenen Bindungszuständen vorliegen. Einsetzen der Zerlegung von $\rho_{\text {in }}$ nach Gleichung (3.39) führt schließlich auf das Ergebnis

$$
w\left(\mathbf{P}, \phi_{\gamma} ; \rho\right)=\sum_{\gamma^{\prime}} p_{\gamma^{\prime}}\left|\left\langle\mathbf{P}, \phi_{\gamma}\left|S_{V V}\right| \psi_{\text {in }}, \phi_{\gamma^{\prime}}\right\rangle\right|^{2} .
$$

Die Wahrscheinlichkeit, den Bindungszustand $\left|\phi_{\gamma}\right\rangle$ zu detektieren, hängt also sowohl von den relativen Besetzungszahlen $p_{\gamma^{\prime}}$ als auch von der Übergangswahrscheinlichkeit des Zustandes $\left|\phi_{\gamma^{\prime}}\right\rangle$ bei Wechselwirkung mit dem Potential $W$ in den Zustand $\left|\phi_{\gamma}\right\rangle$ ab. Der Hauptgegenstand dieses Kapitels ist die Berechnung des in Gleichung (3.46) auftretenden Streumatrixelementes.

\subsubsection{Das Streumatrixelement}

Die Gleichung (3.46) enthält den Term

$$
\left|\psi, \phi_{\gamma^{\prime}},+\right\rangle:=\Omega_{V}^{+}\left|\psi_{\mathrm{in}}, \phi_{\gamma^{\prime}}\right\rangle,
$$

der als zum Zustand $\left|\psi_{\text {in }}, \phi_{\gamma^{\prime}}\right\rangle$ gehörender Streuzustand bezeichnet wird. Durch Einsetzen der Definition (3.41) des Møller-Operators $\Omega_{V}^{+}$und Einschieben eines vollständigen Systems von Schwerpunktimpuls-Eigenzuständen $\int \mathrm{d}^{3} P^{\prime}\left|\mathbf{P}^{\prime}\right\rangle\left\langle\mathbf{P}^{\prime}\right|$ kann er ausgedrückt werden als

$$
\begin{aligned}
\left|\psi, \phi_{\gamma^{\prime}},+\right\rangle & =\lim _{t \rightarrow-\infty} \int \mathrm{d}^{3} P^{\prime} \psi\left(\mathbf{P}^{\prime}\right) \mathrm{e}^{\mathrm{i}\left(H-E^{\prime}\right) t / \hbar}\left|\mathbf{P}^{\prime}, \phi_{\gamma^{\prime}}\right\rangle \\
& =\lim _{\epsilon \downarrow 0} \int \mathrm{d}^{3} P^{\prime} \psi\left(\mathbf{P}^{\prime}\right) \frac{\mathrm{i} \epsilon}{E^{\prime}-H+\mathrm{i} \epsilon}\left|\mathbf{P}^{\prime}, \phi_{\gamma^{\prime}}\right\rangle,
\end{aligned}
$$

wobei in der zweiten Zeile der zeitliche Grenzwert durch den sogenannten Abel-Limes ersetzt wurde. Gleichung (3.48) gibt Anlaß zur Definition der Resolvente

$$
G(z):=[z-H]^{-1}
$$


für komplexe Zahlen $z$, die nicht im Spektrum von $H$ liegen. Darüberhinaus ist es nützlich, weitere Resolventen zu definieren,

$$
\begin{aligned}
G_{0}(z) & :=\left[z-H_{0}\right]^{-1} \\
G_{V}(z) & :=\left[z-H_{0}-V\right]^{-1} \\
G_{W}(z) & :=\left[z-H_{0}-W\right]^{-1},
\end{aligned}
$$

die gemeinsam mit $G(z)$ die Resolventengleichungen

$$
\begin{aligned}
& G(z)=G_{V}(z)+G_{V}(z) W G(z) \\
& G(z)=G_{W}(z)+G_{W}(z) V G(z)
\end{aligned}
$$

erfüllen. Weiterhin werden zwei Operatoren $T_{V}$ und $T_{W}$ definiert, die dieselbe Struktur wie $T$-Matrizen im Falle der Potentialstreuung an den Potentialen $V$ beziehungsweise $W$ aufweisen.

$$
\begin{aligned}
T_{V}(z) & :=V+V G_{V}(z) V \\
T_{W}(z) & :=W+W G_{W}(z) W
\end{aligned}
$$

Sie wirken allerdings, ebenso wie die Resolventen $G_{V}$ und $G_{W}$, im gesamten Hilbertraum $\mathcal{H}$, so daß sie neundimensionalen Matrizen entsprechen. Eine kurze Rechnung zeigt, daß $T_{V}$ und $T_{W}$ die Relationen

$$
V G_{V}=T_{V} G_{0} \quad \text { und } \quad W G_{W}=T_{W} G_{0}
$$

erfüllen.

Um auch für Trimere eine Zerlegung des Streumatrixelements in der Form (2.5) zu erhalten, werden „Übergangsoperatoren" $U_{V V}, U_{W V}$ durch die definierenden Gleichungen

$$
\begin{aligned}
& G(z)=: G_{V}(z)+G_{V}(z) U_{V V}(z) G_{V}(z) \\
& G(z)=: G_{W}(z) U_{W V}(z) G_{V}(z)
\end{aligned}
$$

eingeführt. Dieser Typ von Übergangsoperatoren geht ursprünglich auf Alt, Grassberger und Sandhas zurück [55]. Mit Hilfe der Resolventengleichungen (3.51a, 3.51b) und den Beziehungen (3.54) lassen sich daraus die modifizierten AGS-Gleichungen [3]

$$
\begin{array}{r}
U_{V V}=T_{W} G_{0} U_{W V} \\
U_{W V}=G_{0}^{-1}+T_{V} G_{0} U_{V V}
\end{array}
$$

für die Übergangsoperatoren ableiten, die nach einer Iteration eine entkoppelte Integralgleichung für $U_{V V}$ liefern:

$$
U_{V V}=T_{W}+T_{W} G_{0} T_{V} G_{0} U_{V V} .
$$

Da in dieser Integralgleichung die „starke“ Wechselwirkung mit dem Beugungsgitter $W$ im führenden Term einzeln auftritt und im zweiten Term nur in Verbindung 
mit dem „schwachen“ Trimer-Bindungspotential $V$, eignet sich die Gleichung für eine Störungsentwicklung [3, 40]. Hierin liegt der Vorteil der modifizierten AGSGleichungen.

Die mit der Darstellung des Trimer-Streuzustandes durch die Resolvente $G(z)$ begonnene Berechnung (3.48) des Streumatrixelements wird nun fortgesetzt. Unter Verwendung der Definition (3.41) des Møller-Operators $\Omega_{V}^{-}$lautet das Streumatrixelement

$$
\begin{aligned}
& \left\langle\mathbf{P}, \phi_{\gamma}\left|S_{V V}\right| \psi_{\text {in }}, \phi_{\gamma^{\prime}}\right\rangle= \\
& \quad \lim _{t \rightarrow+\infty} \lim _{\epsilon \downarrow 0} \int \mathrm{d}^{3} P^{\prime} \psi\left(\mathbf{P}^{\prime}\right) \mathrm{e}^{\mathrm{i}\left(E-E^{\prime}\right) t / \hbar} \mathrm{i} \epsilon\left\langle\mathbf{P}, \phi_{\gamma}\left|G\left(E^{\prime}+\mathrm{i} \epsilon\right)\right| \mathbf{P}^{\prime}, \phi_{\gamma^{\prime}}\right\rangle,
\end{aligned}
$$

wobei die Energien der uneigentlichen Zustände $\left|\mathbf{P}, \phi_{\gamma}\right\rangle$ durch Gleichung (3.16) gegeben sind. Die gesuchte Zerlegung des Streumatrixelements gelingt nun durch Einsetzen der Gleichung (3.55) für die Resolvente.

$$
\begin{aligned}
& \left\langle\mathbf{P}, \phi_{\gamma}\left|S_{V V}\right| \psi_{\text {in }}, \phi_{\gamma^{\prime}}\right\rangle=\lim _{t \rightarrow+\infty} \lim _{\epsilon \downarrow 0} \int \mathrm{d}^{3} P^{\prime} \psi\left(\mathbf{P}^{\prime}\right) \mathrm{e}^{\mathrm{i}\left(E-E^{\prime}\right) t / \hbar} \\
& \times\left[\delta^{(3)}\left(\mathbf{P}-\mathbf{P}^{\prime}\right) \delta_{\gamma \gamma^{\prime}}-\frac{1}{E-E^{\prime}-\mathrm{i} \epsilon}\left\langle\mathbf{P}, \phi_{\gamma}\left|U_{V V}\left(E^{\prime}+\mathrm{i} \epsilon\right)\right| \mathbf{P}^{\prime}, \phi_{\gamma^{\prime}}\right\rangle\right]
\end{aligned}
$$

Aufgrund der Distributionenidentität

$$
\lim _{t \rightarrow+\infty} \lim _{\epsilon \downarrow 0} \frac{\mathrm{e}^{\mathrm{i}\left(E-E^{\prime}\right) t / \hbar}}{E-E^{\prime}-\mathrm{i} \epsilon}=2 \pi \mathrm{i} \delta\left(E-E^{\prime}\right)
$$

vereinfacht sich Gleichung (3.58) zu

$$
\begin{aligned}
& \left\langle\mathbf{P}, \phi_{\gamma}\left|S_{V V}\right| \psi_{\mathrm{in}}, \phi_{\gamma^{\prime}}\right\rangle= \\
& \int \mathrm{d}^{3} P^{\prime} \psi\left(\mathbf{P}^{\prime}\right)\left[\delta^{(3)}\left(\mathbf{P}-\mathbf{P}^{\prime}\right) \delta_{\gamma \gamma^{\prime}}-2 \pi \mathrm{i} \delta\left(E-E^{\prime}\right) t\left(\mathbf{P}, \phi_{\gamma} ; \mathbf{P}^{\prime}, \phi_{\gamma^{\prime}}\right)\right],
\end{aligned}
$$

wobei die Trimer-Übergangsamplitude durch

$$
t\left(\mathbf{P}, \phi_{\gamma} ; \mathbf{P}^{\prime}, \phi_{\gamma^{\prime}}\right):=\left\langle\mathbf{P}, \phi_{\gamma}\left|U_{V V}\left(E^{\prime}+\mathrm{i} 0\right)\right| \mathbf{P}^{\prime}, \phi_{\gamma^{\prime}}\right\rangle
$$

definiert wurde. Die Notation i0 bedeutet die Ausführung des Limes $\epsilon \downarrow 0$ nach der Auswertung des Matrixelements von $U_{V V}$.

Nimmt man wiederum an, daß das Beugungsobjekt entlang der $x_{3}$-Achse translationsinvariant ist, so enthält die Integralgleichung (3.57) die Schwerpunktkomponente $\hat{R}_{3}$ nicht. Folglich ist $U_{V V}$ diagonal in $\left|P_{3}^{\prime}\right\rangle$, und die Übergangsamplitude (3.60) kann durch Ausfaktorisieren einer impulserhaltenden Deltafunktion zu

$$
t\left(\mathbf{P}, \phi_{\gamma} ; \mathbf{P}^{\prime}, \phi_{\gamma^{\prime}}\right)=: \delta\left(P_{3}-P_{3}^{\prime}\right) t^{\mathrm{red}}\left(\boldsymbol{P}, \phi_{\gamma} ; \boldsymbol{P}^{\prime}, \phi_{\gamma^{\prime}}\right)
$$

vereinfacht werden, wobei

$$
t^{\mathrm{red}}\left(\boldsymbol{P}, \phi_{\gamma} ; \boldsymbol{P}^{\prime}, \phi_{\gamma^{\prime}}\right)=\left\langle\boldsymbol{P}, \phi_{\gamma}\left|U_{V V}^{\mathrm{red}}\left(E^{\prime}-\frac{P_{3}^{\prime 2}}{2 M}+\mathrm{i} 0\right)\right| \boldsymbol{P}^{\prime}, \phi_{\gamma^{\prime}}\right\rangle
$$


die auf zwei Dimensionen reduzierte Übergangsamplitude ist. Der Übergangsoperator $U_{V V}^{\text {red }}$ ergibt sich aus dem Operator $U_{V V}$ durch Fortlassen aller Terme $\hat{P}_{3}^{2} / 2 M$. Analog zur Notation von Kapitel 2 repräsentieren die kursiv gesetzten Vektorsymbole $\boldsymbol{P}^{\prime}$ und $\boldsymbol{P}$ die zweidimensionalen Projektionen des einlaufenden und des auslaufenden Schwerpunktimpulses in die $\left(x_{1}, x_{2}\right)$-Ebene.

\subsection{Die Übergangsamplitude des Trimers}

In diesem Abschnitt wird die Übergangsamplitude eines schwach gebundenen Trimers, dessen drei Teilchenmassen von der gleichen Größenordnung sind, berechnet. Nach den Voraussetzungen für eine Störungsentwicklung des Übergangsoperators wird in Unterabschnitt 3.3.2 wird die allgemeine Form für ein in einer Richtung translationsinvariantes Beugungsobjekt abgeleitet, die anschließend im Unterabschnitt 3.3.3 auf die Situation der Beugung am Transmissionsgitter spezialisiert wird.

\subsubsection{Störungsentwicklung der Übergangsamplitude}

Zunächst wird vorausgesetzt, daß die kinetische Energie der Schwerpunktbewegung die Bindungsenergien und die Erwartungswerte der potentiellen Energie der zu untersuchenden Bindungszustände $\left|\phi_{\gamma^{\prime}}\right\rangle$ des Trimers wesentlich überschreitet.

$$
\frac{|\boldsymbol{P}|^{2}}{2 M}, \frac{\left|\boldsymbol{P}^{\prime}\right|^{2}}{2 M} \gg\left|E_{\gamma^{\prime}}\right|,\left|\left\langle\phi_{\gamma^{\prime}}|V| \phi_{\gamma^{\prime}}\right\rangle\right|
$$

Im speziellen Fall des Helium-Trimers ${ }^{4} \mathrm{He}_{3}$, das in den Experimenten von Brühl, Kalinin, Kornilov und Toennies bei Strahlgeschwindigkeiten von $250 \ldots 650 \mathrm{~m} / \mathrm{s}$ erzeugt werden konnte, beträgt die kinetische Energie beispielsweise $0,4 \ldots 3 \times 10^{-2} \mathrm{eV}$, wohingegen die theoretisch vorhergesagten Bindungsenergien bei $-1 \times 10^{-5} \mathrm{eV}$ im Grundzustand und $-2 \times 10^{-7} \mathrm{eV}$ im Anregungszustand liegen (vgl. Tab. 3.1). Die Voraussetzung (3.63) ist hier somit erfüllt. Dann ist es möglich, den reduzierten Übergangsoperator $U_{V V}^{\text {red }}(3.62)$ auf der Energieschale $z^{\text {red }}:=E^{\prime}-P_{3}^{\prime 2} / 2 M+\mathrm{i} 0$ durch den führenden Term

$$
\left\langle\boldsymbol{P}, \phi_{\gamma}\left|U_{V V}^{\mathrm{red}}\left(z^{\mathrm{red}}\right)\right| \boldsymbol{P}^{\prime}, \phi_{\gamma^{\prime}}\right\rangle \simeq\left\langle\boldsymbol{P}, \phi_{\gamma}\left|T_{W}^{\mathrm{red}}\left(z^{\mathrm{red}}\right)\right| \boldsymbol{P}^{\prime}, \phi_{\gamma^{\prime}}\right\rangle
$$

zu approximieren, wobei der Operator $T_{W}^{\text {red }}$ durch Fortlassen der Terme $\hat{P}_{3}^{2} / 2 M$ aus $T_{W}$ hervorgeht. Die Gültigkeit der Näherung (3.64) unter der Voraussetzung der geringen Bindungsenergie (3.63) wird in Ref. [40, Anh. B] für den Fall der Dimerbeugung ausführlich diskutiert und ist unmittelbar auf Trimere übertragbar. Sie folgt aus der Kleinheit des ersten bei Iteration von Gleichung (3.57) auftretenden Terms

$$
\left\langle\boldsymbol{P}, \phi_{\gamma}\left|T_{W}^{\mathrm{red}}\left(z^{\mathrm{red}}\right) G_{0}^{\mathrm{red}}\left(z^{\mathrm{red}}\right) T_{V}^{\mathrm{red}}\left(z^{\mathrm{red}}\right) G_{0}^{\mathrm{red}}\left(z^{\mathrm{red}}\right) T_{W}^{\mathrm{red}}\left(z^{\mathrm{red}}\right)\right| \boldsymbol{P}^{\prime}, \phi_{\gamma^{\prime}}\right\rangle,
$$

der eine Korrektur der Größenordnung $\left|\left\langle\phi_{\gamma}|V| \phi_{\gamma}\right\rangle\right| /\left(|\boldsymbol{P}|^{2} / 2 M\right)$ relativ zum führenden Term liefert. Das Trimer-Bindungspotential $V$ geht daraufhin nur noch indirekt über die Trimer-Bindungszustände in die Übergangsamplitude (3.64) ein. 
Eine weitere Aussage erhält man aus der Betrachtung des Relativanteils der Schrödinger-Gleichung des freien Trimers unter der Bedingung der großen kinetischen Energie und der zusätzlichen Bedingung, daß die drei Massen $m_{i}$ von derselben Größenordnung sind. Durch Multiplikation von

$$
\left[\frac{M}{2 m_{1}\left(m_{2}+m_{3}\right)} \hat{\mathbf{q}}^{2}+\frac{m_{2}+m_{3}}{2 m_{2} m_{3}} \hat{\mathbf{p}}^{2}+V\right]\left|\phi_{\gamma}\right\rangle=E_{\gamma}\left|\phi_{\gamma}\right\rangle
$$

mit $\left\langle\phi_{\gamma}\right|$ läßt sich zeigen, daß die Impulsraumdarstellung $\phi_{\gamma}(\mathbf{q}, \mathbf{p}) \equiv\left\langle\mathbf{q}, \mathbf{p} \mid \phi_{\gamma}\right\rangle$ der Bindungswellenfunktionen auf kleine Relativimpulse

$$
|\mathbf{q}| \ll|\boldsymbol{P}|,\left|\boldsymbol{P}^{\prime}\right| \quad \text { und } \quad|\mathbf{p}| \ll|\boldsymbol{P}|,\left|\boldsymbol{P}^{\prime}\right|
$$

konzentriert ist.

\subsubsection{Die zweidimensionale Übergangsamplitude}

In seiner Approximation durch den führenden Term ist der Übergangsoperator $U_{V V}^{\text {red }}$ auch diagonal in $\left|p_{3}^{\prime}\right\rangle$, da er das Trimer-Bindungspotential $V$ nicht mehr enthält. Die Projektionen der Relativimpulse in die $\left(x_{1}, x_{2}\right)$-Ebene werden nun wieder mit kursiven Vektorsymbolen bezeichnet. Durch Einschieben zweier vollständiger Systeme von Relativkoordinaten gelangt man von (3.64) zu dem Ausdruck

$$
\begin{aligned}
& t^{(2)}\left(\boldsymbol{P}, \phi_{\gamma} ; \boldsymbol{P}^{\prime}, \phi_{\gamma^{\prime}}\right):=\left\langle\boldsymbol{P}, \phi_{\gamma}\left|T_{W}^{\mathrm{red}}\left(z^{\mathrm{red}}\right)\right| \boldsymbol{P}^{\prime}, \phi_{\gamma^{\prime}}\right\rangle \\
&=\int \mathrm{d}^{3} q \mathrm{~d}^{3} p \int \mathrm{d} q_{1}^{\prime} \mathrm{d} q_{2}^{\prime} \mathrm{d} p_{1}^{\prime} \mathrm{d} p_{2}^{\prime} \phi_{\gamma}^{*}\left(\boldsymbol{q}, q_{3}, \boldsymbol{p}, p_{3}\right) \phi_{\gamma^{\prime}}\left(\boldsymbol{q}^{\prime}, q_{3}, \boldsymbol{p}^{\prime}, p_{3}\right) \\
& \times\left\langle\boldsymbol{P}, \boldsymbol{q}, \boldsymbol{p}\left|T_{W}^{(2)}\left(E^{\prime(2)}+\mathrm{i} 0\right)\right| \boldsymbol{P}^{\prime}, \boldsymbol{q}^{\prime}, \boldsymbol{p}^{\prime}\right\rangle
\end{aligned}
$$

mit der um die kinetische Energie aller Impulskomponenten bezüglich der dritten Koordinatenrichtung reduzierten Energie

$$
E^{\prime(2)}:=E^{\prime}-\frac{P_{3}^{\prime 2}}{2 M}-\frac{M}{2 m_{1}\left(m_{2}+m_{3}\right)} q_{3}^{\prime 2}-\frac{m_{2}+m_{3}}{2 m_{2} m_{3}} p_{3}^{\prime 2} .
$$

Der Operator $T_{W}^{(2)}$ in Gleichung (3.67) ist definiert durch

$$
T_{W}^{(2)}(z):=W+W G_{W}^{(2)}(z) W
$$

mit der Resolvente

$$
G_{W}^{(2)}(z):=\left[z-\frac{\hat{\boldsymbol{P}}^{2}}{2 M}-\frac{M}{2 m_{1}\left(m_{2}+m_{3}\right)} \hat{\boldsymbol{q}}^{2}-\frac{m_{2}+m_{3}}{2 m_{2} m_{3}} \hat{\boldsymbol{p}}^{2}-W\right]^{-1} .
$$

In Gleichung (3.67) wird $T_{W}^{(2)}$ bei der Energie $E^{\prime(2)}$ außerhalb der Energieschale ausgewertet. Allerdings sind die Bindungswellenfunktionen laut der Abschätzung (3.66) 
auf kleine Relativimpulse konzentriert. Für die Energie $E^{\prime(2)}$ gilt daher unter dem Integralzeichen von Gleichung (3.67)

$$
\begin{aligned}
E^{\prime(2)} & =\frac{\left|\boldsymbol{P}^{\prime}\right|^{2}}{2 M}-\frac{M}{2 m_{1}\left(m_{2}+m_{3}\right)} q_{3}^{\prime 2}-\frac{m_{2}+m_{3}}{2 m_{2} m_{3}} p_{3}^{\prime 2}+E_{\gamma^{\prime}} \\
& \simeq \frac{\left|\boldsymbol{P}^{\prime}\right|^{2}}{2 M} \\
& \simeq \frac{\left|\boldsymbol{P}^{\prime}\right|^{2}}{2 M}+\frac{M}{2 m_{1}\left(m_{2}+m_{3}\right)}\left|\boldsymbol{q}^{\prime}\right|^{2}+\frac{m_{2}+m_{3}}{2 m_{2} m_{3}}\left|\boldsymbol{p}^{\prime}\right|^{2} \\
& =\frac{\left|\boldsymbol{p}_{1}^{\prime}\right|^{2}}{2 m_{1}}+\frac{\left|\boldsymbol{p}_{2}^{\prime}\right|^{2}}{2 m_{2}}+\frac{\left|\boldsymbol{p}_{3}^{\prime}\right|^{2}}{2 m_{3}}=: E_{1}^{\prime(2)}+E_{2}^{\prime(2)}+E_{3}^{\prime(2)}
\end{aligned}
$$

Die Energiedifferenz $E_{1}^{\prime(2)}+E_{2}^{\prime(2)}+E_{3}^{\prime(2)}-E^{\prime(2)}$ ist klein gegen $E^{\prime(2)}$. Falls nun das Matrixelement

$$
\left\langle\boldsymbol{P}, \boldsymbol{q}, \boldsymbol{p}\left|T_{W}^{(2)}\left(E^{\prime(2)}+\mathrm{i} 0\right)\right| \boldsymbol{P}^{\prime}, \boldsymbol{q}^{\prime}, \boldsymbol{p}^{\prime}\right\rangle
$$

bei $E^{\prime(2)}$ gegenüber dieser Energiedifferenz langsam veränderlich ist, so sollte es näherungsweise möglich sein, $E^{\prime(2)}$ durch $E_{1}^{\prime(2)}+E_{2}^{\prime(2)}+E_{3}^{\prime(2)}$ zu ersetzen und das Matrixelement somit auf der Energieschale auszuwerten. Formal beschriebe es dann die unabhängige Streuung dreier Teilchen an ihren jeweiligen Potentialen $W_{i}$. Im Rahmen der Dimerbeugung wurde diese Approximation ebenfalls ausführlich untersucht [40, Anh. B] und die langsame Veränderlichkeit von $T_{W}^{(2)}$ nachgewiesen unter der Bedingung, daß die Einteilchen-Übergangsamplituden $\left\langle\boldsymbol{p}_{i}\left|W_{i}\right| \boldsymbol{p}_{i}^{\prime},+\right\rangle$ bei der Energie $E_{i}^{\prime(2)}$ keine Bindungsresonanzen besitzen. Dieses Resultat ist ebenfalls auf die Trimerbeugung übertragbar; daher wird hier auf eine erneute Diskussion verzichtet. Quantitativ ist der Approximationsfehler wiederum von der Größenordnung $\left|\left\langle\phi_{\gamma}|V| \phi_{\gamma}\right\rangle\right| /\left(|\boldsymbol{P}|^{2} / 2 M\right)$. Der Vorteil der Auswertung des Matrixelementes auf der Energieschale liegt in der Möglichkeit seiner Zerlegung in die prinzipiell bekannten Einteilchen-Übergangsamplituden. Wegen $T_{W}^{(2)}(z) G_{0}^{(2)}(z)=W G_{W}^{(2)}(z)$, wobei $G_{0}^{(2)}(z)$ analog zu (3.70) definiert ist, gilt also, ausgedrückt in den Einteilchen-Impulsen anstelle der Jacobi-Koordinaten,

$$
\begin{aligned}
\left\langle\boldsymbol{p}_{1}, \boldsymbol{p}_{2}, \boldsymbol{p}_{3}\left|T_{W}^{(2)}\left(E^{\prime(2)}+\mathrm{i} 0\right)\right| \boldsymbol{p}_{1}^{\prime}, \boldsymbol{p}_{2}^{\prime}, \boldsymbol{p}_{3}^{\prime}\right\rangle & \\
& \simeq\left\langle\boldsymbol{p}_{1}, \boldsymbol{p}_{2}, \boldsymbol{p}_{3}\left|T_{W}^{(2)}\left(E_{1}^{\prime(2)}+E_{2}^{\prime(2)}+E_{3}^{\prime(2)}+\mathrm{i} 0\right)\right| \boldsymbol{p}_{1}^{\prime}, \boldsymbol{p}_{2}^{\prime}, \boldsymbol{p}_{3}^{\prime}\right\rangle \\
& =\left\langle\boldsymbol{p}_{1}, \boldsymbol{p}_{2}, \boldsymbol{p}_{3}|W| \boldsymbol{p}_{1}^{\prime}, \boldsymbol{p}_{2}^{\prime}, \boldsymbol{p}_{3}^{\prime},+\right\rangle .
\end{aligned}
$$

Da der zum Potential $W$ gehörende Møller-Operator faktorisiert, kann der Streuzustand $\left|\boldsymbol{p}_{1}^{\prime}, \boldsymbol{p}_{2}^{\prime}, \boldsymbol{p}_{3}^{\prime},+\right\rangle$ als Produkt geschrieben werden:

$$
\left|\boldsymbol{p}_{1}^{\prime}, \boldsymbol{p}_{2}^{\prime}, \boldsymbol{p}_{3}^{\prime},+\right\rangle=\left|\boldsymbol{p}_{1}^{\prime},+\right\rangle_{1}\left|\boldsymbol{p}_{2}^{\prime},+\right\rangle_{2}\left|\boldsymbol{p}_{3}^{\prime},+\right\rangle_{3} .
$$

Die Ziffern an den Ket-Vektoren geben die Teilchenindizes an. Einsetzen in Gleichung (3.72) und Berücksichtigung der Form des Potentials (3.36) liefert

$$
\begin{aligned}
\left\langle\boldsymbol{p}_{1}, \boldsymbol{p}_{2}, \boldsymbol{p}_{3}|W| \boldsymbol{p}_{1}^{\prime}, \boldsymbol{p}_{2}^{\prime}, \boldsymbol{p}_{3}^{\prime},+\right\rangle= \\
{ }_{1}\left\langle\boldsymbol{p}_{1}\left|W_{1}\right| \boldsymbol{p}_{1}^{\prime},+\right\rangle_{12}\left\langle\boldsymbol{p}_{2} \mid \boldsymbol{p}_{2}^{\prime},+\right\rangle_{2}{ }_{3}\left\langle\boldsymbol{p}_{3} \mid \boldsymbol{p}_{3}^{\prime},+\right\rangle_{3} \\
+{ }_{1}\left\langle\boldsymbol{p}_{1} \mid \boldsymbol{p}_{1}^{\prime},+\right\rangle_{12}\left\langle\boldsymbol{p}_{2}\left|W_{2}\right| \boldsymbol{p}_{2}^{\prime},+\right\rangle_{2}{ }_{3}\left\langle\boldsymbol{p}_{3} \mid \boldsymbol{p}_{3}^{\prime},+\right\rangle_{3} \\
+{ }_{1}\left\langle\boldsymbol{p}_{1} \mid \boldsymbol{p}_{1}^{\prime},+\right\rangle_{12}\left\langle\boldsymbol{p}_{2} \mid \boldsymbol{p}_{2}^{\prime},+\right\rangle_{2}\left\langle\boldsymbol{p}_{3}\left|W_{3}\right| \boldsymbol{p}_{3}^{\prime},+\right\rangle_{3} .
\end{aligned}
$$


Mit Hilfe der Impulsraumdarstellung der Lippmann-Schwinger-Gleichung für den uneigentlichen Einteilchen-Streuzustand des $i$. Teilchens (vgl. Kap. 2.1),

$$
{ }_{i}\left\langle\boldsymbol{p}_{i} \mid \boldsymbol{p}_{i}^{\prime},+\right\rangle_{i}=\delta^{(2)}\left(\boldsymbol{p}_{i}-\boldsymbol{p}_{i}^{\prime}\right)+\frac{{ }_{i}\left\langle\boldsymbol{p}_{i}\left|W_{i}\right| \boldsymbol{p}_{i}^{\prime}+\right\rangle_{i}}{E_{i}^{\prime(2)}-E_{i}^{(2)}+\mathrm{i} 0},
$$

und der Definition der zweidimensionalen Einteilchen-Übergangsamplitude durch

$$
t_{i}^{\text {at(2) }}\left(\boldsymbol{p}_{i} ; \boldsymbol{p}_{i}^{\prime}\right):={ }_{i}\left\langle\boldsymbol{p}_{i}\left|W_{i}\right| \boldsymbol{p}_{i}^{\prime},+\right\rangle_{i}
$$

folgt durch Ausmultiplizieren

$$
\begin{aligned}
\left\langle\boldsymbol{p}_{1}, \boldsymbol{p}_{2}, \boldsymbol{p}_{3}|W| \boldsymbol{p}_{1}^{\prime}, \boldsymbol{p}_{2}^{\prime}, \boldsymbol{p}_{3}^{\prime},+\right\rangle= & t_{1}^{\mathrm{at}(2)}\left(\boldsymbol{p}_{1} ; \boldsymbol{p}_{1}^{\prime}\right) \delta^{(2)}\left(\boldsymbol{p}_{2}-\boldsymbol{p}_{2}^{\prime}\right) \delta^{(2)}\left(\boldsymbol{p}_{3}-\boldsymbol{p}_{3}^{\prime}\right) \\
+ & t_{1}^{\mathrm{at}(2)}\left(\boldsymbol{p}_{1} ; \boldsymbol{p}_{1}^{\prime}\right) \delta^{(2)}\left(\boldsymbol{p}_{2}-\boldsymbol{p}_{2}^{\prime}\right) t_{3}^{\mathrm{at}(2)}\left(\boldsymbol{p}_{3} ; \boldsymbol{p}_{3}^{\prime}\right) \\
& \times\left[\frac{1}{E_{3}^{\prime(2)}-E_{3}^{(2)}+\mathrm{i} 0}+\frac{1}{E_{1}^{\prime(2)}-E_{1}^{(2)}+\mathrm{i} 0}\right] \\
+ & t_{1}^{\mathrm{att}(2)}\left(\boldsymbol{p}_{1} ; \boldsymbol{p}_{1}^{\prime}\right) t_{2}^{\mathrm{at}(2)}\left(\boldsymbol{p}_{2} ; \boldsymbol{p}_{2}^{\prime}\right) t_{3}^{\mathrm{at}(2)}\left(\boldsymbol{p}_{3} ; \boldsymbol{p}_{3}^{\prime}\right) \\
& \times \frac{1}{E_{2}^{\prime(2)}-E_{2}^{(2)}+\mathrm{i} 0} \frac{1}{E_{3}^{\prime(2)}-E_{3}^{(2)}+\mathrm{i} 0} \\
+ & \left(\begin{array}{c}
123 \\
231
\end{array}\right)+\left(\begin{array}{c}
123 \\
312
\end{array}\right) .
\end{aligned}
$$

Die abkürzende Schreibweise mit Hilfe der Symbole $\left(\begin{array}{l}123 \\ 231\end{array}\right)$ und $\left(\begin{array}{l}123 \\ 312\end{array}\right)$ soll bedeuten, daß allen drei Summanden auf der rechten Seite von Gleichung (3.74) zwei weitere mit jeweils aufsteigend permutierten Teilchenindizes hinzuzufügen sind. Mit Hilfe der sogenannten Hauptwertrelation [60]

$$
\frac{1}{x+\mathrm{i} 0}=-\mathrm{i} \pi \delta(x)+\mathcal{P} \frac{1}{x}
$$

kann im zweiten Summanden von Gleichung (3.74) die Ersetzung

$$
\begin{aligned}
& \delta^{(2)}\left(\boldsymbol{p}_{2}-\boldsymbol{p}_{2}^{\prime}\right)\left[\frac{1}{E_{3}^{\prime(2)}-E_{3}^{(2)}+\mathrm{i} 0}+\frac{1}{E_{1}^{\prime(2)}-E_{1}^{(2)}+\mathrm{i} 0}\right] \\
& =\delta^{(2)}\left(\boldsymbol{p}_{2}-\boldsymbol{p}_{2}^{\prime}\right)\left[-\mathrm{i} \pi \delta\left(E_{3}^{\prime(2)}-E_{3}^{(2)}\right)-\mathrm{i} \pi \delta\left(E_{1}^{(2)}-E_{1}^{(2)}\right)\right. \\
& \left.+\mathcal{P} \frac{1}{E_{3}^{\prime(2)}-E_{3}^{(2)}}+\mathcal{P} \frac{1}{E_{1}^{\prime(2)}-E_{1}^{(2)}}\right] \\
& \simeq \delta^{(2)}\left(\boldsymbol{p}_{2}-\boldsymbol{p}_{2}^{\prime}\right)\left[-2 \pi \mathrm{i} \delta\left(E_{1}^{\prime(2)}-E_{1}^{(2)}\right)\right]
\end{aligned}
$$

vorgenommen werden, wobei sich in der letzten Zeile wegen

$$
\epsilon:=E_{1}^{(2)}-E_{1}^{\prime(2)}+E_{2}^{(2)}-E_{2}^{\prime(2)}+E_{3}^{(2)}-E_{3}^{\prime(2)} \ll E^{(2)}, E^{\prime(2)}
$$

sowie der langsamen Veränderlichkeit der Einteilchen-Übergangsamplituden näherungsweise die Hauptwert-Terme gegenseitig herausheben. Der dabei verursachte 
Fehler ist proportional zu $\epsilon / E^{\prime(2)}$ und somit von derselben Größenordnung wie bei den bisherigen Approximationen (3.64) und (3.72). Die in Gleichung (3.74) auftretenden Produkte von Energie-Nennern können ebenfalls approximiert werden: die Distributionenidentität

$$
\begin{aligned}
\frac{1}{x+\mathrm{i} 0} \frac{1}{y+\mathrm{i} 0}+\frac{1}{y+\mathrm{i} 0} \frac{1}{z+\mathrm{i} 0} & +\frac{1}{z+\mathrm{i} 0} \frac{1}{x+\mathrm{i} 0} \\
& =-\frac{(2 \pi)^{2}}{3}[\delta(x) \delta(y)+\delta(y) \delta(z)+\delta(z) \delta(x)],
\end{aligned}
$$

kann für $x+y+z=0$ mittels Fourier-Transformation nachgewiesen werden. Für den Fall $x+y+z=\epsilon$, wobei $x=E_{1}^{\prime(2)}-E_{1}^{(2)}$ zu identifizieren ist etc., treten zusätzliche Terme auf, die ebenfalls von der Größenordnung $\epsilon / E^{\prime(2)}$ sind. Aus Gleichung (3.72) folgt somit

$$
\begin{aligned}
\left\langle\boldsymbol{p}_{1},\right. & \left.\boldsymbol{p}_{2}, \boldsymbol{p}_{3}\left|T_{W}^{(2)}\left(E^{\prime(2)}+\mathrm{i} 0\right)\right| \boldsymbol{p}_{1}^{\prime}, \boldsymbol{p}_{2}^{\prime}, \boldsymbol{p}_{3}^{\prime}\right\rangle \simeq \\
& t_{1}^{\mathrm{at}(2)}\left(\boldsymbol{p}_{1} ; \boldsymbol{p}_{1}^{\prime}\right) \delta^{(2)}\left(\boldsymbol{p}_{2}-\boldsymbol{p}_{2}^{\prime}\right) \delta^{(2)}\left(\boldsymbol{p}_{3}-\boldsymbol{p}_{3}^{\prime}\right) \\
- & 2 \pi \mathrm{i} \delta\left(E_{1}^{\prime(2)}-E_{1}^{(2)}\right) t_{1}^{\mathrm{at}(2)}\left(\boldsymbol{p}_{1} ; \boldsymbol{p}_{1}^{\prime}\right) \delta^{(2)}\left(\boldsymbol{p}_{2}-\boldsymbol{p}_{2}^{\prime}\right) t_{3}^{\mathrm{at}(2)}\left(\boldsymbol{p}_{3} ; \boldsymbol{p}_{3}^{\prime}\right) \\
- & \frac{(2 \pi)^{2}}{3} \delta\left(E_{2}^{\prime(2)}-E_{2}^{(2)}\right) \delta\left(E_{3}^{\prime(2)}-E_{3}^{(2)}\right) t_{1}^{\mathrm{at}(2)}\left(\boldsymbol{p}_{1} ; \boldsymbol{p}_{1}^{\prime}\right) t_{2}^{\mathrm{at}(2)}\left(\boldsymbol{p}_{2} ; \boldsymbol{p}_{2}^{\prime}\right) t_{3}^{\mathrm{at}(2)}\left(\boldsymbol{p}_{3} ; \boldsymbol{p}_{3}^{\prime}\right) \\
+ & \left(\begin{array}{c}
123 \\
231
\end{array}\right)+\left(\begin{array}{c}
123 \\
312
\end{array}\right) .
\end{aligned}
$$

Mit Hilfe der Transformation (3.10) werden die Einteilchen-Impulse nun wieder durch die Relativ- und Schwerpunktimpulse ausgedrückt und das Matrixelement (3.76) in die Trimer-Übergangsamplitude (3.67) eingesetzt. Es wird sich im folgenden als übersichtlich erweisen, die Argumente der Einteilchen-Übergangsamplituden durch den auslaufenden Einteilchen-Impuls $\boldsymbol{p}_{i}$ und den Impulsübertrag $\Delta \boldsymbol{p}_{i}=\boldsymbol{p}_{i}-\boldsymbol{p}_{i}^{\prime}$ auszudrücken.

$$
\widetilde{t}_{i}^{\mathrm{at}(2)}\left(\boldsymbol{p}_{i} ; \Delta \boldsymbol{p}_{i}\right):=t_{i}^{\mathrm{at}(2)}\left(\boldsymbol{p}_{i} ; \boldsymbol{p}_{i}^{\prime}\right)
$$

Damit folgt für die Übergangsamplitude (3.67) des Trimers

$$
\begin{gathered}
t^{(2)}\left(\boldsymbol{P}, \phi_{\gamma} ; \boldsymbol{P}^{\prime}, \phi_{\gamma^{\prime}}\right) \simeq \int \mathrm{d}^{3} q \mathrm{~d}^{3} p \int \mathrm{d} q_{1}^{\prime} \mathrm{d} q_{2}^{\prime} \mathrm{d} p_{1}^{\prime} \mathrm{d} p_{2}^{\prime} \phi_{\gamma}^{*}\left(\boldsymbol{q}, q_{3}, \boldsymbol{p}, p_{3}\right) \phi_{\gamma^{\prime}}\left(\boldsymbol{q}^{\prime}, q_{3}, \boldsymbol{p}^{\prime}, p_{3}\right) \\
\times\left\{\widetilde{t}_{1}^{\mathrm{at}(2)}\left(\frac{m_{1}}{M} \boldsymbol{P}+\boldsymbol{q} ; \frac{m_{1}}{M} \Delta \boldsymbol{P}+\Delta \boldsymbol{q}\right) \delta^{(2)}\left(\frac{m_{2}}{M} \Delta \boldsymbol{P}-\frac{m_{2}}{m_{2}+m_{3}} \Delta \boldsymbol{q}+\Delta \boldsymbol{p}\right)\right. \\
\times \delta^{(2)}\left(\frac{m_{3}}{M} \Delta \boldsymbol{P}-\frac{m_{3}}{m_{2}+m_{3}} \Delta \boldsymbol{q}-\Delta \boldsymbol{p}\right) \\
-2 \pi \mathrm{i} \widetilde{t}_{1}^{\mathrm{at}(2)}\left(\frac{m_{1}}{M} \boldsymbol{P}+\boldsymbol{q} ; \frac{m_{1}}{M} \Delta \boldsymbol{P}+\Delta \boldsymbol{q}\right) \delta^{(2)}\left(\frac{m_{2}}{M} \Delta \boldsymbol{P}-\frac{m_{2}}{m_{2}+m_{3}} \Delta \boldsymbol{q}+\Delta \boldsymbol{p}\right) \\
\times 2 m_{1} \delta\left(\left(\frac{m_{1}}{M} \boldsymbol{P}+\boldsymbol{q}\right)^{2}-\left(\frac{m_{1}}{M} \boldsymbol{P}^{\prime}+\boldsymbol{q}^{\prime}\right)^{2}\right) \\
\times \widetilde{t}_{3}^{\mathrm{at}(2)}\left(\frac{m_{3}}{M} \boldsymbol{P}-\frac{m_{3}}{m_{2}+m_{3}} \boldsymbol{q}-\boldsymbol{p} ; \frac{m_{3}}{M} \Delta \boldsymbol{P}-\frac{m_{3}}{m_{2}+m_{3}} \Delta \boldsymbol{q}-\Delta \boldsymbol{p}\right)
\end{gathered}
$$




$$
\begin{aligned}
-\frac{(2 \pi)^{2}}{3} & 4 m_{2} m_{3} \\
& \times \delta\left(\left(\frac{m_{2}}{M} \boldsymbol{P}-\frac{m_{2}}{m_{2}+m_{3}} \boldsymbol{q}+\boldsymbol{p}\right)^{2}-\left(\frac{m_{2}}{M} \boldsymbol{P}^{\prime}-\frac{m_{2}}{m_{2}+m_{3}} \boldsymbol{q}^{\prime}+\boldsymbol{p}^{\prime}\right)^{2}\right) \\
& \times \delta\left(\left(\frac{m_{3}}{M} \boldsymbol{P}-\frac{m_{3}}{m_{2}+m_{3}} \boldsymbol{q}-\boldsymbol{p}\right)^{2}-\left(\frac{m_{3}}{M} \boldsymbol{P}^{\prime}-\frac{m_{3}}{m_{2}+m_{3}} \boldsymbol{q}^{\prime}-\boldsymbol{p}^{\prime}\right)^{2}\right) \\
& \times \widetilde{t}_{1}^{\mathrm{at}(2)}\left(\frac{m_{1}}{M} \boldsymbol{P}+\boldsymbol{q} ; \frac{m_{1}}{M} \Delta \boldsymbol{P}+\Delta \boldsymbol{q}\right) \\
& \times \widetilde{t}_{2}^{\mathrm{at}(2)}\left(\frac{m_{2}}{M} \boldsymbol{P}-\frac{m_{2}}{m_{2}+m_{3}} \boldsymbol{q}+\boldsymbol{p} ; \frac{m_{2}}{M} \Delta \boldsymbol{P}-\frac{m_{2}}{m_{2}+m_{3}} \Delta \boldsymbol{q}+\Delta \boldsymbol{p}\right) \\
& \left.\times \widetilde{t}_{3}^{\mathrm{at}(2)}\left(\frac{m_{3}}{M} \boldsymbol{P}-\frac{m_{3}}{m_{2}+m_{3}} \boldsymbol{q}-\boldsymbol{p} ; \frac{m_{3}}{M} \Delta \boldsymbol{P}-\frac{m_{3}}{m_{2}+m_{3}} \Delta \boldsymbol{q}-\Delta \boldsymbol{p}\right)\right\} \\
+\left(\begin{array}{l}
123 \\
231
\end{array}\right) & +\left(\begin{array}{l}
123 \\
312
\end{array}\right) .
\end{aligned}
$$

Hier steht $\Delta \boldsymbol{P}:=\boldsymbol{P}-\boldsymbol{P}^{\prime}$ für den Impulsübertrag des Schwerpunkts in der Ebene; entsprechend sind die Impulsüberträge $\Delta \boldsymbol{q}:=\boldsymbol{q}-\boldsymbol{q}^{\prime}$ und $\Delta \boldsymbol{p}:=\boldsymbol{p}-\boldsymbol{p}^{\prime}$ definiert. Im ersten Summanden von (3.78) lassen sich aufgrund der Impuls-Deltafunktionen die Integrale über sowohl $\boldsymbol{q}^{\prime}$ als auch $\boldsymbol{p}^{\prime}$ ausführen. Im zweiten Summanden kann die Integration über $\boldsymbol{q}^{\prime}$ ausgeführt werden; die Energie-Deltafunktion wird dadurch zu

$$
\delta\left(\left(\frac{m_{1}}{M} \boldsymbol{P}+\boldsymbol{q}\right)^{2}-\left(\boldsymbol{P}^{\prime}-\frac{m_{2}+m_{3}}{M} \boldsymbol{P}+\boldsymbol{q}-\frac{m_{2}+m_{3}}{m_{2}} \Delta \boldsymbol{p}\right)^{2}\right) .
$$

Da die Einteilchen-Übergangsamplituden (2.42/2.79) um die Vorwärtsrichtung konzentriert sind, empfiehlt es sich hier analog zum Vorgehen in der Atombeugung, die Impulse durch ihre Komponenten parallel und senkrecht zum einfallenden Schwerpunktimpuls $\boldsymbol{P}^{\prime}$ auszudrücken. Die Nullstellen des Arguments der Deltafunktion (3.79) liegen bei

$$
\frac{m_{2}+m_{3}}{m_{2}} \Delta p_{\|}=P_{\|}^{\prime}-\frac{m_{2}+m_{3}}{M} P_{\|}+q_{\|} \pm \alpha_{1}\left|\frac{m_{1}}{M} P_{\|}+q_{\|}\right|,
$$

wobei zur Abkürzung der Schreibweise

$$
\alpha_{1}:=\sqrt{1+\frac{\left(\frac{m_{1}}{M} P_{\perp}+q_{\perp}\right)^{2}-\left(-\frac{m_{2}+m_{3}}{M} P_{\perp}+q_{\perp}-\frac{m_{2}+m_{3}}{m_{2}} \Delta p_{\perp}\right)^{2}}{\left(\frac{m_{1}}{M} P_{\|}+q_{\|}\right)^{2}}}
$$

definiert wurde. Aufgrund der Konzentration der Bindungswellenfunktionen auf kleine Relativimpulse (3.66) darf von den beiden Nullstellen (3.80) die zu „,+" gehörende bei $\frac{m_{2}+m_{3}}{m_{2}} \Delta p_{\|} \approx P_{\|}^{\prime}+\frac{\alpha_{1} m_{1}-m_{2}-m_{3}}{M} P_{\|}$entsprechend $\Delta p_{\|} \approx \frac{1}{3} P_{\|}^{\prime}$ vernachlässigt werden; außerdem können die Betragstriche in Gleichung (3.80) durch gewöhnliche Klammern ersetzt werden. Die Deltafunktion (3.79) wird also ersetzt durch

$$
\frac{\delta\left(\Delta P_{\|}+\frac{m_{2}+m_{3}}{m_{2}} \Delta p_{\|}+\left(\alpha_{1}-1\right)\left(\frac{m_{1}}{M} P_{\|}+q_{\|}\right)\right)}{2 \alpha_{1} \frac{m_{2}+m_{3}}{m_{2}}\left(\frac{m_{1}}{M} P_{\|}+q_{\|}\right)} .
$$


Da $\alpha_{1}-1=\mathcal{O}\left(P_{\perp} / P_{\|}\right)^{2}$, wird im folgenden untersucht, unter welchen Umständen näherungsweise $\alpha_{1}=1$ gesetzt werden darf. Zu diesem Zweck wird nur der zweite Summand aus Gleichung (3.78) betrachtet. Nach dem Ausintegrieren von $\boldsymbol{q}^{\prime}$ und $p_{\|}^{\prime}$ lautet er

$$
\begin{aligned}
& -4 \pi \mathrm{i} \frac{m_{1}\left(m_{2}+m_{3}\right)}{m_{2}} \int \mathrm{d}^{3} q \mathrm{~d}^{3} p \phi_{\gamma}^{*}\left(\boldsymbol{q}, q_{3}, \boldsymbol{p}, p_{3}\right) \frac{1}{2 \alpha_{1}\left(\frac{m_{1}}{M} P_{\|}+q_{\|}\right)} \int \mathrm{d} p_{\perp}^{\prime} \\
& \times \phi_{\gamma^{\prime}}\left(\frac{m_{1}}{M} \Delta P_{\|}+q_{\|}+\left(\alpha_{1}-1\right)\left(\frac{m_{1}}{M} P_{\|}+q_{\|}\right), q_{\perp}-\frac{m_{2}+m_{3}}{m_{2}}\left(\frac{m_{2}}{M} \Delta P_{\perp}+\Delta p_{\perp}\right),\right. \\
& \left.\quad q_{3}, \frac{m_{2}}{m_{2}+m_{3}} \Delta P_{\|}+p_{\|}+\left(\alpha_{1}-1\right) \frac{m_{2}}{m_{2}+m_{3}}\left(\frac{m_{1}}{M} P_{\|}+q_{\|}\right), p_{\perp}^{\prime}, p_{3}\right) \\
& \times \widetilde{t}_{1}^{\mathrm{at}(2)}\left(\frac{m_{1}}{M} \boldsymbol{P}+\boldsymbol{q} ;\left(1-\alpha_{1}\right)\left(\frac{m_{1}}{M} P_{\|}+q_{\|}\right), \Delta P_{\perp}+\frac{m_{2}+m_{3}}{m_{2}} \Delta p_{\perp}\right) \\
& \times \widetilde{t}_{3}^{\mathrm{at}(2)}\left(\frac{m_{3}}{M} \boldsymbol{P}-\frac{m_{3}}{m_{2}+m_{3}} \boldsymbol{q}-\boldsymbol{p} ; \Delta P_{\|}-\left(1-\alpha_{1}\right)\left(\frac{m_{1}}{M} P_{\|}+q_{\|}\right),-\frac{m_{2}+m_{3}}{m_{2}} \Delta p_{\perp}\right) .
\end{aligned}
$$

Entwicklung der Wurzel (3.81) liefert nun

$$
\alpha_{1}-1=-\frac{\left(m_{2}+m_{3}\right)^{2}-m_{1}^{2}}{2 m_{1}^{2}} \frac{P_{\perp}^{2}}{P_{\|}^{2}}+\mathcal{O}\left(\frac{q_{\perp}^{2}}{P_{\|}^{2}}, \frac{\left(\Delta p_{\perp}\right)^{2}}{P_{\|}^{2}}\right)+\mathcal{O}\left(\frac{P_{\perp}^{4}}{P_{\|}^{4}}\right) .
$$

Da die Einteilchen-Übergangsamplituden auf einen Impulsbereich der Breite $\Delta P_{\perp} \approx$ $4 \pi \hbar / s \cos \theta^{\prime}$ um die Vorwärtsrichtung konzentriert sind, wobei $s$ eine typische Länge des Beugungsobjektes ist, gilt im Fall gleicher Massen

$$
\left|\alpha_{1}-1\right| \approx 6\left(\frac{\lambda_{\mathrm{dB}}}{s \cos \theta^{\prime}}\right)^{2}
$$

Nach Voraussetzung der Beugungsbedingung (2.10) ist dieses Verhältnis klein; für die in Kapitel 4 auszuwertenden Experimente findet man beispielsweise $\left|\alpha_{1}-1\right| \approx$ $2 \times 10^{-5}$. Die Einteilchen-Übergangsamplituden in (3.82) werden also einerseits bei geringfügig verschobenem einlaufenden Impuls und deswegen andererseits bei geänderter Parallelkomponente des Einteilchen-Impulsübertrags $\Delta p_{i \|}$ ausgewertet. Wegen der langsamen Veränderlichkeit der Einteilchen-Übergangsamplituden im einlaufenden Impuls bei konstantem Impulsübertrag ist erstere Verschiebung vernachlässigbar. In der Parallelkomponente des dritten Teilchens ist hingegen die Verschiebung des Impulsübertrags $\left(1-\alpha_{1}\right) \frac{m_{1}}{M} P_{\|}=P_{\|} \mathcal{O}\left(P_{\perp} / P_{\|}\right)^{2}$ von derselben Größenordnung wie der Impulsübertrag $\Delta P_{\|}=P_{\|} \mathcal{O}\left(P_{\perp} / P_{\|}\right)^{2}$. Allerdings geht die Parallelkomponente des einlaufenden Impulses am Ende nur in der Form einer Summe mit der um das Verhältnis $\left(P_{\perp} / P_{\|}\right)^{-1}$ größeren Orthogonalkomponente in die EinteilchenÜbergangsamplitude ein, wie an deren Gleichung (2.42) und den Definitionen für die Spaltamplitude (2.40) und die Transmissionsfunktion des Spalts (2.32) zu erkennen ist. Aus diesem Grund ist eine Verschiebung der Parallelkomponente in ihrer eigenen Größenordnung vernachlässigbar.

Ebenfalls von der Größenordnung $P_{\|} \mathcal{O}\left(P_{\perp} / P_{\|}\right)^{2}$ sind die Impulsverschiebungen, mit denen die Bindungswellenfunktion $\phi_{\gamma^{\prime}}$ ausgewertet wird. Da das Interesse dieser 
Arbeit schwach gebundenen Trimeren mit großer räumlicher Ausdehnung gilt, insbesondere dem Helium-Trimer mit einem mittleren Paarabstand im Grundzustand von $\langle|\mathbf{r}|\rangle \approx 10 \AA$ (vgl. Tab. 3.1), ist aufgrund der Unschärferelation anzunehmen, daß $\phi_{\gamma^{\prime}}(\mathbf{q}, \mathbf{p})$ im Impulsraum Breiten der Größenordnung $\hbar /\langle|\boldsymbol{\rho}|\rangle$ beziehungsweise $\hbar /\langle|\mathbf{r}|\rangle$ aufweist. Die diskutierte Impulsverschiebung findet aber auf wesentlich kleineren Skalen statt. Es liegt daher nahe anzunehmen, daß die Bindungswellenfunktion gegenüber der Verschiebung langsam veränderlich ist. Am Beispiel der in Anhang B numerisch berechneten Bindungswellenfunktion des Helium-Trimers kann die Gültigkeit dieser Annahme direkt verifiziert werden. Konsequenterweise wird in den Argumenten der Bindungswellenfunktion die Parallelkomponente $\Delta P_{\|}$insgesamt vernachlässigt. Zusammenfassend ist es also möglich, den Ausdruck (3.82) näherungsweise bei $\alpha_{1}=1$ auszuwerten.

Entsprechend dem Vorgehen unterhalb von Gleichung (3.79) werden die im dritten Summanden der Trimer-Übergangsamplitude (3.78) auftretenden Energie-Deltafunktionen behandelt. So erhält man für die von $\delta\left(E_{2}^{\prime(2)}-E_{2}^{(2)}\right)$ herrührende Deltafunktion

$$
\begin{aligned}
\delta\left(\left(\frac{m_{2}}{M} \boldsymbol{P}-\frac{m_{2}}{m_{2}+m_{3}} \boldsymbol{q}+\boldsymbol{p}\right)^{2}-\left(\frac{m_{2}}{M} \boldsymbol{P}^{\prime}-\frac{m_{2}}{m_{2}+m_{3}} \boldsymbol{q}^{\prime}+\boldsymbol{p}^{\prime}\right)^{2}\right) \\
\simeq \frac{\delta\left(\frac{m_{2}}{M} \Delta P_{\|}-\frac{m_{2}}{m_{2}+m_{3}} \Delta q_{\|}+\Delta p_{\|}+\left(\alpha_{2}-1\right)\left(\frac{m_{2}}{M} P_{\|}-\frac{m_{2}}{m_{2}+m_{3}} q_{\|}+p_{\|}\right)\right)}{2 \alpha_{2}\left(\frac{m_{2}}{M} P_{\|}-\frac{m_{2}}{m_{2}+m_{3}} q_{\|}+p_{\|}\right)}
\end{aligned}
$$

mit

$$
\alpha_{2}:=\sqrt{1+\frac{\left(\frac{m_{2}}{M} P_{\perp}-\frac{m_{2}}{m_{2}+m_{3}} q_{\perp}+p_{\perp}\right)^{2}-\left(-\frac{m_{2}}{m_{2}+m_{3}} q_{\perp}^{\prime}+p_{\perp}^{\prime}\right)^{2}}{\left(\frac{m_{2}}{M} P_{\|}-\frac{m_{2}}{m_{2}+m_{3}} q_{\|}+p_{\|}\right)^{2}}} ;
$$

für die von $\delta\left(E_{3}^{\prime(2)}-E_{3}^{(2)}\right)$ herrührende Deltafunktion findet man

$$
\begin{aligned}
\delta\left(\left(\frac{m_{3}}{M} \boldsymbol{P}-\frac{m_{3}}{m_{2}+m_{3}} \boldsymbol{q}-\boldsymbol{p}\right)^{2}-\left(\frac{m_{3}}{M} \boldsymbol{P}^{\prime}-\frac{m_{3}}{m_{2}+m_{3}} \boldsymbol{q}^{\prime}-\boldsymbol{p}^{\prime}\right)^{2}\right) \\
\simeq \frac{\delta\left(\frac{m_{3}}{M} \Delta P_{\|}-\frac{m_{3}}{m_{2}+m_{3}} \Delta q_{\|}-\Delta p_{\|}+\left(\alpha_{3}-1\right)\left(\frac{m_{3}}{M} P_{\|}-\frac{m_{3}}{m_{2}+m_{3}} q_{\|}-p_{\|}\right)\right)}{2 \alpha_{3}\left(\frac{m_{3}}{M} P_{\|}-\frac{m_{3}}{m_{2}+m_{3}} q_{\|}-p_{\|}\right)}
\end{aligned}
$$

mit

$$
\alpha_{3}:=\sqrt{1+\frac{\left(\frac{m_{3}}{M} P_{\perp}-\frac{m_{3}}{m_{2}+m_{3}} q_{\perp}-p_{\perp}\right)^{2}-\left(-\frac{m_{3}}{m_{2}+m_{3}} q_{\perp}^{\prime}-p_{\perp}^{\prime}\right)^{2}}{\left(\frac{m_{3}}{M} P_{\|}-\frac{m_{3}}{m_{2}+m_{3}} q_{\|}-p_{\|}\right)^{2}}} .
$$

Eine analoge Untersuchung der Impulsverschiebungen zeigt, daß auch hier die Auswertung bei $\alpha_{2}=1$ und $\alpha_{3}=1$ möglich ist. So kann die Trimer-Übergangsamplitude 
(3.78) geschrieben werden als

$$
\begin{aligned}
& t^{(2)}\left(\boldsymbol{P}, \phi_{\gamma} ; \boldsymbol{P}^{\prime}, \phi_{\gamma^{\prime}}\right) \simeq \int \mathrm{d}^{3} q \mathrm{~d}^{3} p \phi_{\gamma}^{*}\left(\boldsymbol{q}, q_{3}, \boldsymbol{p}, p_{3}\right)\{ \\
& \phi_{\gamma^{\prime}}\left(q_{\|}, q_{\perp}-\frac{m_{2}+m_{3}}{M} \Delta P_{\perp}, q_{3}, \boldsymbol{p}, p_{3}\right) \widetilde{t}_{1}^{\mathrm{at}(2)}\left(\frac{m_{1}}{M} \boldsymbol{P}+\boldsymbol{q} ; \Delta \boldsymbol{P}\right) \\
& -2 \pi \mathrm{i} \frac{m_{1}\left(m_{2}+m_{3}\right)}{m_{2}\left(\frac{m_{1}}{M} P_{\|}+q_{\|}\right)} \int \mathrm{d} p_{\perp}^{\prime} \\
& \times \phi_{\gamma^{\prime}}\left(q_{\|}, q_{\perp}-\frac{m_{2}+m_{3}}{m_{2}}\left(\frac{m_{2}}{M} \Delta P_{\perp}+\Delta p_{\perp}\right), q_{3}, p_{\|}, p_{\perp}^{\prime}, p_{3}\right) \\
& \times \widetilde{t}_{1}^{\mathrm{at}(2)}\left(\frac{m_{1}}{M} \boldsymbol{P}+\boldsymbol{q} ; 0, \Delta P_{\perp}+\frac{m_{2}+m_{3}}{m_{2}} \Delta p_{\perp}\right) \\
& \times \widetilde{t}_{3}^{\mathrm{at}(2)}\left(\frac{m_{3}}{M} \boldsymbol{P}-\frac{m_{3}}{m_{2}+m_{3}} \boldsymbol{q}-\boldsymbol{p} ; \Delta P_{\|},-\frac{m_{2}+m_{3}}{m_{2}} \Delta p_{\perp}\right) \\
& -\frac{4 \pi^{2}}{3} \frac{m_{2} m_{3}}{\left(\frac{m_{2}}{M} P_{\|}-\frac{m_{2}}{m_{2}+m_{3}} q_{\|}+p_{\|}\right)\left(\frac{m_{3}}{M} P_{\|}-\frac{m_{3}}{m_{2}+m_{3}} q_{\|}-p_{\|}\right)} \int \mathrm{d} q_{\perp}^{\prime} \mathrm{d} p_{\perp}^{\prime} \\
& \times \phi_{\gamma^{\prime}}\left(q_{\|}, q_{\perp}^{\prime}, q_{3}, p_{\|}, p_{\perp}^{\prime}, p_{3}\right) \\
& \times \widetilde{t}_{1}^{\mathrm{at}(2)}\left(\frac{m_{1}}{M} \boldsymbol{P}+\boldsymbol{q} ; \Delta P_{\|}, \frac{m_{1}}{M} \Delta P_{\perp}+\Delta q_{\perp}\right) \\
& \times{\widetilde{t_{2}}}^{\mathrm{at}(2)}\left(\frac{m_{2}}{M} \boldsymbol{P}-\frac{m_{2}}{m_{2}+m_{3}} \boldsymbol{q}+\boldsymbol{p} ; 0, \frac{m_{2}}{M} \Delta P_{\perp}-\frac{m_{2}}{m_{2}+m_{3}} \Delta q_{\perp}+\Delta p_{\perp}\right) \\
& \left.\times \widetilde{t}_{3}^{\mathrm{at}(2)}\left(\frac{m_{3}}{M} \boldsymbol{P}-\frac{m_{3}}{m_{2}+m_{3}} \boldsymbol{q}-\boldsymbol{p} ; 0, \frac{m_{3}}{M} \Delta P_{\perp}-\frac{m_{3}}{m_{2}+m_{3}} \Delta q_{\perp}-\Delta p_{\perp}\right)\right\} \\
& +\left(\begin{array}{l}
123 \\
231
\end{array}\right)+\left(\begin{array}{l}
123 \\
312
\end{array}\right) \text {. }
\end{aligned}
$$

Die Kleinheit der Relativimpulse (3.66) und die langsame Veränderlichkeit der Einteilchen-Übergangsamplituden erlauben eine weitere Vereinfachung. In den Vorfaktoren sowie im ersten (vektorwertigen) Argument der Funktionen $\widetilde{t}_{i}^{\text {at(2) }}$ dürfen die Relativimpulse gegen den auslaufenden Schwerpunktimpuls $\boldsymbol{P}$ vernachlässigt werden. Mit Hilfe der Substitutionen $\widetilde{q}_{\perp}:=\Delta q_{\perp}$ und $\widetilde{p}_{\perp}:=\Delta p_{\perp}$ können anschließend die Integrationen über $\boldsymbol{q}$ und $\boldsymbol{p}$ formal ausgeführt werden.

$$
\begin{aligned}
& t^{(2)}\left(\boldsymbol{P}, \phi_{\gamma} ; \boldsymbol{P}^{\prime}, \phi_{\gamma^{\prime}}\right) \simeq\{ F_{\gamma \gamma^{\prime}}\left(0,-\frac{m_{2}+m_{3}}{M} \Delta P_{\perp}, 0 ; 0,0,0\right) \widetilde{t}_{1}^{\mathrm{at}(2)}\left(\frac{m_{1}}{M} \boldsymbol{P} ; \Delta \boldsymbol{P}\right) \\
&-2 \pi \mathrm{i} \frac{M\left(m_{2}+m_{3}\right)}{m_{2} P_{\|}} \int \mathrm{d} \widetilde{p}_{\perp} \\
& \quad \times F_{\gamma \gamma^{\prime}}\left(0,-\frac{m_{2}+m_{3}}{m_{2}}\left(\frac{m_{2}}{M} \Delta P_{\perp}+\widetilde{p}_{\perp}\right), 0 ; 0,-\widetilde{p}_{\perp}, 0\right) \\
& \quad \times \widetilde{t}_{1}^{\mathrm{at}(2)}\left(\frac{m_{1}}{M} \boldsymbol{P} ; 0, \Delta P_{\perp}+\frac{m_{2}+m_{3}}{m_{2}} \widetilde{p}_{\perp}\right)
\end{aligned}
$$




$$
\begin{aligned}
& \times \widetilde{t}_{3}^{\mathrm{at}(2)}\left(\frac{m_{3}}{M} \boldsymbol{P} ; \Delta P_{\|},-\frac{m_{2}+m_{3}}{m_{2}} \widetilde{p}_{\perp}\right) \\
-\frac{4 \pi^{2}}{3} \frac{M^{2}}{P_{\|}^{2}} & \int \mathrm{d} \widetilde{q}_{\perp} \mathrm{d} \widetilde{p}_{\perp} F_{\gamma \gamma^{\prime}}\left(0,-\widetilde{q}_{\perp}, 0 ; 0,-\widetilde{p}_{\perp}, 0\right) \\
& \times \widetilde{t}_{1}^{\mathrm{at}(2)}\left(\frac{m_{1}}{M} \boldsymbol{P} ; \Delta P_{\|}, \frac{m_{1}}{M} \Delta P_{\perp}+\widetilde{q}_{\perp}\right) \\
& \times \widetilde{t}_{2}^{\mathrm{at}(2)}\left(\frac{m_{2}}{M} \boldsymbol{P} ; 0, \frac{m_{2}}{M} \Delta P_{\perp}-\frac{m_{2}}{m_{2}+m_{3}} \widetilde{q}_{\perp}+\widetilde{p}_{\perp}\right) \\
& \left.\times \widetilde{t}_{3}^{\mathrm{at}(2)}\left(\frac{m_{3}}{M} \boldsymbol{P} ; 0, \frac{m_{3}}{M} \Delta P_{\perp}-\frac{m_{3}}{m_{2}+m_{3}} \widetilde{q}_{\perp}-\widetilde{p}_{\perp}\right)\right\} \\
+\left(\begin{array}{l}
123 \\
231
\end{array}\right)+ & \left(\begin{array}{l}
123 \\
312
\end{array}\right)
\end{aligned}
$$

Dazu wurde die Funktion

$$
F_{\gamma \gamma^{\prime}}^{(i, j k)}\left(\mathbf{q}^{(i)} ; \mathbf{p}^{(j k)}\right):=\int \mathrm{d}^{3} \widetilde{q} \mathrm{~d}^{3} \widetilde{p}\left(\phi_{\gamma}^{(i, j k)}\right)^{*}(\widetilde{\mathbf{q}}, \widetilde{\mathbf{p}}) \phi_{\gamma^{\prime}}^{(i, j k)}\left(\widetilde{\mathbf{q}}+\mathbf{q}^{(i)}, \widetilde{\mathbf{p}}+\mathbf{p}^{(j k)}\right)
$$

eingeführt, die auch als „molekularer Formfaktor" bezeichnet wird. Wie bisher werden ihre oberen Indizes, die die Realisierung der Jacobi-Koordinaten spezifizieren, nur angegeben, wenn unterschiedliche Realisierungen betrachtet werden müssen.

Gleichung (3.84) stellt die allgemeine Form der Übergangsamplitude eines aus Massen der gleichen Größenordnung bestehenden Trimers an einem in einer Koordinatenrichtung translationsinvarianten Beugungsobjekt unter der Voraussetzung der Beugungsbedingung (2.10) und der großen kinetischen Energie (3.63) dar. Von den speziellen Eigenschaften der Beugung an einem Transmissionsgitter wurde noch nicht Gebrauch gemacht. Dies ist das Thema des folgenden Abschnitts.

\subsubsection{Trimerbeugung am Gitter bei nicht-senkrechtem Ein- fall}

Zur Berechnung der Übergangsamplitude des Trimers an einem Transmissionsgitter müssen die entsprechenden Einteilchen-Übergangsamplituden eingesetzt werden. Da die genauesten verfügbaren experimentellen Daten für Einfallswinkel $\theta^{\prime}>\beta$ vorliegen, wird die folgende Berechnung für diesen Fall durchgeführt. Der senkrechte Einfall ist analog behandelbar, und das zugehörige Ergebnis wird am Ende der Berechnung ebenfalls angegeben.

Die Einteilchen-Übergangsamplitude bei nicht-senkrechtem Einfall für das Transmissionsgitter mit einer ausreichend hohen Anzahl von Gitterstegen $N$ wurde in Kapitel 2.2 berechnet und lautet für das $i$. Teilchen (vgl. Gl. 2.42)

$$
\widetilde{t}_{i, \text { git }}^{\mathrm{at}(2)}\left(\boldsymbol{p}_{i} ; \Delta \boldsymbol{p}_{i}^{\prime}\right) \simeq-\frac{\mathrm{i}}{2} \frac{\pi_{i 1}+\pi_{i 1}^{\prime}}{(2 \pi)^{2} m_{i} \hbar}\left\{2 \pi \hbar \frac{p_{i 1}}{\pi_{i 1}} \delta\left(\Delta p_{i 2}\right)-H_{N}\left(\Delta p_{i 2}\right) a_{i, u}^{\mathrm{at}}\left(\boldsymbol{p}_{i}^{\prime} ; \Delta \pi_{i 2}\right)\right\} .
$$

Analog zu den ebenen Komponenten $\pi_{k}^{\prime}$ und $\pi_{k}$ der Einteilchenimpulse werden im folgenden die Komponenten des einlaufenden und des auslaufenden Schwerpunktimpulsvektors bezüglich des Koordinatensystems $\left(u_{1}, u_{2}\right)$ mit den Symbolen $\Pi_{k}^{\prime}$ beziehungsweise $\Pi_{k}$ bezeichnet. Sie transformieren sich ebenfalls nach Gleichung (2.30). 
Nach dem Einsetzen der Einteilchen-Übergangsamplitude in Gleichung (3.84) und Ausmultiplizieren können einige Integrationen dank der in den Einteilchen-Übergangsamplituden enthaltenen Deltafunktionen bereits ausgeführt werden. Daraufhin ergibt sich der folgende, lange Ausdruck.

$$
\begin{aligned}
& t^{(2)}\left(\boldsymbol{P}, \phi_{\gamma} ; \boldsymbol{P}^{\prime}, \phi_{\gamma^{\prime}}\right) \simeq-\frac{\mathrm{i}}{2} \frac{\Pi_{1}+\Pi_{1}^{\prime}}{(2 \pi)^{2} M \hbar}\{ \\
& F_{\gamma \gamma^{\prime}}\left(0,-\frac{m_{2}+m_{3}}{M} \Delta P_{\perp}, 0 ; 0,0,0\right) \\
& \times\left[2 \pi \hbar \frac{P_{1}}{\Pi_{1}} \delta\left(\Delta P_{2}\right)-H_{N}\left(\Delta P_{2}\right) a_{1, u}^{\mathrm{at}}\left(\boldsymbol{P}^{\prime}-\frac{m_{2}+m_{3}}{M} \boldsymbol{P} ; \Delta \Pi_{2}\right)\right] \\
& -\frac{\Pi_{1}}{P_{\|} \cos \theta^{\prime}}\left[2 \pi \hbar \frac{P_{1}^{2}}{\Pi_{1}^{2}} \delta\left(\Delta P_{2}\right) F_{\gamma \gamma^{\prime}}\left(0,-\frac{m_{2}+m_{3}}{M} \Delta P_{\perp}, 0 ; 0,0,0\right)\right. \\
& -\frac{P_{1}}{\Pi_{1}} F_{\gamma \gamma^{\prime}}\left(0, \frac{m_{1}}{M} \Delta P_{\perp}, 0 ; 0, \frac{m_{2}}{m_{2}+m_{3}} \Delta P_{\perp}, 0\right) H_{N}\left(\Delta P_{2}\right) \\
& \times a_{3, u}^{\text {at }}\left(\boldsymbol{P}_{\|}^{\prime}-\frac{m_{1}+m_{2}}{M} \boldsymbol{P}_{\|} ; \Delta \Pi_{2}\right) \\
& -\frac{P_{1}}{\Pi_{1}} F_{\gamma \gamma^{\prime}}\left(0,-\frac{m_{2}+m_{3}}{M} \Delta P_{\perp}, 0 ; 0,0,0\right) H_{N}\left(\Delta P_{2}\right) \\
& \times a_{1, u}^{\text {at }}\left(\frac{m_{1}}{M} P_{\|}^{\prime}, P_{\perp}^{\prime}-\frac{m_{2}+m_{3}}{M} P_{\perp}-\Delta P_{\|} \tan \theta^{\prime} ; \Delta P_{2} \frac{\cos \phi^{\prime}}{\cos \theta^{\prime}}\right) \\
& +\frac{\cos \theta^{\prime}}{2 \pi \hbar} \frac{m_{2}+m_{3}}{m_{2}} \int \mathrm{d} \widetilde{p}_{\perp} F_{\gamma \gamma^{\prime}}\left(0,-\frac{m_{2}+m_{3}}{m_{2}}\left(\frac{m_{2}}{M} \Delta P_{\perp}+\widetilde{p}_{\perp}\right), 0 ; 0,-\widetilde{p}_{\perp}, 0\right) \\
& \times H_{N}\left(\left(\Delta P_{\perp}+\frac{m_{2}+m_{3}}{m_{2}} \widetilde{p}_{\perp}\right) \cos \theta^{\prime}\right) H_{N}\left(\Delta P_{\|} \sin \theta^{\prime}-\frac{m_{2}+m_{3}}{m_{2}} \widetilde{p}_{\perp} \cos \theta^{\prime}\right) \\
& \times a_{3, u}^{\text {at }}\left(P_{\|}^{\prime}-\frac{m_{1}+m_{2}}{M} P_{\|}, \frac{m_{3}}{M} P_{\perp}+\frac{m_{2}+m_{3}}{m_{2}} \widetilde{p}_{\perp}^{\prime} ;\right. \\
& \left.\Delta P_{\|} \sin \phi^{\prime}-\frac{m_{2}+m_{3}}{m_{2}} \widetilde{p}_{\perp} \cos \phi^{\prime}\right) \\
& \times a_{1, u}^{\mathrm{at}}\left(\frac{m_{1}}{M} P_{\|}, P_{\perp}^{\prime}-\frac{m_{2}+m_{3}}{m_{2}}\left(\frac{m_{2}}{M} P_{\perp}+\widetilde{p}_{\perp}\right) ;\right. \\
& \left.\left.\left(\Delta P_{\perp}+\frac{m_{2}+m_{3}}{m_{2}} \widetilde{p}_{\perp}\right) \cos \phi^{\prime}\right)\right] \\
& +\frac{\Pi_{1}^{2}}{3 P_{\|}^{2} \cos ^{2} \theta^{\prime}}\left[2 \pi \hbar \frac{P_{1}^{3}}{\Pi_{1}^{3}} \delta\left(\Delta P_{2}\right) F_{\gamma \gamma^{\prime}}\left(0-\frac{m_{2}+m_{3}}{M} \Delta P_{\perp}, 0 ; 0,0,0,\right)\right. \\
& -\frac{P_{1}^{2}}{\Pi_{1}^{2}} F_{\gamma \gamma^{\prime}}\left(0, \frac{m_{1}}{M} \Delta P_{\perp}, 0 ; 0, \frac{m_{2}}{m_{2}+m_{3}} \Delta P_{\perp}, 0\right) H_{N}\left(\Delta P_{2}\right) \\
& \times a_{3, u}^{\text {at }}\left(\frac{m_{3}}{M} P_{\|}, P_{\perp}^{\prime}-\frac{m_{1}+m_{2}}{M} P_{\perp} ; \Delta P_{2} \frac{\cos \phi^{\prime}}{\cos \theta^{\prime}}\right)
\end{aligned}
$$




$$
\begin{aligned}
& -\frac{P_{1}^{2}}{\Pi_{1}^{2}} F_{\gamma \gamma^{\prime}}\left(0, \frac{m_{1}}{M} \Delta P_{\perp}, 0 ; 0,-\frac{m_{3}}{m_{2}+m_{3}} \Delta P_{\perp}, 0\right) H_{N}\left(\Delta P_{2}\right) \\
& \times a_{2, u}^{\text {at }}\left(\frac{m_{2}}{M} P_{\|}, P_{\perp}^{\prime}-\frac{m_{3}+m_{1}}{M} P_{\perp} ; \Delta P_{2} \frac{\cos \phi^{\prime}}{\cos \theta^{\prime}}\right) \\
& -\frac{P_{1}^{2}}{\Pi_{1}^{2}} F_{\gamma \gamma^{\prime}}\left(0,-\frac{m_{2}+m_{3}}{M} \Delta P_{\perp}, 0 ; 0,0,0\right) H_{N}\left(\Delta P_{2}\right) \\
& \times a_{1, u}^{\text {at }}\left(\boldsymbol{P}^{\prime}-\frac{m_{2}+m_{3}}{M} \boldsymbol{P} ; \Delta \Pi_{2}\right) \\
& +\frac{\cos \theta^{\prime}}{2 \pi \hbar} \frac{P_{1}}{\Pi_{1}} \frac{m_{1}+m_{2}}{m_{2}} \int \mathrm{d} \widetilde{p}_{\perp}^{*} \\
& F_{\gamma \gamma^{\prime}}\left(0, \frac{m_{1}}{M} \Delta P_{\perp}, 0 ; 0, \frac{m_{2}}{m_{2}+m_{3}} \Delta P_{\perp}+\frac{m_{1}+m_{2}}{m_{1}} \widetilde{p}_{\perp}^{*}, 0\right) \\
& \times H_{N}\left(\left(\Delta P_{\perp}+\frac{m_{1}+m_{2}}{m_{1}} \widetilde{p}_{\perp}^{*}\right) \cos \theta^{\prime}\right) H_{N}\left(\Delta P_{\|} \sin \theta^{\prime}-\frac{m_{1}+m_{2}}{m_{1}} \widetilde{p}_{\perp}^{*} \cos \theta^{\prime}\right) \\
& \times a_{2, u}^{\text {at }}\left(\frac{m_{2}}{M} P_{\|}, \frac{m_{2}}{M} P_{\perp}+\frac{m_{1}+m_{2}}{m_{1}} \widetilde{p}_{\perp}^{*} ;\left(\Delta P_{\|} \tan \theta^{\prime}-\frac{m_{1}+m_{2}}{m_{1}} \widetilde{p}_{\perp}^{*}\right) \cos \phi^{\prime}\right) \\
& \times a_{3, u}^{\mathrm{at}}\left(\frac{m_{3}}{M} P_{\|}, P_{\perp}^{\prime}-\frac{m_{1}+m_{2}}{m_{1}}\left(\frac{m_{1}}{M} P_{\perp}+\widetilde{p}_{\perp}^{*}\right) ;\left(\Delta P_{\perp}+\frac{m_{1}+m_{2}}{m_{1}} \widetilde{p}_{\perp}^{*}\right) \cos \phi^{\prime}\right) \\
& +\frac{\cos \theta^{\prime}}{2 \pi \hbar} \frac{P_{1}}{\Pi_{1}} \frac{m_{2}+m_{3}}{m_{2}} \int \mathrm{d} \widetilde{p}_{\perp}^{*} \\
& F_{\gamma \gamma^{\prime}}\left(0,-\frac{m_{2}+m_{3}}{m_{2}}\left(\frac{m_{2}}{M} \Delta P_{\perp}+\widetilde{p}_{\perp}^{*}\right), 0 ; 0,-\widetilde{p}_{\perp}^{*}, 0\right) \\
& \times H_{N}\left(\left(\Delta P_{\perp}+\frac{m_{2}+m_{3}}{m_{2}} \widetilde{p}_{\perp}^{*}\right) \cos \theta^{\prime}\right) H_{N}\left(\Delta P_{\|} \sin \theta^{\prime}-\frac{m_{2}+m_{3}}{m_{2}} \widetilde{p}_{\perp}^{*} \cos \theta^{\prime}\right) \\
& \times a_{3, u}^{\text {at }}\left(\frac{m_{3}}{M} P_{\|}, \frac{m_{3}}{M} P_{\perp}+\frac{m_{2}+m_{3}}{m_{2}} \widetilde{p}_{\perp}^{*} ;\left(\Delta P_{\|} \tan \theta^{\prime}-\frac{m_{2}+m_{3}}{m_{2}} \widetilde{p}_{\perp}^{*}\right) \cos \phi^{\prime}\right) \\
& \times a_{1, u}^{\text {at }}\left(P_{\|}^{\prime}-\frac{m_{2}+m_{3}}{M} P_{\|}, P_{\perp}^{\prime}-\frac{m_{2}+m_{3}}{m_{2}}\left(\frac{m_{2}}{M} P_{\perp}+\widetilde{p}_{\perp}^{*}\right) ;\right. \\
& \left.\left(\Delta P_{\perp}+\frac{m_{2}+m_{3}}{m_{2}} \widetilde{p}_{\perp}^{*}+\Delta P_{\|}\left(\tan \phi^{\prime}-\tan \theta^{\prime}\right)\right) \cos \phi^{\prime}\right) \\
& +\frac{\cos \theta^{\prime}}{2 \pi \hbar} \frac{P_{1}}{\Pi_{1}} \frac{m_{3}+m_{1}}{m_{3}} \int \mathrm{d} \widetilde{p}_{\perp}^{*} \\
& F_{\gamma \gamma^{\prime}}\left(0, \frac{m_{1}}{M} \Delta P_{\perp}+\frac{m_{3}+m_{1}}{m_{3}} \widetilde{p}_{\perp}^{*}, 0 ; 0,-\frac{m_{3}}{m_{2}+m_{3}} \Delta P_{\perp}-\frac{m_{3}+m_{1}}{m_{2}+m_{3}} \widetilde{p}_{\perp}^{*}, 0\right) \\
& \times H_{N}\left(\left(\Delta P_{\perp}+\frac{m_{3}+m_{1}}{m_{3}} \widetilde{p}_{\perp}^{*}\right) \cos \theta^{\prime}\right) H_{N}\left(\Delta P_{\|} \sin \theta^{\prime}-\frac{m_{3}+m_{1}}{m_{3}} \widetilde{p}_{\perp}^{*} \cos \theta^{\prime}\right) \\
& \times a_{1, u}^{\text {at }}\left(P_{\|}^{\prime}-\frac{m_{2}+m_{3}}{M} P_{\|}, \frac{m_{1}}{M} P_{\perp}+\frac{m_{3}+m_{1}}{m_{3}} \widetilde{p}_{\perp}^{*} ;\right. \\
& \left.\Delta P_{\|} \sin \phi^{\prime}-\frac{m_{3}+m_{1}}{m_{3}} \widetilde{p}_{\perp}^{*} \cos \phi^{\prime}\right)
\end{aligned}
$$




$$
\begin{aligned}
& \times a_{2, u}^{\text {at }}\left(\frac{m_{2}}{M} P_{\|}, P_{\perp}^{\prime}-\frac{m_{3}+m_{1}}{m_{3}}\left(\frac{m_{3}}{M} P_{\perp}+\widetilde{p}_{\perp}^{*}\right) ;\left(\Delta P_{\perp}+\frac{m_{3}+m_{1}}{m_{3}} \widetilde{p}_{\perp}^{*}\right) \cos \phi^{\prime}\right) \\
& -\frac{\cos ^{2} \theta^{\prime}}{(2 \pi \hbar)^{2}} \int \mathrm{d} \widetilde{q}_{\perp} \mathrm{d} \widetilde{p}_{\perp} F_{\gamma \gamma^{\prime}}\left(0,-\widetilde{q}_{\perp}, 0 ; 0,-\widetilde{p}_{\perp}, 0\right) \\
& \times H_{N}\left(\Delta P_{\|} \sin \theta^{\prime}+\left(\frac{m_{1}}{M} \Delta P_{\perp}+\widetilde{q}_{\perp}\right) \cos \theta^{\prime}\right) \\
& \times H_{N}\left(\left(\frac{m_{2}}{M} \Delta P_{\perp}-\frac{m_{2}}{m_{2}+m_{3}} \widetilde{q}_{\perp}^{\prime}+\widetilde{p}_{\perp}\right) \cos \theta^{\prime}\right) \\
& \times H_{N}\left(\left(\frac{m_{3}}{M} \Delta P_{\perp}-\frac{m_{3}}{m_{2}+m_{3}} \widetilde{q}_{\perp}-\widetilde{p}_{\perp}\right) \cos \theta^{\prime}\right) \\
& \times a_{1, u}^{\mathrm{at}}\left(P_{\|}^{\prime}-\frac{m_{2}+m_{3}}{M} P_{\|}, \frac{m_{1}}{M} P_{\perp}^{\prime}-\widetilde{q}_{\perp} ; \Delta P_{\|} \sin \phi^{\prime}+\left(\frac{m_{1}}{M} \Delta P_{\perp}+\widetilde{q}_{\perp}\right) \cos \phi^{\prime}\right) \\
& \times a_{2, u}^{\mathrm{at}}\left(\frac{m_{2}}{M} P_{\|}, \frac{m_{2}}{M} P_{\perp}^{\prime}+\frac{m_{2}}{m_{2}+m_{3}} \widetilde{q}_{\perp}-\widetilde{p}_{\perp} ;\right. \\
& \left.\left(\frac{m_{2}}{M} \Delta P_{\perp}-\frac{m_{2}}{m_{2}+m_{3}} \widetilde{q}_{\perp}+\widetilde{p}_{\perp}\right) \cos \phi^{\prime}\right) \\
& \times a_{3, u}^{\text {at }}\left(\frac{m_{3}}{M} P_{\|}, \frac{m_{3}}{M} P_{\perp}^{\prime}+\frac{m_{3}}{m_{2}+m_{3}} \widetilde{q}_{\perp}+\widetilde{p}_{\perp} ;\right. \\
& \left.\left.\left.\left(\frac{m_{3}}{M} \Delta P_{\perp}-\frac{m_{3}}{m_{2}+m_{3}} \widetilde{q}_{\perp}-\widetilde{p}_{\perp}\right) \cos \phi^{\prime}\right)\right]\right\} \\
& +\left(\begin{array}{l}
123 \\
231
\end{array}\right)+\left(\begin{array}{l}
123 \\
312
\end{array}\right)
\end{aligned}
$$

Dazu wurde in den letzten drei der vier Einfachintegral-Terme die Variable $\widetilde{p}_{\perp}^{\prime}$ nach der Vorschrift (in der Reihenfolge ihres Auftretens von oben nach unten)

$$
\begin{aligned}
\Delta P_{\|} \tan \theta^{\prime}+\frac{m_{1}}{M} \Delta P_{\perp}+\widetilde{q}_{\perp} & =: \Delta P_{\perp}+\frac{m_{2}+m_{3}}{m_{2}} \widetilde{p}_{\perp}^{*} \\
\frac{m_{2}+m_{3}}{M} \Delta P_{\perp}-\widetilde{q}_{\perp} & =: \Delta P_{\perp}+\frac{m_{3}+m_{1}}{m_{3}} \widetilde{p}_{\perp}^{*} \\
\frac{m_{3}}{m_{2}+m_{3}}\left(\Delta P_{\|} \tan \theta^{\prime}+\Delta P_{\perp}\right)-\widetilde{p}_{\perp} & =: \Delta P_{\perp}+\frac{m_{1}+m_{2}}{m_{1}} \widetilde{p}_{\perp}^{*}
\end{aligned}
$$

durch $\widetilde{p}_{\perp}^{*}$ substituiert, so daß die beiden Faktoren $H_{N}$ in den Integranden in allen vier Einfachintegral-Termen dieselben Argumente besitzen (abgesehen von aufsteigenden Permutationen der Teilchenindizes). Außerdem wurden jeweils im ersten (vektorwertigen) Argument aller Spaltamplituden Terme proportional zu $\Delta P_{\|}$vernachlässigt. Analog zur Abschätzung (2.23) dürfen darüberhinaus Impulsüberträge der Form $\Delta \pi_{i 2}=\Delta P_{2} \frac{\cos \phi^{\prime}}{\cos \theta^{\prime}}$ im zweiten Argument der Spaltamplitude durch $\Delta \Pi_{2}$ ersetzt werden.

\section{Einfach- und Zweifachstreuterme}

Die einzelnen Summanden der Trimer-Übergangsamplitude (3.86) enthalten entweder keinen oder einen bis drei Faktoren von Einteilchen-Spaltamplituden. Sie können 
im Rahmen einer Störungsentwicklung des Operators $T_{W}^{(2)}$ als Vorwärtsstreuung beziehungsweise als Einfach-, Zweifach- oder Dreifachstreuung interpretiert werden [40]. Infolgedessen werden sie im folgenden als Vorwärts- beziehungsweise Mehrfachstreuterme bezeichnet.

Zur weiteren Berechnung wird die Trimer-Übergangsamplitude (3.86) nun nach solchen gleichartigen Streutermen sortiert. Von den bestehenden 42 Streutermen werden zunächst beispielhaft jene sechs Einfachstreuterme betrachtet, die die Spaltamplitude des Teilchens mit Index 1 enthalten. Weil dabei Beiträge aus unterschiedlichen Permutationen kombiniert werden, ist es notwendig, die jeweilig verwendete Realisierung der Jacobi-Koordinaten an den Funktionen $F_{\gamma \gamma^{\prime}}$ wieder zu spezifizieren. Der zu untersuchende Anteil der Trimer-Übergangsamplitude lautet

$$
\begin{aligned}
-\frac{\mathrm{i}}{2} & \frac{\Pi_{1}+\Pi_{1}^{\prime}}{(2 \pi)^{2} M \hbar} H_{N}\left(\Delta P_{2}\right) a_{1, u}^{\mathrm{at}}\left(\frac{m_{1}}{M} \boldsymbol{P}^{\prime} ; \Delta \Pi_{2}\right)\{ \\
& {\left[-1+\frac{P_{1}}{P_{\|} \cos \theta^{\prime}}-\frac{1}{3}\left(\frac{P_{1}}{P_{\|} \cos \theta^{\prime}}\right)^{2}\right] F_{\gamma \gamma^{\prime}}^{(1,23)}\left(0,-\frac{m_{2}+m_{3}}{M} \Delta P_{\perp}, 0 ; 0,0,0\right) } \\
+ & {\left[\frac{P_{1}}{P_{\|} \cos \theta^{\prime}}-\frac{1}{3}\left(\frac{P_{1}}{P_{\|} \cos \theta^{\prime}}\right)^{2}\right] F_{\gamma \gamma^{\prime}}^{(2,31)}\left(0, \frac{m_{2}}{M} \Delta P_{\perp}, 0 ; 0, \frac{m_{3}}{m_{3}+m_{1}} \Delta P_{\perp}, 0\right) } \\
+ & {\left.\left[-\frac{1}{3}\left(\frac{P_{1}}{P_{\|} \cos \theta^{\prime}}\right)^{2}\right] F_{\gamma \gamma^{\prime}}^{(3,12)}\left(0, \frac{m_{3}}{M} \Delta P_{\perp}, 0 ; 0,-\frac{m_{2}}{m_{1}+m_{2}} \Delta P_{\perp}, 0\right)\right\} . }
\end{aligned}
$$

Wegen $P_{1} / P_{\|} \cos \theta^{\prime}=1-P_{\perp} \tan \theta^{\prime} / P_{\|} \approx 1$ besitzen die eckigen Klammern hier in guter Näherung die Werte $-\frac{1}{3},+\frac{2}{3}$ und $-\frac{1}{3}$. Dies gibt Anlaß zur Vermutung, daß sich die sechs Summanden von (3.87) näherungsweise gegenseitig aufheben könnten, und der gesamte Ausdruck (3.87) somit vernachlässigbar würde gegenüber anderen Termen der Trimer-Übergangsamplitude (3.86). Dazu müssen allerdings die Funktionswerte der Funktionen $F_{\gamma \gamma^{\prime}}^{(i, j k)}$ übereinstimmen.

\section{Transformationseigenschaft des Formfaktors}

Der Formfaktor (3.85) kann als sechsdimensionale Fourier-Transformation der Bindungswellenfunktionen

$$
\begin{aligned}
F_{\gamma \gamma^{\prime}}^{(i, j k)}\left(\mathbf{q}^{(i)} ; \mathbf{p}^{(j k)}\right):=\int \mathrm{d}^{3} \rho^{(i)} & \mathrm{d}^{3} r^{(j k)} \exp \left[-\mathrm{i}\left(\boldsymbol{\rho}^{(i)} \cdot \mathbf{q}^{(i)}+\mathbf{r}^{(j k)} \cdot \mathbf{p}^{(j k)}\right) / \hbar\right] \\
& \times\left(\phi_{\gamma}^{(i, j k)}\right)^{*}\left(\boldsymbol{\rho}^{(i)}, \mathbf{r}^{(j k)}\right) \phi_{\gamma^{\prime}}^{(i, j k)}\left(\boldsymbol{\rho}^{(i)}, \mathbf{r}^{(j k)}\right)
\end{aligned}
$$

ausgedrückt werden, wobei zur Verdeutlichung die Realisierung der Integrationsvariablen explizit angegeben wurde. Wegen der Invarianz des Skalarprodukts (3.12) unter Wechsel der Realisierung und mit Hilfe der Beziehungen der Bindungswellenfunktionen (3.22) folgt aus (3.88) durch Transformation der Integrationsvariablen auf $\boldsymbol{\rho}^{(j)}, \mathbf{p}^{(k i)}$ unmittelbar die Transformationseigenschaft des Formfaktors bezüglich verschiedener Realisierungen:

$$
F_{\gamma \gamma^{\prime}}^{(i, j k)}\left(\mathbf{q}^{(i)} ; \mathbf{p}^{(j k)}\right)=F_{\gamma \gamma^{\prime}}^{(j, k i)}\left(\mathbf{q}^{(j)} ; \mathbf{p}^{(k i)}\right) .
$$


Angewendet auf den im Ausdruck (3.87) auftretenden Formfaktor $F_{\gamma \gamma^{\prime}}^{(2,31)}$ ergibt sich so

$$
F_{\gamma \gamma^{\prime}}^{(2,31)}(\underbrace{0, \frac{m_{2}}{M} \Delta P_{\perp}, 0}_{\mathbf{q}^{(2)}} ; \underbrace{0, \frac{m_{3}}{m_{3}+m_{1}} \Delta P_{\perp}, 0}_{\mathbf{p}^{(31)}})=F_{\gamma \gamma^{\prime}}^{(1,23)}(\underbrace{0,-\frac{m_{2}+m_{3}}{M} \Delta P_{\perp}}_{\mathbf{q}^{(1)}}, 0 ; \underbrace{0,0,0}_{\mathbf{p}^{(23)}}) .
$$

In den Unterklammerungen sind die Stellvertretervariablen der Funktionen $F_{\gamma \gamma^{\prime}}^{(i, j k)}$ angegeben, um die verwendete Koordinatentransformation (3.5) (hier: $\mathbb{d}^{(13)} \mathbb{d}^{(32)}$ ) zu verdeutlichen. Entsprechend gilt

$$
F_{\gamma \gamma^{\prime}}^{(3,12)}(\underbrace{0, \frac{m_{3}}{M} \Delta P_{\perp}, 0}_{\mathbf{q}^{(3)}} ; \underbrace{0,-\frac{m_{2}}{m_{1}+m_{2}} \Delta P_{\perp}, 0}_{\mathbf{p}^{(12)}})=F_{\gamma \gamma^{\prime}}^{(1,23)}(\underbrace{0,-\frac{m_{2}+m_{3}}{M} \Delta P_{\perp}}_{\mathbf{q}^{(1)}}, 0 ; \underbrace{0,0,0}_{\mathbf{p}^{(23)}})
$$

mit der Transformation $\mathbb{d}^{(13)}$. Die drei in der Summe von Einfachstreutermen des ersten Teilchens (3.87) auftretenden Formfaktoren sind somit identisch und können ausfaktorisiert werden. Die Einfachstreuterme (3.87) lauten daraufhin

$$
\begin{aligned}
& -\frac{\mathrm{i}}{2} \frac{\Pi_{1}+\Pi_{1}^{\prime}}{(2 \pi)^{2} M \hbar}\left[-\left(\frac{P_{\perp}}{P_{\|}} \tan \theta^{\prime}\right)^{2}\right] \\
& \quad \times H_{N}\left(\Delta P_{2}\right) a_{1, u}^{\text {at }}\left(\frac{m_{1}}{M} \boldsymbol{P}^{\prime} ; \Delta \Pi_{2}\right) F_{\gamma \gamma^{\prime}}^{(1,23)}\left(0,-\frac{m_{2}+m_{3}}{M} \Delta P_{\perp}, 0 ; 0,0,0\right) .
\end{aligned}
$$

Der Vorfaktor in eckigen Klammern ist von der Größenordnung $\left(\lambda_{\mathrm{dB}} / s\right)^{2}$. Entsprechendes gilt für die zwei aufsteigenden Permutationen von (3.87), die die Spaltamplitude der Teilchen mit Indizes 2 beziehungsweise 3 enthalten.

Eine ebensolche Untersuchung kann für die vier Zweifachstreuterme der TrimerÜbergangsamplitude (3.86) durchgeführt werden, die jeweils eine Spaltamplitude der Teilchen mit Index 1 und 3 enthalten. Unter Berücksichtigung der Transformationseigenschaft des Formfaktors können auch sie zusammengefaßt werden, und es verbleibt näherungsweise nur der Term

$$
\begin{aligned}
- & \frac{\mathrm{i}}{2} \frac{\Pi_{1}+\Pi_{1}^{\prime}}{(2 \pi)^{2} M \hbar} \frac{1}{2 \pi \hbar} \frac{m_{2}+m_{3}}{m_{2}} \frac{\Pi_{1}}{P_{\|}}\left[-\left(\frac{P_{\perp}}{P_{\|}} \tan \theta^{\prime}\right)\right] \\
& \times \int \mathrm{d} \widetilde{p}_{\perp} F_{\gamma \gamma^{\prime}}^{(1,23)}\left(0,-\frac{m_{2}+m_{3}}{m_{2}}\left(\frac{m_{2}}{M} \Delta P_{\perp}+\widetilde{p}_{\perp}\right), 0 ; 0,-\widetilde{p}_{\perp}, 0\right) \\
& \times H_{N}\left(\left(\Delta P_{\perp}+\frac{m_{2}+m_{3}}{m_{2}} \widetilde{p}_{\perp}\right) \cos \theta^{\prime}\right) H_{N}\left(\Delta P_{\|} \sin \theta^{\prime}-\frac{m_{2}+m_{3}}{m_{2}} \widetilde{p}_{\perp} \cos \theta^{\prime}\right) \\
& \times a_{3, u}^{\text {at }}\left(\frac{m_{3}}{M} P_{\|}^{\prime}, \frac{m_{3}}{M} P_{\perp}+\frac{m_{2}+m_{3}}{m_{2}} \widetilde{p}_{\perp} ; \Delta P_{\|} \sin \phi^{\prime}-\frac{m_{2}+m_{3}}{m_{2}} \widetilde{p}_{\perp} \cos \phi^{\prime}\right) \\
& \times a_{1, u}^{\text {at }}\left(\frac{m_{1}}{M} P_{\|}^{\prime}, P_{\perp}^{\prime}-\frac{m_{2}+m_{3}}{m_{2}}\left(\frac{m_{2}}{M} P_{\perp}+\widetilde{p}_{\perp}\right) ;\left(\Delta P_{\perp}+\frac{m_{2}+m_{3}}{m_{2}} \widetilde{p}_{\perp}\right) \cos \phi^{\prime}\right),
\end{aligned}
$$

dessen Vorfaktor in eckigen Klammern von der Größenordnung $\left(\lambda_{\mathrm{dB}} / s\right)$ ist. Entsprechendes gilt wiederum für die zwei aufsteigenden Permutationen. 


\section{Vorwärts- und Dreifachstreuterme}

Die insgesamt neun Vorwärts- und drei Dreifachstreuterme der Trimer-Übergangsamplitude (3.86) weisen keine Differenzen auf, die zu ihrem Herausheben führen würden. Es wird daher zunächst angenommen, daß die Einfach- (3.90) und Zweifachstreuterme (3.91) gegenüber den Vorwärts- und Dreifachstreutermen vernachlässigt werden können. Daß diese Annahme gerechtfertigt ist, wird nach der expliziten Berechnung des Dreifachstreuterms im folgenden Abschnitt deutlich (s. Seite 57). Nun wieder unter Fortlassung der Realisierungsindizes lautet der verbleibende Ausdruck für die Trimer-Übergangsamplitude

$$
\begin{aligned}
& t^{(2)}(\boldsymbol{P},\left.\phi_{\gamma} ; \boldsymbol{P}^{\prime}, \phi_{\gamma^{\prime}}\right) \simeq-\frac{\mathrm{i}}{2} \frac{\Pi_{1}+\Pi_{1}^{\prime}}{(2 \pi)^{2} M \hbar} \frac{1}{3}\left\{2 \pi \hbar \frac{P_{1}}{\Pi_{1}} \delta\left(\Delta P_{2}\right) \delta_{\gamma \gamma^{\prime}}\right. \\
&- \frac{1}{(2 \pi \hbar)^{2}} \frac{\Pi_{1}^{2}}{P_{\|}^{2}} \int \mathrm{d} \widetilde{q}_{\perp} \mathrm{d} \widetilde{p}_{\perp} F_{\gamma \gamma^{\prime}}\left(0,-\widetilde{q}_{\perp}, 0 ; 0,-\widetilde{p}_{\perp}, 0\right) \\
& \times H_{N}\left(\Delta P_{\|} \sin \theta^{\prime}+\left(\frac{m_{1}}{M} \Delta P_{\perp}+\widetilde{q}_{\perp}\right) \cos \theta^{\prime}\right) \\
& \times H_{N}\left(\left(\frac{m_{2}}{M} \Delta P_{\perp}-\frac{m_{2}}{m_{2}+m_{3}} \widetilde{q}_{\perp}^{\prime}+\widetilde{p}_{\perp}\right) \cos \theta^{\prime}\right) \\
& \times H_{N}\left(\left(\frac{m_{3}}{M} \Delta P_{\perp}-\frac{m_{3}}{m_{2}+m_{3}} \widetilde{q}_{\perp}-\widetilde{p}_{\perp}\right) \cos \theta^{\prime}\right) \\
& \times a_{1, u}^{\mathrm{at}}\left(\frac{m_{1}}{M} \boldsymbol{P}^{\prime} ; \Delta P_{\|} \sin \phi^{\prime}+\left(\frac{m_{1}}{M} \Delta P_{\perp}+\widetilde{q}_{\perp}\right) \cos \phi^{\prime}\right) \\
& \times a_{2, u}^{\mathrm{at}}\left(\frac{m_{2}}{M} \boldsymbol{P}^{\prime} ;\left(\frac{m_{2}}{M} \Delta P_{\perp}-\frac{m_{2}}{m_{2}+m_{3}} \widetilde{q}_{\perp}+\widetilde{p}_{\perp}\right) \cos \phi^{\prime}\right) \\
&\left.\times a_{3, u}^{\mathrm{at}}\left(\frac{m_{3}}{M} \boldsymbol{P}^{\prime} ;\left(\frac{m_{3}}{M} \Delta P_{\perp}-\frac{m_{3}}{m_{2}+m_{3}} \widetilde{q}_{\perp}-\widetilde{p}_{\perp}\right) \cos \phi^{\prime}\right)\right\} \\
&+\left(\begin{array}{l}
123 \\
231
\end{array}\right)+\left(\begin{array}{l}
123 \\
312
\end{array}\right)
\end{aligned}
$$

wobei sich im Vorwärtsstreuterm die Orthogonalität der Bindungswellenfunktionen ausnutzen ließ, die zu $F_{\gamma \gamma^{\prime}}(0 ; 0)=\delta_{\gamma \gamma^{\prime}}$ führt. Außerdem wurde wegen $\Delta P_{\|} \ll P^{\prime}$ in den ersten (vektorwertigen) Argumenten der Einteilchen-Spaltamplituden $P_{\|}$durch $P_{\|}^{\prime}$ ersetzt und die Abhängigkeit von den Relativimpulsen vernachlässigt.

\section{Berechnung des Dreifachstreuterms}

Zur weiteren Berechnung des Dreifachstreuterms wird die Definition der EinteilchenSpaltamplituden (2.40) eingesetzt. Für den Formfaktor wird die Darstellung (3.88) verwendet; vier Integrationen können darin formal ausgeführt werden. Mit der Definition der zweidimensionalen „Ortsraum-Dichte“

$$
\varphi_{\gamma \gamma^{\prime}}^{(i, j k)}\left(\rho_{\perp}, r_{\perp}\right):=\int \mathrm{d} \rho_{\|} \mathrm{d} \rho_{3} \int \mathrm{d} r_{\|} \mathrm{d} r_{3}\left(\phi_{\gamma}^{(i, j k)}\right)^{*}(\boldsymbol{\rho}, \mathbf{r}) \phi_{\gamma^{\prime}}^{(i, j k)}(\boldsymbol{\rho}, \mathbf{r})
$$


lautet er

$$
F_{\gamma \gamma^{\prime}}\left(0,-\widetilde{q}_{\perp}^{\prime}, 0 ; 0,-\widetilde{p}_{\perp}, 0\right)=\int \mathrm{d} \rho_{\perp} \mathrm{d} r_{\perp} \mathrm{e}^{\mathrm{i} \mathrm{q}_{\perp}^{\prime} \rho_{\perp} / \hbar} \mathrm{e}^{\mathrm{i} \widetilde{p}_{\perp}^{\prime} r_{\perp} / \hbar} \varphi_{\gamma \gamma^{\prime}}\left(\rho_{\perp}, r_{\perp}\right) .
$$

Anschließend läßt sich die Integrationsreihenfolge im Dreifachstreuterm vertauschen. Die Integrationen über $\widetilde{q}_{\perp}$ und $\widetilde{p}_{\perp}$ laufen auf die exakt berechenbare Fourier-Transformation des Produkts der drei Gitterfunktionen $H_{N}$ hinaus. Daraufhin lautet der Dreifachstreuterm

$$
\begin{aligned}
& -\frac{\mathrm{i}}{2} \frac{\Pi_{1}+\Pi_{1}^{\prime}}{(2 \pi)^{2} M \hbar} \frac{1}{3}\left(-\frac{\Pi_{1}^{2}}{P_{\|}^{2}}\right) \\
& \times \int_{-\frac{S_{0}}{2}}^{\frac{S_{0}}{2}} \mathrm{~d} u_{2} \tau_{1, u}^{\text {at }}\left(\frac{m_{1}}{M} \boldsymbol{P}^{\prime} ; u_{2}\right) \int_{-\frac{S_{0}}{2}}^{\frac{S_{0}}{2}} \mathrm{~d} u_{2}^{\prime} \tau_{2, u}^{\text {at }}\left(\frac{m_{2}}{M} \boldsymbol{P}^{\prime} ; u_{2}^{\prime}\right) \int_{-\frac{S_{0}}{2}}^{\frac{S_{0}}{2}} \mathrm{~d} u_{2}^{\prime \prime} \tau_{3, u}^{\text {at }}\left(\frac{m_{3}}{M} \boldsymbol{P}^{\prime} ; u_{2}^{\prime \prime}\right) \\
& \times \exp \left(-\mathrm{i} \frac{m_{1} u_{2}+m_{2} u_{2}^{\prime}+m_{3} u_{2}^{\prime \prime}}{M \hbar} \Delta P_{\perp} \cos \phi^{\prime}\right) \exp \left(-\mathrm{i} \frac{\Delta P_{\|} \sin \phi^{\prime} u_{2}}{\hbar}\right) \\
& \times \exp \left(\mathrm{i} \frac{N-1}{2 \hbar} \Delta \Pi_{2} d\right) \sum_{n=0}^{N-1} \sum_{n^{\prime}=0}^{N-1} \sum_{n^{\prime \prime}=0}^{N-1} \exp \left(-\mathrm{i} \frac{m_{1} n^{\prime \prime}+m_{2} n+m_{3} n^{\prime}}{M \hbar} \Delta P_{\perp} \cos \theta^{\prime} d\right) \\
& \times \exp \left(-\mathrm{i} \frac{n^{\prime \prime} \Delta P_{\|} \sin \theta^{\prime} d}{\hbar}\right) \int \mathrm{d} \rho_{\perp} \mathrm{d} r_{\perp} \varphi_{\gamma \gamma^{\prime}}\left(\rho_{\perp}, r_{\perp}\right) \\
& \times \delta\left(\left[n^{\prime \prime}-\frac{n m_{2}+n^{\prime} m_{3}}{m_{2}+m_{3}}\right] \cos \theta^{\prime} d-\left[u_{2}-\frac{m_{2} u_{2}^{\prime}+m_{3} u_{2}^{\prime \prime}}{m_{2}+m_{3}}\right] \cos \phi^{\prime}+\rho_{\perp}\right) \\
& \times \delta\left(\left(n-n^{\prime}\right) \cos \theta^{\prime} d-\left(u_{2}^{\prime}-u_{2}^{\prime \prime}\right) \cos \phi^{\prime}+r_{\perp}\right) .
\end{aligned}
$$

Die Nullstellen des Arguments der Deltafunktion in der letzten Zeile von (3.94) liegen bei

$$
\frac{r_{\perp}}{\cos \phi^{\prime}}=u_{2}^{\prime}-u_{2}^{\prime \prime}-\left(n-n^{\prime}\right) \frac{\cos \theta^{\prime}}{\cos \phi^{\prime}} d .
$$

Es wird nun argumentiert, daß die Beiträge der Terme mit $n \neq n^{\prime}$ vernachlässigt werden dürfen: In den Experimenten von Brühl, Kalinin, Kornilov und Toennies (vgl. Kap. 4) gilt zum Beispiel beim Einfallswinkel von $\theta^{\prime}=18^{\circ}$ typischerweise $d \cos \theta^{\prime} / \cos \phi^{\prime} \approx 3900 \AA$. Die Integrationsvariablen $u_{2}^{\prime}, u_{2}^{\prime \prime}$ sind auf das Intervall $\left[-\frac{S_{0}}{2}, \frac{S_{0}}{2}\right]$ beschränkt, so daß $\left|u_{2}^{\prime}-u_{2}^{\prime \prime}\right| \leq S_{0} \approx 1400 \AA$. Bereits für $\left|n-n^{\prime}\right|=1$ wird die Dichte $\varphi_{\gamma \gamma^{\prime}}\left(\rho_{\perp}, r_{\perp}\right)$ ausgewertet bei $\left|r_{\perp}\right| \gtrsim\left|S_{0} \cos \phi^{\prime}-d \cos \theta^{\prime}\right| \approx 620 \AA$; auf diesem großen Abstand sollte sie aber vernachlässigbar klein sein. An den numerisch berechneten Bindungswellenfunktionen des Helium-Trimers kann die Gültigkeit der Annahme für diesen Spezialfall direkt überprüft werden. Die Nullstellen der anderen Deltafunktion in (3.94) liegen für $n=n^{\prime}$ bei

$$
\frac{\rho_{\perp}}{\cos \phi^{\prime}}=u_{2}-\frac{m_{2} u_{2}^{\prime}+m_{3} u_{2}^{\prime \prime}}{m_{2}+m_{3}}-\left(n^{\prime \prime}-n\right) \frac{\cos \phi^{\prime}}{\cos \theta^{\prime}} d,
$$

und eine entsprechende Argumentation zeigt, daß die Vernachlässigbarkeit der Nullstellen bei $n \neq n^{\prime \prime}$ gerechtfertigt ist. Es werden also im folgenden nur die „Diagonalterme" $n=n^{\prime}=n^{\prime \prime}$ berücksichtigt; sie können als Wechselwirkung aller drei Teilchen 
des Trimers mit demselben Steg des Gitters interpretiert werden. Solche Terme wurden im Kontext der Dimerbeugung in einer Arbeit von Hegerfeldt und Köhler [3] als „kohärenter Anteil der Übergangsamplitude“ bezeichnet. Die Nichtdiagonalterme können hingegen interpretiert werden als Wechselwirkung der einzelnen Teilchen mit unterschiedlichen Stegen des Gitters; sie wurden dort als „inkohärenter Anteil der Übergangsamplitude" bezeichnet.

Aufgrund der Definition der Einteilchen-Transmissionsfunktion (2.32) dürfen die Integrationsgrenzen der Integrale über $u_{2}, u_{2}^{\prime}$ und $u_{2}^{\prime \prime}$ in (3.94) durch unendliche Grenzen ersetzt werden. Daraufhin ist die Ausführung der Integrationen über $u_{2}^{\prime}$ und $u_{2}^{\prime \prime}$ wegen der eben diskutierten Deltafunktionen möglich. Die anschließende Resummation über $n$ liefert wieder eine Gitterfunktion $H_{N}\left(\Delta P_{2}\right)$. Mit der Substitution $U_{2}:=u_{2}-\frac{m_{2}+m_{3}}{M} \frac{\rho_{\perp}}{\cos \phi^{\prime}}$ lautet der Dreifachstreuterm nun

$$
\begin{aligned}
-\frac{\mathrm{i}}{2} \frac{\Pi_{1}+}{(2 \pi)^{2} M \hbar} & \frac{1}{3} \frac{1}{\cos ^{2} \phi^{\prime}}\left(-\frac{\Pi_{1}^{2}}{P_{\|}^{2}}\right) H_{N}\left(\Delta P_{2}\right) \\
\times & \int \mathrm{d} U_{2} \mathrm{e}^{-\mathrm{i} \Delta \Pi_{2} U_{2} / \hbar} \int \mathrm{d} \rho_{\perp}^{(1)} \mathrm{d} r_{\perp}^{(23)} \varphi_{\gamma \gamma^{\prime}}^{(1,23)}\left(\rho_{\perp}^{(1)}, r_{\perp}^{(23)}\right) \\
& \times \tau_{1, u}^{\mathrm{at}}\left(\frac{m_{1}}{M} \boldsymbol{P}^{\prime} ; U_{2}+\frac{m_{2}+m_{3}}{M} \frac{\rho_{\perp}^{(1)}}{\cos \phi^{\prime}}\right) \\
& \times \tau_{2, u}^{\mathrm{at}}\left(\frac{m_{2}}{M} \boldsymbol{P}^{\prime} ; U_{2}-\frac{m_{1}}{M} \frac{\rho_{\perp}^{(1)}}{\cos \phi^{\prime}}+\frac{m_{3}}{m_{2}+m_{3}} \frac{r_{\perp}^{(23)}}{\cos \phi^{\prime}}\right) \\
& \times \tau_{3, u}^{\mathrm{at}}\left(\frac{m_{3}}{M} \boldsymbol{P}^{\prime} ; U_{2}-\frac{m_{1}}{M} \frac{\rho_{\perp}^{(1)}}{\cos \phi^{\prime}}-\frac{m_{2}}{m_{2}+m_{3}} \frac{r_{\perp}^{(23)}}{\cos \phi^{\prime}}\right) \mathrm{e}^{-\mathrm{i} \frac{m_{2}+m_{3}}{M \hbar} \Delta P_{\|} \rho_{\perp}^{(1)} \tan \phi^{\prime}} .
\end{aligned}
$$

Der den Impulsübertrag $\Delta P_{\|}$enthaltende Exponentialfaktor in der letzten Zeile von (3.95) entfällt im Rahmen der bisherigen Näherungen. Darüberhinaus darf der Vorfaktor $\Pi_{1} / P_{\|} \cos \phi^{\prime}=1-P_{\perp} \tan \phi^{\prime} / P_{\|}$durch Eins ersetzt werden. Weiterhin zeigt sich unter Verwendung der Koordinatentransformation (3.2), daß die Ortsargumente der Einteilchen-Transmissionsfunktionen gerade die Projektionen der Teilchenorte $\mathbf{r}_{i}$ auf die geometrische Schattenlinie darstellen, wenn $U_{2} \cos \phi^{\prime}$ als Projektion des Trimer-Schwerpunkts interpretiert wird (vgl. Abschnitt 3.4.4). Es werden daher die Bezeichnungen

$$
\begin{aligned}
& r_{1 \perp}=U_{2} \cos \phi^{\prime}+\frac{m_{2}+m_{3}}{M} \rho_{\perp}^{(1)} \\
& r_{2 \perp}=U_{2} \cos \phi^{\prime}-\frac{m_{1}}{M} \rho_{\perp}^{(1)}+\frac{m_{3}}{m_{2}+m_{3}} r_{\perp}^{(23)} \\
& r_{3 \perp}=U_{2} \cos \phi^{\prime}-\frac{m_{1}}{M} \rho_{\perp}^{(1)}-\frac{m_{2}}{m_{2}+m_{3}} r_{\perp}^{(23)}
\end{aligned}
$$

gewählt. Die Form des Dreifachstreuterms gibt Anlaß zur Definition der TrimerTransmissionsfunktion

$$
\tau_{u, \gamma \gamma^{\prime}}^{\mathrm{tri}}\left(\boldsymbol{P}^{\prime} ; U_{2}\right):=\int \mathrm{d} \rho_{\perp}^{(1)} \mathrm{d} r_{\perp}^{(23)} \varphi_{\gamma \gamma^{\prime}}^{(1,23)}\left(\rho_{\perp}^{(1)}, r_{\perp}^{(23)}\right)
$$




$$
\times \tau_{1, u}^{\mathrm{at}}\left(\frac{m_{1}}{M} \boldsymbol{P}^{\prime} ; \frac{r_{1 \perp}}{\cos \phi^{\prime}}\right) \tau_{2, u}^{\text {at }}\left(\frac{m_{2}}{M} \boldsymbol{P}^{\prime} ; \frac{r_{2 \perp}}{\cos \phi^{\prime}}\right) \tau_{3, u}^{\text {at }}\left(\frac{m_{3}}{M} \boldsymbol{P}^{\prime} ; \frac{r_{3 \perp}}{\cos \phi^{\prime}}\right)
$$

Aufgrund der Transformationseigenschaften (3.22) der Trimer-Bindungswellenfunktionen erfüllt die Dichte die Gleichungen

$$
\varphi_{\gamma \gamma^{\prime}}^{(1,23)}\left(\rho_{\perp}^{(1)}, r_{\perp}^{(23)}\right)=\varphi_{\gamma \gamma^{\prime}}^{(2,31)}\left(\rho_{\perp}^{(2)}, r_{\perp}^{(31)}\right)=\varphi_{\gamma \gamma^{\prime}}^{(3,12)}\left(\rho_{\perp}^{(3)}, r_{\perp}^{(12)}\right)
$$

Die Trimer-Transmissionsfunktion (3.96) ist also erwartungsgemäß unabhängig von der Realisierung der Jacobi-Koordinaten. Weiterhin sind die Einteilchen-Transmissionsfunktionen $\tau_{i, u}^{\text {at }}\left(\mathbf{p}_{i}^{\prime} ; u_{2}\right)$ laut Definition (2.32) nur im Intervall $u_{2} \in\left[-\frac{S_{0}}{2}, \frac{S_{0}}{2}\right]$ von Null verschieden. Diese Eigenschaft überträgt sich auf die Trimer-Transmissionsfunktion (3.96), wie durch Fallunterscheidung gezeigt werden kann. Das ist konsistent mit der Interpretation von $U_{2} \cos \phi^{\prime}$ als Projektion des Trimer-Schwerpunkts. Definiert man analog zur Atombeugung weiterhin eine Trimer-Spaltamplitude durch

$$
a_{u, \gamma \gamma^{\prime}}^{\mathrm{tri}}\left(\boldsymbol{P}^{\prime} ; \Delta \Pi_{2}\right):=\int_{-\frac{S_{0}}{2}}^{\frac{S_{0}}{2}} \mathrm{~d} U_{2} \exp \left(-\mathrm{i} \Delta \Pi_{2} U_{2} / \hbar\right) \tau_{u, \gamma \gamma^{\prime}}^{\mathrm{tri}}\left(\boldsymbol{P}^{\prime} ; U_{2}\right),
$$

so können die zuvor in den drei verschiedenen Realisierungen berechneten Anteile der Trimer-Übergangsamplitude (3.92) wieder zusammengefaßt werden. Sie hat dann die gleiche Struktur wie die Übergangsamplitude der Atombeugung (2.42):

$$
\begin{aligned}
& t^{(2)}\left(\boldsymbol{P}, \phi_{\gamma} ; \boldsymbol{P}^{\prime}, \phi_{\gamma^{\prime}}\right) \simeq \\
& -\frac{\mathrm{i}}{2} \frac{\Pi_{1}+\Pi_{1}^{\prime}}{(2 \pi)^{2} M \hbar}\left\{2 \pi \hbar \frac{P_{1}}{\Pi_{1}} \delta\left(\Delta P_{2}\right) \delta_{\gamma \gamma^{\prime}}-H_{N}\left(\Delta P_{2}\right) a_{u, \gamma \gamma^{\prime}}^{\mathrm{tri}}\left(\boldsymbol{P}^{\prime} ; \Delta \Pi_{2}\right)\right\} .
\end{aligned}
$$

Es bleibt die Möglichkeit der Vernachlässigung der Einfach- und Zweifachstreuterme gegenüber dem Dreifachstreuterm zu überprüfen. Zunächst wird der Dreifachstreuterm mit dem Einfachstreuterm (3.90) verglichen. Bei seiner Berechnung wurde vorausgesetzt, daß die Ortsraum-Dichte $\varphi_{\gamma \gamma^{\prime}}\left(\rho_{\perp}, r_{\perp}\right)$ für Abstände $\rho_{\perp}, r_{\perp}$ im Bereich der (projizierten) Gitterperiode bereits auf vernachlässigbare Werte abgefallen ist. Zur Abschätzung der Größenordnung des Dreifachstreuterms sollte es daher erlaubt sein, die Dichte in der Trimer-Übergangsamplitude (3.96) vorübergehend durch $\delta_{\gamma \gamma^{\prime}} \delta\left(\rho_{\perp}\right) \delta\left(r_{\perp}\right) \mathrm{zu}$ ersetzen. Dies entspricht punktförmigen Trimerzuständen mit der aufsummierten van der Waals-Wechselwirkung der einzelnen Teilchen. Da die van der Waals-Wechselwirkung aber auf geringe Bereiche an den Spalträndern konzentriert ist, kann sie an dieser Stelle gänzlich vernachlässigt werden; in dieser groben Näherung werden die Transmissionsfunktionen, und somit auch die Spaltamplituden, von Atom (2.40) und Trimer (3.98) identisch. Weiterhin kann der Formfaktor im Einfachstreuterm (3.90) betragsmäßig nach oben durch Eins abgeschätzt werden. Dann unterscheidet sich der Einfachstreuterm vom Dreifachstreuterm nur noch durch den Faktor der Größenordnung $\left(\lambda_{\mathrm{dB}} / s\right)^{2}$; er ist also wegen der Beugungsbedingung (2.10) vernachlässigbar. 

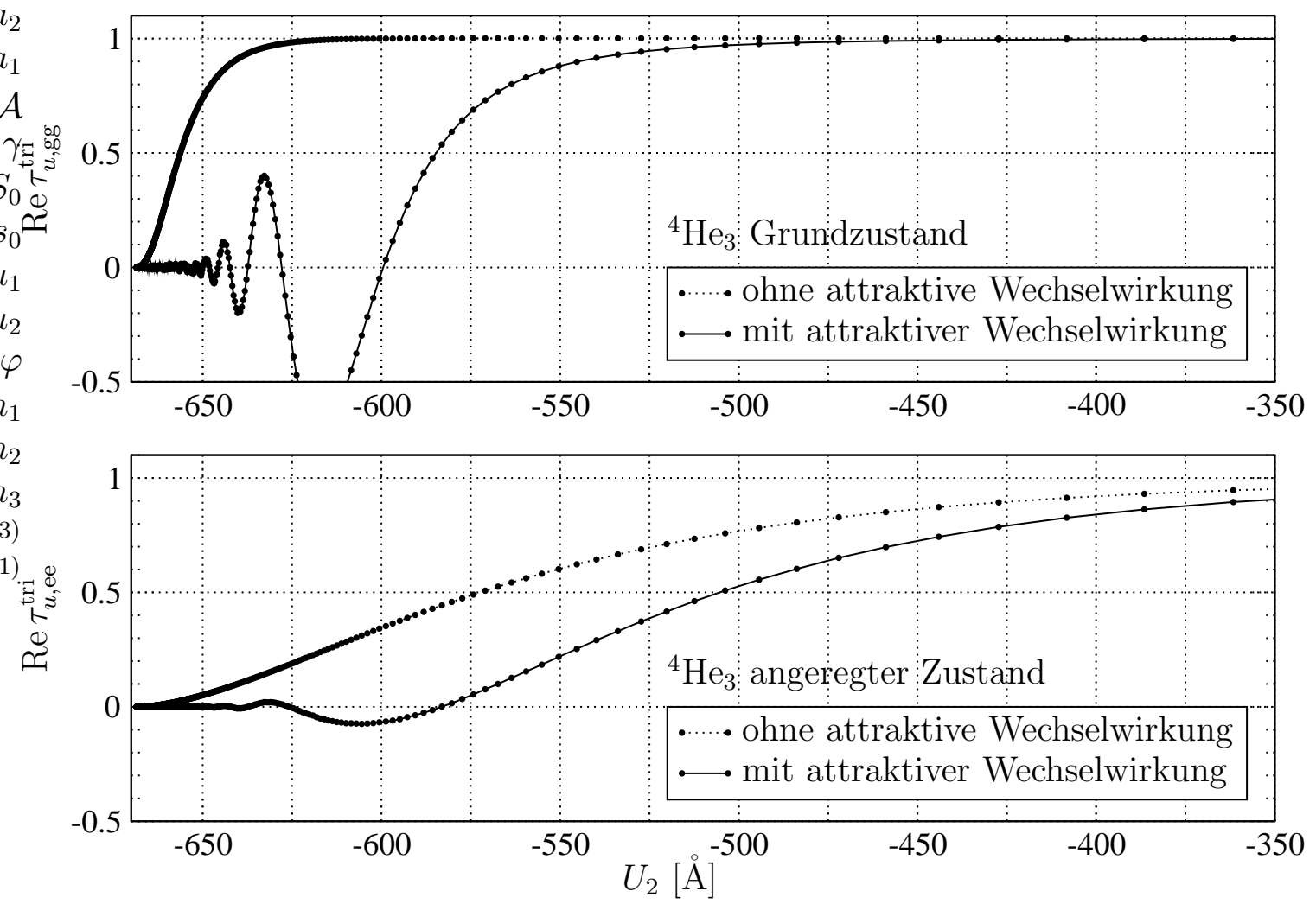

Abbildung 3.3: Ausschnitt aus der numerisch berechneten elastischen Trimer-Transmissionsfunktion (3.96) des Helium-Trimers ${ }^{4} \mathrm{He}_{3}$, aufgetragen über $U_{2}$. Die untere Spaltgrenze befindet sich hier bei $U_{2}=-670 \AA$, die Spaltmitte bei $U_{2}=0$ (nicht im Bild). Die obere Grafik zeigt den Realteil der Transmissionsfunktion des ${ }^{4} \mathrm{He}_{3}$-Grundzustands, die untere den des räumlich wesentlich stärker ausgedehnten, angeregten Zustandes. In beiden Grafiken stellt die dünn gepunktete Kurve die Transmissionsfunktion in Abwesenheit der attraktiven Wechselwirkung dar. Die Abweichung der Transmissionsfunktion dieses hypothetischen Falls von der optischen Transmissionsfunktion (Stufenfunktion) ist auf den Einfluß der endlichen Größe des Trimers zurückzuführen. Die durchgezogenen Kurven zeigen jeweils den experimentell relevanten Fall mit attraktiver Wechselwirkung. In größerer Entfernung vom Spaltrand kommt der Unterschied zwischen den dünn gepunkteten und durchgezogenen Kurven näherungsweise einer Verschiebung der Kurven gegeneinander entlang der $U_{2}$-Achse gleich. Diese Verschiebung entspricht der Reduktion der effektiven Spaltbreite durch den van der Waals-Anteil $S_{\text {eff }, \gamma}^{\mathrm{vdW}}\left(v^{\prime}, \overline{\theta^{\prime}}\right)$ (siehe Kapitel 3.4). Zur numerischen Berechnung wurden $2 M+1$ Stützpunkte $\left(U_{2}\right)_{j}=\frac{S_{0}}{2} \arctan (b j / M) / \arctan (b), j=-M \ldots M$, (fette Punkte) mit $M=500$ und dem Dichteparameter $b=70$ verwendet, um die an den Spalträndern stark oszillierenden Funktionen möglichst genau abzubilden. 
Der Vergleich des Zweifachstreuterms (3.91) mit dem Dreifachstreuterm erfordert etwas mehr Arbeit. Der Zweifachstreuterm kann aber auf einem Wege behandelt werden ähnlich dem, der auch zur einfachen Darstellung des Dreifachstreuterms führte, und schließlich wieder auf die Form eines Produkts aus Gitterfunktion und Spaltamplitude gebracht werden. Mit Hilfe derselben Argumentation wie beim Einfachstreuterm folgt, daß der Zweifachstreuterm sich wesentlich nur durch einen Vorfaktor der Größenordnung $\left(\lambda_{\mathrm{dB}} / s\right)$ vom Dreifachstreuterm unterscheidet. Er ist somit ebenfalls vernachlässigbar.

\subsection{Das Beugungsbild des Trimers}

Die Übergangsamplitude des Trimers bestimmt das Streumatrixelement und somit das Beugungsbild. Aufgrund ihrer formalen Ähnlichkeit mit der Übergangsamplitude der Atombeugung kann das Beugungsbild des Trimers analog berechnet werden. Wegen der Möglichkeit der An- oder Abregung sowie des Aufbruchs ist das Beugungsbild des Trimers jedoch reicher als das des einzelnen Atoms. Anschließend wird ein Zusammenhang zwischen der effektiven Spaltbreite des Trimers und der räumlichen Ausdehnung des Trimers abgeleitet. Dieser Zusammenhang läßt sich physikalisch anschaulich interpretieren und ist die wichtigste Voraussetzung zur quantitativen Auswertung von Beugungsexperimenten.

\subsection{1 Übertragung von Ergebnissen der Atombeugung}

Die Übergangsamplituden des Atoms (2.42) und des Trimers (3.99) besitzen formal die gleiche Struktur. Sie unterscheiden sich nur in den jeweiligen Transmissionsfunktionen (2.32) beziehungsweise (3.96). Aus diesem Grund kann die Berechnung der Beugungsintensitäten mit geringen Erweiterungen aus Abschnitt 2.2.3 übernommen werden.

Die Wahrscheinlichkeitsdichte dafür, ein Trimer mit auslaufenden Schwerpunktimpuls $\mathbf{P}$ im Bindungszustand $\left|\phi_{\gamma}\right\rangle$ zu messen, wurde in Abschnitt 3.2.1 berechnet. Laut Gleichung (3.46) ist sie gegeben durch

$$
w\left(\mathbf{P}, \phi_{\gamma} ; \rho\right)=\sum_{\gamma^{\prime}} p_{\gamma^{\prime}}\left|\left\langle\mathbf{P}, \phi_{\gamma}\left|S_{V V}\right| \psi_{\text {in }}, \phi_{\gamma^{\prime}}\right\rangle\right|^{2},
$$

wobei $p_{\gamma^{\prime}}$ die relative Häufigkeit des Bindungszustands $\left|\phi_{\gamma^{\prime}}\right\rangle$ im einlaufenden Strahl angibt. Das gesamte Beugungsbild entsteht aus der inkohärenten Überlagerung einzelner Beugungsprozesse, die von den unterschiedlichen einlaufenden Bindungszuständen $\left|\phi_{\gamma^{\prime}}\right\rangle$ herrühren. Es genügt, einen dieser Beugungsprozesse zu betrachten. Die Kombination der Gleichungen für das Element der Streumatrix (3.59) und für die Übergangsamplitude des Trimers (3.99) unter Berücksichtigung der Translationsinvarianz entlang der dritten Koordinatenrichtung (3.61) liefert

$$
\left|\left\langle\mathbf{P}, \phi_{\gamma}\left|S_{V V}\right| \psi_{\text {in }}, \phi_{\gamma^{\prime}}\right\rangle\right|^{2}=\mid \int \mathrm{d}^{3} P^{\prime} \psi\left(\mathbf{P}^{\prime}\right)\left[\delta^{(3)}(\Delta \mathbf{P}) \delta_{\gamma \gamma^{\prime}}\right.
$$




$$
\left.-\frac{\Pi_{1}+\Pi_{1}^{\prime}}{4 \pi M \hbar} \delta\left(\Delta P_{3}\right) \delta\left(E-E^{\prime}\right)\left\{2 \pi \hbar \frac{P_{1}}{\Pi_{1}} \delta\left(\Delta P_{2}\right) \delta_{\gamma \gamma^{\prime}}-H_{N}\left(\Delta P_{2}\right) a_{u, \gamma \gamma^{\prime}}^{\mathrm{tri}}\left(\boldsymbol{P}^{\prime} ; \Delta \Pi_{2}\right)\right\}\right]\left.\right|^{2} .
$$

Dies entspricht im wesentlichen Gleichung (2.44); die Energien sind hier allerdings durch $E=|\mathbf{P}|^{2} / 2 M+E_{\gamma}$ beziehungsweise $E^{\prime}=\left|\mathbf{P}^{\prime}\right|^{2} / 2 M+E_{\gamma^{\prime}}$ (vgl. Gl. 3.16) gegeben. Analog zu dem Vorgehen bei der Atombeugung in Abschnitt 2.2.3 läßt sich zeigen, daß sich die beiden ersten Summanden in den eckigen Klammern von Gleichung (3.100) kompensieren. Ersetzt man nun die Definition (2.48) der Funktion $\alpha\left(p_{\perp}^{\prime}\right)$ im Fall der Trimerbeugung durch die erweiterte Definition

$$
1-\alpha^{\gamma \gamma^{\prime}}\left(P_{\perp}^{\prime}\right):=\sqrt{1+\frac{P_{\perp}^{2}-P_{\perp}^{\prime 2}+2 M\left(E_{\gamma}-E_{\gamma^{\prime}}\right)}{P_{\|}^{2}}}
$$

und jene der Nullstellen (2.52) durch

$$
P_{\perp}^{\prime \gamma \gamma^{\prime}\left(n^{\prime}\right)}:=-\sin \overline{\theta^{\prime}} \sqrt{|\boldsymbol{P}|^{2}+2 M\left(E_{\gamma}-E_{\gamma^{\prime}}\right)-\left[P_{2}-\frac{n^{\prime} 2 \pi \hbar}{d}\right]^{2}}+\cos \overline{\theta^{\prime}}\left[P_{2}-\frac{n^{\prime} 2 \pi \hbar}{d}\right],
$$

so kann die weitere Ableitung direkt übernommen werden. Entsprechend Gleichung (2.56) folgt, daß die $n$. Beugungsordnung unter dem auslaufenden Impuls

$$
P_{2}=\bar{P}_{2}^{\prime}+\frac{n 2 \pi \hbar}{d}
$$

zu finden ist, wobei $\overline{\mathbf{P}}^{\prime}$ den mittleren einlaufenden Impuls des Trimerstrahls bezeichnet.

Aufgrund der erweiterten Energieerhaltung, die inelastische Streuprozesse erlaubt, weichen die zu (3.101) gehörenden Beugungswinkel $\theta_{n}$ allerdings im allgemeinen von jenen der Atombeugung (2.57) ab. Wegen $\sin \theta_{n}=P_{2} /|\boldsymbol{P}|$ und $\sin \overline{\theta^{\prime}}=$ $\bar{P}_{2}^{\prime} /\left|\overline{\boldsymbol{P}}^{\prime}\right|$ gilt für den Winkel der $n$. Beugungsordnung des Streuprozesses $\gamma^{\prime} \rightarrow \gamma$

$$
\sin \left(\theta_{n}^{\gamma \gamma^{\prime}}\right)=\frac{\left|\overline{\boldsymbol{P}}^{\prime}\right|}{|\boldsymbol{P}|}\left[\sin \overline{\theta^{\prime}}+\frac{n 2 \pi \hbar}{\left|\overline{\boldsymbol{P}}^{\prime}\right| d}\right]
$$

und der Vorfaktor kann geschrieben werden als

$$
\frac{\left|\overline{\boldsymbol{P}}^{\prime}\right|}{|\boldsymbol{P}|}=\left[1-\frac{E_{\gamma}-E_{\gamma^{\prime}}}{\left|\overline{\boldsymbol{P}}^{\prime}\right|^{2} / 2 M}\right]^{-\frac{1}{2}} .
$$

Im Falle elastischer $\left(\gamma=\gamma^{\prime}\right)$ Beugung ist $\theta_{n}^{\gamma \gamma} \equiv \theta_{n}$ unabhängig vom Bindungszustand des Trimers, und Gleichung (3.102) geht über in ihr Pendant bei der Atombeugung (2.57). Im Falle der inneren Anregung $\left(E_{\gamma}>E_{\gamma^{\prime}}\right)$ bewirkt der Vorfaktor $\left|\overline{\boldsymbol{P}}^{\prime}\right| /|\boldsymbol{P}|>1$ eine Streckung des Beugungsbildes sowie eine Verschiebung der nullten Beugungsordnung zu $\theta_{0}^{\gamma \gamma^{\prime}}>\theta^{\prime}$; im Falle der Abregung $\left(E_{\gamma}<E_{\gamma^{\prime}}\right)$ hingegen staucht der Vorfaktor $\left|\overline{\boldsymbol{P}}^{\prime}\right| /|\boldsymbol{P}|<1$ das Beugungsbild und verschiebt die nullte Beugungsordnung zu einem 
Beugungswinkel $\theta_{0}^{\gamma \gamma^{\prime}}<\theta^{\prime}$. Für die Intensitäten der Beugungsmaxima des Prozesses $\gamma^{\prime} \rightarrow \gamma$ erhält man, entsprechend Gleichung (2.58),

$$
I_{n}^{\gamma \gamma^{\prime}} \propto\left(\frac{\Pi_{1}+\bar{\Pi}_{1}^{\prime}}{2\left|\overline{\boldsymbol{P}}^{\prime}\right| d \cos \overline{\theta^{\prime}}}\right)^{2}\left|a_{u, \gamma \gamma^{\prime}}^{\mathrm{tri}}\left(\overline{\boldsymbol{P}}^{\prime} ; \Delta \Pi_{2}\right)\right|^{2} .
$$

Die Komponenten $\bar{\Pi}_{1}^{\prime}, \bar{\Pi}_{2}^{\prime}$ sind beim mittleren einlaufenden Schwerpunktimpuls $\overline{\boldsymbol{P}}^{\prime}$ auszuwerten, die Komponenten $\Pi_{1}, \Pi_{2}$ beim auslaufenden Impuls $\boldsymbol{P}$ des Prozesses $\gamma^{\prime} \rightarrow \gamma$. Das gesamte Beugungsbild setzt sich nach Gleichung (3.46) additiv aus den gewichteten Intensitäten $p_{\gamma^{\prime}} I_{n}^{\gamma \gamma^{\prime}}$ bei den jeweiligen Beugungswinkeln $\theta_{n}^{\gamma \gamma^{\prime}}$ zusammen.

Es bleibt anzumerken, daß die Gleichungen (3.102) bis (3.104) nicht auf den Fall der Trimerbeugung beschränkt sind. Ihre Ableitung ist für jeden kollimierten Strahl von schwach gebundenen Clustern möglich, solange sich die Übergangsamplitude in der Form (3.99) mit einer entsprechenden Spaltamplitude darstellen läßt. Die Gleichungen gelten insbesondere auch für die Dimerbeugung; davon wird im Kapitel 5 Gebrauch gemacht. Darüberhinaus vereinfachen sie sich für $E_{\gamma}=0$ und $\gamma=\gamma^{\prime}$ formal wieder auf den bekannten Fall der Atombeugung.

Ebenfalls in Analogie zum Vorgehen in der Atombeugung auf den Seiten 18ff kann eine Kumulantenentwicklung der Trimer-Spaltamplitude (3.98) durchgeführt werden. Dies ist allerdings nur für elastische Beugungsprozesse sinnvoll, da die Trimer-Transmissionsfunktion nur in diesem Fall Ähnlichkeit mit einer optischen (oder atomaren) Transmissionsfunktion hat. Bei inelastischer Beugung hingegen verschwindet die Trimer-Transmissionsfunktion in der Spaltmitte und wächst zu den Spalträndern hin an; dies ist konsistent mit der anschaulichen Vorstellung, daß die An- oder Abregung räumlich in der Nähe der Stegoberflächen stattfindet, nicht aber in der Mitte des Spalts.

Den unterhalb von Gleichung (3.94) durchgeführten Überlegungen zufolge erfüllt die elastische Trimer-Transmissionsfunktion auch in der Spaltmitte $\tau_{u, \gamma \gamma}^{\text {tri }}\left(\boldsymbol{P}^{\prime} ; 0\right) \simeq 1$ (vgl. Abb. 3.3). Die Kumulantenentwicklung der atomaren Spaltamplitude (2.65) kann daher ohne erneute Berechnung auf die elastische Trimer-Spaltamplitude (3.98) übertragen werden. Die ersten beiden Kumulanten für die elastische Beugung des Trimers im Bindungszustand $\left|\phi_{\gamma}\right\rangle$ lauten, entsprechend den Gleichungen (2.62) und (2.63),

$$
R_{1, \gamma}^{ \pm}=\frac{S_{0}}{2}-\int_{0}^{S_{0} / 2} \mathrm{~d} \xi \tau_{u, \gamma \gamma}^{\mathrm{tri}}\left(\boldsymbol{P}^{\prime} ; \pm\left(\frac{S_{0}}{2}-\xi\right)\right)
$$

und

$$
R_{2, \gamma}^{ \pm}=\left(\frac{S_{0}}{2}\right)^{2}-\left(R_{1, \gamma}^{ \pm}\right)^{2}-2 \int_{0}^{S_{0} / 2} \mathrm{~d} \xi \xi \tau_{u, \gamma \gamma}^{\mathrm{tri}}\left(\boldsymbol{P}^{\prime} ; \pm\left(\frac{S_{0}}{2}-\xi\right)\right)
$$

Wiederum können Funktionen

$$
\begin{aligned}
S_{\mathrm{eff}, \gamma} & :=S_{0}-\operatorname{Re}\left(R_{1, \gamma}^{+}+R_{1, \gamma}^{-}\right) \\
\Delta_{\gamma} & :=\operatorname{Im}\left(R_{1, \gamma}^{+}+R_{1, \gamma}^{-}\right) \\
\alpha_{\gamma} & :=\operatorname{Im}\left(R_{1, \gamma}^{+}-R_{1, \gamma}^{-}\right)
\end{aligned}
$$




$$
\begin{aligned}
\Sigma_{\gamma} & :=\sqrt{\frac{1}{2} \operatorname{Re}\left(R_{2, \gamma}^{+}+R_{2, \gamma}^{-}\right)} \\
\Omega_{R, \gamma} & :=\frac{1}{2} \operatorname{Re}\left(R_{2, \gamma}^{+}-R_{2, \gamma}^{-}\right) ; \quad \Omega_{I, \gamma}:=\frac{1}{2} \operatorname{Im}\left(R_{2, \gamma}^{+}-R_{2, \gamma}^{-}\right)
\end{aligned}
$$

und der für elastische Beugung vom Bindungszustand unabhängige Wellenzahlübertrag

$$
K\left(\theta_{n}\right):=\frac{\Delta \Pi_{2}}{\hbar}=\frac{\left|\boldsymbol{P}^{\prime}\right|}{\hbar}\left(\cos \left(\varphi-\theta_{n}\right)-\cos \left(\varphi-\overline{\theta^{\prime}}\right)\right)
$$

definiert werden. Bei der Atombeugung war es möglich, in Gleichung (2.74) die $\Omega_{R}$ und $\Omega_{I}$ enthaltenden Terme zu vernachlässigen. Bei der Trimerbeugung ist diese Näherung etwas weniger zuverlässig, wie ein numerischer Vergleich zeigt ${ }^{3}$; für eine quantitativ genaue Formel sollte der Term $\Omega_{R, \gamma}$ beibehalten werden. Somit erhält man die kumulantenentwickelte Intensitätsformel für elastische Beugungsprozesse

$$
\begin{aligned}
I_{n}^{\gamma \gamma} \propto & \left(\frac{\sin \left(\varphi-\theta_{n}\right)+\sin \left(\varphi-\overline{\theta^{\prime}}\right)}{2 \cos \overline{\theta^{\prime}}}\right)^{2} \exp \left[-\alpha_{\gamma} K\left(\theta_{n}\right)\right] \exp \left[-\Sigma_{\gamma}^{2} K\left(\theta_{n}\right)^{2}\right] \\
& \times \frac{\sin ^{2}\left[K\left(\theta_{n}\right) S_{\mathrm{eff}, \gamma} / 2\right]+\sinh ^{2}\left[K\left(\theta_{n}\right) \Delta_{\gamma} / 2+K^{2}\left(\theta_{n}\right) \Omega_{R, \gamma} / 2\right]}{\left[K\left(\theta_{n}\right) d / 2\right]^{2}}
\end{aligned}
$$

die ebenfalls die Interpretation der Größe $S_{\text {eff, } \gamma}$ als „effektive Spaltbreite“ erlaubt. In Abbildung 3.4 werden die Intensitätsformeln vor (3.104) und nach (3.113) der Kumulantenentwicklung miteinander verglichen.

\subsubsection{Die räumliche Ausdehnung der Bindungszustände}

In der Atombeugung besteht die Nützlichkeit der Kumulantenentwicklung darin, daß sie bereits in erster Näherung einen Zusammenhang zwischen der attraktiven Wechselwirkung und der effektiven Spaltbreite des Transmissionsgitters liefert. Im Limes verschwindender attraktiver Wechselwirkung gehen die atomaren Kumulanten $R_{1}^{ \pm}, R_{2}^{ \pm}$gegen Null und somit $S_{\text {eff }}$ gegen $S_{0}$. Da die Kumulanten der Trimerbeugung $R_{1, \gamma}^{ \pm}, R_{2, \gamma}^{ \pm}$formal die gleiche Struktur besitzen wie jene der Atombeugung, liegt eine entsprechende Untersuchung nahe.

\section{Die effektive Spaltbreite der Trimerbeugung}

Für die effektive Spaltbreite der Trimerbeugung (3.107) erhält man mit Hilfe der Ausdrücke für die Kumulanten (3.105) die Darstellung

$$
S_{\mathrm{eff}, \gamma}\left(v^{\prime}, \overline{\theta^{\prime}}\right)=S_{0}-\operatorname{Re} \int_{-S_{0} / 2}^{S_{0} / 2} \mathrm{~d} U_{2}\left[1-\tau_{u, \gamma \gamma}^{\mathrm{tri}}\left(\overline{\boldsymbol{P}}^{\prime} ; U_{2}\right)\right], \quad v^{\prime}=\frac{\left|\overline{\boldsymbol{P}}^{\prime}\right|}{M} .
$$

\footnotetext{
${ }^{3}$ Bei den zu Abb. 3.4 gehörenden Strahlparametern $v^{\prime}=484,1 \mathrm{~m} / \mathrm{s}$ und $\theta^{\prime}=21^{\circ}$ sowie den Gitterparametern $\varphi=0,56197 \mathrm{rad}, S_{0}=1397,5 \AA, \beta=6,7^{\circ}$ und $C_{3}=0,155 \mathrm{meV} \mathrm{nm}^{3}$ für $n=7$ findet man für den Grundzustand des Trimers beispielsweise folgende Werte: $K\left(\theta_{7}\right) S_{\text {eff, } \gamma} / 2=5,38$ und $K\left(\theta_{7}\right)^{2} \Omega_{I, \gamma} / 2=0,04$; sowie $K\left(\theta_{7}\right) \Delta_{\gamma} / 2=-0,82$ und $K\left(\theta_{7}\right)^{2} \Omega_{R, \gamma} / 2=-0,09$.
} 


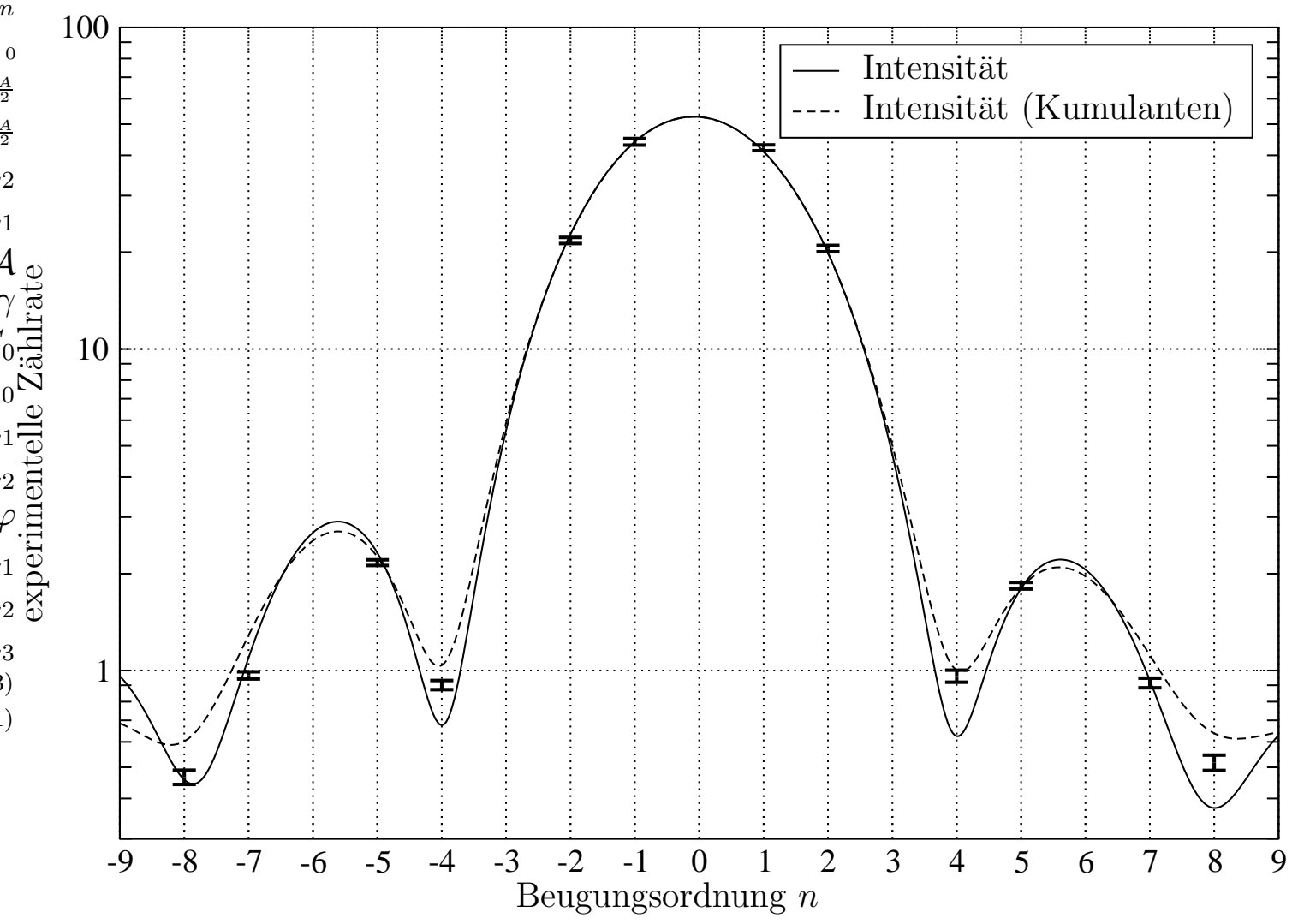

Abbildung 3.4: Vergleich der Intensitätsformel (Gl. 3.104, durchgezogene Kurve) mit der durch zwei Kumulanten approximierten Formel (Gl. 3.113, gestrichelte Kurve) am Beispiel der elastischen Beugung des Grundzustandes von ${ }^{4} \mathrm{He}_{3}$ unter Verwendung der numerisch berechneten Bindungswellenfunktion (vgl. Anh. B). Zur Veranschaulichung wird die Spaltamplitude kontinuierlich dargestellt; experimentell relevant sind aber nur die Werte bei ganzzahligen Beugungsordnungen $n$. Die geometrischen Parameter für das Gitter und die attraktive Wechselwirkung wurden der Auswertung der Beugungsexperimente von Brühl, Kalinin, Kornilov und Toennies in Kapitel 4 entnommen, ebenso die zum Vergleich eingezeichneten experimentellen Beugungsintensitäten aus der Messung $\mathrm{zu} \theta^{\prime}=21^{\circ}$ und $v^{\prime}=484,1 \mathrm{~ms}^{-1}$. Beide theoretischen Kurven sind um denselben Faktor auf die experimentelle Zählrate skaliert. 
Sie unterscheidet sich demnach von der geometrischen Spaltbreite $S_{0}$ durch die über den Spalt aufsummierte Abweichung der Trimer-Transmissionsfunktion von der optischen Transmissionsfunktion. In die Trimer-Transmissionsfunktion (3.96) gehen sowohl die attraktive Wechselwirkung als auch der Bindungszustand des Trimers ein: Verschwindet erstere, so bleibt dennoch ein Größeneffekt (vgl. Abb. 3.3). Ist hingegen die räumliche Ausdehnung des Trimers sehr gering, so geht die Trimer-Transmissionsfunktion in die atomare Transmissionsfunktion mit aufsummierter attraktiver Wechselwirkung über. Die folgenden Überlegungen sollen zeigen, ob die Trennung dieser beiden Einflüsse möglich ist.

Wenn nicht anders erwähnt, wird wieder die Realisierung $\boldsymbol{\rho} \equiv \boldsymbol{\rho}^{(1)}, \mathbf{r}=\mathbf{r}^{(23)}$ der Jacobi-Koordinaten verwendet. Einsetzen der Trimer-Transmissionsfunktion (3.96) in die effektive Spaltbreite (3.114) und Vertauschen der Integrationsreihenfolge führt auf die Gleichung

$$
\begin{aligned}
& S_{\text {eff }, \gamma}\left(v^{\prime}, \overline{\theta^{\prime}}\right)=S_{0}-\operatorname{Re} \int_{-\infty}^{\infty} \mathrm{d} \rho_{\perp} \int_{-\infty}^{\infty} \mathrm{d} r_{\perp} \varphi_{\gamma \gamma}\left(\rho_{\perp}, r_{\perp}\right) \int_{-S_{0} / 2}^{S_{0} / 2} \mathrm{~d} U_{2} \\
& \times[1-\tau_{1, u}^{\mathrm{at}}\left(\frac{m_{1}}{M} \overline{\boldsymbol{P}}^{\prime} ; U_{2}+\frac{m_{2}+m_{3}}{M} \frac{\rho_{\perp}}{\cos \phi^{\prime}}\right) \\
& \times \tau_{2, u}^{\mathrm{at}}\left(\frac{m_{2}}{M} \overline{\boldsymbol{P}}^{\prime} ; U_{2}-\frac{m_{1}}{M} \frac{\rho_{\perp}}{\cos \phi^{\prime}}+\frac{m_{3}}{m_{2}+m_{3}} \frac{r_{\perp}}{\cos \phi^{\prime}}\right) \\
&\left.\times \tau_{3, u}^{\mathrm{at}}\left(\frac{m_{3}}{M} \overline{\boldsymbol{P}}^{\prime} ; U_{2}-\frac{m_{1}}{M} \frac{\rho_{\perp}}{\cos \phi^{\prime}}-\frac{m_{2}}{m_{2}+m_{3}} \frac{r_{\perp}}{\cos \phi^{\prime}}\right)\right] .
\end{aligned}
$$

Ihrer Definition (2.32) zufolge verschwinden die drei atomaren Transmissionsfunktionen, wenn sich ihre Ortsargumente außerhalb des Intervalls $\left[-\frac{S_{0}}{2}, \frac{S_{0}}{2}\right]$ befinden. Diese Eigenschaft erlaubt es, in Gleichung (3.115) das Integrationsintervall von $U_{2}$ zu reduzieren. Dazu ist eine Fallunterscheidung notwendig: im folgenden wird das Doppelintegral über $r_{\perp}$ und $U_{2}$ in Gleichung (3.115) auf den durch die vier möglichen Kombinationen von $r_{\perp} \lessgtr 0$ und $U_{2} \lessgtr 0$ bestimmten Quadranten gesondert betrachtet.

Zunächst wird der durch $r_{\perp}>0, U_{2}>0$ bestimmte Quadrant untersucht. Die Ortsargumente der drei atomaren Transmissionsfunktionen sind kleiner als die obere Spaltgrenze $S_{0} / 2$, falls

$U_{2}<\frac{S_{0}}{2}-\frac{1}{\cos \phi^{\prime}} \max \left\{\frac{m_{2}+m_{3}}{M} \rho_{\perp},-\frac{m_{1}}{M} \rho_{\perp}+\frac{m_{3}}{m_{2}+m_{3}} r_{\perp},-\frac{m_{1}}{M} \rho_{\perp}-\frac{m_{2}}{m_{2}+m_{3}} r_{\perp}\right\}$,

wobei die Funktion max den Maximalwert ihrer Argumente liefert. Wegen $r_{\perp}>0$ ist das dritte Argument in (3.116) hier kleiner als das zweite und braucht nicht berücksichtigt zu werden. Mit Hilfe der Identität

$$
\max \{a, b\}=\frac{1}{2}\{a+b+|a-b|\}
$$

und der Definition

$$
\Delta_{1}^{ \pm}:= \pm \frac{1}{2}\left\{\frac{m_{2}+m_{3}-m_{1}}{M} \rho_{\perp}+\frac{m_{3}}{m_{2}+m_{3}} r_{\perp} \pm\left|\rho_{\perp}-\frac{m_{3}}{m_{2}+m_{3}} r_{\perp}\right|\right\}
$$


nimmt die Bedingung an die obere Integrationsgrenze (3.116) die einfache Form

$$
U_{2}<\frac{S_{0}}{2}-\frac{\Delta_{1}^{+}}{\cos \phi^{\prime}}
$$

an. Entsprechend läßt sich der Quadrant $r_{\perp}>0, U_{2}<0$ untersuchen. Die Bedingung dafür, daß die Ortsargumente der atomaren Transmissionsfunktionen nicht kleiner als die untere Spaltgrenze $-S_{0} / 2$ werden, lautet

$U_{2}>-\frac{S_{0}}{2}+\frac{1}{\cos \phi^{\prime}} \max \left\{-\frac{m_{2}+m_{3}}{M} \rho_{\perp}, \frac{m_{1}}{M} \rho_{\perp}-\frac{m_{3}}{m_{2}+m_{3}} r_{\perp}, \frac{m_{1}}{M} \rho_{\perp}+\frac{m_{2}}{m_{2}+m_{3}} r_{\perp}\right\}$,

und wegen $r_{\perp}>0$ sind nur das erste und das letzte Argument der max-Funktion relevant. Mit Hilfe der Definition

$$
\Delta_{2}^{ \pm}:= \pm \frac{1}{2}\left\{-\frac{m_{2}+m_{3}-m_{1}}{M} \rho_{\perp}+\frac{m_{2}}{m_{2}+m_{3}} r_{\perp} \pm\left|\rho_{\perp}+\frac{m_{2}}{m_{2}+m_{3}} r_{\perp}\right|\right\}
$$

kann die Bedingung (3.119) geschrieben werden als

$$
U_{2}>-\frac{S_{0}}{2}+\frac{\Delta_{2}^{+}}{\cos \phi^{\prime}}
$$

Analog dazu werden die Bedingungen für die verbleibenden Quadranten aufgestellt. Für $r_{\perp}<0, U_{2}>0$ werden das erste und das letzte Argument der max-Funktion in Gleichung (3.116) benötigt. Dies führt zu

$$
U_{2}<\frac{S_{0}}{2}-\frac{\Delta_{2}^{-}}{\cos \phi^{\prime}}
$$

Im letzten Quadranten $r_{\perp}<0, U_{2}<0$ werden die ersten beiden Argumente aus Gleichung (3.119) benötigt, und es folgt

$$
U_{2}>-\frac{S_{0}}{2}+\frac{\Delta_{1}^{-}}{\cos \phi^{\prime}}
$$

\section{„Geometrischer Anteil“ und „van der Waals-Anteil“ der effektiven Spalt- breite}

Die Bedingungen für die Integrationsvariable $U_{2}$ legen nahe, das Integrationsintervall in einen Anteil mit reduzierten Integrationsgrenzen und einen Anteil, der für die verbleibenden Intervalle aufkommt, aufzuteilen. Es zeigt sich dabei, daß ersterer Anteil in Abwesenheit der attraktiven Wechselwirkung verschwindet; er wird deshalb im folgenden als „van der Waals-Anteil“ $S_{\text {eff }, \gamma}^{\mathrm{vdW}}$ der effektiven Spaltbreite bezeichnet. Der andere Anteil hingegen ist unabhängig von der attraktiven Wechselwirkung; er wird als „geometrischer Anteil" $S_{\text {eff }, \gamma}^{\text {geom }}$ bezeichnet. Die Zerlegung lautet

$$
S_{\mathrm{eff}, \gamma}\left(v^{\prime}, \overline{\theta^{\prime}}\right)=S_{\mathrm{eff}, \gamma}^{\mathrm{geom}}\left(\overline{\theta^{\prime}}\right)+S_{\mathrm{eff}, \gamma}^{\mathrm{vdW}}\left(v^{\prime}, \overline{\theta^{\prime}}\right) .
$$


Der geometrische Anteil wird definiert als

$$
\begin{aligned}
& S_{\text {eff }, \gamma}^{\text {geom }}\left(\overline{\theta^{\prime}}\right):=S_{0}-\frac{1}{\cos \phi^{\prime}} \operatorname{Re} \int_{-\infty}^{\infty} \mathrm{d} \rho_{\perp} \\
& \quad \times\left\{\int_{0}^{\infty} \mathrm{d} r_{\perp} \varphi_{\gamma \gamma}\left(\rho_{\perp}, r_{\perp}\right)\left[\Delta_{1}^{+}+\Delta_{2}^{+}\right]+\int_{-\infty}^{0} \mathrm{~d} r_{\perp} \varphi_{\gamma \gamma}\left(\rho_{\perp}, r_{\perp}\right)\left[\Delta_{1}^{-}+\Delta_{2}^{-}\right]\right\},
\end{aligned}
$$

und der van der Waals-Anteil hat die Form

$$
\begin{aligned}
& S_{\text {eff }, \gamma}^{\mathrm{vdW}}\left(v^{\prime}, \overline{\theta^{\prime}}\right):=-\operatorname{Re} \int_{-\infty}^{\infty} \mathrm{d} \rho_{\perp} \\
& \times\left\{\int_{0}^{\infty} \mathrm{d} r_{\perp} \varphi_{\gamma \gamma}\left(\rho_{\perp}, r_{\perp}\right)\left[\int_{0}^{\frac{S_{0}}{2}-\frac{\Delta_{1}^{+}}{\cos \phi^{\prime}}} \mathrm{d} U_{2} \ldots+\int_{-\frac{S_{0}}{2}+\frac{\Delta_{2}^{+}}{\cos \phi^{\prime}}}^{0} \mathrm{~d} U_{2} \ldots\right]\right. \\
& +\int_{-\infty}^{0} \mathrm{~d} r_{\perp} \varphi_{\gamma \gamma}\left(\rho_{\perp}, r_{\perp}\right)\left[\int_{0}^{\frac{S_{0}}{2}-\frac{\Delta_{2}^{-}}{\cos \phi^{\prime}}} \mathrm{d} U_{2} \ldots+\int_{-\frac{S_{0}}{2}+\frac{\Delta_{1}^{-}}{\cos \phi^{\prime}}}^{0} \mathrm{~d} U_{2}\right. \\
& \times\left[1-\tau_{1, u}^{\text {at }}\left(\frac{m_{1}}{M} \overline{\boldsymbol{P}}^{\prime} ; U_{2}+\frac{m_{2}+m_{3}}{M} \frac{\rho_{\perp}}{\cos \phi^{\prime}}\right)\right. \\
& \times \tau_{2, u}^{\text {at }}\left(\frac{m_{2}}{M} \overline{\boldsymbol{P}}^{\prime} ; U_{2}-\frac{m_{1}}{M} \frac{\rho_{\perp}}{\cos \phi^{\prime}}+\frac{m_{3}}{m_{2}+m_{3}} \frac{r_{\perp}}{\cos \phi^{\prime}}\right) \\
& \left.\left.\times \tau_{3, u}^{\text {at }}\left(\frac{m_{3}}{M} \overline{\boldsymbol{P}}^{\prime} ; U_{2}-\frac{m_{1}}{M} \frac{\rho_{\perp}}{\cos \phi^{\prime}}-\frac{m_{2}}{m_{2}+m_{3}} \frac{r_{\perp}}{\cos \phi^{\prime}}\right)\right]\right\} .
\end{aligned}
$$

(In allen vier Summanden ist derselbe Integrand einzusetzen.)

Zunächst wird der geometrische Anteil (3.125) betrachtet. Einsetzen der Definitionen von $\Delta_{1}^{ \pm}$(3.117) und $\Delta_{2}^{ \pm}$(3.120) und Zusammenfassen der beiden Integrale über $r_{\perp}$ liefert den einfachen Ausdruck

$$
\begin{aligned}
S_{\text {eff }, \gamma}^{\text {geom }}\left(\overline{\theta^{\prime}}\right) & =S_{0}-\frac{1}{2 \cos \phi^{\prime}} \operatorname{Re} \int \mathrm{d} \rho_{\perp}^{(1)} \mathrm{d} r_{\perp}^{(23)} \varphi_{\gamma \gamma}^{(1,23)}\left(\rho_{\perp}^{(1)}, r_{\perp}^{(23)}\right) \\
\times & \left(\left|r_{\perp}^{(23)}\right|+\left|\rho_{\perp}^{(1)}+\frac{m_{2}}{m_{2}+m_{3}} r_{\perp}^{(23)}\right|+\left|\rho_{\perp}^{(1)}-\frac{m_{3}}{m_{2}+m_{3}} r_{\perp}^{(23)}\right|\right) .
\end{aligned}
$$

Der Transformation der Jacobi-Koordinaten (3.4) zufolge ist der zweite Absolutbetrag in den runden Klammern aber gleich $\left|r_{\perp}^{(31)}\right|$ und der dritte gleich $\left|r_{\perp}^{(12)}\right|$. Substitution der Integrationsvariablen durch $\rho_{\perp}^{(2)}, r_{\perp}^{(31)}$ im zweiten Summanden beziehungsweise durch $\rho_{\perp}^{(3)}, r_{\perp}^{(12)}$ im dritten und Berücksichtigung der Relationen für die Dichte (3.97) liefert den folgenden Ausdruck für den geometrischen Anteil der effektiven Spaltbreite:

$$
S_{\text {eff }, \gamma}^{\text {geem }}\left(\overline{\theta^{\prime}}\right)=S_{0}-\frac{1}{2 \cos \phi^{\prime}}\left(\left\langle\left|r_{\perp}^{(23)}\right|\right\rangle_{\gamma}+\left\langle\left|r_{\perp}^{(31)}\right|\right\rangle_{\gamma}+\left\langle\left|r_{\perp}^{(12)}\right|\right\rangle_{\gamma}\right) .
$$

Die Erwartungwerte der Projektionen der Paarabstände $\mathbf{r}^{(j k)}$ senkrecht zum einlaufenden Impuls im Bindungszustand $\left|\phi_{\gamma}\right\rangle$ sind dabei definiert durch

$$
\left\langle\left|r_{\perp}^{(j k)}\right|\right\rangle_{\gamma}:=\int \mathrm{d} \rho_{\perp}^{(i)} \mathrm{d} r_{\perp}^{(j k)} \varphi_{\gamma \gamma}^{(i, j k)}\left(\rho_{\perp}^{(i)}, r_{\perp}^{(j k)}\right)\left|r_{\perp}^{(j k)}\right|
$$


In Abwesenheit der attraktiven Wechselwirkung wäre die effektive Spaltbreite der Trimerbeugung also gegenüber der geometrischen Spaltbreite $S_{0}$ reduziert um einen Term, der zum klassisch gebildeten Mittelwert der Erwartungswerte aller drei projizierten Paarabstände des Trimers proportional ist. Diese Symmetrie spiegelt die Tatsache wider, daß das Trimer keine feste räumliche Orientierung zum Transmissionsgitter besitzt. Eine physikalische Interpretation von Gleichung (3.128) wird im Unterabschnitt 3.4.4 diskutiert.

Zur Berechnung des van der Waals-Anteils der effektiven Spaltbreite wird in den vier Integralen über $U_{2}$ in der Definition (3.126) jeweils eine Substitution der Integrationsvariable durchgeführt, die die Grenzen $\pm\left[\frac{S_{0}}{2}-\frac{\Delta^{ \pm}}{\cos \phi^{\prime}}\right]$ wieder nach $\pm \frac{S_{0}}{2}$ verschiebt. Zur Verdeutlichung wird hier stellvertretend der erste der vier Summanden betrachtet. Die Substitution lautet $U_{2}^{\prime}:=U_{2}+\frac{\Delta_{1}^{+}}{\cos \phi^{\prime}}$, und der Integralausdruck wird $\mathrm{zu}$

$$
\begin{aligned}
- & \operatorname{Re} \int_{-\infty}^{\infty} \mathrm{d} \rho_{\perp} \int_{0}^{\infty} \mathrm{d} r_{\perp} \varphi_{\gamma \gamma}\left(\rho_{\perp}, r_{\perp}\right) \int_{\frac{\Delta_{1}^{+}}{\cos \phi^{\prime}}}^{\frac{S_{0}}{2}} \mathrm{~d} U_{2}^{\prime}[1- \\
& \tau_{1, u}^{\mathrm{at}}\left(\frac{m_{1}}{M} \overline{\boldsymbol{P}}^{\prime} ; U_{2}^{\prime}+\frac{1}{2 \cos \phi^{\prime}}\left[\rho_{\perp}-\frac{m_{3}}{m_{2}+m_{3}} r_{\perp}-\left|\rho_{\perp}-\frac{m_{3}}{m_{2}+m_{3}} r_{\perp}\right|\right]\right) \\
\times & \tau_{2, u}^{\mathrm{at}}\left(\frac{m_{2}}{M} \overline{\boldsymbol{P}}^{\prime} ; U_{2}^{\prime}-\frac{1}{2 \cos \phi^{\prime}}\left[\rho_{\perp}-\frac{m_{3}}{m_{2}+m_{3}} r_{\perp}+\left|\rho_{\perp}-\frac{m_{3}}{m_{2}+m_{3}} r_{\perp}\right|\right]\right) \\
\times & \left.\tau_{3, u}^{\mathrm{at}}\left(\frac{m_{3}}{M} \overline{\boldsymbol{P}}^{\prime} ; U_{2}^{\prime}-\frac{1}{2 \cos \phi^{\prime}}\left[2 r_{\perp}+\rho_{\perp}-\frac{m_{3}}{m_{2}+m_{3}} r_{\perp}+\left|\rho_{\perp}-\frac{m_{3}}{m_{2}+m_{3}} r_{\perp}\right|\right]\right)\right] .
\end{aligned}
$$

Notiert man wieder explizit die Realisierung der Jacobi-Koordinaten, so kann in den Ortsargumenten der Transmissionsfunktionen $\rho_{\perp}^{(1)}-\frac{m_{3}}{m_{2}+m_{3}} r_{\perp}^{(23)}=r_{\perp}^{(12)}$ ersetzt werden. Die Kombinationen dieser Variable lassen sich mit Hilfe der Funktion

$$
\vartheta(a):=\frac{1}{2}(a+|a|)
$$

zusammenfassen. Somit folgt

$$
\begin{aligned}
-\operatorname{Re} & \int_{-\infty}^{\infty} \mathrm{d} \rho_{\perp}^{(1)} \int_{0}^{\infty} \mathrm{d} r_{\perp}^{(23)} \varphi_{\gamma \gamma}^{(1,23)}\left(\rho_{\perp}^{(1)}, r_{\perp}^{(23)}\right) \int_{0}^{\frac{S_{0}}{2}} \mathrm{~d} U_{2}^{\prime} \\
& {\left[1-\tau_{1, u}^{\mathrm{at}}\left(\frac{m_{1}}{M} \overline{\boldsymbol{P}}^{\prime} ; U_{2}^{\prime}-\frac{1}{\cos \phi^{\prime}} \vartheta\left(-r_{\perp}^{(12)}\right)\right) \tau_{2, u}^{\mathrm{at}}\left(\frac{m_{2}}{M} \overline{\boldsymbol{P}}^{\prime} ; U_{2}^{\prime}-\frac{1}{\cos \phi^{\prime}} \vartheta\left(r_{\perp}^{(12)}\right)\right)\right.} \\
& \left.\times \tau_{3, u}^{\mathrm{at}}\left(\frac{m_{3}}{M} \overline{\boldsymbol{P}}^{\prime} ; U_{2}^{\prime}-\frac{1}{\cos \phi^{\prime}}\left[r_{\perp}^{(23)}+\vartheta\left(r_{\perp}^{(12)}\right)\right]\right)\right]
\end{aligned}
$$

wobei zur Vereinfachung die untere Integrationsgrenze $\Delta_{1}^{+} / \cos \phi^{\prime}$ des Integrals über $U_{2}^{\prime}$ durch Null ersetzt wurde. Dies ist möglich, da das Intervall $\left[0, \Delta_{1}^{+} / \cos \phi^{\prime}\right]$ wegen der räumlichen Lokalisierung der Dichte einen kleinen Bereich links oder rechts der Spaltmitte darstellt, innerhalb dessen der Integrand des Ausdrucks (3.132) vernachlässigbar klein ist. 
Die anderen drei Integralterme in der Definition des van der Waals-Anteils der effektiven Spaltbreite (3.126) können entsprechend umgeformt werden. Für den allgemeinen Fall eines aus unterschiedlichen Teilchen bestehenden Trimers lautet der endgültige Ausdruck für den van der Waals-Anteil der effektiven Spaltbreite schließlich

$$
\begin{aligned}
& S_{\text {eff }, \gamma}^{\mathrm{vdW}}\left(v^{\prime}, \overline{\theta^{\prime}}\right) \simeq-\operatorname{Re} \int_{-\infty}^{\infty} \mathrm{d} \rho_{\perp}^{(1)}\{ \\
& \int_{0}^{\infty} \mathrm{d} r_{\perp}^{(23)} \varphi_{\gamma \gamma}^{(1,23)}\left(\rho_{\perp}^{(1)}, r_{\perp}^{(23)}\right) \int_{0}^{\frac{S_{0}}{2}} \mathrm{~d} U_{2}^{\prime} \\
& {\left[1-\tau_{1, u}^{\mathrm{at}}\left(\frac{m_{1}}{M} \overline{\boldsymbol{P}}^{\prime} ; U_{2}^{\prime}-\frac{1}{\cos \phi^{\prime}} \vartheta\left(-r_{\perp}^{(12)}\right)\right) \tau_{2, u}^{\mathrm{at}}\left(\frac{m_{2}}{M} \overline{\boldsymbol{P}}^{\prime} ; U_{2}^{\prime}-\frac{1}{\cos \phi^{\prime}} \vartheta\left(r_{\perp}^{(12)}\right)\right)\right.} \\
& \left.\times \tau_{3, u}^{\mathrm{at}}\left(\frac{m_{3}}{M} \overline{\boldsymbol{P}}^{\prime} ; U_{2}^{\prime}-\frac{1}{\cos \phi^{\prime}}\left[r_{\perp}^{(23)}+\vartheta\left(r_{\perp}^{(12)}\right)\right]\right)\right] \\
& +\int_{0}^{\infty} \mathrm{d} r_{\perp}^{(23)} \varphi_{\gamma \gamma}^{(1,23)}\left(\rho_{\perp}^{(1)}, r_{\perp}^{(23)}\right) \int_{-\frac{S_{0}}{2}}^{0} \mathrm{~d} U_{2}^{\prime} \\
& {\left[1-\tau_{1, u}^{\mathrm{at}}\left(\frac{m_{1}}{M} \overline{\boldsymbol{P}}^{\prime} ; U_{2}^{\prime}+\frac{1}{\cos \phi^{\prime}} \vartheta\left(-r_{\perp}^{(31)}\right)\right) \tau_{3, u}^{\mathrm{at}}\left(\frac{m_{3}}{M} \overline{\boldsymbol{P}}^{\prime} ; U_{2}^{\prime}+\frac{1}{\cos \phi^{\prime}} \vartheta\left(r_{\perp}^{(31)}\right)\right)\right.} \\
& \left.\times \tau_{2, u}^{\mathrm{at}}\left(\frac{m_{2}}{M} \overline{\boldsymbol{P}}^{\prime} ; U_{2}^{\prime}+\frac{1}{\cos \phi^{\prime}}\left[r_{\perp}^{(23)}+\vartheta\left(r_{\perp}^{(31)}\right)\right]\right)\right] \\
& +\int_{-\infty}^{0} \mathrm{~d} r_{\perp}^{(23)} \varphi_{\gamma \gamma}^{(1,23)}\left(\rho_{\perp}^{(1)}, r_{\perp}^{(23)}\right) \int_{0}^{\frac{S_{0}}{2}} \mathrm{~d} U_{2}^{\prime} \\
& {\left[1-\tau_{3, u}^{\mathrm{at}}\left(\frac{m_{3}}{M} \overline{\boldsymbol{P}}^{\prime} ; U_{2}^{\prime}-\frac{1}{\cos \phi^{\prime}} \vartheta\left(-r_{\perp}^{(31)}\right)\right) \tau_{1, u}^{\mathrm{at}}\left(\frac{m_{1}}{M} \overline{\boldsymbol{P}}^{\prime} ; U_{2}^{\prime}-\frac{1}{\cos \phi^{\prime}} \vartheta\left(r_{\perp}^{(31)}\right)\right)\right.} \\
& \left.\times \tau_{2, u}^{\mathrm{at}}\left(\frac{m_{2}}{M} \overline{\boldsymbol{P}}^{\prime} ; U_{2}^{\prime}+\frac{1}{\cos \phi^{\prime}}\left[r_{\perp}^{(23)}-\vartheta\left(-r_{\perp}^{(31)}\right)\right]\right)\right] \\
& +\int_{-\infty}^{0} \mathrm{~d} r_{\perp}^{(23)} \varphi_{\gamma \gamma}^{(1,23)}\left(\rho_{\perp}^{(1)}, r_{\perp}^{(23)}\right) \int_{-\frac{S_{0}}{2}}^{0} \mathrm{~d} U_{2}^{\prime} \\
& {\left[1-\tau_{2, u}^{\text {at }}\left(\frac{m_{2}}{M} \overline{\boldsymbol{P}}^{\prime} ; U_{2}^{\prime}+\frac{1}{\cos \phi^{\prime}} \vartheta\left(-r_{\perp}^{(12)}\right)\right) \tau_{1, u}^{\text {at }}\left(\frac{m_{1}}{M} \overline{\boldsymbol{P}}^{\prime} ; U_{2}^{\prime}+\frac{1}{\cos \phi^{\prime}} \vartheta\left(r_{\perp}^{(12)}\right)\right)\right.} \\
& \left.\left.\times \tau_{3, u}^{\text {at }}\left(\frac{m_{3}}{M} \overline{\boldsymbol{P}}^{\prime} ; U_{2}^{\prime}-\frac{1}{\cos \phi^{\prime}}\left[r_{\perp}^{(23)}-\vartheta\left(-r_{\perp}^{(12)}\right)\right]\right)\right]\right\} .
\end{aligned}
$$

\section{Die effektive Spaltbreite spinloser, ununterscheidbarer Teilchen}

Weitere Vereinfachungen lassen sich aus Symmetriegründen im Falle spinloser, ununterscheidbarer Teilchen erzielen. Einige Eigenschaften der Bindungswellenfunktionen solcher Trimere wurden bereits in Kapitel 3.1.2 untersucht. Wegen der Ununterscheidbarkeit ist die Kennzeichnung der Realisierungen der Jacobi-Koordinaten durch Teilchenindizes nicht zweckmäßig. Stattdessen werden die Relativkoordinaten der drei Realisierungen nun mit $\left(\begin{array}{c}\rho \\ \mathbf{r}\end{array}\right),\left(\begin{array}{c}\rho^{\prime} \\ \mathbf{r}^{\prime}\end{array}\right):=\mathbb{d}\left(\begin{array}{l}\rho \\ \mathbf{r}\end{array}\right)$ und $\left(\begin{array}{l}\rho^{\prime \prime} \\ \mathbf{r}^{\prime \prime}\end{array}\right):=\mathbb{d}^{2}\left(\begin{array}{l}\rho \\ \mathbf{r}\end{array}\right)$ bezeichnet. 
Die vollständig symmetrisierten Trimer-Bindungswellenfunktionen erfüllen aufgrund ihrer Konstruktion (3.31) die Beziehungen $\phi_{\gamma}(\boldsymbol{\rho}, \mathbf{r})=\phi_{\gamma}\left(\boldsymbol{\rho}^{\prime}, \mathbf{r}^{\prime}\right)=\phi_{\gamma}\left(\boldsymbol{\rho}^{\prime \prime}, \mathbf{r}^{\prime \prime}\right)$. Diese Eigenschaft überträgt sich unmittelbar auf die Dichte $\varphi_{\gamma \gamma}\left(\rho_{\perp}, r_{\perp}\right)$.

Als erste Konsequenz sind die im geometrischen Anteil der effektiven Spaltbreite (3.128) auftretenden Erwartungswerte der drei projizierten Paarabstände $r_{\perp}, r_{\perp}^{\prime}$ und $r_{\perp}^{\prime \prime}$ identisch. Es folgt also

$$
S_{\mathrm{eff}, \gamma}^{\text {geom }}\left(\overline{\theta^{\prime}}\right)=S_{0}-\frac{3}{2} \frac{\left\langle\left|r_{\perp}\right|\right\rangle_{\gamma}}{\cos \phi^{\prime}} .
$$

In Anhang B wird eine spezielle Approximation für Trimerzustände zum Gesamtdrehimpuls $L=0$ abgeleitet: wird die Zweiteilchen-Streumatrix der drei Teilchenpaare in der Nähe der Trimer-Bindungsenergie durch den Pol eines gebundenen Dimerzustandes zur Drehimpulsquantenzahl $l=0$ dominiert, so gilt für das Trimer $\left\langle\left|r_{\perp}\right|\right\rangle_{\gamma} \simeq \frac{1}{2}\langle r\rangle_{\gamma}$ mit $r:=|\mathbf{r}|$. Da speziell das Helium-Helium-Paarpotential nur einen, sehr schwach gebundenen Dimer-Zustand trägt [5], sollte diese Näherung im HeliumTrimer besonders gut anwendbar sein. So erhält man aus Gleichung (3.134)

$$
S_{\text {eff }, \gamma}^{\text {geom }}\left(\overline{\theta^{\prime}}\right) \simeq S_{0}-\frac{3}{4} \frac{\langle r\rangle_{\gamma}}{\cos \phi^{\prime}} .
$$

Auch der van der Waals-Anteil der effektiven Spaltbreite (3.133) erlaubt weitere Vereinfachungen. Beispielhaft wird wieder der erste der vier Integralterme aus Gleichung (3.133) betrachtet. Da die atomaren Transmissionsfunktionen nun unabhängig vom Teilchenindex sind, kann ihr Produkt folgendermaßen umgeformt werden:

$$
\begin{aligned}
\tau_{u}^{\text {at }}\left(\frac{1}{3} \overline{\boldsymbol{P}}^{\prime} ; U_{2}^{\prime}\right. & \left.-\frac{1}{\cos \phi^{\prime}} \vartheta\left(-r_{\perp}^{\prime \prime}\right)\right) \tau_{u}^{\mathrm{at}}\left(\frac{1}{3} \overline{\boldsymbol{P}}^{\prime} ; U_{2}^{\prime}-\frac{1}{\cos \phi^{\prime}} \vartheta\left(r_{\perp}^{\prime \prime}\right)\right) \\
& =\tau_{u}^{\mathrm{at}}\left(\frac{1}{3} \overline{\boldsymbol{P}}^{\prime} ; U_{2}^{\prime}-\frac{\left|r_{\perp}^{\prime \prime}\right| \Theta\left(-r_{\perp}^{\prime \prime}\right)}{\cos \phi^{\prime}}\right) \tau_{u}^{\mathrm{at}}\left(\frac{1}{3} \overline{\boldsymbol{P}}^{\prime} ; U_{2}^{\prime}-\frac{\left|r_{\perp}^{\prime \prime}\right| \Theta\left(r_{\perp}^{\prime \prime}\right)}{\cos \phi^{\prime}}\right) \\
& =\tau_{u}^{\mathrm{at}}\left(\frac{1}{3} \overline{\boldsymbol{P}}^{\prime} ; U_{2}^{\prime}\right) \tau_{u}^{\mathrm{at}}\left(\frac{1}{3} \overline{\boldsymbol{P}}^{\prime} ; U_{2}^{\prime}-\frac{\left|r_{\perp}^{\prime \prime}\right|}{\cos \phi^{\prime}}\right) .
\end{aligned}
$$

Hierzu wurde $\vartheta(a)=a \Theta(a)$ ausgenutzt, wobei $\Theta(a)$ die Heaviside-Funktion bezeichnet. Außerdem wurde $m=M / 3$ eingesetzt. Entsprechend können die anderen Paare von Transmissionsfunktionen in Gleichung (3.133) vereinfacht werden. Anschließend ist es möglich, die Integrationen über $r_{\perp}$ wieder zusammenzufassen. Mit Hilfe der Beziehungen

$$
r_{\perp}^{\prime}=-\rho_{\perp}-\frac{1}{2} r_{\perp} \quad \text { und } \quad r_{\perp}^{\prime \prime}=\rho_{\perp}-\frac{1}{2} r_{\perp}
$$

folgt der van der Waals-Anteil der effektiven Spaltbreite

$$
\begin{aligned}
& S_{\text {eff, }, \gamma}^{\mathrm{ddW}}\left(v^{\prime}, \overline{\theta^{\prime}}\right) \simeq-\operatorname{Re} \int_{-\infty}^{\infty} \mathrm{d} \rho_{\perp} \int_{-\infty}^{\infty} \mathrm{d} r_{\perp} \varphi_{\gamma \gamma}\left(\rho_{\perp}, r_{\perp}\right) \\
& \left\{\int _ { 0 } ^ { \frac { S _ { 0 } } { 2 } } \mathrm { d } U _ { 2 } ^ { \prime } \left[1-\tau_{u}^{\mathrm{at}}\left(\frac{1}{3} \overline{\boldsymbol{P}}^{\prime} ; U_{2}^{\prime}\right) \tau_{u}^{\mathrm{at}}\left(\frac{1}{3} \overline{\boldsymbol{P}}^{\prime} ; U_{2}^{\prime}-\frac{\left|\rho_{\perp}-\frac{1}{2}\right| r_{\perp}||}{\cos \phi^{\prime}}\right)\right.\right.
\end{aligned}
$$




$$
\begin{gathered}
\left.\times \tau_{u}^{\mathrm{at}}\left(\frac{1}{3} \overline{\boldsymbol{P}}^{\prime} ; U_{2}^{\prime}-\frac{\left|r_{\perp}\right|+\left(\rho_{\perp}-\frac{1}{2}\left|r_{\perp}\right|\right) \Theta\left(\rho_{\perp}-\frac{1}{2}\left|r_{\perp}\right|\right)}{\cos \phi^{\prime}}\right)\right] \\
+\int_{-\frac{S_{0}}{2}}^{0} \mathrm{~d} U_{2}^{\prime}\left[1-\tau_{u}^{\mathrm{at}}\left(\frac{1}{3} \overline{\boldsymbol{P}}^{\prime} ; U_{2}^{\prime}\right) \tau_{u}^{\mathrm{at}}\left(\frac{1}{3} \overline{\boldsymbol{P}}^{\prime} ; U_{2}^{\prime}+\frac{\left|\rho_{\perp}+\frac{1}{2}\right| r_{\perp}||}{\cos \phi^{\prime}}\right)\right. \\
\left.\left.\times \tau_{u}^{\mathrm{at}}\left(\frac{1}{3} \overline{\boldsymbol{P}}^{\prime} ; U_{2}^{\prime}+\frac{\left|r_{\perp}\right|+\left(-\rho_{\perp}-\frac{1}{2}\left|r_{\perp}\right|\right) \Theta\left(-\rho_{\perp}-\frac{1}{2}\left|r_{\perp}\right|\right)}{\cos \phi^{\prime}}\right)\right]\right\} .
\end{gathered}
$$

Der van der Waals-Anteil der effektiven Spaltbreite entspricht also dem negativen Realteil des Erwartungswerts des Ausdrucks in geschweiften Klammern im TrimerBindungszustand $\left|\phi_{\gamma}\right\rangle$. Die Interpretation der Ortsargumente der Transmissionsfunktionen als Teilchenpositionen ist leider nicht offensichtlich. Sind die Funktionen in den geschweiften Klammern aber langsam veränderlich auf der räumlichen Ausdehnung des Bindungszustandes, so sollte es näherungsweise möglich sein, die Bildung des Erwartungswerts mit der Auswertung der Funktionen zu vertauschen. Erst dadurch würde auch die Evaluation experimenteller Trimer-Beugungsbilder bei endlicher Strahlgeschwindigkeit ermöglicht, denn auf diese Weise wäre der van der WaalsAnteil der effektiven Spaltbreite nur noch von den Erwartungswerten der Funktionsargumente abhängig, nicht aber vom gesamten, komplizierten Bindungszustand. Aus der Untersuchung des Helium-Dimers ist bekannt, daß eine solche zunächst grob erscheinende Approximation durchaus möglich ist [5]. Ihre quantitative Genauigkeit hängt von der Form des Bindungszustandes ab und sollte im Einzelfall mit einem numerischen Modell geprüft werden. Dies wird unten für das Helium-Trimer durchgeführt.

Zunächst wird die Anwendbarkeit dieser Näherung vorausgesetzt. Es müssen also die Erwartungswerte der Kombinationen von Relativkoordinaten in den Ortsargumenten der Transmissionsfunktionen in Gleichung (3.136) berechnet werden. Für die Kombination $\left|\rho_{\perp}-\frac{1}{2}\right| r_{\perp}||$ gilt

$$
\begin{aligned}
\langle| \rho_{\perp} & -\frac{1}{2}\left|r_{\perp}\right||\rangle_{\gamma}=\int \mathrm{d} \rho_{\perp} \mathrm{d} r_{\perp} \varphi_{\gamma \gamma}\left(\rho_{\perp}, r_{\perp}\right)\left|\rho_{\perp}-\frac{1}{2}\right| r_{\perp}|| \\
& =\int \mathrm{d} \rho_{\perp} \mathrm{d} r_{\perp} \varphi_{\gamma \gamma}\left(\rho_{\perp}, r_{\perp}\right)\left\{\left|\rho_{\perp}-\frac{1}{2} r_{\perp}\right| \Theta\left(r_{\perp}\right)+\left|\rho_{\perp}+\frac{1}{2} r_{\perp}\right| \Theta\left(-r_{\perp}\right)\right\} .
\end{aligned}
$$

Wegen der festen Parität der symmetrisierten Bindungswellenfunktionen unter Inversion von $\boldsymbol{\rho}$ (vgl. Gl. 3.33) ist $\varphi_{\gamma \gamma}\left(\rho_{\perp}, r_{\perp}\right)$ gerade in $\rho_{\perp}$. Somit kann innerhalb des zweiten Absolutbetrags das Vorzeichen von $\rho_{\perp}$ invertiert werden, und es folgt, daß der gesamte Ausdruck gleich dem Erwartungswert der Größe $\left|\rho_{\perp}-\frac{1}{2} r_{\perp}\right| \equiv\left|r_{\perp}^{\prime \prime}\right|$ ist. Wegen der Gleichheit aller Paarabstände gilt daher

$$
\left\langle\left|\rho_{\perp}-\frac{1}{2}\right| r_{\perp}||\right\rangle_{\gamma}=\left\langle\left|r_{\perp}^{\prime \prime}\right|\right\rangle_{\gamma}=\left\langle\left|r_{\perp}\right|\right\rangle_{\gamma}
$$

Entsprechend folgt $\left\langle\left|\rho_{\perp}+\frac{1}{2}\right| r_{\perp}||\right\rangle_{\gamma}=\left\langle\left|r_{\perp}\right|\right\rangle_{\gamma}$. Die in der dritten Transmissionfunktion auftretende Kombination $\left(\rho_{\perp}-\frac{1}{2}\left|r_{\perp}\right|\right) \Theta\left(\rho_{\perp}-\frac{1}{2}\left|r_{\perp}\right|\right)$ ist zunächst mittels Glei- 
chung (3.131) wieder zu zerlegen. Man findet unter Verwendung des zuvor berechneten Erwartungswerts (3.137)

$$
\begin{aligned}
\left\langle\left(\rho_{\perp}-\frac{1}{2}\left|r_{\perp}\right|\right) \Theta\left(\rho_{\perp}-\frac{1}{2}\left|r_{\perp}\right|\right)\right\rangle_{\gamma} & =\left\langle\vartheta\left(\rho_{\perp}-\frac{1}{2}\left|r_{\perp}\right|\right)\right\rangle_{\gamma} \\
& =\frac{1}{2}\left\langle\rho_{\perp}-\frac{1}{2}\left|r_{\perp}\right|+\left|\rho_{\perp}-\frac{1}{2}\right| r_{\perp}||\right\rangle_{\gamma} \\
& =\frac{1}{4}\left\langle\left|r_{\perp}\right|\right\rangle_{\gamma},
\end{aligned}
$$

wobei verwendet wurde, daß aus der geraden Parität der Dichte auch der Erwartungswert $\left\langle\rho_{\perp}\right\rangle_{\gamma}=0$ folgt. Entsprechend wird die noch verbleibende Kombination $\left(-\rho_{\perp}-\frac{1}{2}\left|r_{\perp}\right|\right) \Theta\left(-\rho_{\perp}-\frac{1}{2}\left|r_{\perp}\right|\right)$ ausgewertet. Durch Einsetzen in die Gleichung (3.136) erhält man so die approximative, nur vom projizierten Paarabstand $\left\langle\left|r_{\perp}\right|\right\rangle_{\gamma}$ abhängige Formulierung des van der Waals-Anteils der effektiven Spaltbreite

$$
\begin{aligned}
S_{\mathrm{eff}, \gamma}^{\mathrm{vdW}}\left(v^{\prime}, \overline{\theta^{\prime}}\right) \simeq-\operatorname{Re}\left\{\int_{0}^{\frac{S_{0}}{2}} \mathrm{~d} U_{2}^{\prime}[1\right. & -\tau_{u}^{\mathrm{at}}\left(\frac{1}{3} \overline{\boldsymbol{P}}^{\prime} ; U_{2}^{\prime}\right) \tau_{u}^{\mathrm{at}}\left(\frac{1}{3} \overline{\boldsymbol{P}}^{\prime} ; U_{2}^{\prime}-\frac{\left\langle\left|r_{\perp}\right|\right\rangle_{\gamma}}{\cos \phi^{\prime}}\right) \\
& \left.\times \tau_{u}^{\mathrm{at}}\left(\frac{1}{3} \overline{\boldsymbol{P}}^{\prime} ; U_{2}^{\prime}-\frac{5}{4} \frac{\left\langle r_{\perp} \mid\right\rangle_{\gamma}}{\cos \phi^{\prime}}\right)\right] \\
+\int_{-\frac{S_{0}}{2}}^{0} \mathrm{~d} U_{2}^{\prime}[1 & -\tau_{u}^{\mathrm{at}}\left(\frac{1}{3} \overline{\boldsymbol{P}}^{\prime} ; U_{2}^{\prime}\right) \tau_{u}^{\mathrm{at}}\left(\frac{1}{3} \overline{\boldsymbol{P}}^{\prime} ; U_{2}^{\prime}+\frac{\left\langle\left|r_{\perp}\right|\right\rangle_{\gamma}}{\cos \phi^{\prime}}\right) \\
& \left.\left.\times \tau_{u}^{\mathrm{at}}\left(\frac{1}{3} \overline{\boldsymbol{P}}^{\prime} ; U_{2}^{\prime}+\frac{5}{4} \frac{\left\langle\left|r_{\perp}\right|\right\rangle_{\gamma}}{\cos \phi^{\prime}}\right)\right]\right\} .
\end{aligned}
$$

Unter der Voraussetzung, die bereits von Gleichung (3.134) zu (3.135) führte, kann schließlich der projizierte Paarabstand näherungsweise wieder durch $\frac{1}{2}\langle r\rangle_{\gamma}$ ersetzt werden.

Im Hinblick auf die Auswertung der Experimente von Brühl, Kalinin, Kornilov und Toennies wird nun die Approximation des van der Waals-Anteils (3.136) durch den Ausdruck (3.138) unter Verwendung von $\left\langle\left|r_{\perp}\right|\right\rangle_{\gamma} \simeq \frac{1}{2}\langle r\rangle_{\gamma}$ für die beiden Bindungszustände des Helium-Trimers quantitativ untersucht. Wegen der geringeren Streuung $\sqrt{\left\langle r^{2}\right\rangle_{\gamma}}$ um den Erwartungswert $\langle r\rangle_{\gamma}$ ist zu erwarten, daß die Näherung für den Grundzustand von ${ }^{4} \mathrm{He}_{3}$ günstiger ausfällt als für den angeregten Zustand, dessen Streuung um den Faktor zehn größer ist (vgl. Tab. 3.1). In Abbildung 3.5 wird die (gesamte) effektive Spaltbreite mit Hilfe der theoretisch berechneten Bindungswellenfunktionen des Helium-Trimers (vgl. Anh. B) vor und nach der Näherung verglichen. Der Approximationsfehler $\delta_{\gamma}:=S_{\text {eff, } \gamma}^{\mathrm{vdW}}(3.136)-S_{\text {eff, }, \gamma}^{\mathrm{vdW}}(3.138)$ beträgt bei den für Abbildung 3.5 gewählten, experimentell relevanten Parametern im Grundzustand im Mittel $\delta_{\gamma}=-2,2 \AA$ und im angeregten Zustand $\delta_{\gamma}=-7,2 \AA$. Dieser Fehler verursacht bei der Anpassung der Formel (3.138) an experimentelle Werte der effektiven Spaltbreite mittels des Parameters $\langle r\rangle_{\gamma}$ eine systematische Überschätzung von $\langle r\rangle_{\gamma}$ um den Wert $\frac{4}{3} \delta_{\gamma} \cos \phi^{\prime} \approx 0,3 \delta_{\gamma}$ entsprechend $7 \%$ im Grundzustand und $3 \% \mathrm{im}$ 


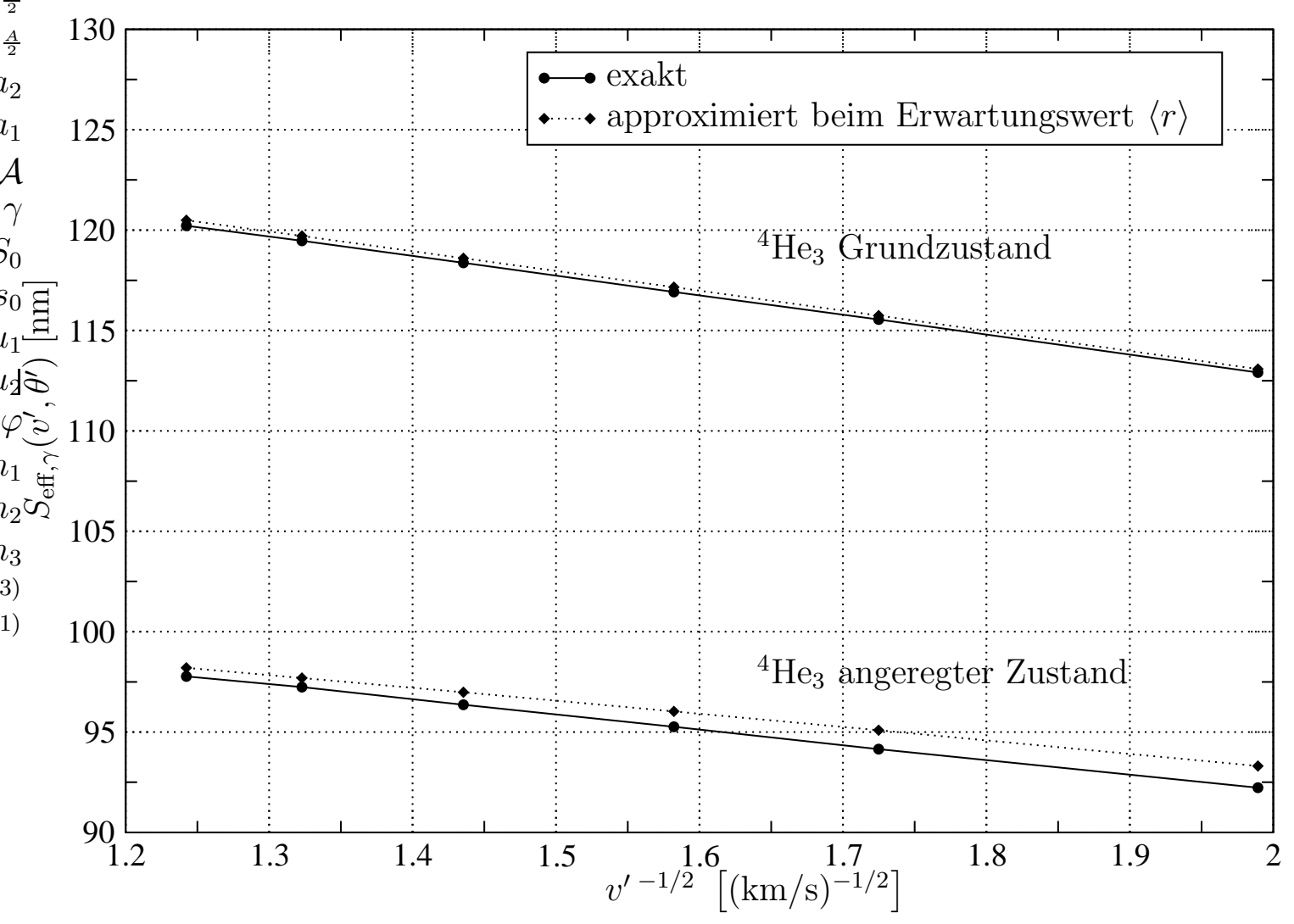

Abbildung 3.5: Vergleich der effektiven Spaltbreite $S_{\text {eff, } \gamma}\left(v^{\prime}, \overline{\theta^{\prime}}\right)$ vor und nach der Approximation des van der Waals-Anteils $S_{\mathrm{eff}, \gamma}^{\mathrm{vdW}}\left(v^{\prime}, \overline{\theta^{\prime}}\right)$ für den Grundzustand und den angeregten Zustand des Helium-Trimers ${ }^{4} \mathrm{He}_{3}$. Die durchgezogenen Kurven zeigen jeweils die (gesamte) effektive Spaltbreite, deren van der Waals-Anteil nach Gleichung (3.136) unter Verwendung theoretischer Bindungswellenfunktionen (vgl. Anh. B) berechnet wurde; für die gepunkteten Kurven wurde der van der Waals-Anteil nach der approximierten Gleichung (3.138) berechnet. Erwartungsgemäß fällt die Approximation für den kompakten Grundzustand deutlich genauer aus als für den räumlich stärker ausgedehnten angeregten Zustand. Die Auftragung gegen $v^{\prime-1 / 2}$ soll nicht den Eindruck erwecken, daß für die effektive Spaltbreite des Helium-Trimers eine asymptotische Formel analog zu (2.77) gälte. Tatsächlich erscheinen die Kurven nur im dargestellten, experimentell relevanten Geschwindigkeitsintervall nahezu linear, nicht aber für $v^{\prime} \rightarrow \infty$. 
angeregten Zustand. Dies ist bei der Interpretation der Ergebnisse von Experimenten zu beachten.

\subsubsection{Trimerbeugung am Gitter bei senkrechtem Einfall}

Einige frühe Experimente zur Trimerbeugung wurden bei senkrechtem Einfall durchgeführt. Die Ableitung der zugehörigen Formeln für die Trimer-Übergangsamplitude und die Intensität im Beugungsbild erfolgt prinzipiell analog zum Vorgehen bei nichtsenkrechtem Einfall. Sie können auch formal aus den Ergebnissen des letzten Kapitels übernommen werden, wenn die Winkel zu $\varphi=\pi / 2$ und $\theta^{\prime}=0$, und somit $\phi^{\prime}=0$, gewählt werden. Dadurch gehen auch die Impulskomponenten $\Pi_{k}^{\prime}$ in $P_{k}^{\prime}$ und $\Pi_{k}$ in $P_{k}(k=1,2)$ über. Man findet, daß die Trimer-Spaltamplitude durch den Ausdruck

$$
a_{x, \gamma \gamma^{\prime}}^{\mathrm{tri}}\left(\boldsymbol{P}^{\prime} ; \Delta P_{2}\right):=\int_{-\frac{s_{0}}{2}}^{\frac{s_{0}}{2}} \mathrm{~d} X_{2} \exp \left(-\mathrm{i} \Delta P_{2} X_{2} / \hbar\right) \tau_{x, \gamma \gamma^{\prime}}^{\mathrm{tri}}\left(\boldsymbol{P}^{\prime} ; X_{2}\right)
$$

gegeben ist, wobei die zugehörige Trimer-Transmissionsfunktion durch

$$
\begin{aligned}
\tau_{x, \gamma \gamma^{\prime}}^{\mathrm{tri}}\left(\boldsymbol{P}^{\prime} ; X_{2}\right) & :=\int \mathrm{d} \rho_{2}^{(1)} \mathrm{d} r_{2}^{(23)} \varphi_{\gamma \gamma^{\prime}}^{(1,23)}\left(\rho_{2}^{(1)}, r_{2}^{(23)}\right) \\
& \times \tau_{1, x}^{\text {at }}\left(\frac{m_{1}}{M} \boldsymbol{P}^{\prime} ; r_{12}\right) \tau_{2, x}^{\text {at }}\left(\frac{m_{2}}{M} \boldsymbol{P}^{\prime} ; r_{22}\right) \tau_{3, x}^{\text {at }}\left(\frac{m_{3}}{M} \boldsymbol{P}^{\prime} ; r_{32}\right)
\end{aligned}
$$

definiert ist. In diesem Fall kann die Variable $X_{2}$ als Projektion des Trimer-Schwerpunkts auf die $x_{2}$-Achse interpretiert werden. Die Projektionen der Teilchenorte lauten

$$
\begin{aligned}
& r_{12}=X_{2}+\frac{m_{2}+m_{3}}{M} \rho_{2}^{(1)} \\
& r_{22}=X_{2}-\frac{m_{1}}{M} \rho_{2}^{(1)}+\frac{m_{3}}{m_{2}+m_{3}} r_{2}^{(23)} \\
& r_{32}=X_{2}-\frac{m_{1}}{M} \rho_{2}^{(1)}-\frac{m_{2}}{m_{2}+m_{3}} r_{2}^{(23)} .
\end{aligned}
$$

Die atomare Transmissionsfunktion für senkrechten Einfall $\tau_{x}^{\text {at }}\left(\boldsymbol{p}^{\prime} ; x_{2}\right)$ wird in Abschnitt A.1.2 angegeben.

Aufgrund der Spiegelsymmetrie des Transmissionsgitters bezüglich der Koordinatenrichtung $x_{2}$ vereinfacht sich die Formel für die Intensität der Beugungsordnungen elastischer Beugung (3.113) bei senkrechtem Einfall. Die Kumulanten erfüllen $R_{j, \gamma}^{+}=R_{j, \gamma}^{-}$, und somit gilt $\alpha_{\gamma}=0, \Omega_{R, \gamma}=0$ und $\Omega_{I, \gamma}=0$. Außerdem geht der Wellenzahlübertrag (3.112) über in $K\left(\theta_{n}\right)=n 2 \pi / d$. Um den Unterschied zum nichtsenkrechten Einfall anzudeuten, werden die verbleibenden Funktionen $\Delta_{\gamma}(3.108)$ und $\Sigma_{\gamma}$ (3.110) mit kleinen griechischen Buchstaben bezeichnet. Es gilt also für die elastische Trimerbeugung

$$
I_{n}^{\gamma \gamma} \propto \mathrm{e}^{-\left(n 2 \pi \sigma_{\gamma} / d\right)^{2}} \frac{\sin ^{2}\left[n \pi s_{\text {eff }, \gamma} / d\right]+\sinh ^{2}\left[n \pi \delta_{\gamma} / d\right]}{[n \pi / d]^{2}},
$$


wobei der winkelabhängige Vorfaktor $\left[\cos \theta_{n}+1\right]^{2}$ vernachlässigt wurde. Diese Formel ist bereits von der Atombeugung (Gl. (2.80) und Ref. [18]) und der Dimerbeugung [5] bekannt.

Für den geometrischen Anteil der effektiven Spaltbreite $s_{\mathrm{eff}, \gamma}\left(v^{\prime}\right)$ erhält man den einfacheren Ausdruck

$$
s_{\mathrm{eff}, \gamma}^{\text {geom }}=s_{0}-\frac{1}{2}\left(\left\langle\left|r_{2}^{(23)}\right|\right\rangle_{\gamma}+\left\langle\left|r_{2}^{(31)}\right|\right\rangle_{\gamma}+\left\langle\left|r_{2}^{(12)}\right|\right\rangle_{\gamma}\right),
$$

und der van der Waals-Anteil geht analog zu der Trimer-Transmissionsfunktion (3.140) aus Gleichung (3.133) hervor. Entsprechend erhält man die speziellen Formeln für spinlose, ununterscheidbare Teilchen.

\subsubsection{Physikalische Interpretation der effektiven Spaltbreite}

Aus früheren Arbeiten über die Beugung von Dimeren ist bekannt, daß der geometrische Anteil der effektiven Spaltbreite des Dimers bei senkrechtem Einfall durch $s_{\text {eff }, \gamma}^{\text {geom }}=s_{0}-\left\langle\left|r_{2}\right|\right\rangle$ gegeben ist [5], wobei $\mathbf{r}$ die Relativkoordinate des Dimers bezeichnet. Der Term $\left\langle\left|r_{2}\right|\right\rangle$ kann interpretiert werden als die „Breite“ des Dimers senkrecht zur Einfallsrichtung (vgl. Abb. 3.6a). Die Ergebnisse dieses Kapitels für die effektive Spaltbreite des Trimers erlauben eine ähnlich anschauliche Interpretation. Zunächst wird der senkrechte Einfall betrachtet. Wie an Abbildung $3.6 \mathrm{~b}$ abzulesen ist, gibt
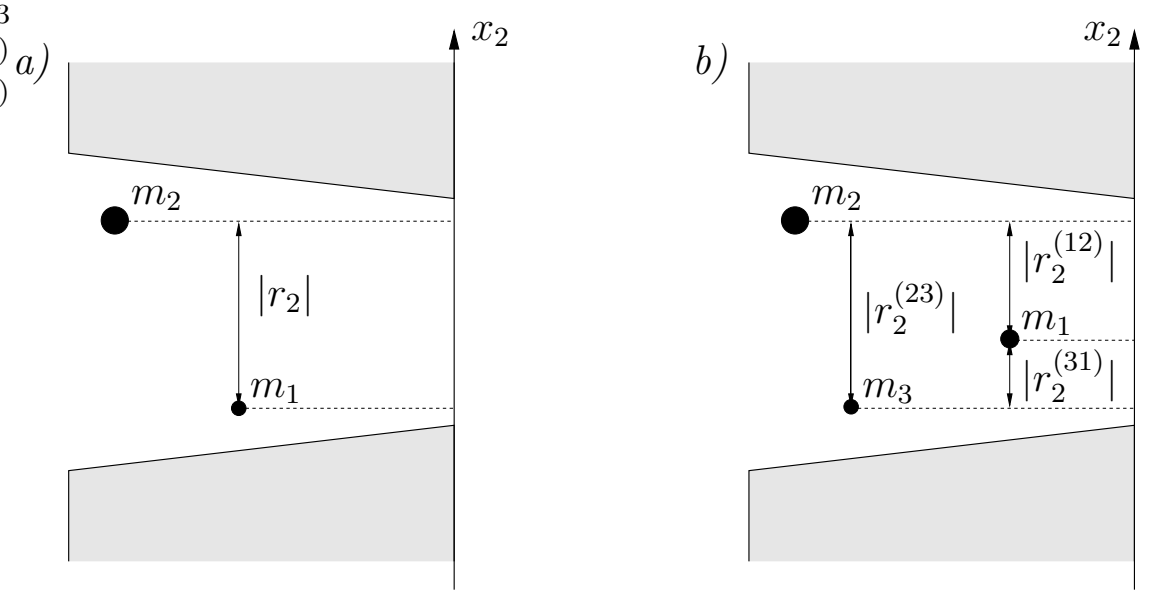

Abbildung 3.6: Interpretation des geometrischen Anteils der effektiven Spaltbreite bei senkrechtem Einfall. a) Die „Breite“ des Dimers im Spalt ist $\left\langle\left|r_{2}\right|\right\rangle$. b) Die „Breite“ des Trimers ist gegeben durch die symmetrische Summe $\frac{1}{2}\left(\left\langle\left|r_{2}^{(23)}\right|\right\rangle+\left\langle\left|r_{2}^{(31)}\right|\right\rangle+\left\langle\left|r_{2}^{(12)}\right|\right\rangle\right)$.

die symmetrische Summe

$$
\frac{1}{2}\left(\left\langle\left|r_{2}^{(23)}\right|\right\rangle+\left\langle\left|r_{2}^{(31)}\right|\right\rangle+\left\langle\left|r_{2}^{(12)}\right|\right\rangle\right)
$$

unabhängig von der relativen Lage der drei Massen die „Breite“ des Trimers senkrecht zur Einfallsrichtung an. Es ist daher anschaulich verständlich, daß im geometrischen 


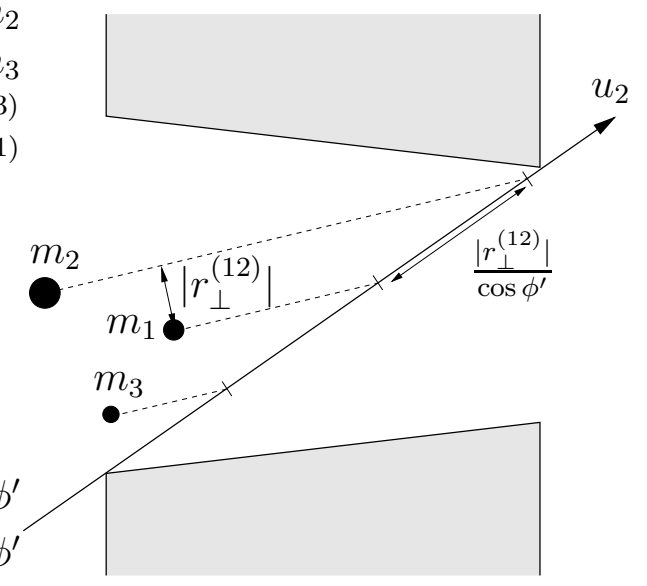

Abbildung 3.7: Interpretation des geometrischen Anteils der effektiven Spaltbreite bei nicht-senkrechtem Einfall. Die Größe $\left|r_{\perp}^{(12)}\right| / \cos \phi^{\prime}$ ist die senkrechte Projektion des Paarabstandes $\left|r_{\perp}^{(12)}\right|$ auf die $u_{2}$-Koordinatenachse. Entsprechendes gilt für die anderen Paarabstände.

Anteil der effektiven Spaltbreite für Trimere (3.142) genau dieser Ausdruck als Differenz zur geometrischen Spaltbreite $s_{0}$ auftritt. Für nicht-senkrechten Einfall ist die „Breite" des Trimers auf die Koordinatenrichtung $u_{2}$ abzubilden; dies wird bewirkt durch den Faktor $\left(\cos \phi^{\prime}\right)^{-1}$, wie in Abbildung 3.7 am Beispiel von $\left|r_{\perp}^{(12)}\right|$ dargestellt ist. Somit kann auch die Formel für den geometrischen Anteil der effektiven Spaltbreite (3.128) anschaulich interpretiert werden.

Das für Dimere und Trimere gültige Rezept „effektive Spaltbreite = geometrische Spaltbreite minus Cluster-Breite" (ohne Berücksichtigung der attraktiven Wechselwirkung) gibt Anlaß zur Vermutung, daß es auch bei größeren Clustern gelten könnte. Ein solches Rezept wäre sehr nützlich, da bereits die Formulierung der Beugungstheorie für vieratomige Cluster (Tetramere) um ein Vielfaches aufwendiger als die Berechnungen dieses Kapitels und die Auswertung experimenteller Daten wegen der geringeren Signalstärke weniger genau wären. So könnte zunächst ein Ausdruck für die „Cluster-Breite“ gesucht werden und abgeschätzt werden, ob der gewünschte Nutzen den theoretischen und den experimentellen Aufwand aufwiegt.

\subsubsection{Senkrechter und nicht-senkrechter Einfall}

Die Formeln für die Intensitäten der Beugungsordnungen und die effektiven Spaltbreiten erlauben, den Gewinn an Empfindlichkeit bei der Bestimmung des Paarabstands des Trimers bei nicht-senkrechtem gegenüber senkrechtem Einfall abzuschätzen. Zur Auswertung von Experimenten wird, wie in Kapitel 4 für das Helium-Trimer im Detail beschrieben, die Trimer-Intensitätsformel (3.113) an experimentelle Beugungsintensitäten angepaßt, wobei über den Zwischenschritt der effektiven Spaltbreite der Paarabstand $\langle r\rangle$ als unbekannter Parameter dient. Je stärker die gesamte Anpassung vom Paarabstand abhängt, desto geringer sind seine zu erwartenden Fehlergrenzen. Dagegen wäre die Anpassung ungenau oder gar unmöglich, wenn die Beugungsintensitäten nur schwach oder überhaupt nicht vom Paarabstand abhingen. 
Als ein Maß für die Empfindlichkeit wird hier die relative Änderung der Breite des zentralen Maximums der Einhüllenden des Beugungsbildes in Abhängigkeit vom Paarabstand betrachtet. Diese Wahl gibt ein sowohl für senkrechten als auch für nicht-senkrechten Einfall gleichermaßen verwendbares Kriterium. Unter Vernachlässigung der attraktiven Wechselwirkung beträgt die Breite laut der Intensitätsformel (3.141) bei senkrechtem Einfall in guter Näherung $\Delta P_{2}=4 \pi \hbar / s_{\text {eff }}\left(v^{\prime}\right)$ und laut (3.113) bei nicht-senkrechtem Einfall $\Delta \Pi_{2}=4 \pi \hbar / S_{\text {eff }}\left(v^{\prime}, \overline{\theta^{\prime}}\right)$. Mit Hilfe der Relation (3.101) wird die Breite durch eine anschaulichere Größe, die Anzahl darin enthaltener Beugungsordnungen ausgedrückt: in Analogie zu Kapitel 2 findet man für senkrechten Einfall $n_{c}=d / s_{\text {eff }}\left(v^{\prime}\right)$ und für nicht-senkrechten Einfall $n_{c}=$ $d \cos \overline{\theta^{\prime}} / S_{\text {eff }}\left(v^{\prime}, \overline{\theta^{\prime}}\right) \cos \phi^{\prime}$ Beugungsordnungen zu beiden Seiten der Vorwärtsrichtung ( $n_{c}$ wird hier als kontinuierliche Variable betrachtet). Für die effektiven Spaltbreiten werden nun deren geometrische Anteile eingesetzt. Bei senkrechtem Einfall gilt demnach

$$
n_{c}=\frac{d}{s_{0}-\frac{3}{4}\langle r\rangle}
$$

und mit $S_{0} \cos \phi^{\prime} / \cos \overline{\theta^{\prime}}=s_{0}+t\left(\tan \beta-\tan \overline{\theta^{\prime}}\right)$ bei nicht-senkrechtem Einfall

$$
n_{c}=\frac{d}{s_{0}+t\left(\tan \beta-\tan \overline{\theta^{\prime}}\right)-\frac{3}{4} \frac{\langle r\rangle}{\cos \bar{\theta}^{\prime}}} .
$$

Die relative Änderung von $n_{c}$ läßt sich nun berechnen. Wegen $\langle r\rangle \ll s_{0}$ folgt bis einschließlich der linearen Ordnung für senkrechten Einfall

$$
\frac{1}{n_{c}} \frac{\mathrm{d} n_{c}}{\mathrm{~d}\langle r\rangle}=\frac{3}{4} \frac{1}{s_{0}}\left[1+\frac{3}{4} \frac{\langle r\rangle}{s_{0}}\right]
$$

und für nicht-senkrechten Einfall $\beta<\overline{\theta^{\prime}}$

$$
\frac{1}{n_{c}} \frac{\mathrm{d} n_{c}}{\mathrm{~d}\langle r\rangle}=\frac{3}{4} \frac{1}{\left(s_{0}+t\left(\tan \beta-\tan \overline{\theta^{\prime}}\right)\right) \cos \overline{\theta^{\prime}}}\left[1+\frac{3}{4} \frac{\langle r\rangle}{\left(s_{0}+t\left(\tan \beta-\tan \overline{\theta^{\prime}}\right)\right) \cos \overline{\theta^{\prime}}}\right] .
$$

Einsetzen typischer Zahlenwerte für die Gitterparameter $s_{0}=600 \AA, t=1000 \AA$, $\beta=6^{\circ}$ zeigt, daß die relative Änderung von $n_{c}$ beim mittleren Einfallswinkel $\overline{\theta^{\prime}}=20^{\circ}$ bei $\langle r\rangle=10 \AA$ circa doppelt so groß ist wie bei senkrechtem Einfall. Wegen der komplizierten Form der $\chi^{2}(\langle r\rangle)$-Anpassungsfunktion ist die weitere Analyse nach den Regeln der Fehlerfortpflanzung aufwendig. Da aber $\langle r\rangle / s_{0}$ ein kleiner Parameter ist, sollte auch für sei eine Linearisierung in $\langle r\rangle$ möglich sein. Demnach ist für diese Gitterparameter eine Halbierung der Fehlergrenzen des Paarabstandes gegenüber senkrechtem Einfall zu erwarten. Eine entsprechende Ableitung kann im Rahmen der Atombeugung für die Reduktion der effektiven Spaltbreite durch die van der Waals-Wechselwirkung durchgeführt werden. 


\section{Kapitel 4}

\section{Beugungsexperimente}

Dieses Kapitel befaßt sich mit der Auswertung der im Jahr 2002 von Brühl, Kalinin, Kornilov und Toennies durchgeführten umfangreichen Helium-Beugungsexperimente. Die darin verwendete experimentelle Apparatur (vgl. Abb. 4.1) gleicht prinzipiell derjenigen früherer Experimente [2, 5, 18, 19], wie sie in Ref. [58] ausführlich charakterisiert worden ist. Aus einer Düse mit regelbarer Temperatur und Druck tritt ein Gas von Teilchen aus. Durch einen Skimmer und zwei Kollimationsspalte wird ein Strahl

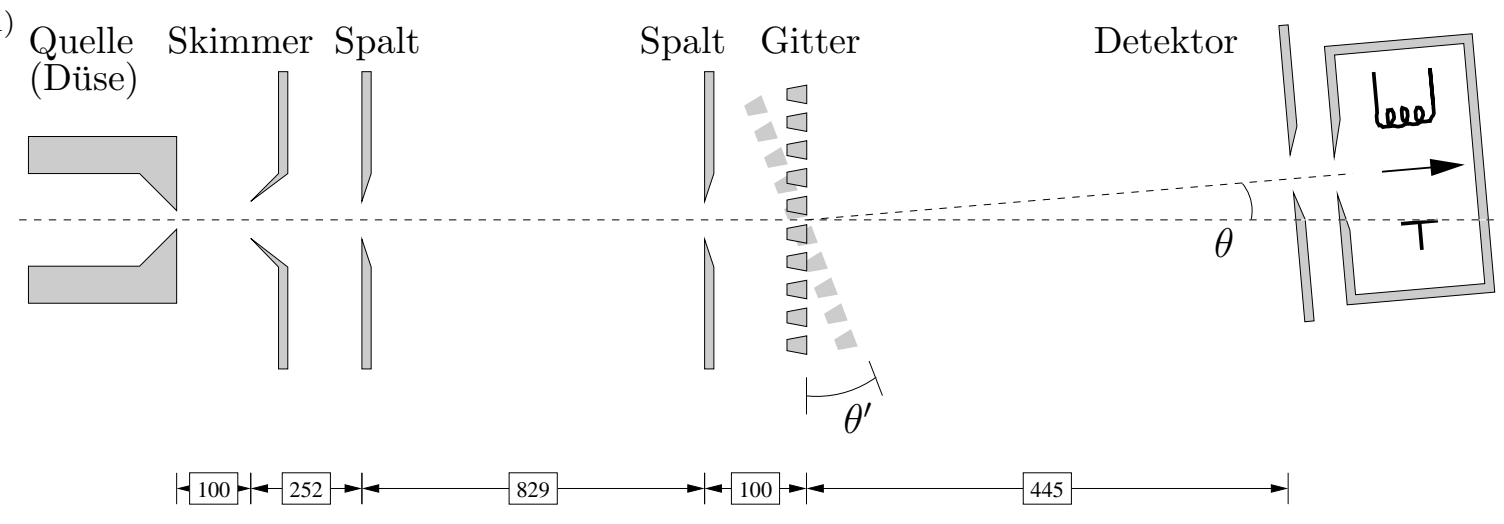

Abbildung 4.1: Skizze des experimentellen Aufbaus der Molekularstrahlapparatur. Die Abstände sind in Millimeter angegeben. Die Kollimationsspalte sind jeweils $20 \mu \mathrm{m}$, der Eingangsspalt des Detektors $25 \mu \mathrm{m}$ breit.

erzeugt, der im Fall von Helium circa $N \approx 100$ Gitterstege kohärent ausleuchtet [18]. Hinter dem Gitter befindet sich ein schwenkbarer Detektor, der aus einer Elektronenionisationsstrecke und einem Massenspektrometer besteht. Im Strahl befinden sich, abhängig von Düsentemperatur und Druck, Helium-Atome und Cluster gleicher Geschwindigkeit aber unterschiedlicher relativer Häufigkeiten, wobei die Anzahl einzelner Atome diejenige der Cluster immer weit übersteigt [56, 57]. Allgemein lassen sich aufgrund der massenabhängigen de Broglie-Wellenlänge $\lambda_{\mathrm{dB}}=\hbar / M v^{\prime}$ die unter den Beugungswinkeln (3.102) auftretenden Beugungsmaxima Clustern bestimmter Masse $M$ eindeutig zuordnen. Unter gleichen Winkeln auftretende Beugungsmaxima verschiedener Cluster können jedoch nicht ausgewertet werden; dies ist beispielsweise für die Ordnungen $n=0, \pm 3, \pm 6, \ldots$ von ${ }^{4} \mathrm{He}_{3}$ der Fall, die mit den Ordnungen 
$n=0, \pm 1, \pm 2, \ldots$ von ${ }^{4}$ He zusammenfallen. Um reine Atom-Beugungsbilder aufnehmen zu können, wird der Cluster-Anteil so gering wie möglich eingestellt. Abbildung 4.2 zeigt ein typisches Beugungsbild, das für eine hohe Anzahl von Helium-Trimeren optimiert wurde.

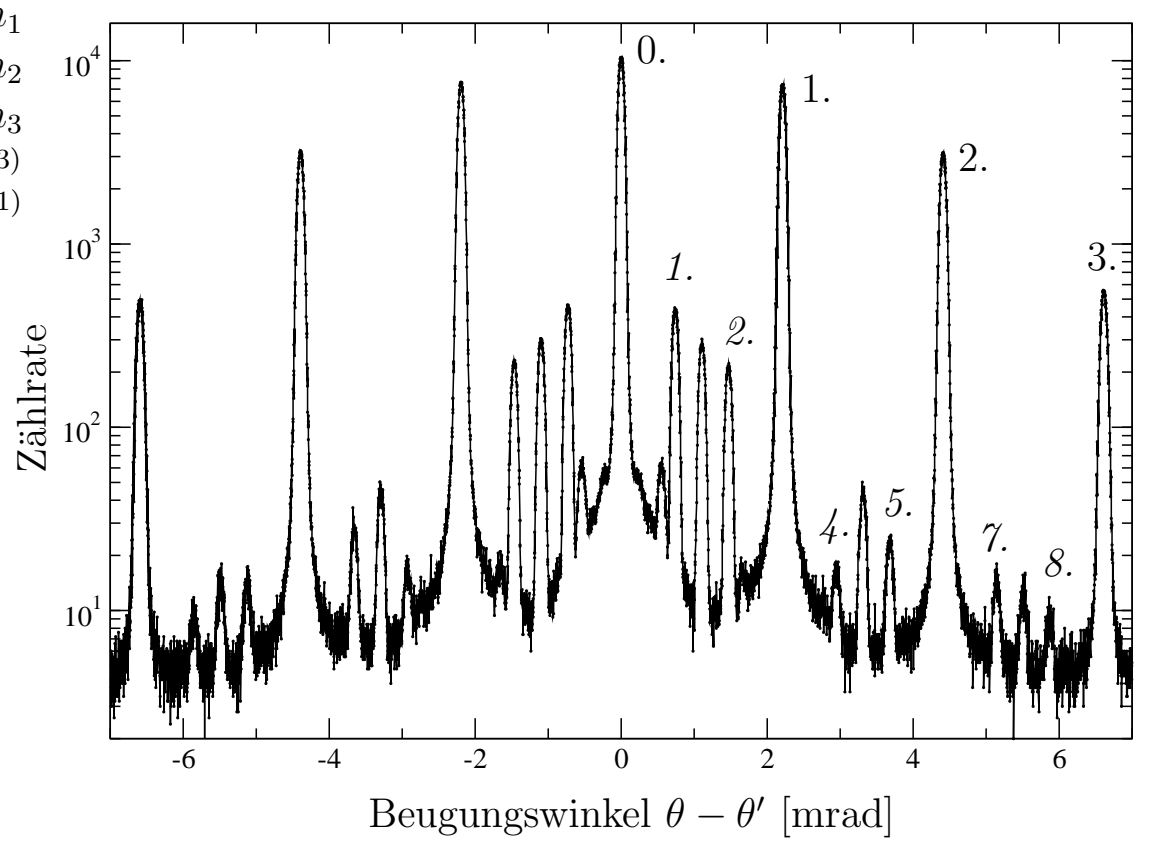

Abbildung 4.2: Auf einen hohen Anteil von Trimeren optimiertes experimentelles Beugungsbild eines Helium-Strahls bei einem Einfallswinkel von $\theta^{\prime}=21^{\circ}$ und einer Strahlgeschwindigkeit von $v^{\prime}=484,1 \mathrm{~ms}^{-1}$ aus den Experimenten von Brühl, Kalinin, Kornilov und Toennies. Die intensiven Beugungsmaxima rühren von einzelnen Helium-Atomen her, die kursiv bezeichneten Beugungsmaxima von Trimeren. Auf halbem Abstand zwischen zwei Atom-Maxima ist jeweils noch ein Beugungsmaximum der Dimere sichtbar.

Die drei Beugungsexperimente wurden bei den Einfallswinkeln $\theta^{\prime}=18^{\circ}, \theta^{\prime}=21^{\circ}$ und $\theta^{\prime}=24^{\circ}$ durchgeführt. Jedes dieser Experimente besteht aus Meßserien für Atome, Dimere und Trimere bei gleicher Gittergeometrie; die Parameter Düsentemperatur und Düsendruck wurden jeweils auf die optimale Produktionsrate für die gewünschte Spezies eingestellt. Eine Meßserie besteht aus vier bis sieben bei verschiedenen Strahlgeschwindigkeiten aufgenommenen Beugungsbildern. Die Strahlgeschwindigkeit wurde jeweils mit Hilfe eines Tabellenwerkes [61] aus den Parametern Düsentemperatur und Druck interpoliert.

Die Auswertung der Beugungsexperimente verläuft nach einem festen Schema. Zunächst wird anhand von atomaren Beugungsdaten die Gittergeometrie charakterisiert. Anschließend werden die geometrischen Parameter festgehalten und die Meßserien für Dimere und Trimere ausgewertet. 


\subsection{Transmissionsmessung}

Der erste Schritt zur Auswertung besteht in der Charakterisierung der Gittergeometrie. Als „Transmissionsmessung“" wird hier die Messung der Intensität der nullten Beugungsordnung in Abhängigkeit vom Einfallswinkel bezeichnet. Sie erlaubt zum einen die Justage des Gitters und zum anderen die Bestimmung des Gitterwinkels $\varphi$. Wegen der trigonometrischen Identität $\sin \left(\varphi-\theta^{\prime}\right) / \cos \theta^{\prime}=\left(\tan \varphi-\tan \theta^{\prime}\right) \cos \varphi$ lautet die Intensität der nullten Beugungsordnung für Einfallswinkel $\beta<\theta^{\prime}<\varphi$ der atomaren Intensitätsformel (2.75) zufolge

$$
I_{0} \propto\left(\tan \varphi-\tan \theta^{\prime}\right)^{2} \cos ^{2} \varphi \frac{S_{\mathrm{eff}}^{2}\left(v^{\prime}, \theta^{\prime}\right)+\Delta^{2}\left(v^{\prime}, \theta^{\prime}\right)}{d^{2}}
$$

wobei der experimentell vorgegebene Einfallswinkel mit $\theta^{\prime}$ anstelle von $\overline{\theta^{\prime}}$ bezeichnet wurde. Der Faktor $\left(\tan \varphi-\tan \theta^{\prime}\right)$ reflektiert die Tatsache, daß das Gitter beim Einfallswinkel $\theta^{\prime}=\varphi$ schließt. Die in Abschnitt A.1.3 abgeleiteten asymptotischen Relationen für die atomaren Kumulanten $R_{1}^{ \pm}$erlauben, Gleichung (4.1) bei hohen Geschwindigkeiten zu nähern:

$$
\sqrt{I_{0}} \propto\left(\tan \varphi-\tan \theta^{\prime}\right) \cos \varphi \frac{S_{0}}{d} \sqrt{1-\frac{\sqrt{2 \pi}}{S_{0}}\left(\sqrt{\lambda_{+}}+\sqrt{\lambda_{-}}\right) .}
$$

Die vom Einfallswinkel und den Gitterparametern abhängigen Größen $\lambda_{ \pm}$, die in Gleichung (A.11) definiert werden, repräsentieren dabei die durch die attraktive Wechselwirkung bedingte Korrektur. Exakte Zahlenwerte für $\lambda_{ \pm}$können zu diesem Zeitpunkt der Auswertung noch nicht berechnet werden, da nicht alle Parameter bekannt sind. Einsetzen typischer Werte für Helium-Atome im Grundzustand zeigt allerdings, daß diese Korrektur sehr klein ist: Die durch Vernachlässigung der Korrektur erhaltene einfache Formel

$$
\sqrt{I_{0}} \propto\left(\tan \varphi-\tan \theta^{\prime}\right) \cos \varphi \frac{S_{0}}{d}
$$

führt zu einer Unterschätzung des Winkels $\varphi$ um nur circa $1 \%$ bei der experimentellen Auswertung.

Im Rahmen der Gültigkeit dieser Näherung kann auch der Keilwinkel $\beta$ des Gitters aus der Transmissionsmessung bestimmt werden. Dazu beobachtet man, daß die Intensität der nullten Beugungsordnung des Gitters für Einfallswinkel $0 \leq \theta^{\prime}<\beta$ unabhängig von $\theta^{\prime}$ ist. Der Übergang von konstanter Intensität in den von $\tan \theta^{\prime}$ anhängigen Ausdruck (4.3) findet bei $\theta^{\prime}=\beta$ statt.

Abbildung 4.3 zeigt eine experimentelle Transmissionskurve, deren Nullpunktsverschiebung bereits korrigiert wurde. An die Flanken wurden Regressionsgeraden (4.3) angepaßt. Die Schnittpunkte der Regressionsgeraden mit der Einfallswinkelachse liegen bei $\theta^{\prime}= \pm \varphi$. Für die in Abbildung 4.3 dargestellte Messung erhält man $\varphi=0,56197 \pm 0,0007 \mathrm{rad}$ entsprechend 32,20 $\pm 0,04^{\circ}$. Die Genauigkeit von $\varphi$ wird also stärker durch die Approximation (4.3) beeinflußt als durch die Meßunsicherheiten. Der Keilwinkel $\beta$ kann aus den Schnittpunkten der Regressionsgeraden mit der um $\theta^{\prime}=0$ angepaßten konstanten Funktion abgelesen werden. Man findet hiernach $\beta=6,5 \pm 0,1^{\circ}$. 


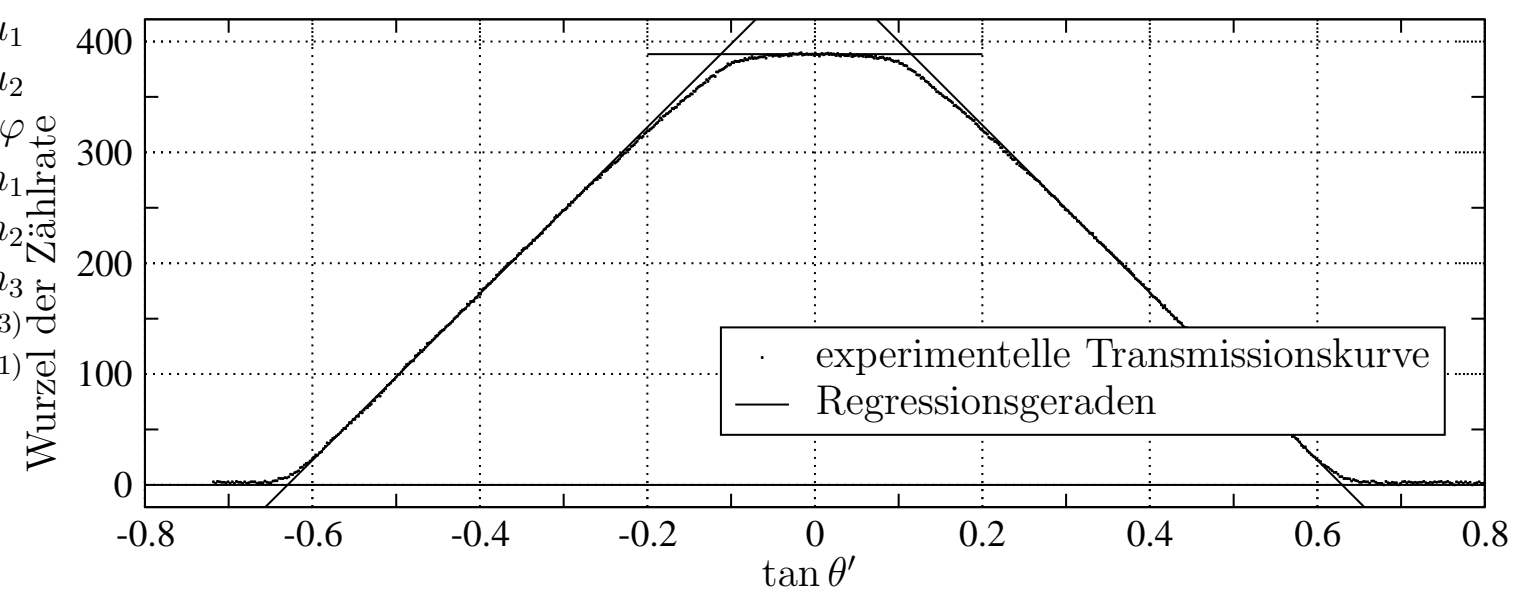

Abbildung 4.3: Auswertung einer experimentellen Transmissionskurve bei $v^{\prime}=1780 \mathrm{~m} / \mathrm{s}$. Aufgetragen ist die Wurzel der experimentellen Zählrate gegen den Tangens des Einfallswinkels. Ebenso sind Regressionsgeraden an die linke und rechte Flanke der Kurve nach Gleichung (4.3) eingetragen. Die Schnittpunkte mit der Abszisse liefern den Winkel $\varphi$. Die Schnittpunkte mit der um $\theta^{\prime}=0$ angepaßten konstanten Funktion liefern den Keilwinkel $\beta$.

\subsection{Atom-Beugungsbilder}

Nun können die atomaren Beugungsbilder zur Bestimmung der geometrischen Spaltbreiten $s_{0}$ beziehungsweise $S_{0}$ und der Konstanten der attraktiven Wechselwirkung $C_{3}$ ausgewertet werden ${ }^{1}$. Dazu wird an jedes der Beugungsbilder zu senkrechtem Einfall die kumulantenentwickelte Intensitätsformel (2.81) über die Parameter $s_{\text {eff }}, \delta$ und $\sigma$ angepaßt; zu nicht-senkrechtem Einfall wird stattdessen die Intensitätsformel (2.75) mit den Parametern $S_{\text {eff }}, \Delta, \Sigma$ und $\alpha$ verwendet. In Abbildung 4.4 ist die Anpassung zweier atomarer Beugungsbilder bei $\theta^{\prime}=0^{\circ}$ und $\theta^{\prime}=21^{\circ}$ dargestellt. Wegen der in der nullten Beugungsordnung anteilsmäßig in größter Zahl enthaltenen unerwünschten Cluster wurde diese mit einer verhundertfachten Fehlerangabe versehen.

Aus jedem Beugungsbild wird somit ein Zahlenwert für die effektive Spaltbreite $s_{\text {eff }}$ beziehungsweise $S_{\text {eff }}$ bestimmt. Für jede Meßserie, die aus Beugungsbildern bei mehreren Geschwindigkeiten besteht, werden anschließend die experimentellen effektiven Spaltbreiten zur Anpassung von $s_{0}$ beziehungsweise $S_{0}$ und $C_{3}$ mit den theoretischen Formeln $s_{\text {eff }}\left(v^{\prime}\right)(2.82)$ beziehungsweise $S_{\text {eff }}\left(v^{\prime}, \overline{\theta^{\prime}}\right)(2.76)$ verglichen. Die in Abschnitt A.1.3 abgeleiteten asymptotischen Relationen zwischen den effektiven Spaltbreiten und der Strahlgeschwindigkeit erlauben dabei die Identifikation von Meßausreißern. In Abbildung 4.5 sind die Zahlenwerte für $s_{\text {eff }}$ beziehungsweise $S_{\text {eff }}$ aus der Anpassung der Meßserien zu senkrechtem Einfall und zu $\theta^{\prime}=21^{\circ}$ dargestellt. Außerdem sind die nach den Gleichungen (2.82) beziehungsweise (2.76) angepaßten theoretischen Kurven eingetragen. Die Werte für die geometrischen Spaltbreiten $s_{0}$ und $S_{0}$ können direkt als Achsenabschnitte in Abbildung 4.5 abgelesen werden. Für

\footnotetext{
${ }^{1}$ Die Größen $\lambda_{ \pm}$(A.11) sind proportional zur Wechselwirkungskonstanten $C_{3}$.
} 

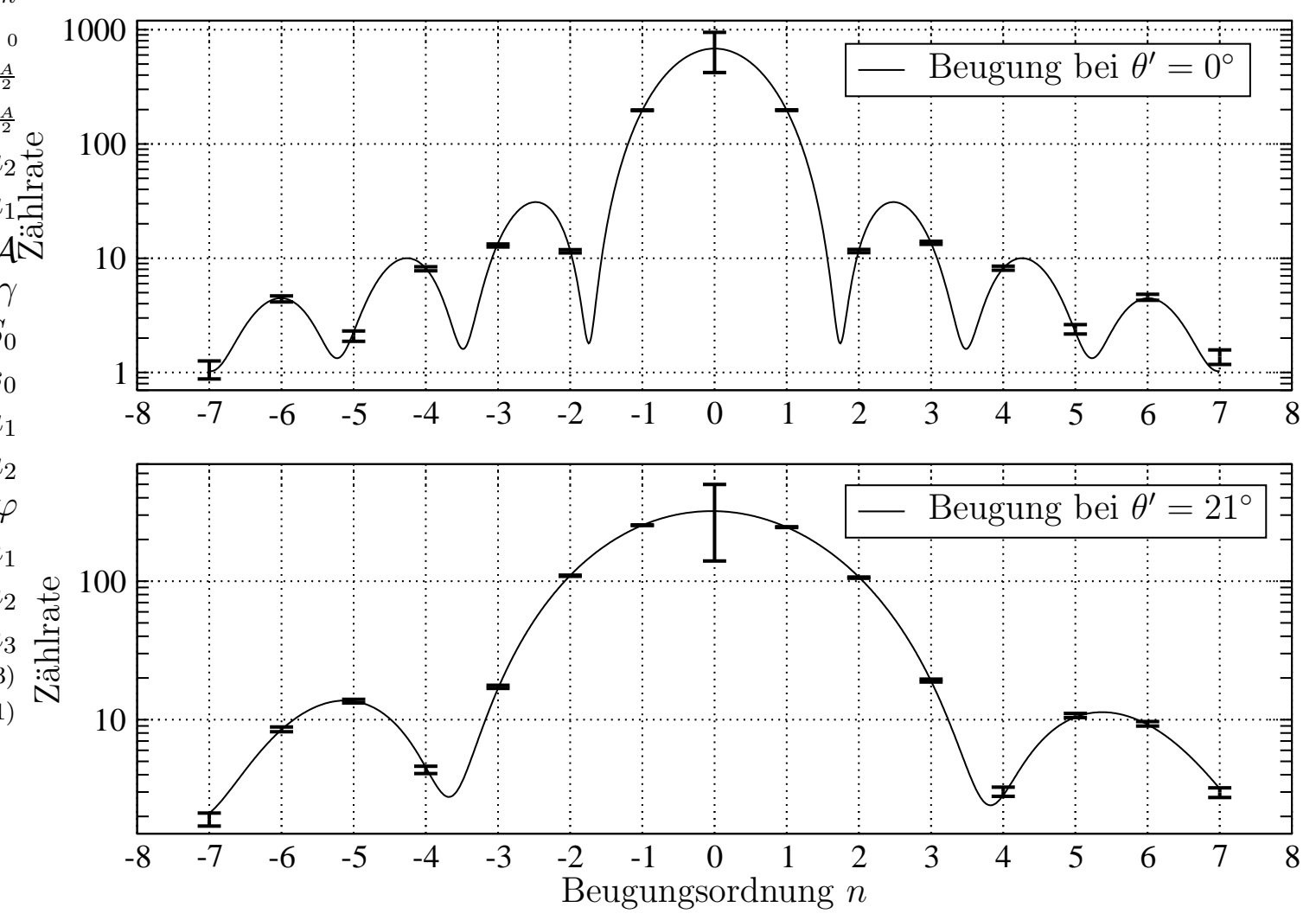

Abbildung 4.4: Experimentelle Beugungsintensitäten für Atome bei $\theta^{\prime}=0^{\circ}$ (oben) und $\theta^{\prime}=21^{\circ}$ (unten) bei der Geschwindigkeit $v^{\prime}=494,9 \mathrm{~m} / \mathrm{s}$ sowie Anpassungen der Intensitätsformeln (2.81) bzw. (2.75). Da die senkrechte Projektion der Spaltbreite $S_{0}$ auf die Einfallsrichtung bei $21^{\circ}$ circa um den Faktor 2,2 kleiner ist als $s_{0}$, erscheint das untere Beugungsbild um diesen Faktor gestreckt. 

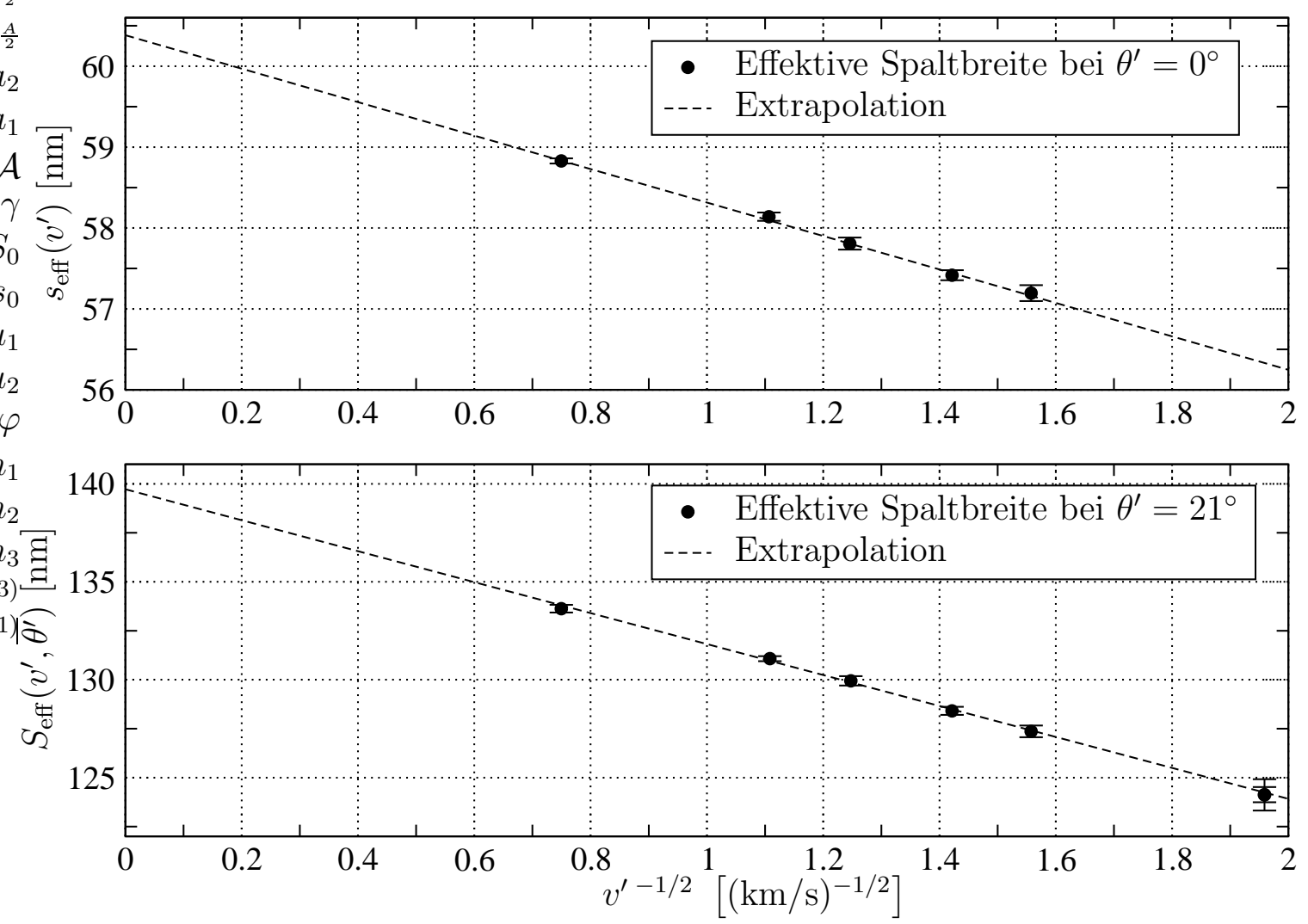

Abbildung 4.5: Aus den Meßserien zu senkrechtem (oben) und zu nicht-senkrechtem Einfall bei $\theta^{\prime}=21^{\circ}$ (unten) ermittelte Zahlenwerte $s_{\text {eff }}$ beziehungsweise $S_{\text {eff }}$ und theoretische Kurven für die effektiven Spaltbreiten. 
$C_{3}$ muß die Anpassung numerisch durchgeführt werden. Aus Abbildung 4.5 ist allerdings zu erkennen, daß die asymptotischen Relationen im benötigten Geschwindigkeitsbereich bereits eine sehr gute Beschreibung liefern, sodaß $C_{3}$ zur Kontrolle auch aus der Steigung berechnet werden kann.

Entsprechende Auswertungen wurden für die Experimente bei den Einfallswinkeln $\theta^{\prime}=18^{\circ}$ und $24^{\circ}$ durchgeführt. Ihre effektiven Spaltbreiten sind in den Abbildungen 4.6 und 4.7 dargestellt.
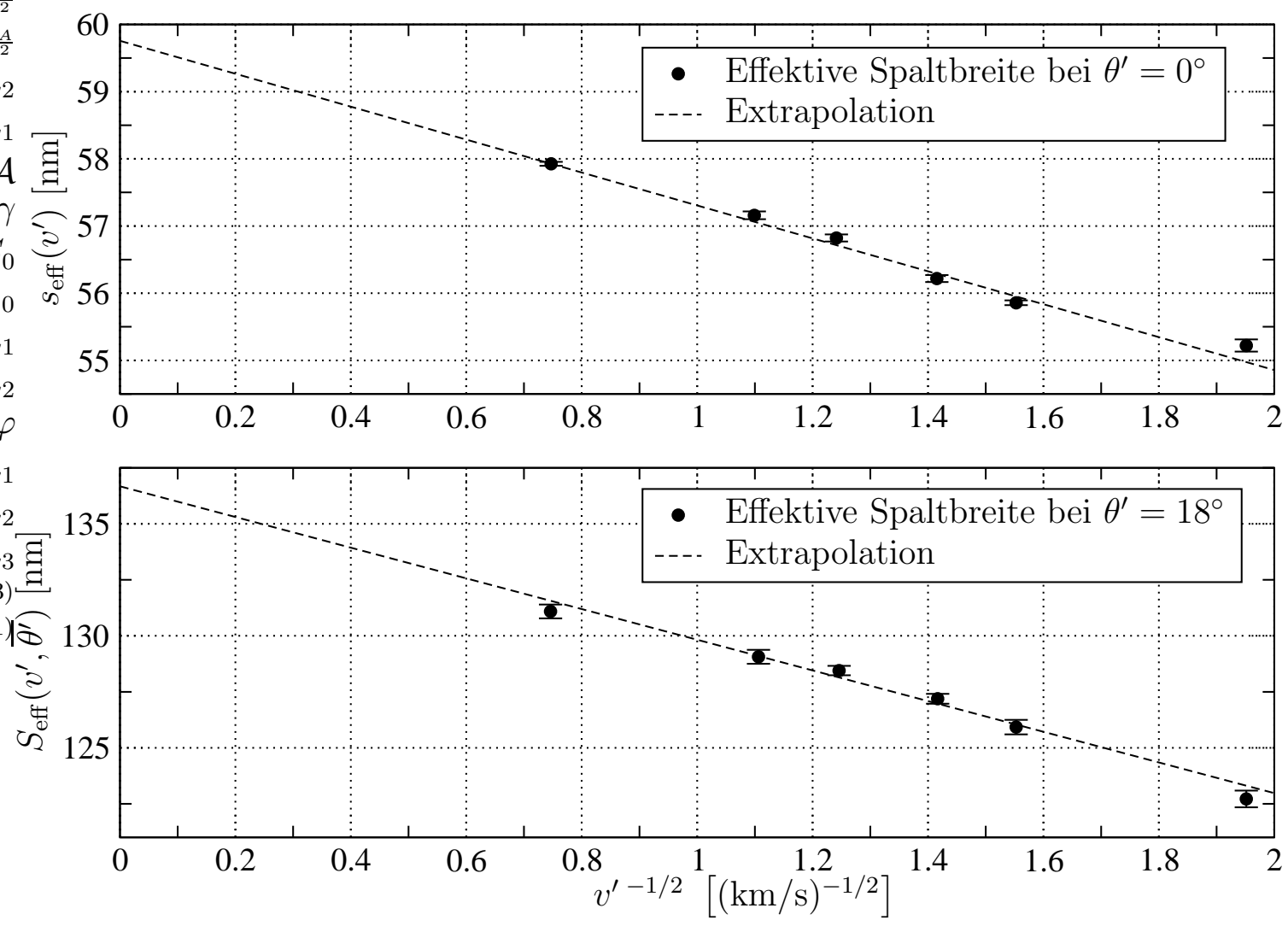

Abbildung 4.6: wie Abb. 4.5 aber für das Experiment zu $\theta^{\prime}=18^{\circ}$.

In Tabelle 4.1 sind die Ergebnisse der Auswertung aller atomaren Transmissionsmessungen und Beugungsbilder zu senkrechtem und nicht-senkrechtem Einfall zusammengefaßt. Es ist auffällig, daß die Wechselwirkungskonstante $C_{3}$ zwischen den unterschiedlichen Experimenten stärker schwankt, als es die einzelnen Fehlerangaben erwarten lassen, obwohl das Gitter jeweils aus dem gleichen Material bestand (Silizium-Nitrid) und für die Experimente zu $18^{\circ}$ und $21^{\circ}$ sogar dasselbe Gitter verwendet wurde. Der tatsächliche Fehler von $C_{3}$ ist daher wahrscheinlich nicht von der Größenordnung der in Tabelle 4.1 angegebenen Grenzen; ein Maß erhält man aber aus der Schwankung zwischen den Meßserien. Um eine einheitliche Auswertung zu ermöglichen, wird für $C_{3}$ im folgenden der Mittelwert aller bisher (z. T. unveröffentlichten) von Grisenti, Schöllkopf und Toennies [5] und Brühl, Kalinin, Kornilov und Toennies an Silizium-Nitrid-Gittern durchgeführten Meßserien einschließlich der Daten aus Tabelle 4.1 verwendet. Dieser Mittelwert beträgt $C_{3}:=0,113 \pm 0,020 \mathrm{meV}$ $\mathrm{nm}^{3}$. 


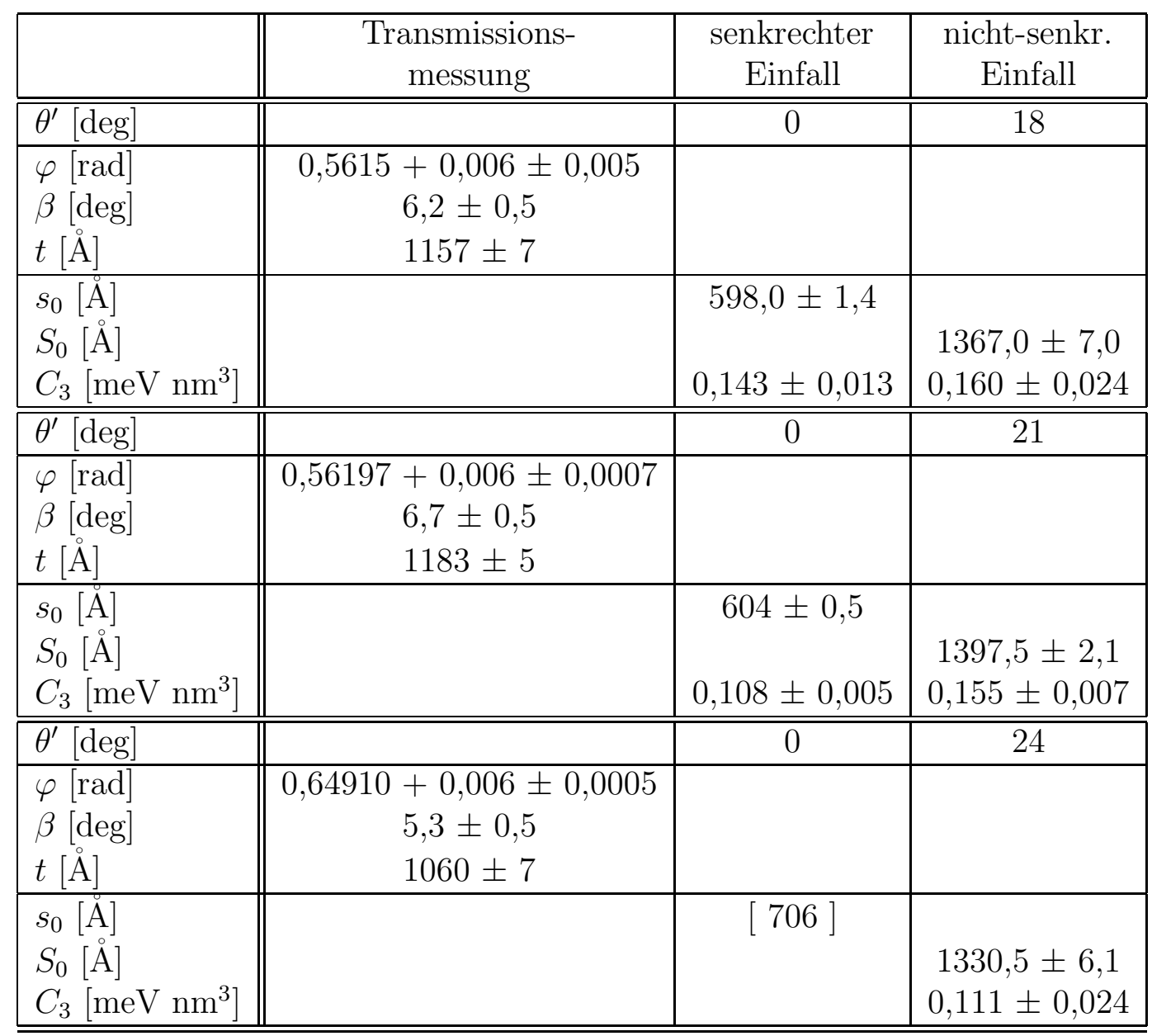

Tabelle 4.1: Ergebnisse der Auswertung der atomaren Transmissionsmessungen und Beugungsbilder. Wegen der auf Seite 79 diskutierten Unterschätzung des Winkels $\varphi$ um circa $1 \%$ bei Auswertung der Transmissionsmessungen wurde seine Fehlerangabe nach oben vergrößert. Die Gittertiefe $t$ wurde aus der Relation $t=S_{0} \cos \varphi$ berechnet. Der Keilwinkel $\beta$ wurde sowohl aus den Transmissionsmessungen als auch aus der geometrischen Relation $\tan \beta=\tan \varphi-s_{0} / t$ bestimmt. Beide Ergebnisse stimmen im Rahmen der angegebenen Fehlergrenzen überein. Zu der Messung mit $\theta^{\prime}=24^{\circ}$ wurde keine Vergleichsmessung bei senkrechtem Einfall durchgeführt; die in eckigen Klammern angegebene Spaltbreite $s_{0}$ wurde aus $S_{0}$ und $\varphi$ berechnet. 


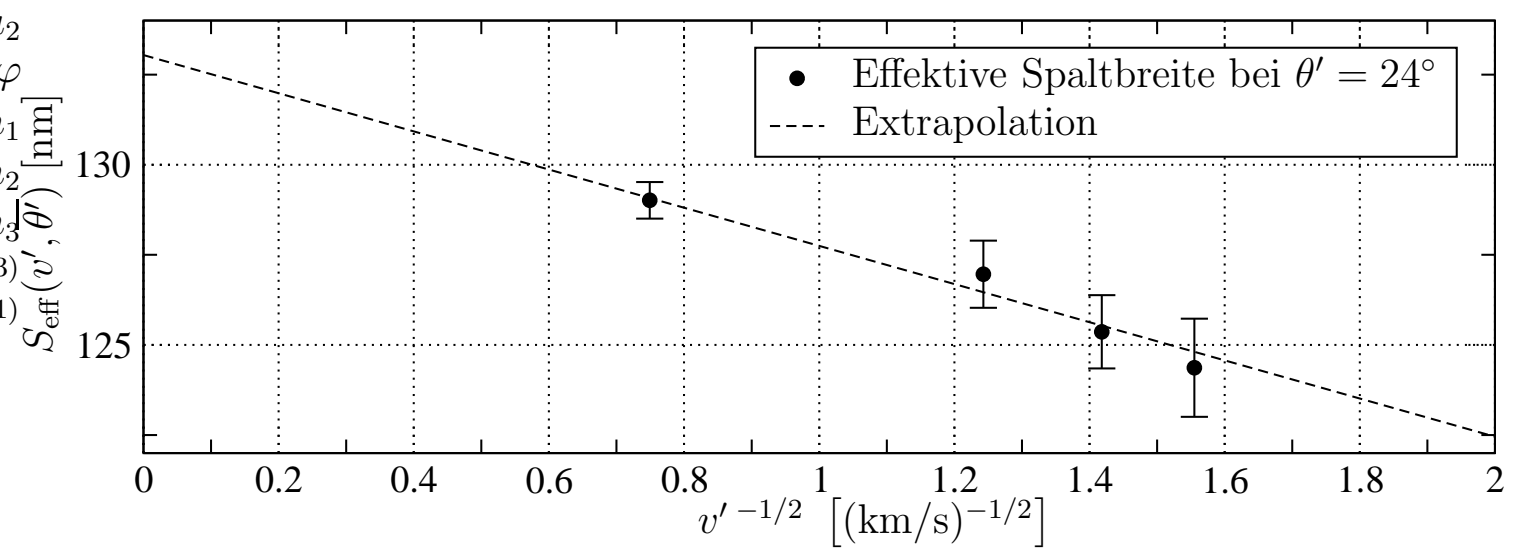

Abbildung 4.7: wie Abb. 4.5 aber für das Experiment zu $\theta^{\prime}=24^{\circ}$. Es liegt keine Vergleichsmessung zu senkrechtem Einfall vor.

Die Vorgabe von $C_{3}$ legt in den Abbildungen 4.5-4.7 die Steigungen der theoretischen Kurven fest; sie passen also in den speziellen Fällen weniger gut die Werte der effektiven Spaltbreiten an. Generell sollten aber Werte für hohe Strahlgeschwindigkeiten verläßlicher sein als für niedrige Geschwindigkeiten, da die attraktive Wechselwirkung proportional zu $v^{-1 / 2}$ abnimmt. Aus diesem Grund wurde die für folgende Auswertung eine zusätzliche Wichtung der Werte der effektiven Spaltbreiten durch lineare Vergrößerung der experimentellen Fehlerbalken mit abnehmender Geschwindigkeit vorgenommen: der Fehlerbalken der zweitschnellsten Messung wurde verdoppelt, derjenige der drittschnellsten verdreifacht usw. Die Neuauswertung der geometrischen Spaltbreiten ist in Tabelle 4.2 zusammengefaßt.

\begin{tabular}{|c|c|c|}
\hline & $\begin{array}{l}\text { senkrechter } \\
\text { Einfall }\end{array}$ & $\begin{array}{l}\text { nicht-senkr. } \\
\text { Einfall }\end{array}$ \\
\hline$\overline{\theta^{\prime}[\mathrm{deg}]}$ & $\begin{array}{l}0 \\
\end{array}$ & $\begin{array}{ll}18 \\
\end{array}$ \\
\hline $\begin{array}{l}s_{0}[\stackrel{\mathrm{A}}{]}] \\
S_{0}[\AA] \AA\end{array}$ & $595,0 \pm 0,6$ & $1354,0 \pm 0,9$ \\
\hline$\overline{\overline{\theta^{\prime}[\mathrm{deg}]}}$ & 0 & 21 \\
\hline $\begin{array}{l}s_{0}[\AA] \AA \\
S_{0}[\AA]\end{array}$ & $604,5 \pm 0,2$ & $1386,0 \pm 0,7$ \\
\hline$\overline{\overline{\theta^{\prime}[\mathrm{deg}]}}$ & $\overline{0}$ & $\begin{array}{l}24 \\
\end{array}$ \\
\hline $\begin{array}{l}s_{0}[\ddot{\mathrm{A}}] \\
S_{0}[\check{\AA}]\end{array}$ & 706 & $1330,5 \pm 0,9$ \\
\hline
\end{tabular}

Tabelle 4.2: Ergebnisse der Auswertung der atomaren Beugungsbilder für die geometrischen Spaltbreiten bei festgelegtem $C_{3}=0,113 \mathrm{meV} \mathrm{nm}^{3}$. Die anderen Parameter können aus Tabelle 4.1 übernommen werden. 


\subsection{Trimer-Beugungsbilder}

Den Ergebnissen von Kapitel 3 zufolge besteht ein Beugungsbild von Helium-Trimeren grundsätzlich aus der inkohärenten Überlagerung der Beugungsbilder der beiden Bindungszustände. Allerdings ist die Besetzungswahrscheinlichkeit des angeregten Trimer-Zustandes sehr klein, wie die folgende Abschätzung zeigt: Bei einer für einen Heliumstrahl typischen Geschwindigkeitsverteilung von $\Delta v^{\prime} / v^{\prime} \approx 2 \%$ [18] und der mittleren Strahlgeschwindigkeit $v^{\prime}=500 \mathrm{~ms}^{-1}$ beträgt die Temperatur im mitbewegten System des Teilchenstrahls $T_{b}=m\left(\Delta v^{\prime}\right)^{2} / 2 k_{\mathrm{B}} \approx 27 \mathrm{mK}$ [62], wobei $k_{\mathrm{B}}$ die Boltzmann-Konstante bezeichnet. Unter der Annahme, daß die Besetzungszahlen der beiden Trimer-Zustände jeweils proportional zum Boltzmann-Faktor $\exp \left(-E_{\gamma} / k_{\mathrm{B}} T_{b}\right)$ sind, wobei die Bindungsenergien $E_{\gamma}$ aus Tabelle 3.1 entnommen werden können, liegen nur circa 1\% der Helium-Trimere im angeregten Zustand vor. Da die Beugungsordnungen beider Zustände unter denselben Winkeln auftreten, sollte der Beitrag des angeregten Zustandes nicht auflösbar sein. Darüberhinaus sollte die Anregungswahrscheinlichkeit des Grundzustandes bei der Wechselwirkung mit dem Gitter vernachlässigbar klein sein [63]. Im folgenden wird daher angenommen, daß die experimentellen Beugungsbilder allein von elastischer Beugung des Grundzustandes von ${ }^{4} \mathrm{He}_{3}$ herrühren.

$\mathrm{Zu}$ jeder Meßserie von Trimer-Beugungsbildern wurde im letzten Unterabschnitt mit Hilfe der zugehörigen Atom-Beugungsbilder die Gittergeometrie charakterisiert. Als freier Parameter verbleibt nurmehr der mittlere Paarabstand $\langle r\rangle_{\mathrm{g}}$ des Grundzustandes, der in die effektive Spaltbreite für Trimere eingeht. Zur experimentellen Bestimmung der effektiven Spaltbreite der Trimere wird die kumulantenentwickelte Intensitätsformel (3.113) an alle Trimer-Beugungsbilder einer Meßserie angepaßt. In Abbildung 4.8 ist eine solche Anpassung dargestellt.

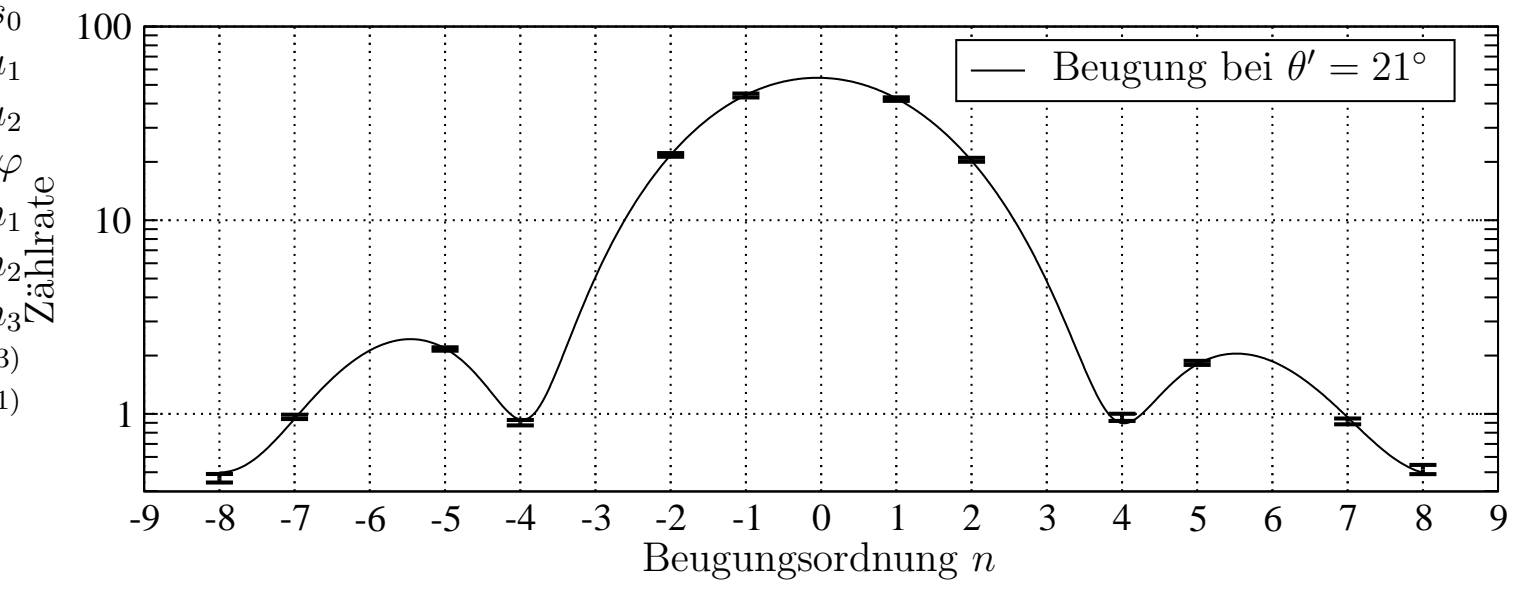

Abbildung 4.8: Ausgewertete Trimer-Beugungsintensitäten des Beugungsbildes aus Abbildung 4.2 bei $\theta^{\prime}=21^{\circ}$ bei der Geschwindigkeit $v^{\prime}=484,1 \mathrm{~ms}^{-1}$. Die Beugungsordnungen $n=0, \pm 3, \pm 6$ fehlen im Spektrum, da sie mit atomaren Beugungsordnungen zusammenfallen und nicht ausgewertet werden können. Die durchgezogene Kurve stellt eine Anpassung der kumulantenentwickelten Intensitätsformel (3.113) dar.

In Abbildung 4.9 sind sämtliche Werte für die effektive Spaltbreite des Trimers 
für die Meßserie zu $\theta^{\prime}=21^{\circ}$ zusammengefaßt. Die beste Anpassung der theoretischen Formel (3.135/3.138) erfolgt bei dem Paarabstand $\langle r\rangle_{\mathrm{g}}=10,0 \AA$. Um zu einer
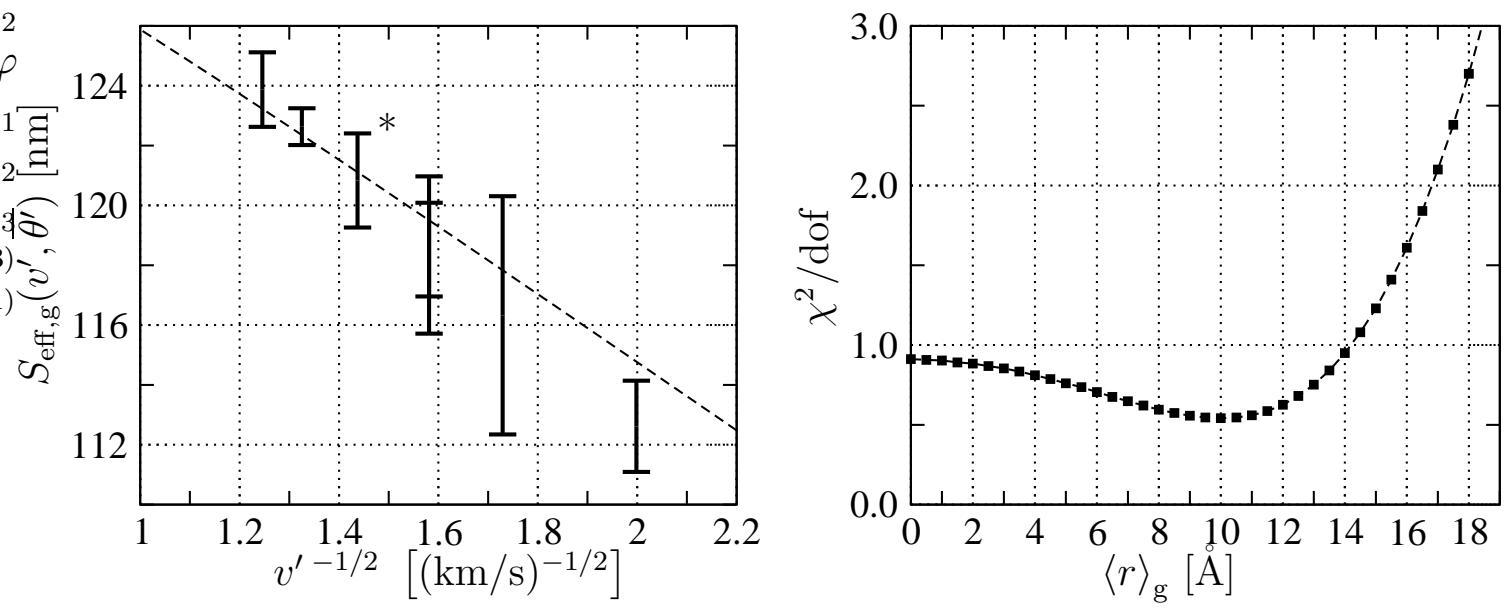

Abbildung 4.9: Links: Aus der Meßserie zu $\theta^{\prime}=21^{\circ}$ ermittelte sieben Zahlenwerte bei sechs verschiedenen Geschwindigkeiten für die effektive Spaltbreite für Trimere. Die mit einem Stern markierte Messung entspricht dem Beugungsbild aus den Abbildungen 4.2 und 4.8. Die gestrichelte Kurve stellt eine Anpassung der approximierten Gleichung für die effektive Spaltbreite (3.135/3.138) an die gewichteten Daten (vgl. S. 83) dar, wobei der mittlere Paarabstand $\langle r\rangle_{\mathrm{g}}$ als Anpassungsparameter dient. Die beste Anpassung wird erreicht bei dem Wert $\langle r\rangle_{\mathrm{g}}=10,0 \AA$. Rechts: Numerisch berechnete „Chiquadrat pro Freiheitsgrad“Funktion zur Minimierung der mittleren quadratischen Abweichung zwischen experimentellem und theoretischem Ergebnis bei der Anpassung im linken Bild. Das Minimum liegt bei dem genannten Wert von $\langle r\rangle_{\mathrm{g}}$.

Fehlerabschätzung dieses Ergebnisses zu gelangen, wurde die gesamte Auswertung in verschiedenen Kombinationen der eingehenden, fehlerbehafteten Parameter $\varphi, \beta, C_{3}$ und $S_{0}$ durchgeführt. Dabei stellte sich heraus, daß die Streuung im wesentlichen von den experimentellen Unsicherheiten der Größen $C_{3}$ und $S_{0}$ herrührt. Für die in Abbildung 4.9 dargestellte Meßserie gilt laut Tabelle 4.2 beispielsweise $S_{0}=1386,0 \pm 0,7 \AA$ und $C_{3}=0,113 \pm 0,020 \mathrm{meV} \mathrm{nm}^{3}$. Die Unsicherheit von $S_{0}$ führt bei festgehaltenem $C_{3}$ auf den Fehler $\pm 0,5 \AA$ in $\langle r\rangle_{\mathrm{g}}$. Die Unsicherheit von $C_{3}$ führt bei festgehaltenem $S_{0}$ auf den Fehler $+6,5 /-9,5 \AA$ in $\langle r\rangle_{\mathrm{g}}$. Der auf Seite 71 diskutierte Approximationsfehler ist dagegen vernachlässigbar klein. Als gesamter Fehler von $\langle r\rangle_{\mathrm{g}}$ wird im folgenden die Wurzel der mittleren quadratischen Abweichung angegeben, im vorliegenden Fall also $\langle r\rangle_{\mathrm{g}}=10,0+5 /-7 \AA$.

Eine entsprechende Auswertung wurde für die Meßserien zu $\theta^{\prime}=18^{\circ}$ und $24^{\circ}$ durchgeführt. In den Abbildungen 4.10 und 4.11 sind die effektiven Spaltbreiten und ihre Anpassungen dargestellt. Als Ergebnisse erhält man $\langle r\rangle_{\mathrm{g}}=12,0+5 /-8 \AA$ aus der Messung zu $\theta^{\prime}=18^{\circ}$ und $\langle r\rangle_{\mathrm{g}}=18,5 \pm 2,5 \AA$ aus der Messung zu $\theta^{\prime}=24^{\circ}$.

In Tabelle 4.3 sind die Ergebnisse der drei Experimente zusammengefaßt. Wegen der relativ großen Abweichung des bei $\theta^{\prime}=24^{\circ}$ gemesssenen Paarabstandes von den beiden anderen Resultaten ist deren Beurteilung zu diskutieren. Die Messungen bei 

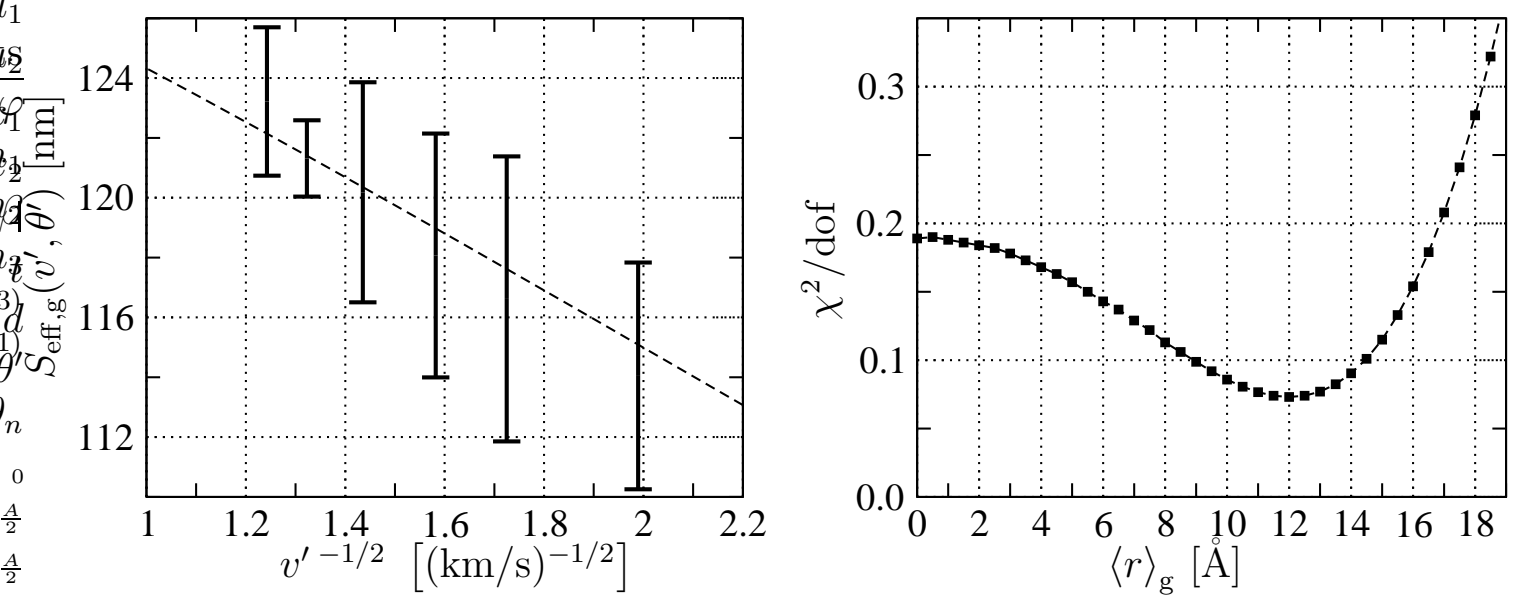

Abbildung 4.10: Links: Aus der Meßserie zu $\theta^{\prime}=18^{\circ}$ ermittelte sechs Zahlenwerte bei sechs verschiedenen Geschwindigkeiten für die effektive Spaltbreite für Trimere. Die beste Anpassung wird erreicht bei dem Wert $\langle r\rangle_{\mathrm{g}}=12,0 \AA$. Rechts: Chiquadrat-Funktion.
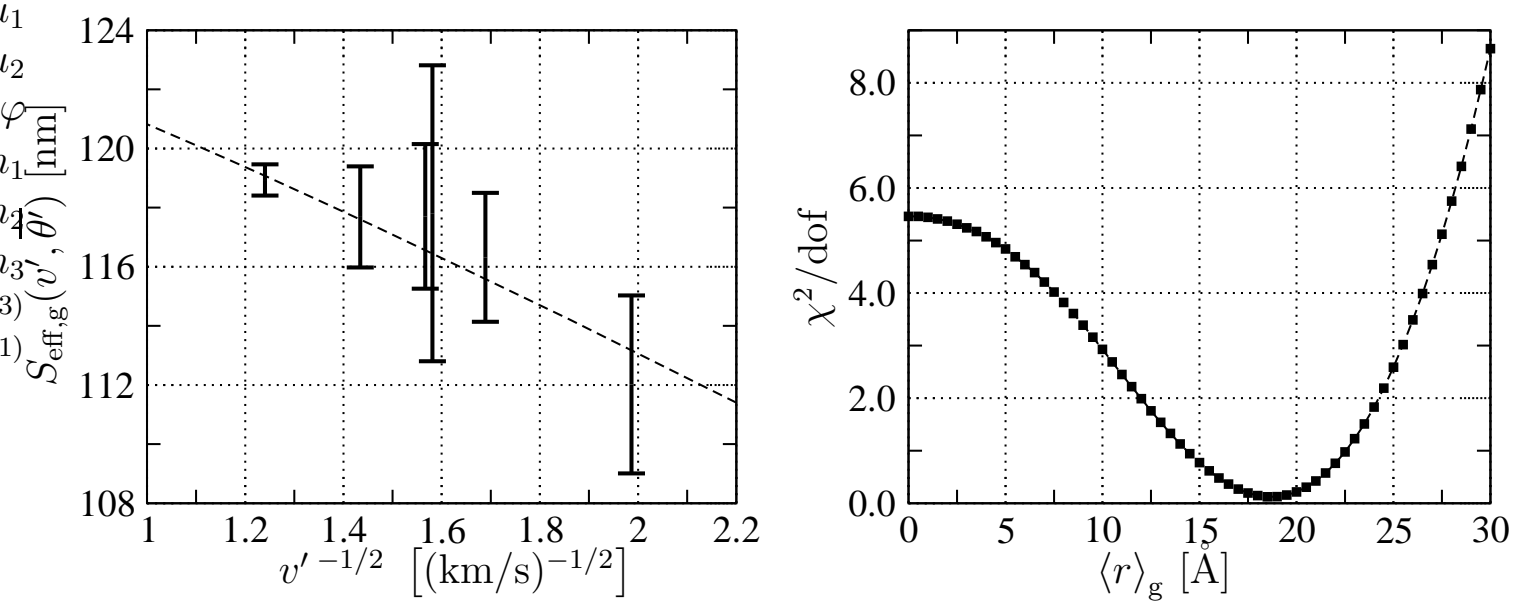

Abbildung 4.11: Links: Aus der Meßserie zu $\theta^{\prime}=24^{\circ}$ ermittelte sechs Zahlenwerte bei sechs verschiedenen Geschwindigkeiten für die effektive Spaltbreite für Trimere. Die beste Anpassung wird erreicht bei dem Wert $\langle r\rangle_{\mathrm{g}}=18,5 \AA$. Rechts: Chiquadrat-Funktion.

\begin{tabular}{|c|c|}
\hline $\begin{array}{c}\text { Meßserie zum } \\
\text { Einfallswinkel } \theta^{\prime}[\mathrm{deg}]\end{array}$ & $\begin{array}{c}\text { Helium-Trimer } \\
\text { Paarabstand }\langle r\rangle_{\mathrm{g}}[\AA]\end{array}$ \\
\hline 18 & $12,0+5 /-8$ \\
21 & $10,0+5 /-7$ \\
24 & $18,5 \pm 2,5$ \\
\hline \hline
\end{tabular}

Tabelle 4.3: Ergebnisse für den Paarabstand des Trimers. 
$\theta^{\prime}=18^{\circ}$ und $21^{\circ}$ wurden am selben Gitter durchgeführt, wenn auch wahrscheinlich an etwas unterschiedlichen Positionen, da das Transmissionsgitter zwischen den beiden Messungen entfernt und später wieder eingesetzt wurde. Sie deuten auf die Reproduzierbarkeit des Experiments im Rahmen der Fehlerangaben hin. Außerdem streuen ihre Werte für die effektiven Spaltbreiten weniger stark, wie in den Abbildungen 4.9-4.11 zu erkennen ist. Andererseits sind die ermittelten Fehlerangaben des Paarabstandes der Messung bei $24^{\circ}$, die an einem anderen Gitter durchgeführt wurde, geringer. Jedoch handelt es sich bei dieser Messung um die früheste bei nicht-senkrechtem Einfall. Für sie liegt auch keine Vergleichsmessung zu senkrechtem Einfall vor, wohingegen für die beiden späteren Messungen zu $\theta^{\prime}=18^{\circ}$ und $21^{\circ}$ ein deutlich höherer Justageaufwand betrieben wurde. Diese Gründe können dafür angeführt werden, den Messungen zu $\theta^{\prime}=18^{\circ}$ und $21^{\circ}$ ein höheres Gewicht beizumessen. Für eine verläßliche Aussage und insbesondere für eine genauere Fehlerabschätzung ist allerdings eine Wiederholung der Experimente unumgänglich.

\subsection{Dimer-Beugungsbilder}

Die ebenfalls im Experiment aufgenommenen Dimer-Beugungsbilder werden hier der Vollständigkeit halber ausgewertet. Dazu ist die Kenntnis der Formeln für die Intensität im Beugungsbild sowie die effektive Spaltbreite für Dimere notwendig. Für senkrechten Einfall sind diese bekannt [5, 40]. Die Herleitung für nicht-senkrechten Einfall erfolgt analog, wobei die Ergebnisse der Kapitel 2 und 3 zu berücksichtigen sind; an dieser Stelle wird deshalb auf eine ausführliche Darstellung verzichtet. Die Intensitätsverteilung im Dimer-Beugungsbild wird ebenfalls durch die kumulantenentwickelte Intensitätsformel (3.113) beschrieben. Die effektive Spaltbreite eines aus zwei ununterscheidbaren Teilchen bestehenden Dimers ist zu interpretieren als

$$
\begin{aligned}
& S_{\text {eff }, \gamma}\left(v^{\prime}, \overline{\theta^{\prime}}\right) \simeq S_{0}-\frac{\left\langle\left|r_{\perp}\right|\right\rangle_{\gamma}}{\cos \phi^{\prime}}-\operatorname{Re} \int \mathrm{d}^{3} r\left|\phi_{\gamma}(\mathbf{r})\right|^{2} \\
& \quad \times\left\{\int_{0}^{\frac{S_{0}}{2}} \mathrm{~d} U_{2}^{\prime}\left[1-\tau_{u}^{\text {at }}\left(\frac{1}{2} \overline{\boldsymbol{P}}^{\prime} ; U_{2}^{\prime}\right) \tau_{u}^{\text {at }}\left(\frac{1}{2} \overline{\boldsymbol{P}}^{\prime} ; U_{2}^{\prime}-\frac{\left|r_{\perp}\right|}{\cos \phi^{\prime}}\right)\right]\right. \\
& \left.\quad+\int_{-\frac{S_{0}}{2}}^{0} \mathrm{~d} U_{2}^{\prime}\left[1-\tau_{u}^{\mathrm{at}}\left(\frac{1}{2} \overline{\boldsymbol{P}}^{\prime} ; U_{2}^{\prime}\right) \tau_{u}^{\mathrm{at}}\left(\frac{1}{2} \overline{\boldsymbol{P}}^{\prime} ; U_{2}^{\prime}+\frac{\left|r_{\perp}\right|}{\cos \phi^{\prime}}\right)\right]\right\}
\end{aligned}
$$

mit $v^{\prime}=\left|\overline{\boldsymbol{P}}^{\prime}\right| / M$, wobei $\mathbf{r}$ die Relativkoordinate und $\phi_{\gamma}(\mathbf{r})$ die Bindungswellenfunktion des freien Dimers im Ortsraum bezeichnen und $M=2 m$ die Dimermasse ist. Die folgende Diskussion beschränkt sich im Hinblick auf das Helium-Dimer auf Dimerzustände mit Drehimpulsquantenzahl $l=0$. Um den mittleren Paarabstand $\langle r\rangle_{\gamma}$ aus dem Experiment zu bestimmen, ist wiederum die Approximation des Integranden in Gleichung (4.4) notwendig. Im Unterschied zum Trimer gilt die Relation $\left\langle\left|r_{\perp}\right|\right\rangle_{\gamma}=\frac{1}{2}\langle r\rangle_{\gamma}$ hier exakt. So erhält man durch Vertauschung der Bildung des Erwartungswerts mit der Auswertung der Funktionen näherungsweise

$$
S_{\text {eff }, \gamma}\left(v^{\prime}, \overline{\theta^{\prime}}\right) \simeq S_{0}-\frac{\langle r\rangle_{\gamma}}{2 \cos \phi^{\prime}}
$$




$$
\begin{aligned}
-\operatorname{Re} & \left\{\int_{0}^{\frac{S_{0}}{2}} \mathrm{~d} U_{2}^{\prime}\left[1-\tau_{u}^{\mathrm{at}}\left(\frac{1}{2} \overline{\boldsymbol{P}}^{\prime} ; U_{2}^{\prime}\right) \tau_{u}^{\mathrm{at}}\left(\frac{1}{2} \overline{\boldsymbol{P}}^{\prime} ; U_{2}^{\prime}-\frac{\langle r\rangle_{\gamma}}{2 \cos \phi^{\prime}}\right)\right]\right. \\
& \left.+\int_{-\frac{S_{0}}{2}}^{0} \mathrm{~d} U_{2}^{\prime}\left[1-\tau_{u}^{\mathrm{at}}\left(\frac{1}{2} \overline{\boldsymbol{P}}^{\prime} ; U_{2}^{\prime}\right) \tau_{u}^{\mathrm{at}}\left(\frac{1}{2} \overline{\boldsymbol{P}}^{\prime} ; U_{2}^{\prime}+\frac{\langle r\rangle_{\gamma}}{2 \cos \phi^{\prime}}\right)\right]\right\} .
\end{aligned}
$$

Der Approximationsfehler $\delta_{\gamma}:=S_{\text {eff }, \gamma}(4.4)-S_{\text {eff }, \gamma}(4.5)$ muß mit Hilfe eines Modells für die Bindungswellenfunktion $\phi_{\gamma}(\mathbf{r})$ abgeschätzt werden. Für das Helium-Dimer, das nur im Grundzustand $\phi_{\mathrm{g}}(\mathbf{r})$ gebunden ist, ist er in den vorliegenden Experimenten bei $\theta^{\prime}=21^{\circ}$ und einer mittleren Geschwindigkeit von $v^{\prime}=500 \mathrm{~m} / \mathrm{s}$ von der Größenordnung $\delta_{\mathrm{g}} \approx-15 \AA$, wie eine numerische Rechnung basierend auf der DimerBindungwellenfunktion des TTY-Potentials [54] zeigt. Bei der Anpassung von Gleichung (4.5) an experimentelle Werte für die effektive Spaltbreite des Dimers führt dies zu einer Überschätzung des Paarabstandes $\langle r\rangle_{\mathrm{g}}$ um den Betrag $2 \cos \phi^{\prime} \delta_{\mathrm{g}} \approx 6 \AA$.
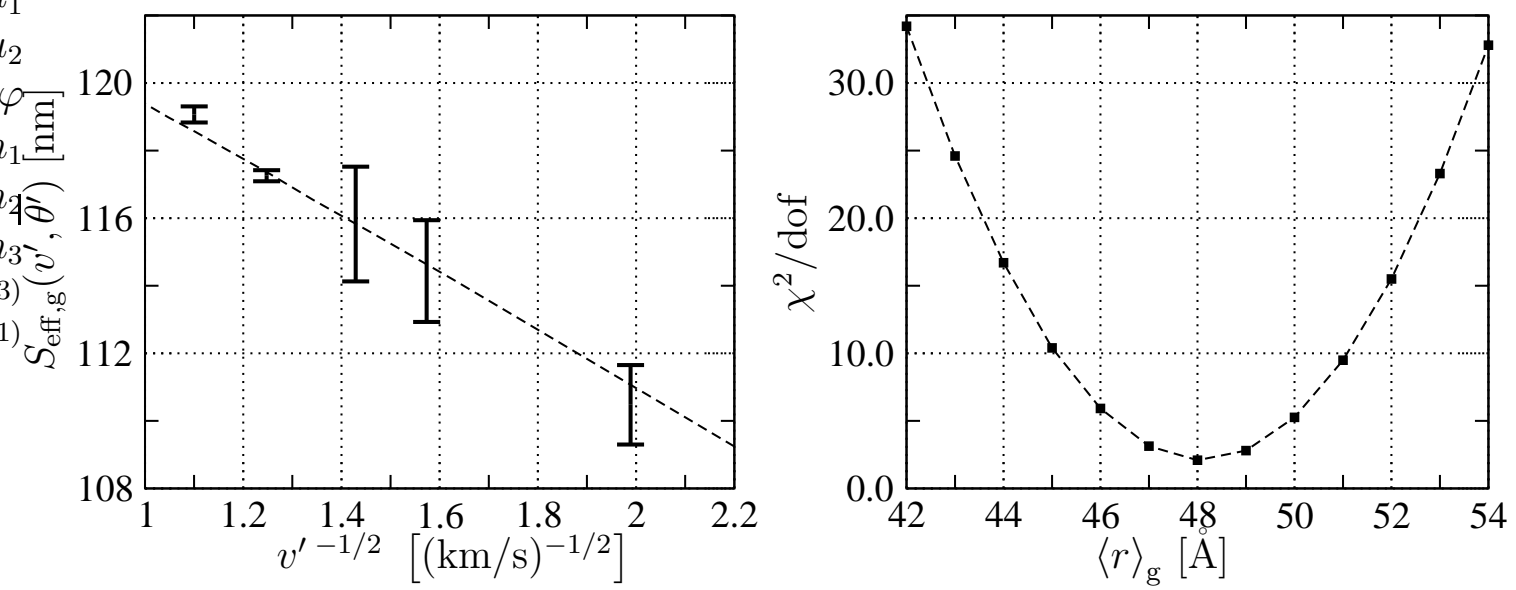

Abbildung 4.12: Links: Aus der Meßserie zu $\theta^{\prime}=21^{\circ}$ ermittelte fünf Zahlenwerte bei fünf verschiedenen Geschwindigkeiten für die effektive Spaltbreite für Dimere. Die beste Anpassung wird erreicht bei dem (unkorrigierten) Wert $\langle r\rangle_{\mathrm{g}}=48 \AA$. Rechts: ChiquadratFunktion.

In Abbildung 4.12 sind die aus einer Meßserie mit fünf Dimer-Beugungsbildern gewonnenen Werte für die effektive Spaltbreite dargestellt. Diese Meßserie ist Teil des Experiments zum Einfallswinkel $\theta^{\prime}=21^{\circ}$; die Gittergeometrie kann den Tabellen 4.1 und 4.2 entnommen werden. Die beste Anpassung erfolgt bei einem Paarabstand von $48 \AA$. Eine Fehlerangabe läßt sich analog zum Fall der Trimere gewinnen. Unter Berücksichtigung des Approximationsfehlers findet man als Ergebnis $\langle r\rangle_{\mathrm{g}}=48-6+$ $3 /-2 \AA$. Dies ist zu vergleichen mit dem in Ref. [5] publizierten Wert $\langle r\rangle_{\mathrm{g}}=52 \pm 4 \AA$.

Die als Teil der Experimente bei den Einfallswinkeln $\theta^{\prime}=18^{\circ}$ und $\theta^{\prime}=24^{\circ}$ aufgenommenen Dimer-Meßserien können nicht ausgewertet werden, da ihre in Abbildung 4.13 dargestellten effektiven Spaltbreiten nicht mit der Theorie verträglich sind. Es ist anzunehmen, daß bei diesen Meßserien nicht nachvollziehbare Störungen aufgetreten sind. 

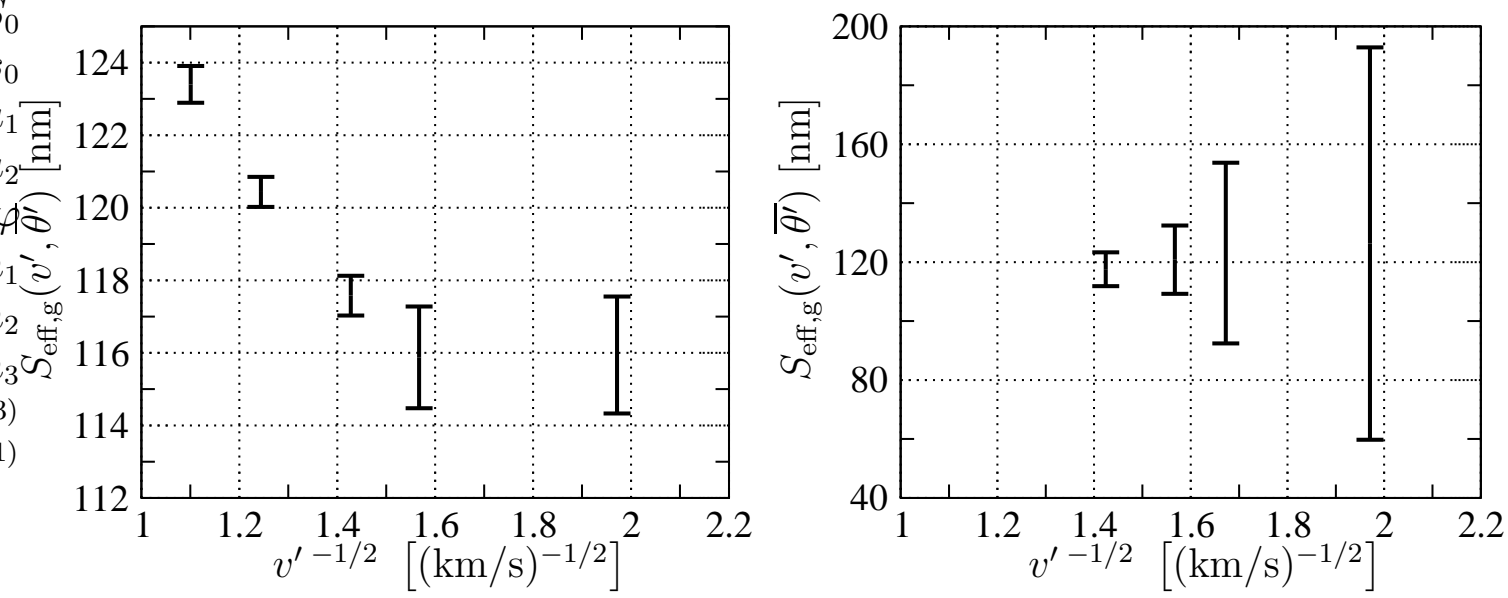

Abbildung 4.13: Aus den Meßserien zu $\theta^{\prime}=18^{\circ}$ (links) und $\theta^{\prime}=24^{\circ}$ (rechts) ermittelte Zahlenwerte für die effektive Spaltbreite für Dimere. Die Meßserien können nicht ausgewertet werden.

\subsubsection{Reduktion des Approximationsfehlers}

In der theoretischen Literatur zum Helium-Helium-Paarpotential besteht Interesse am genauen Wert des Dimer-Paarabstandes. Aktuelle theoretische Potentialmodelle stimmen in ihren Vorhersagen zwar qualitativ überein, unterscheiden sich jedoch bei der Bindungsenergie noch um bis zu 25\% sowie beim Paarabstand und der HeliumHelium-Streulänge um 10\% [54, 64, 65, 66]. Der systematische Approximationsfehler $\delta_{\mathrm{g}}\left(v^{\prime}, \theta^{\prime}\right)$ bewirkte aber im letzten Abschnitt eine Überschätzung des Paarabstandes in derselben Größenordnung. Für die Verbesserung der Genauigkeit der Auswertung ist daher zu erwägen, den Einfluß des Approximationsfehlers durch Hinzunahme verschiedener Potentialmodelle zu reduzieren. Zu diesem Zweck wird $\delta_{\mathrm{g}}\left(v^{\prime}, \theta^{\prime}\right)$ unter Verwendung der von diesen Potentialen getragenen Bindungswellenfunktionen im voraus tabelliert und in die Auswertung einbezogen. Falls das anschließende Resultat für den Paarabstand (weitesgehend) unabhängig vom verwendeten Potentialmodell ist, so ist eine glaubwürdige Verbesserung erreicht worden.

Um die schwache Abhängigkeit vom Potentialmodell zu überprüfen, wurden zwei aus der Literatur bekannte Potentiale herangezogen. Sie unterscheiden sich deutlich in ihren Vorhersagen für das Helium-Dimer: das ältere TTY-Potential [54] trägt beispielsweise eine Bindungswellenfunktion bei der Energie $E_{g}=-0,115 \mu \mathrm{eV}$ mit dem Paarabstand $\langle r\rangle_{\mathrm{g}}=51,6 \AA$, während das jüngere Potential von Gdanitz [66] $\mathrm{zu}\langle r\rangle_{\mathrm{g}}=46,4 \AA$ bei $E_{g}=-0,144 \mu \mathrm{eV}$ führt. Aus beiden Potentialen wurden die Bindungswellenfunktionen und anschließend $\delta_{\mathrm{g}}\left(v^{\prime}, \theta^{\prime}\right)$ berechnet. In der Tabelle 4.4 sind die numerischen Ergebnisse für die experimentellen Parameter aus den Tabellen 4.1/4.2 zusammengefaßt.

Anstelle von Gleichung (4.5) wird nun der auf die folgende Weise umformulierte Ausdruck zur Anpassung der effektiven Spaltbreiten verwendet:

$$
S_{\mathrm{eff}, \mathrm{g}}\left(v^{\prime}, \overline{\theta^{\prime}}\right) \simeq S_{0}-\frac{\langle r\rangle_{\mathrm{g}}}{2 \cos \phi^{\prime}}+\delta_{\mathrm{g}}\left(v^{\prime}, \theta^{\prime}\right)
$$




\begin{tabular}{|c|cc|}
\hline$v^{\prime}[\mathrm{m} / \mathrm{s}]$ & \multicolumn{2}{|c|}{$\delta_{\mathrm{g}}\left(v^{\prime}, \theta^{\prime}\right)[\mathrm{A}]$} \\
& TTY & Gdanitz \\
\hline 826,1 & $-12,6$ & $-10,2$ \\
642,3 & $-13,8$ & $-11,3$ \\
489,6 & $-15,6$ & $-12,8$ \\
403,9 & $-16,6$ & $-13,8$ \\
252,8 & $-20,2$ & $-17,0$ \\
\hline \hline
\end{tabular}

Tabelle 4.4: Der Approximationsfehler $\delta_{\mathrm{g}}\left(v^{\prime}, \theta^{\prime}\right)$ bei $\theta^{\prime}=21^{\circ}$ und den fünf experimentellen Geschwindigkeiten aus Abb. 4.12, berechnet mit Hilfe der Bindungswellenfunktion des TTY-Potentials [54] und des Potentials von Gdanitz [66].

$$
\begin{aligned}
-\operatorname{Re} & \left\{\int_{0}^{\frac{S_{0}}{2}} \mathrm{~d} U_{2}^{\prime}\left[1-\tau_{u}^{\mathrm{at}}\left(\frac{1}{2} \overline{\boldsymbol{P}}^{\prime} ; U_{2}^{\prime}\right) \tau_{u}^{\mathrm{at}}\left(\frac{1}{2} \overline{\boldsymbol{P}}^{\prime} ; U_{2}^{\prime}-\frac{\langle r\rangle_{\mathrm{g}}}{2 \cos \phi^{\prime}}\right)\right]\right. \\
& \left.+\int_{-\frac{S_{0}}{2}}^{0} \mathrm{~d} U_{2}^{\prime}\left[1-\tau_{u}^{\mathrm{at}}\left(\frac{1}{2} \overline{\boldsymbol{P}}^{\prime} ; U_{2}^{\prime}\right) \tau_{u}^{\mathrm{at}}\left(\frac{1}{2} \overline{\boldsymbol{P}}^{\prime} ; U_{2}^{\prime}+\frac{\langle r\rangle_{\mathrm{g}}}{2 \cos \phi^{\prime}}\right)\right]\right\} .
\end{aligned}
$$

Für $\delta_{\mathrm{g}}\left(v^{\prime}, \theta^{\prime}\right)$ sind die Zahlenwerte aus Tabelle 4.4 einzusetzen. Die Neuauswertung der effektiven Spaltbreiten mittels Gleichung (4.4') führt daraufhin auf die Ergebnisse $\langle r\rangle_{\mathrm{g}}=42,6+2,6 /-2,4 \AA$ (TTY-Potential) und $\langle r\rangle_{\mathrm{g}}=43,6+2,6 /-2,3 \AA$ (Potential von Gdanitz). Die Modellabhängigkeit beträgt hier also nur circa $2 \%$ des Paarabstandes und ist kleiner als die experimentellen Fehlergrenzen. Als „korrigiertes“ Ergebnis wird für den Paarabstand des Helium-Dimers der Mittelwert beider Auswertungen angegeben, also $\langle r\rangle_{\mathrm{g}}=43+3 /-3 \AA$. In Tabelle 4.5 sind die Ergebnisse noch einmal

\begin{tabular}{|c|c|c|c|}
\hline Helium-Dimer & $\langle r\rangle_{\mathrm{g}}[\AA]$ & $E_{\mathrm{g}}[\mu \mathrm{eV}]$ & $a_{0}[\stackrel{\mathrm{A}}{]}]$ \\
\hline diese Arbeit (korrigiert) & $43+3 /-3$ & $0,14+0,03 /-0,02$ & $86+6 /-15$ \\
\hline diese Arbeit (unkorrigiert) & $48+3 /-8$ & $0,11+0,04 /-0,01$ & $96+6 /-26$ \\
\hline Ref. [5] (unkorrigiert) & $52+4 /-4$ & $0,10+0,03 /-0,02$ & $104+8 /-18$ \\
\hline
\end{tabular}
zusammengefaßt.

Tabelle 4.5: Auswertung der Meßserie für das Helium-Dimer des Experiments bei $\theta^{\prime}=21^{\circ}$. Das „unkorrigierte“ Ergebnis ist zu vergleichen mit dem Wert aus Ref. [5]. Ebenfalls angegeben sind die daraus berechnete Bindungsenergie $\left|E_{g}\right| \simeq \hbar^{2} / 4 m\langle r\rangle_{\mathrm{g}}^{2}$ und die Streulänge der Helium-Helium-Streuung $a_{0} \simeq 2\langle r\rangle_{\mathrm{g}}$ (Ref. [67, Kap. II] und [68]). Diese Formeln unterschätzen die Bindungsenergie und überschätzen die Streulänge um circa 10\% [5]. Die Fehlerangaben wurden entsprechend vergrößert. 


\section{Kapitel 5}

\section{Inelastische Beugung und Spektroskopie}

Die vorigen Kapitel behandelten schwerpunktmäßig die Berechnung und die physikalische Interpretation von elastischer Beugung sowie die Auswertung entsprechender experimenteller Daten. Der streutheoretische Formalismus aus Kapitel 3 enthält jedoch auch die Anregung innerer Freiheitsgrade der gebeugten Cluster. Eine solche Anregung führt zu inelastischer Beugung, die sich auf charakteristische Weise durch zusätzliche Beugungsmaxima im Beugungsbild äußert. Bislang sind noch keine Experimente bekannt, die sich die Eigenschaften inelastischer Beugung von van der Waals-Clustern zunutze machen. Wie dieses Kapitel zeigt, ist die Winkelauflösung unproblematisch - die experimentelle Herausforderung besteht in der geringen Anregungswahrscheinlichkeit.

Das zugrundeliegende Prinzip wurde in experimentellen Arbeiten von Boustimi et al anhand der inelastischen Beugung von metastabilen Argon-Atomen [69] und Stickstoff-Dimeren [70] an einem Transmissionsgitter unter Verwendung eines Feinstruktur- beziehungsweise eines Vibrationsübergangs demonstriert. Allerdings konnten dort aufgrund der zu geringen räumlichen Kohärenz des Atomstrahls noch keine diskreten Beugungsmaxima aufgelöst werden; das Transmissionsgitter diente lediglich zur Vervielfachung der Beugungsamplitude des Einzelspalts. In einer weiteren Arbeit derselben Gruppe wurde die Anregung eines Feinstrukturübergangs von metastabilem Argon an einem Kupferspalt verwendet [71].

Auf theoretischer Seite wurde von Hegerfeldt und Köhler im Rahmen der Beugung von Helium-Trimeren bereits die Erzeugung des angeregten Trimer-Zustandes aus dem Grundzustand durch inelastische Beugung untersucht [63]. Da die Existenz des angeregten Zustandes allerdings bislang experimentell nicht erwiesen ist, wird in diesem Kapitel ein realistisches Beugungsexperiment unter Verwendung der gut bekannten und mit bestehenden Strahlquellen einfach handhabbaren Wasserstoff-Dimere $\left(\mathrm{D}_{2}\right)_{2}$ und $\mathrm{H}_{2} \mathrm{D}_{2}$ vorgeschlagen und quantitativ analysiert. Es zeigt sich dabei, daß es prinzipiell möglich ist, das Energie-Spektrum solcher und anderer van der WaalsCluster durch kohärente, inelastische Beugung experimentell zu ermitteln. Die inelastische Beugung stellt somit eine neue Möglichkeit der Cluster-Spektroskopie dar. Da sie nicht auf elektromagnetischer Ankopplung an die inneren Freiheitsgrade des 
Clusters beruht, komplementiert sie übliche spektroskopische Techniken wie etwa die Infrarot-Absorptionsspektroskopie [41]. Darüberhinaus ermöglicht sie beispielsweise die Erzeugung eines reinen Teilstrahls von Clustern mit vorgegebenen inneren Zuständen, indem alle Beugungsordnungen bis auf die gewünschte ausgefiltert werden.

Das Thema des vorliegenden Kapitels wurde in Ref. [72] noch mit einer vereinfachten Theorie der Beugung für nicht-senkrechten Einfall behandelt. Diese vereinfachte Theorie hat den Vorteil, daß sie in ihrem Gültigkeitsbereich bei hohen Strahlgeschwindigkeiten in beiden Regimen $\theta^{\prime}<\beta$ und $\beta<\theta^{\prime}$ anwendbar ist und somit das stetige Vergrößern des Einfallswinkels ausgehend von $\theta^{\prime}=0$ über $\beta$ hinaus mit demselben Formalismus zu beschreiben vermag. Das vorliegende Kapitel verwendet dagegen die in Kapitel 2 entwickelte Theorie der Beugung am Transmissionsgitter, die die Intensitäten der Beugungsmaxima für $\beta<\theta^{\prime}$ quantitativ genauer beschreibt.

\subsection{Inelastische Dimerbeugung}

In Abschnitt 3.4.1 wurden im Rahmen der Trimerbeugung Formeln für die Beugungswinkel (3.102) sowie die Intensitäten (3.104) eines kollimierten Teilchenstrahls abgeleitet. Es wurde dort bereits angemerkt, daß diese Formeln prinzipiell für beliebige, schwach gebundene Cluster gültig sind. In diesem Kapitel können sie daher ohne die Notwendigkeit einer erneuten Herleitung übernommen werden.

\subsubsection{Dimerbeugung am Gitter bei nicht-senkrechtem Ein- fall}

Ein Dimer wird im folgenden modelliert durch zwei Teilchen der Massen $m_{1}$ und $m_{2}$, die sich an den Orten $\mathbf{r}_{1}$ beziehungsweise $\mathbf{r}_{2}$ befinden. Ihre Wechselwirkung wird durch ein zentralsymmetrisches Potential $v(|\mathbf{r}|)$ beschrieben, wobei $\mathbf{r}:=\mathbf{r}_{1}-\mathbf{r}_{2}$ die Relativkoordinate bezeichnet. Weiterhin werden die Gesamtmasse $M:=m_{1}+m_{2}$ und die reduzierte Masse $\mu:=m_{1} m_{2} /\left(m_{1}+m_{2}\right)$ sowie die Schwerpunktkoordinate $\mathbf{R}:=\left(m_{1} \mathbf{r}_{1}+m_{2} \mathbf{r}_{2}\right) / M$ eingeführt. Die Teilchen dürfen einen Spin tragen. Die Wechselwirkung des Teilchens $i$ mit dem Transmissionsgitter wird durch das Potential $W_{i}\left(\mathbf{x}_{i}\right)$ repräsentiert. Der vollständige Hamilton-Operator für die Dimerbeugung lautet

$$
H=\frac{\hat{\mathbf{P}}^{2}}{2 M}+\frac{\hat{\mathbf{p}}^{2}}{2 \mu}+W_{1}\left(\hat{\mathbf{r}}_{1}\right)+W_{2}\left(\hat{\mathbf{r}}_{2}\right)+v(|\hat{\mathbf{r}}|),
$$

wobei wie in Abschnitt 3.2 eine etwaige Mehrteilchenwechselwirkung vernachlässigt wird. $\hat{\mathbf{P}}$ und $\hat{\mathbf{p}}$ bezeichnen den Schwerpunkt- und den Relativimpuls-Operator.

Für senkrechten Einfall auf das Transmissionsgitter ist die Dimer-Übergangsamplitude aus früheren Arbeiten bekannt [3, 72]. Für nicht-senkrechten Einfall kann sie auf analoge Weise mit Hilfe des in den Kapiteln 2 und 3 vorgestellten Zugangs berechnet werden. Auf eine Herleitung wird an dieser Stelle verzichtet. Unter Verwendung der in den vorigen Kapiteln eingeführten Notation findet man für die DimerÜbergangsamplitude den Ausdruck (vgl. Gl. 3.99)

$$
t^{(2)}\left(\boldsymbol{P}, \phi_{\gamma} ; \boldsymbol{P}^{\prime}, \phi_{\gamma^{\prime}}\right) \simeq
$$




$$
-\frac{\mathrm{i}}{2} \frac{\Pi_{1}+\Pi_{1}^{\prime}}{(2 \pi)^{2} M \hbar}\left\{2 \pi \hbar \frac{P_{1}}{\Pi_{1}} \delta\left(\Delta P_{2}\right) \delta_{\gamma \gamma^{\prime}}-H_{N}\left(\Delta P_{2}\right) a_{u, \gamma \gamma^{\prime}}^{\operatorname{dim}}\left(\boldsymbol{P}^{\prime} ; \Delta \Pi_{2}\right)\right\} .
$$

Die Spaltamplitude des Dimers lautet darin (vgl. Gl. 3.98)

$$
a_{u, \gamma \gamma^{\prime}}^{\operatorname{dim}}\left(\boldsymbol{P}^{\prime} ; \Delta \Pi_{2}\right):=\int_{-\frac{S_{0}}{2}}^{\frac{S_{0}}{2}} \mathrm{~d} U_{2} \exp \left(-\mathrm{i} \Delta \Pi_{2} U_{2} / \hbar\right) \tau_{u, \gamma \gamma^{\prime}}^{\operatorname{dim}}\left(\boldsymbol{P}^{\prime} ; U_{2}\right),
$$

und die Dimer-Transmissionsfunktion ist definiert durch (vgl. Gl. 3.96)

$$
\begin{aligned}
\tau_{u, \gamma \gamma^{\prime}}^{\operatorname{dim}}\left(\boldsymbol{P}^{\prime} ; U_{2}\right) & :=\int \mathrm{d}^{3} r \phi_{\gamma}^{*}(\mathbf{r}) \phi_{\gamma^{\prime}}(\mathbf{r}) \\
& \times \tau_{1, u}^{\mathrm{at}}\left(\frac{m_{1}}{M} \boldsymbol{P}^{\prime} ; U_{2}+\frac{m_{2}}{M} \frac{r_{\perp}}{\cos \phi^{\prime}}\right) \tau_{2, u}^{\mathrm{at}}\left(\frac{m_{2}}{M} \boldsymbol{P}^{\prime} ; U_{2}-\frac{m_{1}}{M} \frac{r_{\perp}}{\cos \phi^{\prime}}\right) .
\end{aligned}
$$

\subsubsection{Symmetrie und Auswahlregel der Dimerbeugung}

Bindungswellenfunktionen des Dimers sind die quadrat-integrablen Lösungen des Relativanteils der freien Schrödinger-Gleichung zu negativen Bindungsenergien $E_{\gamma}$.

$$
\left[\frac{\hat{\mathbf{p}}^{2}}{2 \mu}+v(|\mathbf{r}|)\right] \phi_{\gamma}(\mathbf{r})=E_{\gamma} \phi_{\gamma}(\mathbf{r})
$$

Wegen der Zentralsymmetrie des Paarpotentials können sie zu festen Drehimpulsquantenzahlen $l$ und $m$ gewählt werden. Unter Verwendung der Definitionen $r:=|\mathbf{r}|$ und $\widehat{\mathbf{r}}:=\mathbf{r} / r$ lassen sie sich wie üblich in ein Produkt $\phi_{h l m}(\mathbf{r}) \equiv R_{h l}(r) Y_{l}^{m}(\widehat{\mathbf{r}})$ zerlegen, wobei $R_{h l}(r)$ eine reell wählbare Lösung des Radialanteils der SchrödingerGleichung (5.5) ist ${ }^{1}$ und $Y_{l}^{m}(\widehat{\mathbf{r}})$ eine Kugelfächenfunktion bezeichnet.

Da der winkelabhängige Teil der Bindungswellenfunktionen explizit bekannt ist, kann eine Integration in der Dimer-Transmissionsfunktion (5.4) ausgeführt werden. Dazu wird die Koordinatenrichtung $x_{\perp}$ als Quantisierungsachse der Kugelfächenfunktionen gewählt und zu Kugelkoordinaten $(r, \vartheta, \varphi)$ übergewechselt. Es gilt also $r_{\perp}=r \cos \vartheta$. Die Integration über den Azimutalwinkel $\varphi$ führt im wesentlichen auf ein Produkt zweier assoziierter Legendre-Polynome, das in der folgenden Form geschrieben wird:

$$
\int_{0}^{2 \pi} \mathrm{d} \varphi\left(Y_{l}^{m}\right)^{*}(\widehat{\mathbf{r}}) Y_{l^{\prime}}^{m^{\prime}}(\widehat{\mathbf{r}})=\delta_{m m^{\prime}} \sqrt{2 l+1} \sqrt{2 l^{\prime}+1} \Pi_{l l^{\prime}}^{m^{\prime}}(\cos \vartheta) .
$$

Darin wurde die Funktion $\Pi_{l l^{\prime}}^{m^{\prime}}(\cos \vartheta)$ definiert als

$$
\Pi_{l l^{\prime}}^{m^{\prime}}(\alpha):=\frac{1}{2} \sqrt{\frac{\left(l-\left|m^{\prime}\right|\right) !}{\left(l+\left|m^{\prime}\right|\right) !} \frac{\left(l^{\prime}-\left|m^{\prime}\right|\right) !}{\left(l^{\prime}+\left|m^{\prime}\right|\right) !}} P_{l}^{\left|m^{\prime}\right|}(\alpha) P_{l^{\prime}}^{\left|m^{\prime}\right|}(\alpha), \quad\left|m^{\prime}\right| \leq \min \left(l, l^{\prime}\right) .
$$

\footnotetext{
${ }^{1}$ Um eine Verwechslung mit der Beugungsordnung $n$ zu vermeiden, wird die Hauptquantenzahl hier mit $h$ bezeichnet.
} 
Mit der Substitution $\alpha:=\cos \vartheta$ lautet die Dimer-Transmissionsfunktion für die inelastische Beugung des Zustandes $\gamma^{\prime} \widehat{=} h^{\prime} l^{\prime} m^{\prime}$ in den Zustand $\gamma \widehat{=} h l m$ also

$$
\begin{aligned}
\tau_{u, h l m h^{\prime} l^{\prime} m^{\prime}}^{\operatorname{dim}}\left(\boldsymbol{P}^{\prime} ; U_{2}\right) & =\delta_{m m^{\prime}} \sqrt{2 l+1} \sqrt{2 l^{\prime}+1} \int_{0}^{\infty} \mathrm{d} r r^{2} R_{h l}(r) R_{h^{\prime} l^{\prime}}(r) \int_{-1}^{1} \mathrm{~d} \alpha \Pi_{l l^{\prime}}^{m^{\prime}}(\alpha) \\
& \times \tau_{1, u}^{\mathrm{at}}\left(\frac{m_{1}}{M} \boldsymbol{P}^{\prime} ; U_{2}+\frac{m_{2}}{M} \frac{r \alpha}{\cos \phi^{\prime}}\right) \tau_{2, u}^{\mathrm{at}}\left(\frac{m_{2}}{M} \boldsymbol{P}^{\prime} ; U_{2}-\frac{m_{1}}{M} \frac{r \alpha}{\cos \phi^{\prime}}\right) .
\end{aligned}
$$

Im Fall zweier ununterscheidbarer Teilchen der Masse $m=M / 2$ ist der vollständige Hamilton-Operator (5.1) invariant unter Teilchenaustausch. Seine Lösungen können daher zu fester Parität unter Inversion der Relativkoordinate $\mathbf{r}$ gewählt werden. Mit anderen Worten bedeutet dies, daß die Parität während der Beugung erhalten bleibt. Ein asymptotisch mit fester Drehimpulsquantenzahl $l^{\prime}$ einlaufendes Dimer hat Parität $(-1)^{l^{\prime}}$; entsprechend ist die Parität eines asymptotisch mit Drehimpulsquantenzahl $l$ auslaufenden Dimers $(-1)^{l}$. Somit läßt sich bei ununterscheidbaren Teilchen die folgende Auswahlregel aufstellen:

$$
l^{\prime}+l=\text { gerade. }
$$

Konkret wird sie durch die Parität $(-1)^{l+l^{\prime}}$ der Funktion $\Pi_{l l^{\prime}}^{m^{\prime}}(\alpha)$ widergespiegelt, die bei Verletzung der Auswahlregel zu einem Verschwinden der Dimer-Transmissionsfunktion (5.7) führt. Bei unterscheidbaren Teilchen existiert keine derartige Auswahlregel.

Laut Gleichung (3.102) werden die Winkel, unter denen die inelastischen Beugungsmaxima auftreten, durch den Energieübertrag bei der An- oder Abregung bestimmt. Wegen der Zentralsymmetrie der Paarwechselwirkung sind die Bindungsenergien aber bezüglich $m^{\prime}$ entartet; einlaufende Zustände gleicher Haupt- und Drehimpulsquantenzahlen werden deshalb in gleiche Winkel gebeugt:

$$
\sin \left(\theta_{n}^{h l h^{\prime} l^{\prime}}\right)=\left(1-\frac{E_{h l}-E_{h^{\prime} l^{\prime}}}{\left|\overline{\boldsymbol{P}}^{\prime}\right|^{2} / 2 M}\right)^{-\frac{1}{2}}\left[\sin \overline{\theta^{\prime}}+\frac{n 2 \pi \hbar}{\left|\overline{\boldsymbol{P}}^{\prime}\right| d}\right] .
$$

Im Experiment kann folglich entsprechend Gleichung (3.46) nur die inkohärente Überlagerung der Teilbeugungsbilder zu den möglichen Werten von $m^{\prime}$ gemessen werden,

$$
I_{n}^{h l h^{\prime} l^{\prime}} \propto\left(\frac{\Pi_{1}+\bar{\Pi}_{1}^{\prime}}{2\left|\overline{\boldsymbol{P}}^{\prime}\right| d \cos \overline{\theta^{\prime}}}\right)^{2} \sum_{m^{\prime}} p_{h^{\prime} l^{\prime} m^{\prime}}\left|a_{u, h l m^{\prime} h^{\prime} l^{\prime} m^{\prime}}^{\operatorname{dim}}\left(\overline{\boldsymbol{P}}^{\prime} ; \Delta \Pi_{2}\right)\right|^{2},
$$

wobei $p_{h^{\prime} l^{\prime} m^{\prime}}$ die relative Besetzungszahl des Zustandes $\phi_{h^{\prime} l^{\prime} m^{\prime}}$ im einlaufenden Strahl angibt. Durch den Faktor $\delta_{m m^{\prime}}$ in der Dimer-Transmissionsfunktion (5.7) ist die Summation in der Intensitätsformel (5.10) auf den Wertebereich $\left|m^{\prime}\right|<\min \left(l, l^{\prime}\right)$ beschränkt. Die Komponenten $\bar{\Pi}_{1}^{\prime}, \bar{\Pi}_{2}^{\prime}$ sind beim mittleren einlaufenden Schwerpunktimpuls $\overline{\boldsymbol{P}}^{\prime}$ auszuwerten, die Komponenten $\Pi_{1}, \Pi_{2}$ beim durch den Winkel (5.9) und die Energieerhaltung festgelegten auslaufenden Impuls $\boldsymbol{P}$ des betrachteten Übergangs. Das gesamte Beugungsbild des Dimer-Strahls schließlich setzt sich zusammen aus den Beugungsmaxima (5.10) aller durch die Auswahlregel erlaubten Übergänge. 


\subsection{Beugung von Wasserstoff-Dimeren}

Die Ergebnisse des letzten Abschnitts werfen die Frage auf, ob ein Experiment zur inelastischen Beugung von van der Waals-Dimeren mit einer Molekularstrahlapparatur erfolgreich durchführbar ist. Energieüberträge, die typischerweise im Bereich einiger hundert $\mu \mathrm{eV}$ liegen, entsprechen laut Gleichung (5.9) einer Winkelverschiebung der inelastischen Beugungsmaxima um einige Milliradian relativ zu den elastischen Beugungsmaxima. Diese Verschiebung ist somit von der gleichen Größenordnung wie die Abstände der elastischen Beugungsmaxima, und die Winkelauflösung stellt folglich kein technisches Problem dar. Die Übergangswahrscheinlichkeit hingegen entscheidet, ob inelastische Beugungsordnungen intensiv genug sind, um überhaupt beobachtet werden zu können. Sie hängt von den Dimeren und von der genauen Gittergeometrie ab und ist allgemein nur schwer abzuschätzen.

Als Modellsystem werden im folgenden die beiden Wasserstoff-Dimere $\left(\mathrm{D}_{2}\right)_{2}$ und $\mathrm{H}_{2} \mathrm{D}_{2}$ und die Gittergeometrie aus Kapitel 4 betrachtet. Gemeinsam mit $\left(\mathrm{H}_{2}\right)_{2}$ wurden diese Dimere sowohl theoretisch als auch experimentell ausführlich untersucht, nachdem in astrophysikalischen Planetenspektren Hinweise auf die Existenz von $\left(\mathrm{H}_{2}\right)_{2}$ gefunden worden waren [41, 73, 74, 75, 76]. Ihre Bindungspotentiale sind in sehr guter Approximation zentralsymmetrisch. Daß sie darüberhinaus nur wenige Bindungszustände besitzen, erleichtert die folgende Diskussion und das mögliche Experiment.

\subsubsection{Die freien Wasserstoff-Dimere $\left(\mathrm{o}-\mathrm{D}_{2}\right)_{2}$ und $\mathrm{p}-\mathrm{H}_{2}-\mathrm{o}-\mathrm{D}_{2}$}

Der Kern des Wasserstoffatoms H, das Proton, besitzt einen Spin von $\frac{1}{2}$ (in der Einheit $\hbar$ ); im Molekül $\mathrm{H}_{2}$ koppeln die Spins beider Kerne zu $I=0$ oder $I=1$. Im ersteren Fall ist die Spinwellenfunktion antisymmetrisch (Singulett) unter Teilchenaustausch, im letzteren Fall symmetrisch (Triplett). Da das Proton ein Fermion ist, kann der Raumanteil der Kernwellenfunktion des $\mathrm{H}_{2}$ beim Singulett nur gerade und beim Triplett nur ungerade Bahndrehimpulse $j$ annehmen. Man unterscheidet diese als para $\left(\mathrm{p}-\mathrm{H}_{2}\right)$ und ortho $\left(\mathrm{o}-\mathrm{H}_{2}\right)$ Modifikationen. Im freien $\mathrm{H}_{2}$-Gas stellt sich im Mittel eine Mischung von $75 \%$ o- $\mathrm{H}_{2}$ und $25 \%$ p- $\mathrm{H}_{2}$ ein.

Der Kern des Deuteriums D, das Deuteron, besitzt einen Spin von 1 [67]; entsprechend koppeln die Kernspins im Molekül $\mathrm{D}_{2}$ zum Gesamtspin $I=0,1$ oder 2. Wie eine kurze Rechnung zeigt, ist die Spinwellenfunktion des Tripletts mit $I=1 \mathrm{im} \mathrm{Ge-}$ gensatz zu $\mathrm{H}_{2}$ antisymmetrisch, wohingegen die Gesamtspins $I=0$ (Singulett) und $I=2$ (Quintett) zu symmetrischen Spinwellenfunktionen gehören. Da das Deuteron ein Boson ist, können die Triplettzustände nur antisymmetrische Bahndrehimpulse $j$ annehmen, die Singulett- und Quintettzustände jeweils nur symmetrische. Analog zum $\mathrm{H}_{2}$-Molekül unterscheidet man auch hier die Modifikationen $\mathrm{p}-\mathrm{D}_{2}$ (Triplett) und o- $\mathrm{D}_{2}$ (Singulett und Quintett); $67 \%$ der Moleküle liegen im freien $\mathrm{D}_{2}$-Gas als o- $\mathrm{D}_{2}$ vor.

Zur Vereinfachung der Diskussion wird im folgenden angenommen, daß durch geeignete experimentelle Maßnahmen, beispielsweise indem das Gas von Wasserstoffund Deuteriummolekülen vor der Düsenexpansion durch einen katalytischen Konver- 
ter geleitet wird [77], nur jene Modifikationen der Moleküle mit geraden Bahndrehimpulsen $j$ im Molekularstrahl vorhanden sind, also $\mathrm{p}-\mathrm{H}_{2}$ und o- $\mathrm{D}_{2}$. Darüberhinaus wird angenommen, daß der Großteil der Moleküle sich im Rotationsgrundzustand $j=0$ befindet; dies ist aufgrund der relativ geringen Trägheitsmomente der Moleküle $\mathrm{H}_{2}$ und $\mathrm{D}_{2}$ und der daraus folgenden hohen Anregungsenergien für den ersten Rotationsübergang $j=0 \rightarrow 2$ von $22 \mathrm{meV}$ im o- $\mathrm{D}_{2}$ beziehungsweise $44 \mathrm{meV}$ im p- $\mathrm{H}_{2}$ [78] bereits bei Düsentemperaturen von $T \lesssim 40 \mathrm{~K}$ entsprechend einer Strahlgeschwindigkeit von $v^{\prime} \lesssim 650 \mathrm{~ms}^{-1}$ der Fall [78, 79]. Nach diesen Voraussetzungen sollten die Moleküle näherungsweise als sphärisch symmetrisch betrachtet werden können. Daher sollte auch die Paarwechselwirkung zwischen zwei Molekülen näherungsweise zentralsymmetrisch sein. Diese Überlegungen und die Tatsache, daß die Dimere $\left(\mathrm{o}-\mathrm{D}_{2}\right)_{2}$ und $\mathrm{p}-\mathrm{H}_{2}-\mathrm{O}-\mathrm{D}_{2}$ eine relativ große räumliche Ausdehnung gegenüber den kovalent gebundenen Molekülen $\mathrm{p}-\mathrm{H}_{2}$ und $\mathrm{o}-\mathrm{D}_{2}$ haben, rechtfertigen im folgenden die Behandlung der Moleküle als Punktteilchen ohne innere Freiheitsgrade.

Für die explizite Berechnung des Beugungsbildes von $\left(\mathrm{o}-\mathrm{D}_{2}\right)_{2}$ und $\mathrm{p}-\mathrm{H}_{2}-\mathrm{O}-\mathrm{D}_{2}$ ist die Kenntnis der Dimer-Bindungswellenfunktionen nötig. $\mathrm{Zu}$ diesem Zweck wurde ein semi-empirisches Paarpotential von Buck et al [80] gewählt ${ }^{2}$, das in analytischer Form vorliegt. Nur der zentralsymmetrische Anteil des Potentials wurde berücksichtigt. Er ist gegeben durch einen repulsiven Kern und eine exponentiell gedämpfte Dispersionsreihe,

$$
v(r)=A \exp \left[-\beta r-\gamma r^{2}\right]-\left[C_{6} r^{-6}+C_{8} r^{-8}+C_{10} r^{-10}\right] f(r),
$$

mit

$$
f(r):=\left\{\begin{array}{cl}
\exp \left[-\left(r_{1} / r-1\right)^{2}\right] & : r \leq r_{1} \\
1 & : r \geq r_{1}
\end{array}\right.
$$

wobei die Konstanten die Werte $A=101,40 \mathrm{eV}, \beta=2,77992 \AA^{-1}, \gamma=0,08005 \AA^{-2}$, $r_{1}=5,100 \AA, C_{6}=7,239 \mathrm{eV \AA}^{6}, C_{8}=35,91 \mathrm{eVA}^{8}, C_{10}=224,79 \mathrm{eV \AA}^{10}$ annehmen. Das Paarpotential repräsentiert die elektronische Wechselwirkung der Moleküle und ist deswegen in sehr guter Näherung unabhängig von den Wasserstoffisotopen; die Schrödinger-Gleichung (5.5) für das $\left(\mathrm{o}-\mathrm{D}_{2}\right)_{2}$ unterscheidet sich von der für das $\mathrm{p}-\mathrm{H}_{2}-$ $\mathrm{o}-\mathrm{D}_{2}$ im wesentlichen nur durch die reduzierte Masse $\mu$. Diese bewirkt allerdings eine deutliche Verschiebung der Bindungsenergien.

Die numerische Lösung des Radialanteils der Schrödinger-Gleichung mit dem Paarpotential (5.11) liefert vier Bindungszustände für $\left(\mathrm{o}-\mathrm{D}_{2}\right)_{2}$ und drei für $\mathrm{p}-\mathrm{H}_{2}-$ o- $\mathrm{D}_{2}$, die jeweils zur niedrigsten Hauptquantenzahl $h=1$ gehören (in der Terminologie der Molekülphysik entspricht dies der niedrigsten Vibrationsanregung $\nu=0$ ). Die Hauptquantenzahl wird daher im folgenden nicht zur Kennzeichnung der DimerBindungswellenfunktionen benötigt und wird weggelassen. In Tabelle 5.1 sind die aus der numerischen Lösung der Schrödinger-Gleichung erhaltenen Dimer-Bindungsenergien $E_{l}$ aufgeführt. Sie stimmen für die Zwecke dieses Kapitels genügend gut mit Literaturdaten aus [74] überein, denen eine aufwendigere Berechnung zugrundeliegt. Abbildung 5.1 zeigt die Radialwellenfunktionen $R_{l}(r)$. Die Erwartungwerte des Paarabstandes liegen zwischen $\langle r\rangle=4,2 \ldots 4,6 \AA$ beim $\left(\mathrm{o}-\mathrm{D}_{2}\right)_{2}$ und $\langle r\rangle=4,5 \ldots 5,1 \AA$

\footnotetext{
${ }^{2}$ Für eine Übersicht zu Paarpotentialen für das Wasserstoff-Dimer siehe z. B. Ref. [81, 82].
} 


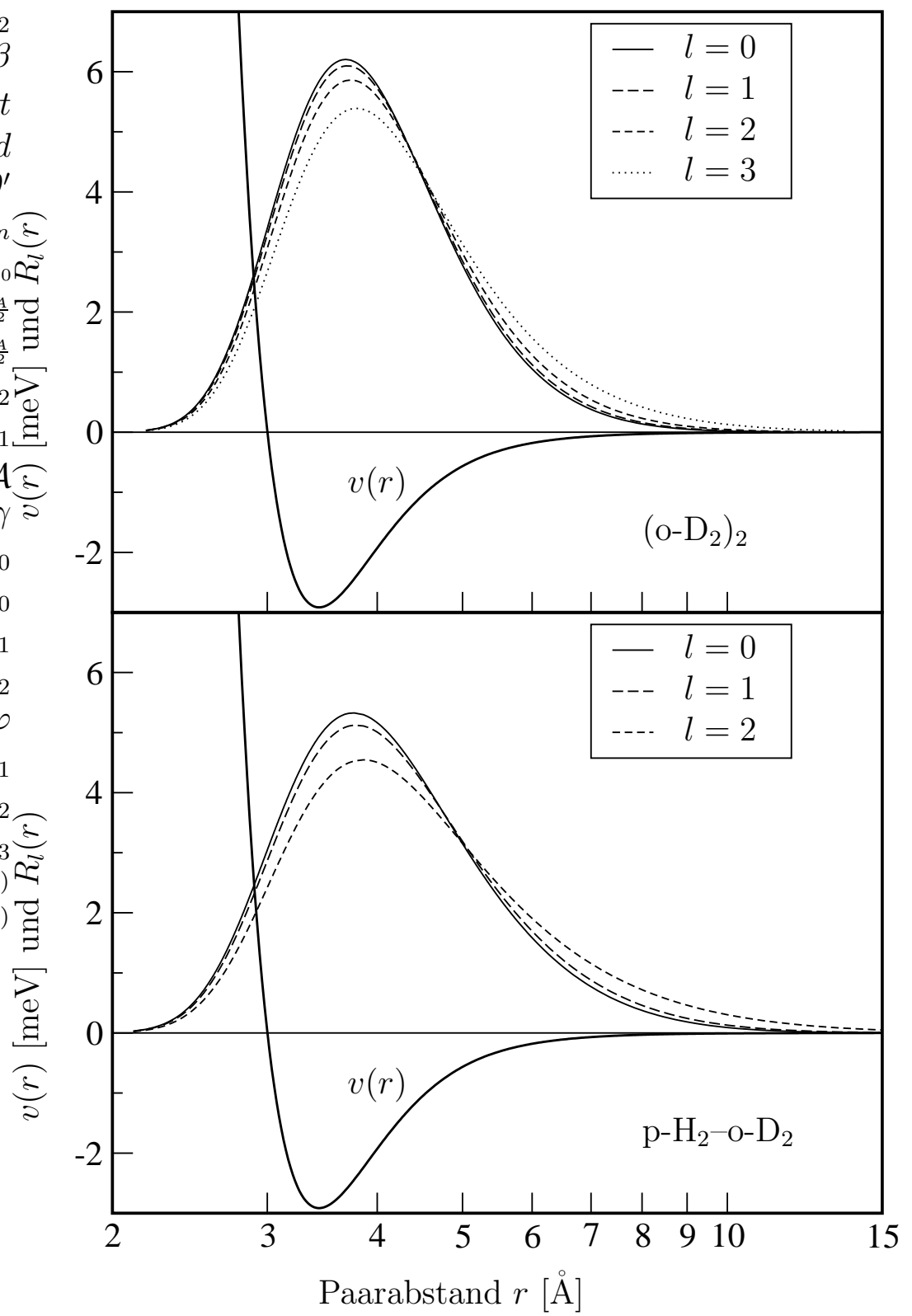

Abbildung 5.1: Halblogarithmische Auftragung der numerisch berechneten Radialwellenfunktionen $R_{l}(r)$ der Bindungszustände von (o- $\left.\mathrm{D}_{2}\right)_{2}$ (oben) und p- $\mathrm{H}_{2}-\mathrm{O}-\mathrm{D}_{2}$ (unten). Die Ordinate bezieht sich auf das Potential von Buck et al [80], das zum Vergleich eingetragen ist. 


\begin{tabular}{|cc|cc|}
\hline$\left(\mathrm{o}-\mathrm{D}_{2}\right)_{2}$ & $l$ & $E_{l}[\mu \mathrm{eV}]$ & $E_{l}[\mu \mathrm{eV}][74]$ \\
\hline & 0 & -848 & -754 \\
& 1 & -720 & -633 \\
& 2 & -471 & -396 \\
& 3 & -116 & -62 \\
\hline \hline $\mathrm{p}-\mathrm{H}_{2}-\mathrm{O}-\mathrm{D}_{2}$ & $l$ & $E_{l}[\mu \mathrm{eV}]$ & \\
\hline & 0 & -564 & \\
& 1 & -394 & \\
2 & -78 & \\
\hline \hline
\end{tabular}

Tabelle 5.1: Bindungsenergien der Zustände von $\left(\mathrm{o}-\mathrm{D}_{2}\right)_{2}$ und $\mathrm{p}-\mathrm{H}_{2}-\mathrm{O}-\mathrm{D}_{2}$ im Paarpotential (5.11). Alle Bindungszustände gehören zur Hauptquantenzahl $h=1$. Sie stellen somit die Rotationsanregungen der Dimere zur Vibrationsquantenzahl $\nu=0$ dar. In der rechten Spalte sind Literaturdaten für $\left(\mathrm{o}-\mathrm{D}_{2}\right)_{2}$ aus Ref. [74] zum Vergleich angegeben. Für p- $\mathrm{H}_{2}-\mathrm{O}-\mathrm{D}_{2}$ wurden keine vergleichbaren Daten gefunden, sein Spektrum findet sich aber in Ref. [41].

beim $\mathrm{p}-\mathrm{H}_{2}-\mathrm{O}-\mathrm{D}_{2}$. Diese Dimere sind also räumlich wesentlich kompakter als das Helium-Dimer mit seinem circa zehnfach größeren Paarabstand.

Analog kann auch das Wasserstoff-Dimer $\left(\mathrm{p}-\mathrm{H}_{2}\right)_{2}$ behandelt werden. Allerdings besitzt es nur zwei Bindungszustände zu den Drehimpulsquantenzahlen $l=0,1$. Da das $\left(\mathrm{p}-\mathrm{H}_{2}\right)_{2}$ aus ununterscheidbaren Teilchen besteht, gilt für seine Beugung am Transmissionsgitter die Auswahlregel (5.8), die den paritätsverändernden Übergang $0 \rightarrow 1$ verbietet. Zur Untersuchung inelastischer Beugung ist das $\left(\mathrm{p}-\mathrm{H}_{2}\right)_{2}$ daher nicht geeignet.

\subsubsection{Beugungsbilder von $\left(\mathrm{o}-\mathrm{D}_{2}\right)_{2}$ und p- $\mathrm{H}_{2}-\mathrm{o}-\mathrm{D}_{2}$}

Für die Beugungsbilder werden realistische Parameter gewählt. Die Geometrie des Transmissionsgitters, wie es in den Kapiteln 2-4 charakterisiert wurde, wird folgendermaßen angesetzt: $s_{0}=600 \AA, S_{0}=1350 \AA, \varphi=0,56 \mathrm{rad}$ und $\beta=5,8^{\circ}$. Für die Wechselwirkungskonstante $C_{3}$ zwischen $\mathrm{D}_{2}$ und Silizium-Nitrid wird ein experimentell bestimmter Wert verwendet: $C_{3}=0,32 \mathrm{meV} \mathrm{nm}^{3}$ [18]. Der entsprechende Wert für $\mathrm{H}_{2}$ scheint in der Literatur nicht bekannt zu sein. Er muß daher abgeschätzt werden: Einer Arbeit von Hoinkes [83] zufolge ist $C_{3}$ für schwach polarisierbare Atome und Moleküle näherungsweise proportional zu deren statischer elektrischer Dipolpolarisierbarkeit. Die Polarisierbarkeiten von $\mathrm{D}_{2}$ und $\mathrm{H}_{2}$ unterscheiden sich aber nur um circa $1 \%$. Aus diesem Grund wird für das $\mathrm{H}_{2}$-Molekül ebenfalls $C_{3}=0,32 \mathrm{meV} \mathrm{nm}{ }^{3}$ verwendet.

Als mittlere Strahlgeschwindigkeit wird $v^{\prime}=500 \mathrm{~ms}^{-1}$ gewählt und eine typische Geschwindigkeitsverteilung von $\Delta v^{\prime} / v^{\prime}=8 \%$ zugelassen $[18,62]$. Dies entspricht einer mittleren kinetischen Energie von $5,2 \mathrm{meV}$ pro $\mathrm{D}_{2}$-Molekül beziehungsweise 2,6 meV für $\mathrm{H}_{2}$ sowie einer Temperatur von $T_{b}=m\left(\Delta v^{\prime}\right)^{2} / 2 k_{\mathrm{B}} \approx 0,4 \mathrm{~K}$ [62] im mitbewegten Bezugssystem des Strahls. Rotationsanregungen der Moleküle können also, wie oben vorausgesetzt, vernachlässigt werden. Die relativen Besetzungszahlen $p_{l^{\prime} m^{\prime}}$ der Rota- 
tionszustände der Dimere in Gleichung (5.10) stellen sich während der Expansionsund Abkühlungsphase des Strahls nach dem Austritt aus der Düse ein. Es wird angenommen, daß die energetisch entarteten Zustände zu festem $l^{\prime}$ aber verschiedenen $m^{\prime}$ mit gleicher Wahrscheinlichkeit gebildet werden. Für die Zustände zu unterschiedlichen $l^{\prime}$ wird eine Gewichtung mit der Strahltemperatur $p_{l^{\prime} m^{\prime}} \propto \exp \left(-E_{l^{\prime}} / k_{\mathrm{B}} T_{b}\right)$ angenommen, wobei $k_{\mathrm{B}}$ die Boltzmann-Konstante bezeichnet. Wegen der $m^{\prime}$-Entartung ist es sinnvoll, die Besetzungszahl der Zustände zu gleichem $l^{\prime}$ zu definieren durch

$$
p_{l^{\prime}}:=\frac{1}{Z} \sum_{m^{\prime}=-l^{\prime}}^{l^{\prime}} \exp \left(-E_{l^{\prime}} / k_{\mathrm{B}} T_{b}\right)=\frac{1}{Z}\left(2 l^{\prime}+1\right) \exp \left(-E_{l^{\prime}} / k_{\mathrm{B}} T_{b}\right),
$$

wobei die Normierungskonstante $Z$ so gewählt ist, daß $\sum_{l^{\prime}} p_{l^{\prime}}=1$ gilt.

In einem typischen Beugungsexperiment kommt es aufgrund der endlichen Strahldivergenz und der Breite des Eingangsspalts des Detektors zu einer Verbreiterung der Beugungsmaxima. Eine zusätzliche, von der Beugungsordnung $n$ abhängige Verbreiterung entsteht durch die Geschwindigkeitsverteilung $\Delta v^{\prime}$ [56]. Diese Effekte sind einerseits abhängig von der verwendeten experimentellen Apparatur, andererseits ist das spezielle Profil der verbreiterten Beugungsmaxima im allgemeinen nicht von Belang. Aus diesem Grund wird für die Verbreiterung ein empirisches Modell verwendet: aus früheren Arbeiten $[5,18,19]$ ist bekannt, daß sich ein beim Beugungswinkel $\theta_{n}$ befindliches $n$. Maximum der Intensität $I_{n}$ in sehr guter Näherung durch eine Gausssche Glockenkurve der Gestalt

$$
\frac{I_{n}}{\sqrt{\pi} w_{n}} \exp \left(-\frac{\left(\theta-\theta_{n}\right)^{2}}{w_{n}^{2}}\right)
$$

repräsentieren läßt, deren Breite $w_{n}$ nach der Formel

$$
w_{n}=w_{0} \sqrt{1+\left(\frac{\Delta w}{w_{0}} n\right)^{2}}
$$

von der Beugungsordnung $n$ abhängt. Darin bezeichnet die Konstante $w_{0}$ die Breite der nullten Beugungsordnung, und $\Delta w$ bestimmt die ordnungsabhängige Verbreiterung für $n \neq 0$. Für die numerischen Rechnungen werden die Erfahrungswerte $w_{0}=3 \times 10^{-3}$ Grad und $\Delta w=7 \times 10^{-4}$ gewählt.

\section{Beugung von $\left(o-D_{2}\right)_{2}$}

Zunächst wird das Beugungsbild eines reinen Strahls von $\left(\mathrm{o}-\mathrm{D}_{2}\right)_{2}$-Dimeren untersucht. Aufgrund der niedrigen Strahltemperatur $T_{b}$ befindet sich der Großteil der einlaufenden $\left(\mathrm{o}-\mathrm{D}_{2}\right)_{2}$ im Grundzustand $l^{\prime}=0$. Nur circa $7 \%$ sind im Zustand $l^{\prime}=1$, und die Besetzungszahlen der Zustände zu $l^{\prime}=2$ und $l^{\prime}=3$ sind vernachlässigbar klein (vgl. Tab. 5.2a). Da das $\left(\mathrm{o}^{-} \mathrm{D}_{2}\right)_{2}$ aus ununterscheidbaren Teilchen besteht, sind der Auswahlregel (5.8) zufolge neben den elastischen Übergängen nur die inelastischen Übergänge $0 \rightarrow 2$ und $1 \rightarrow 3$ (Anregung) sowie die umgekehrten Prozesse $2 \rightarrow 0$ 


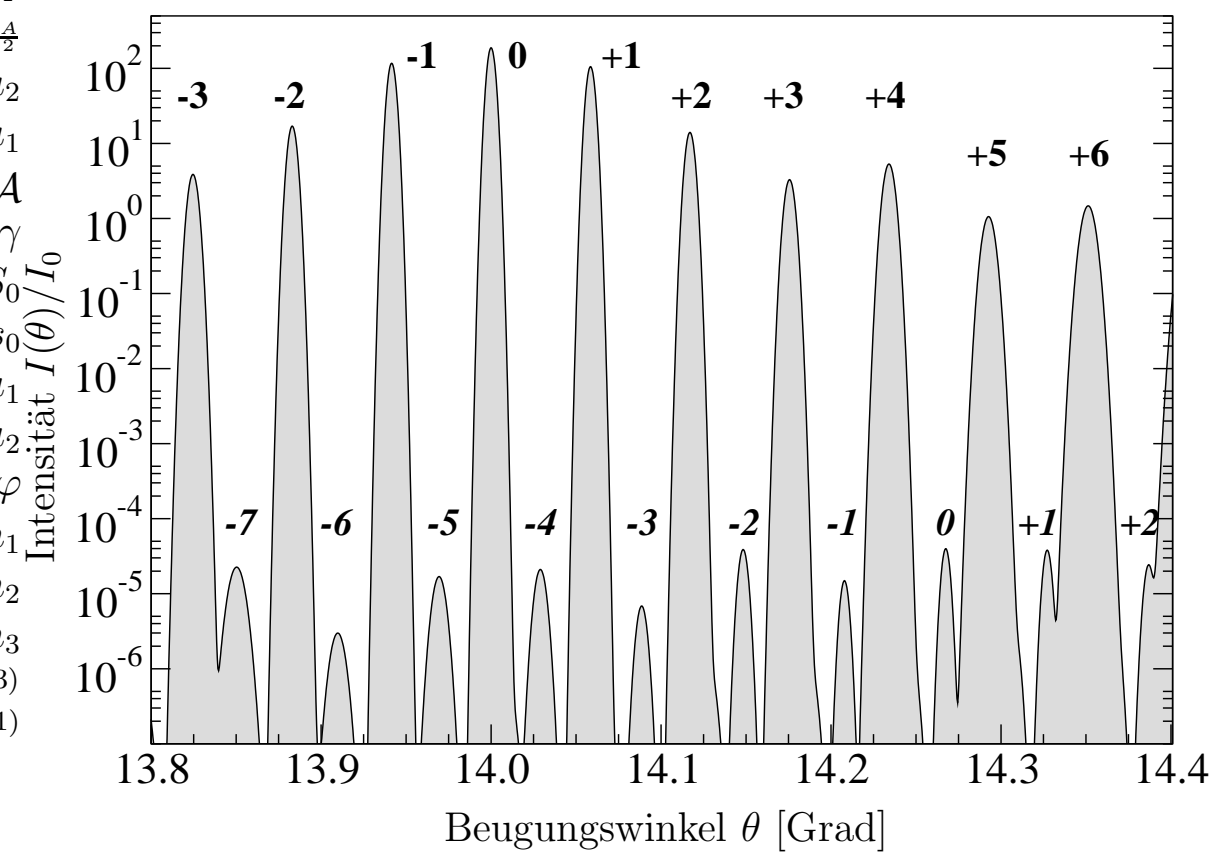

Abbildung 5.2: Beugungsbild eines reinen $\left(\mathrm{o}-\mathrm{D}_{2}\right)_{2}$-Strahls beim Einfallswinkel $\theta^{\prime}=14^{\circ}$. Die intensiven Maxima rühren von der elastischen Beugung aller einlaufenden Zustände her. Die schwächeren Maxima, deren Beugungsordnungen kursiv numeriert sind, stammen vom Übergang $0 \rightarrow 2$. Maxima weiterer Übergänge werden überdeckt oder sind zu schwach, um aufgelöst zu werden. Das Beugungsbild ist normiert auf die Gesamtintensität der nullten Ordnung elastischer Beugung, $I_{0}:=I_{0}^{00}+I_{0}^{11}+I_{0}^{22}+I_{0}^{33}$.

a)

\begin{tabular}{|cc|}
\hline$l^{\prime}$ & $p_{l^{\prime}}$ \\
\hline 0 & 0,932 \\
1 & 0,068 \\
2 & $8 \times 10^{-5}$ \\
3 & $4 \times 10^{-9}$ \\
\hline
\end{tabular}

b)

\begin{tabular}{|cc|}
\hline$l^{\prime} \rightarrow l$ & $E_{l}-E_{l^{\prime}}[\mu \mathrm{eV}]$ \\
\hline elastisch $\left(l^{\prime}=l\right)$ & 0 \\
$0 \rightarrow 2$ & 377 \\
$1 \rightarrow 3$ & 604 \\
\hline \hline
\end{tabular}

Tabelle 5.2: $a$ ) Besetzungswahrscheinlichkeiten der einlaufenden Zustände von $\left(\mathrm{o}-\mathrm{D}_{2}\right)_{2}$ bei $T_{b}=0,4 \mathrm{~K} . b$ ) Erlaubte Anregungen und Übergangsenergien. Die entsprechenden Abregungen mit negativen Übergangsenergien sind ebenfalls möglich. 
und $3 \rightarrow 1$ (Abregung) erlaubt. Die Übergangsenergien sind in Tabelle 5.2b zusammengefaßt.

In Abbildung 5.3 ist das nach den Gleichungen (5.10) und (5.14) berechnete Beugungsbild des reinen $\left(\mathrm{o}-\mathrm{D}_{2}\right)_{2}$-Strahls dargestellt. Neben den elastischen Beugungsmaxima sind Maxima des inelastischen Übergangs $0 \rightarrow 2$ erkennbar. Die Abstände dieser inelastischen Beugungsmaxima entsprechen der Übergangsenergie von $E_{2}-E_{0}=377 \mu \mathrm{eV}$. Inelastische Maxima anderer Übergänge sind aufgrund der geringen Besetzungswahrscheinlichkeit der höheren Bindungszustände nicht mehr auflösbar. Die Hierarchie der Maxima hängt von der Geometrie des Gitters und der attraktiven Wechselwirkung ab.

\section{Beugung von $\mathrm{p}-\mathrm{H}_{2}-\mathbf{O}-\mathrm{D}_{2}$}

Als zweites Beispiel wird das Beugungsbild eines reinen Strahls von p- $\mathrm{H}_{2}-\mathrm{o}-\mathrm{D}_{2}$ untersucht. Da die Teilchen dieses Dimers unterscheidbar sind, kommt die Auswahlregel (5.8) nicht zur Anwendung; alle Übergänge sind erlaubt. Wiederum befindet sich die

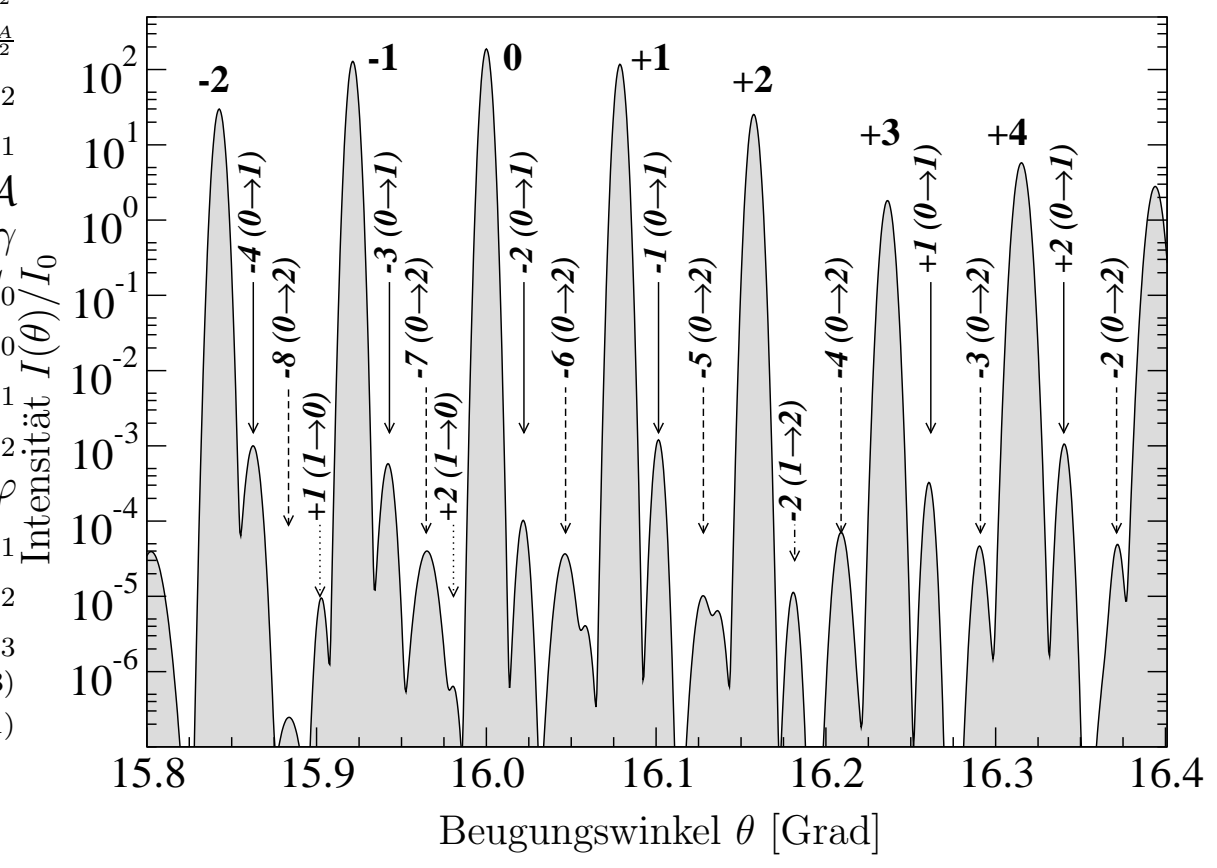

Abbildung 5.3: Beugungsbild eines reinen p- $\mathrm{H}_{2}-\mathrm{O}-\mathrm{D}_{2}$-Strahls beim Einfallswinkel $\theta^{\prime}=$ $16^{\circ}$. Die starken Maxima rühren von elastischer Beugung her. Darüberhinaus können die inelastischen Maxima der Übergänge $0 \rightarrow 1$ und $0 \rightarrow 2$ aufgelöst werden sowie einige weitere Übergänge, die von $l^{\prime}=1$ ausgehen. Das Beugungsbild ist normiert auf die Gesamtintensität der nullten Ordnung elastischer Beugung, $I_{0}:=I_{0}^{00}+I_{0}^{11}+I_{0}^{22}$.

Mehrzahl der Dimere im Grundzustand. Nur circa 2\% der Besetzungswahrscheinlichkeit entfallen auf den Zustand $l^{\prime}=1$, und die Besetzung des Zustandes $l^{\prime}=2$ ist vernachlässigbar (vgl. Tab. 5.3a). In Abbildung 5.3 ist das Beugungsbild dargestellt. Da der Übergang $0 \rightarrow 1$ erlaubt ist, ist neben den elastischen Maxima eine Vielfalt inelastischer Maxima zu beobachten, die vom einlaufenden Zustand $l^{\prime}=0$ 
a)

\begin{tabular}{|cc|}
\hline$l^{\prime}$ & $p_{l^{\prime}}$ \\
\hline 0 & 0,979 \\
1 & 0,021 \\
2 & $4 \times 10^{-6}$ \\
\hline
\end{tabular}

b)

\begin{tabular}{|cc|}
\hline$l^{\prime} \rightarrow l$ & $E_{l}-E_{l^{\prime}}[\mu \mathrm{eV}]$ \\
\hline elastisch $\left(l^{\prime}=l\right)$ & 0 \\
$0 \rightarrow 1$ & 170 \\
$0 \rightarrow 2$ & 486 \\
$1 \rightarrow 2$ & 316 \\
\hline \hline
\end{tabular}

Tabelle 5.3: a) Besetzungswahrscheinlichkeiten der einlaufenden Zustände von p- $\mathrm{H}_{2}-\mathrm{o}-\mathrm{D}_{2}$ bei $T_{b}=0,4 \mathrm{~K} . b$ ) Anregungen und Übergangsenergien. Die entsprechenden Abregungen mit negativen Übergangsenergien sind ebenfalls möglich.

ausgehen. Ihre Abstände entsprechen den Übergangsenergien $E_{1}-E_{0}=170 \mu \mathrm{eV}$ und $E_{2}-E_{0}=486 \mu \mathrm{eV}$ (vgl. Tab. 5.3b). Darüberhinaus können einige von $l^{\prime}=1$ ausgehende inelastische Maxima zugeordnet werden. Weiterhin ist zu beobachten, daß die nullte Ordnung des paritätsverändernden Übergangs $0 \rightarrow 1$ stark unterdrückt ist; im hypothetischen Fall verschwindender attraktiver Wechselwirkung ist ihre Intensität sogar Null. Die Unterdrückung rührt daher, daß die atomaren Transmissionsfunktionen der als Punktteilchen betrachteten Moleküle $\mathrm{H}_{2}$ und $\mathrm{D}_{2}$ näherungsweise spiegelsymmetrisch bezüglich ihrer Ortsargumente sind und sich außerdem aufgrund der gleichen Wechselwirkungskonstante $C_{3}$ sehr ähnlich sind.

\subsubsection{Experimentelle Realisierbarkeit inelastischer Beugung}

Die mit realistischen experimentellen Parametern berechneten Beugungsbilder zeigen, daß es prinzipiell möglich ist, die inelastische Beugung von van der Waals-Clustern quantitativ auszuwerten und dadurch Teile des Energie-Spektrums der Cluster $\mathrm{zu}$ bestimmen. Aufgrund des aus den Abbildungen 5.2 und 5.3 ablesbaren Intensitätsverhältnisses von circa $10^{-5}$ der inelastischen zu den elastischen Maxima werden jedoch relativ hohe Ansprüche an die Rauscharmut und die zeitliche Stabilität der Meßapparatur gestellt. Sie liegen im Grenzbereich des Auflösungsvermögens des derzeit von Brühl, Kornilov, Kalinin und Toennies verwendeten Elektronenionisationsdetektors.

Abschließend sollte noch einmal betont werden, daß zwar die Intensitäten der Beugungsmaxima vom betrachteten Cluster abhängen, da sie durch die Übergangswahrscheinlichkeit zwischen verschiedenen Bindungszuständen des Clusters bestimmt werden. Die übrigen Ergebnisse, wie die Formel für die Beugungswinkel und die Auswahlregel, gelten aber unabhängig von der Form der Bindungswellenfunktionen ebenso für Dimere wie Trimere und größere Cluster. Dies sollte sich für Experimente als vorteilhaft erweisen, da die (angeregten) Bindungswellenfunktionen sowie die Beugung bereits für einfachste Vierteilchensysteme wie das Helium-Tetramer nur unter großem analytischen und numerischen Aufwand berechnet werden können $[23,33,84,85]$. 


\section{Kapitel 6}

\section{Zusammenfassung und Ausblick}

In den vergangenen Jahren ist es technisch möglich geworden, präzise Beugungsexperimente mit Atom-, Molekular- und Clusterstrahlen durchzuführen. Eine wichtige experimentelle Voraussetzung dafür ist die Verfügbarkeit hochgenauer, nanostrukturierter Transmissionsgitter, die die kohärente Streuung an vielen Gitterstegen erlauben. Diese Gitter können gegenwärtig routinemäßig gefertigt werden [14]. Eine weitere wichtige Voraussetzung ist eine quantitative Theorie der Teilchenbeugung, die die Interpretation von Meßdaten erst ermöglicht. Diese Theorie muß sowohl die schwache van der Waals-Wechselwirkung zwischen den Teilchen und dem Gitter als auch, im Falle von Clustern, deren innere Struktur und die möglichen Streukanäle einschließlich des Aufbruchkanals berücksichtigen. Eine solche Theorie wurde von Hegerfeldt und Köhler [3, 4] auf der Grundlage der quantenmechanischen Streutheorie für schwach gebundene, zweiatomige Cluster (Dimere) entwickelt. In der vorliegenden Arbeit wurde diese Theorie für die Beugung des nächstkomplizierteren Clusters, des Trimers, weiterentwickelt und erfolgreich für die Auswertung von Experimenten benutzt. Im Vergleich mit der Dimer-Beugung konnten Regelmäßigkeiten erkannt werden, die zum allgemeinen physikalischen Verständnis der Clusterbeugung beitragen.

Kapitel 2 befaßte sich mit der Theorie der Atombeugung bei nicht-senkrechtem Einfall und stellte eine Vorarbeit für alle folgenden Kapitel dar. Diese war nötig, da die derzeit genauesten verfügbaren Beugungsdaten für das Helium-Dimer und -Trimer von Brühl, Kalinin, Kornilov und Toennies bei nicht-senkrechtem Einfall aufgenommen wurden, um die Empfindlichkeit zu erhöhen. Darüberhinaus kommen die Eigenheiten der inelastischen Beugung von Clustern erst bei nicht-senkrechtem Einfall vollständig zur Geltung. Es stellte sich heraus, daß das Beugungsbild von Atomen unter nicht-senkrechtem Einfall auf zwei charakteristische Arten asymmetrisch ist. Die eine Ausprägung der Asymmetrie ist rein geometrischer Natur und wäre auch bei der optischen Beugung zu beobachten. Der andere Beitrag zur Asymmetrie rührt vom unterschiedlich starken Einfluß der van der Waals-Wechselwirkung der Atome mit den beiden einen Spalt begrenzenden Stegen her. Die Theorie gibt die Asymmetrie korrekt wieder.

In Kapitel 3 wurde die Beugungstheorie für Trimere entwickelt. Dabei zeigte

sich zunächst, daß die abstrakte Formulierung der Streumatrix und der Kanal-Über- 
gangsoperatoren formal derjenigen der Dimerbeugung gleicht, wenn auch die zugrundeliegenden Hilberträume verschieden sind. Die daraufhin störungstheoretisch unter der Annahme geringer Bindungsenergie durchgeführte Ableitung der Übergangsamplitude des Trimers führte auf halbem Wege der Rechnung zu sehr umfangreichen Formeln, die jedoch durch Ausnutzung von Symmetrieeigenschaften der Trimer-Bindungszustände vereinfacht und quantitativ mit sehr guter Genauigkeit approximiert werden konnten. Die Trimer-Übergangsamplitude ließ sich schließlich durch eine Gitterfunktion und eine Spaltamplitude ausdrücken; die Transmissionsfunktion des Trimers konnte physikalisch interpretiert werden. Es zeigte sich, daß das aus früheren Arbeiten bekannte Konzept der „effektiven Spaltbreite“ auch bei der Trimerbeugung einsetzbar ist. Dieses Konzept bringt die van der Waals-Wechselwirkung mit dem Transmissionsgitter sowie die räumliche Ausdehnung der Cluster mit einer scheinbaren Verringerung der Gitterspaltbreite in Verbindung. Als relevante Meßgröße stellte sich dabei die mittlere Projektion der drei Paarabstände des Trimers auf das Transmissionsgitter heraus. Dieses Resultat war anschaulich verständlich und gab im Vergleich mit den entsprechenden Resultaten der Atom- und Dimerbeugung auch einen Hinweis darauf, wie Beugungsbilder größerer Cluster zu interpretieren sein könnten. Unter zusätzlichen Voraussetzungen, die beispielsweise beim Helium-Trimer erfüllt sind, erwies sich darüberhinaus in guter Näherung der Paarabstand selbst als Meßgröße.

Im 4. Kapitel wurde die Beugungstheorie der vorangehenden Kapitel zur Auswertung der Experimente zur Atom-, Dimer- und Trimerbeugung von Brühl, Kalinin, Kornilov und Toennies verwendet. Die ursprüngliche Hoffnung, den angeregten Zustand des Helium-Trimers untersuchen zu können, zerschlug sich bald, da in den experimentellen Daten kein signifikanter Hinweis auf seine Gegenwart im HeliumStrahl gefunden werden konnte; eine Abschätzung der relativen Besetzungszahlen zeigte, daß wahrscheinlich nur circa ein Prozent der Helium-Trimere im angeregten Zustand vorlag, der Rest im Grundzustand. Die folgende Auswertung konzentrierte sich deshalb auf die Untersuchung des Grundzustandes, wobei der Nachweis seines theoretisch vorhergesagten Paarabstandes von nur $\langle r\rangle=9,6 \AA$ [32] hohe Anforderungen an die Genauigkeit und die Stabilität der Meßapparatur stellte. Aus drei Meßserien bei unterschiedlichen Einfallswinkeln konnten für $\langle r\rangle$ die Werte 12,0 (+5/ - 8) $\AA$, $10,0(+5 /-7) \AA$ und $18,5( \pm 2,5) \AA$ ermittelt werden, wovon ersteren beiden ein höheres Gewicht beigemessen wurde. Die Fehlerangaben sind ein Maß für das gegenwärtige Auflösungsvermögen der Apparatur, wobei der Gewinn an Empfindlichkeit durch nicht-senkrechten Einfall auf eine Halbierung der Fehlerangaben geschätzt wurde. Außerdem wurden die zugehörigen Meßserien für das Helium-Dimer untersucht und ein Paarabstand von $\langle r\rangle=48(+3 /-8) \AA$ ermittelt. Ein Teil dessen negativer Fehlerangabe war die Folge eines notwendigen Näherungsschrittes. Dieser wurde erstmals durch eine weitesgehend modellunabhängige Vorgehensweise theoretisch korrigiert. Das verbesserte Ergebnis lautet $\langle r\rangle=43(+3 /-3) \AA$.

Schließlich wurde in Kapitel 5 der interessante aber bislang experimentell unberücksichtigte Aspekt der inelastischen Beugung von Clustern am Beispiel der van der Waals-Dimere $\left(\mathrm{o}-\mathrm{D}_{2}\right)_{2}$ und $\mathrm{p}-\mathrm{H}_{2}-\mathrm{O}-\mathrm{D}_{2}$ quantitativ untersucht. Die Anregung innerer Zustände der Cluster durch die Wechselwirkung mit dem Transmissionsgitter führt zu 
zusätzlichen, charakteristischen Beugungsmaxima. Aus den zugehörigen Beugungswinkeln kann ähnlich der Analyse eines Spektrums in der Spektroskopie die Übergangsenergie der Anregung berechnet werden. Die quantitative Analyse zeigte, daß die Winkelauflösung mit dem gegenwärtig von Brühl, Kalinin, Kornilov und Toennies verwendeten Detektor keine Schwierigkeit darstellt. Somit eignet sich ein experimenteller Aufbau zur inelastischen Beugung auch zur Erzeugung eines Clusterstrahls mit definierten inneren Zuständen. Allerdings befindet sich die Anforderung an die Rauscharmut derzeit im Grenzbereich des Möglichen. Da die Beugung am Transmissionsgitter prinzipiell sehr genaue Messungen ermöglicht, ist diese Art der ClusterSpektroskopie mit einer verbesserten Detektionsapparatur ein erfolgversprechendes physikalisches Experiment und Hilfsmittel.

Für die nahe Zukunft der Beugung von Helium-Trimeren stellt sich insbesondere die Frage nach einem effizienten Anregungsmechanismus zur Erzeugung einer nachweisbaren Besetzungszahl des Efimov-Zustandes des ${ }^{4} \mathrm{He}_{3}$. Der Nachweis dieses in der theoretischen Literatur nahezu einstimmig vorhergesagten Zustandes würde den ersten experimentellen Hinweis auf den eigenartigen Efimov-Effekt geben, dessen Diskussion seit seiner Entdeckung durch Efimov [38] im Jahr 1970 nicht an Interesse eingebüßt hat. Die Kenntnis der Daten beider Bindungszustände von ${ }^{4} \mathrm{He}_{3}$ würde es auf theoretischer Seite ermöglichen, universelle Skalierungseigenschaften schwach gebundener Dreiteilchensysteme zu überprüfen [35], die wiederum zum Verständnis der Bildung von Bose-Einstein-Kondensaten kleiner Cluster von Interesse sind [39].

Eine Aufgabe, der am Schluß von Kapitel 3 wegen des gegenwärtig untergeordneten experimentellen Interesses nicht nachgegangen wurde, ist die Vereinfachung des geometrischen Anteils der effektiven Spaltbreite (und somit die Interpretation experimenteller Daten) für Trimere, die aus unterscheidbaren Teilchen bestehen. Es ist naheliegend anzunehmen, daß sich die Summe der projizierten Paarabstände in entsprechender Weise wie bei identischen Teilchen durch die Paarabstände selbst ausdrücken läßt. Außerdem blieben Trimere, deren Zweiteilchen-Streumatrizen nicht in der unitären Polapproximation durch die niedrigste Partialwellenkomponente angenähert werden können, sowie Trimere zum Gesamtdrehimpuls $L>0$ in diesem letzten Rechnungsschritt unberücksichtigt. Eine weitergehende Betrachtung der FaddeevGleichungen entsprechend dem Vorgehen in Anhang B sollte hier auf die benötigten Zusammenhänge führen.

Sowohl in der Dimer- als auch in der Trimerbeugung bestehen die Übergangsamplituden aus der kohärenten Überlagerung mehrerer Streuterme, die als Vorwärts-, Einfach- und Zweifachstreuterme sowie Dreifachstreuterme (nur Trimer) interpretiert wurden. In beiden Fällen tragen aber nur der Vorwärts- und der Zweifachstreuterm (Dimer) beziehungsweise der Dreifachstreuterm (Trimer) wesentlich zum Beugungsbild bei, wohingegen sich die übrigen, wie durch eine relativ aufwendige Berechnung gezeigt wird, unter der Voraussetzung der Beugungsbedingung nahezu kompensieren. Es ist bislang kein grundsätzliches Hindernis zu erkennen, weshalb dieses Verhalten nicht auch bei größeren Clustern auftreten sollte. Diese Beobachtung könnte es ermöglichen, in sehr guter Näherung die allgemeine Struktur der Übergangsamplitude für $N$-Teilchen-Cluster anzugeben. Größere Helium-Cluster erlangten kürzlich Aufmerksamkeit nach der Entdeckung möglicher "magischer Zahlen“ 
bei $N \approx 10,15,26,44$ und 85 Atomen [86].

Ein weiteres, sehr interessantes Gebiet stellt die Erweiterung der Beugungsapparatur zu einem vollständigem Materiewellen-Interferometer mit drei Transmissionsgittern dar. Die Interferometrie zählt zu den genauesten bekannten Meßverfahren (z. B. Ref. [87]). Das Funktionsprinzip des Materiewellen-Interferometers wurde bereits auf verschiedene Weise demonstriert [16, 88], und ein funktionsfähiges MachZehnder-Interferometer wurde von Brühl und Toennies konstruiert und zur Messung der statischen elektrischen Dipolpolarisierbarkeit von Helium und dem Helium-Dimer eingesetzt [89]. Es besteht die Hoffnung, dieses Instrument auch zur genaueren Untersuchung schwach gebundener Cluster einsetzen zu können. Da wegen der geringen Abstände der drei Transmissionsgitter von nur einigen Zentimetern die FraunhoferNäherung der Resolvente nicht mehr zulässig ist, muß eine korrekte Theorie auch das Nahfeld der Materiewellen beschreiben. 


\section{Anhang A}

\section{Attraktive Wechselwirkung}

\section{A.1 Die Transmissionsfunktion}

\section{A.1.1 Die Phasenfunktion bei nicht-senkrechtem Einfall}

Aus früheren Experimenten [5, 18] ist bekannt, daß der attraktiven van der WaalsWechselwirkung zwischen den Teilchen im Strahl und dem beugenden Objekt bei der quantitativen Auswertung experimenteller Daten Rechnung getragen werden muß. Dazu wurden in Kapitel 2 die Transmissionsfunktionen des Einzelstegs $\tau_{a}^{\text {at }}\left(\boldsymbol{p}^{\prime} ; a_{2}\right)$ (2.28) beziehungsweise des Spalts $\tau_{u}^{\text {at }}\left(\boldsymbol{p}^{\prime} ; u_{2}\right)$ (2.32) eingeführt. Letztere wurde in der Form

$$
\tau_{u}^{\text {at }}\left(\boldsymbol{p}^{\prime} ; u_{2}\right)=\exp \left[\mathrm{i} \phi_{u}\left(\boldsymbol{p}^{\prime} ; u_{2}\right)\right] \quad \text { für } \quad-\frac{S_{0}}{2}<u_{2}<\frac{S_{0}}{2}
$$

geschrieben, wobei die Phasenfunktion $\phi_{u}\left(\boldsymbol{p}^{\prime} ; u_{2}\right)$ laut ihrer Definition (2.33) durch

$$
\phi_{u}\left(\boldsymbol{p}^{\prime} ; u_{2}\right)=-\left.\frac{m}{\left|\boldsymbol{p}^{\prime}\right| \hbar} \int \mathrm{d} x_{\|} W_{\mathrm{att}}(\mathbf{x})\right|_{x_{\perp}=\left[\frac{\pi_{1}+\pi_{1}^{\prime}}{p_{a_{1}+p_{a_{1}}^{\prime}}}\left(u_{2} \mp \frac{S_{0}}{2}\right) \mp \frac{A}{2}\right] \sin \left(\gamma+\theta^{\prime}\right)}
$$

zu berechnen ist. Hier ist das obere Vorzeichen im Intervall $0<u_{2}<\frac{S_{0}}{2}$ zu verwenden und das untere in $-\frac{S_{0}}{2}<u_{2}<0$. Mit Hilfe der Transformationen (2.21) und (2.29) kann das darin auftretende Verhältnis von Impulskomponenten näherungsweise berechnet werden:

$$
\frac{\pi_{1}+\pi_{1}^{\prime}}{p_{a_{1}}+p_{a_{1}}^{\prime}}=\frac{\sin \left(\varphi-\theta^{\prime}\right)}{\sin \left(\gamma+\theta^{\prime}\right)}\left[1+\mathcal{O}\left(\frac{p_{\perp}+p_{\perp}^{\prime}}{p_{\|}+p_{\|}^{\prime}}\right)\right]
$$

Die vernachlässigten Korrekturterme sind von der Größenordnung $10^{-3}$. Folglich darf das Potential $W_{\text {att }}(\mathbf{x})$ näherungsweise bei $x_{\perp}=\left(u_{2} \mp \frac{S_{0}}{2}\right) \sin \left(\varphi-\theta^{\prime}\right) \mp \frac{A}{2} \sin \left(\gamma+\theta^{\prime}\right)$ ausgewertet werden. Eine geometrische Überlegung zeigt, daß dies genau dann der Fall ist, wenn die Variable $u_{2}$ den Schnittpunkt der $u_{2}$-Achse mit dem Integrationsweg von Gleichung (A.2) beschreibt (vgl. Abb. A.1). Dies ist das erwartete Ergebnis. Der Integrationsweg wird im folgenden in parametrisierter Form in der Ebene durch die 
lineare Funktion

$$
\begin{aligned}
& \boldsymbol{g}\left(s ; u_{2}\right)=\boldsymbol{g}\left(0 ; u_{2}\right)+s \boldsymbol{p}^{\prime} /\left|\boldsymbol{p}^{\prime}\right| \text { mit } \\
& \boldsymbol{g}\left(0 ; u_{2}\right)=\left(u_{2}-\frac{S_{0}}{2}\right)\left[\cos \varphi \boldsymbol{e}_{1}+\sin \varphi \boldsymbol{e}_{2}\right]+\frac{s_{0}}{2} \boldsymbol{e}_{2}
\end{aligned}
$$

dargestellt, wobei $\boldsymbol{e}_{1}$ und $\boldsymbol{e}_{2}$ die üblichen (zweidimensionalen) Einheitsvektoren bezeichnen. Die Phasenfunktion lautet daraufhin

$$
\phi_{u}\left(\boldsymbol{p}^{\prime} ; u_{2}\right)=-\frac{1}{\hbar v^{\prime}} \int_{-\infty}^{\infty} \mathrm{d} s W_{\text {att }}\left(\boldsymbol{g}\left(s ; u_{2}\right)\right) \quad \text { mit } \quad v^{\prime}=\frac{\left|\boldsymbol{p}^{\prime}\right|}{m} .
$$

Für die attraktive Wechselwirkung zwischen einem Atom im Teilchenstrahl und

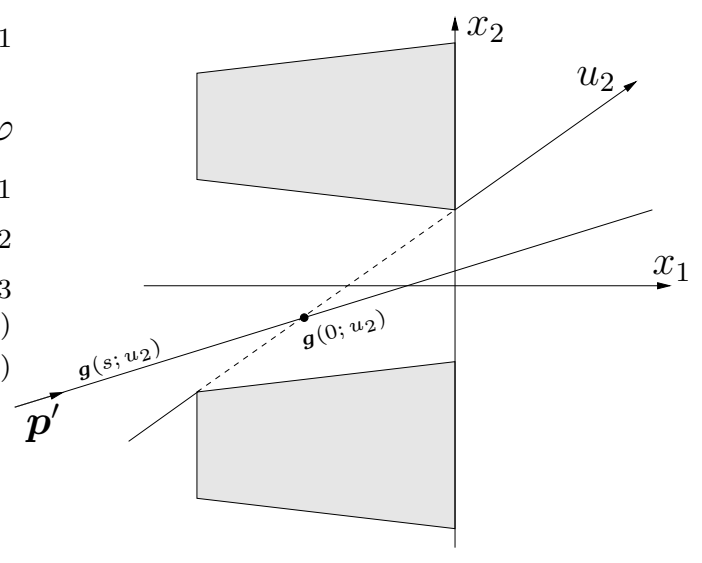

Abbildung A.1: Skizze zur Berechnung der Transmissionsfunktion $\tau_{u}^{\text {at }}$ in der Eikonalapproximation

einem kleinen Volumenelement des Steges wird zunächst ein übliches Lennard-JonesPotential angenommen,

$$
-\frac{C_{6}}{l^{6}}+\frac{C_{12}}{l^{12}}
$$

wobei $l$ den Abstand bezeichnet und $C_{6}$ und $C_{12}$ Konstanten sind, die die Stärke der Wechselwirkung beschreiben. Die genaue Form des repulsiven Anteils ist allerdings nicht von Bedeutung. Er wird bereits durch die Randbedingungen bei der Behandlung der Atombeugung (vgl. Kap. 2.2.1 und Ref. [40]) modelliert und daher im folgenden nicht berücksichtigt. Die Phasenfunktion läßt sich nun durch vierdimensionale Integration von $-C_{6} / l^{6}$ über das Volumen des Stegs sowie entlang der Geraden $\boldsymbol{g}\left(s ; u_{2}\right)$ berechnen,

$$
\phi_{u}\left(\boldsymbol{p}^{\prime} ; u_{2}\right)=\frac{C_{6}}{\hbar v^{\prime}} \int_{-\infty}^{\infty} \mathrm{d} s \int_{-t}^{0} \mathrm{~d} x_{1}^{\prime} \int_{y_{u}\left(x_{1}^{\prime}\right)}^{y_{o}\left(x_{1}^{\prime}\right)} \mathrm{d} x_{2}^{\prime} \int_{-\infty}^{\infty} \mathrm{d} x_{3}^{\prime}\left[\left|\boldsymbol{x}^{\prime}-\boldsymbol{g}\left(s ; u_{2}\right)\right|^{2}+x_{3}^{\prime 2}\right]^{-3},
$$

wobei $\boldsymbol{x}^{\prime}$ die zweidimensionale Projektion von $\mathbf{x}^{\prime}$ in die $\left(x_{1}, x_{2}\right)$-Ebene bezeichnet. Die Funktionen $y_{u}\left(x_{1}^{\prime}\right)$ und $y_{o}\left(x_{1}^{\prime}\right)$ repräsentieren hier die Flanken des Steges. Im Spalt, der durch zwei Stege begrenzt wird, setzt sich $\phi_{u}$ additiv aus den Beiträgen 
beider Stege zusammen. Für den „oberen“ Steg in Abbildung A.1 lauten die Integrationsgrenzen $y_{u}\left(x_{1}^{\prime}\right)=\frac{s_{0}}{2}-x_{1}^{\prime} \tan \beta$ und $y_{o}\left(x_{1}^{\prime}\right)=d-\frac{s_{0}}{2}+x^{\prime} \tan \beta$; für den "unteren" Steg lauten sie $y_{u}\left(x_{1}^{\prime}\right)=-d+\frac{s_{0}}{2}-x^{\prime} \tan \beta$ und $y_{o}\left(x_{1}^{\prime}\right)=-\frac{s_{0}}{2}+x^{\prime} \tan \beta$. Das Integral (A.7) läßt sich elementar lösen, indem die Integrationsreihenfolge vertauscht wird und zunächst die Integrale über $x_{3}^{\prime}$ und $s$ ausgeführt werden. Unter Verwendung der geometrischen Relationen

$$
\begin{aligned}
& S_{0} \cos \varphi=t \\
& S_{0} \sin \varphi=s_{0}+t \tan \beta
\end{aligned}
$$

und der Abkürzungen

$$
C_{3}:=\frac{\pi C_{6}}{6}, \quad \widetilde{d}:=\frac{d \cos \theta^{\prime}}{\sin \left(\varphi-\theta^{\prime}\right)}, \quad \widetilde{s_{0}}:=\frac{s_{0} \cos \theta^{\prime}}{\sin \left(\varphi-\theta^{\prime}\right)},
$$

erhält man

$$
\begin{gathered}
\phi_{u}\left(\boldsymbol{p}^{\prime} ; u_{2}\right)=\frac{C_{3}}{2 \hbar v^{\prime} \cos ^{4} \theta^{\prime}} \frac{1}{\cos ^{2} \varphi\left(\tan \varphi-\tan \theta^{\prime}\right)^{2}} \\
\times\left\{\frac { 1 } { \operatorname { t a n } \theta ^ { \prime } - \operatorname { t a n } \beta } \left[\left(\frac{S_{0}}{2}+u_{2}\right)^{-2}-\left(\frac{S_{0}}{2}-u_{2}-\widetilde{s}\right)^{-2}\right.\right. \\
\left.+\left(\frac{S_{0}}{2}+u_{2}-\widetilde{d}\right)^{-2}-\left(\frac{S_{0}}{2}-u_{2}-\widetilde{s}+\widetilde{d}\right)^{-2}\right] \\
+\frac{1}{\tan \theta^{\prime}+\tan \beta}\left[\left(\frac{S_{0}}{2}-u_{2}\right)^{-2}-\left(\frac{S_{0}}{2}-u_{2}-\widetilde{s}+\frac{\tan \varphi+\tan \theta^{\prime}}{\tan \varphi-\tan \theta^{\prime}} S_{0}\right)^{-2}\right. \\
\left.\left.+\left(\frac{S_{0}}{2}-u_{2}-\widetilde{d}\right)^{-2}-\left(\frac{S_{0}}{2}-u_{2}-\widetilde{s}+\frac{\tan \varphi+\tan \theta^{\prime}}{\tan \varphi-\tan \theta^{\prime}} S_{0}-\widetilde{d}\right)^{-2}\right]\right\} .
\end{gathered}
$$

Die scheinbaren Singularitäten bei $\theta^{\prime}= \pm \beta$ sind hebbar. Dieser zunächst unübersichtlich anmutende Ausdruck wird sehr vereinfacht, wenn der einfallende Strahl einen ausreichend großen Winkel mit beiden Stegflanken einschließt (bei $\beta=6,2^{\circ}$ sind beispielsweise $\theta^{\prime}=10^{\circ}$ genügend). Unter dieser Bedingung trägt aus den beiden eckigen Klammernpaaren in (A.9) nur jeweils der erste Term nennenswert bei, und es folgt näherungsweise

$$
\phi_{u}\left(\boldsymbol{p}^{\prime} ; u_{2}\right) \approx \lambda_{-}\left(\frac{S_{0}}{2}+u_{2}\right)^{-2}+\lambda_{+}\left(\frac{S_{0}}{2}-u_{2}\right)^{-2}
$$

mit den von $\boldsymbol{p}^{\prime}$ abhängigen Vorfaktoren

$$
\lambda_{ \pm}:=\frac{C_{3}}{2 \hbar v^{\prime} \cos ^{4} \theta^{\prime}} \frac{1}{\cos ^{2} \varphi\left(\tan \varphi-\tan \theta^{\prime}\right)^{2}} \frac{1}{\tan \theta^{\prime} \pm \tan \beta} .
$$

Das Potenzgesetz (A.10), dem die Phasenfunktion gehorcht, ist charakteristisch für die geometrische Form des Objektes und das Paarpotential. 
Es bleibt zu prüfen, ob die Transmissionsfunktion die in Kapitel 2.2.1 geforderte Bedingung $\tau_{u}^{\text {at }}\left(\boldsymbol{p}^{\prime} ; 0\right) \approx 1$ in der Spaltmitte erfüllt. Für die in Abbildung 2.5 gezeigte Messung findet man beispielsweise $\lambda_{+}=1444 \AA^{2}$ und $\lambda_{-}=2717 \AA^{2}$. Zusammen mit der experimentell bestimmten Spaltbreite $S_{0}=1397,5 \AA$ folgt aus Gleichung (A.10) für die Phasenfunktion $\phi_{u}\left(\boldsymbol{p}^{\prime} ; 0\right) \approx 9 \times 10^{-3}$. Die Transmissionsfunktion erfüllt die Bedingung also in guter Näherung. Allerdings ist die Bedingung beispielsweise nicht erfüllt, wenn an Stelle von Helium-Atomen im Grundzustand hoch angeregte, metastabile Helium-Atome verwendet werden, deren Wechselwirkungskonstante $C_{3}$ circa um einen Faktor 40 größer ist [90].

\section{A.1.2 Die Phasenfunktion bei senkrechtem Einfall}

Für die Auswertung einiger früherer experimenteller Meßserien zur Trimer-Beugung, die bei senkrechtem Einfall durchgeführt wurden, wurde die zugehörige Transmissionsfunktion $\tau_{x}^{\text {at }}\left(\boldsymbol{p}^{\prime} ; x_{2}\right)$ benötigt. Diese ist bekannt [5, 18] und wird hier der Vollständigkeit halber in derselben Form wie ihr Pendant bei nicht-senkrechtem Einfall $\tau_{u}^{\text {at }}\left(\boldsymbol{p}^{\prime} ; u_{2}\right)$ angegeben. Für $0<\theta^{\prime}<\beta$ kann sie geschrieben werden als

$$
\tau_{x}^{\text {at }}\left(\boldsymbol{p}^{\prime} ; x_{2}\right)= \begin{cases}\exp \left[\mathrm{i} \phi_{x}\left(\boldsymbol{p}^{\prime} ; x_{2}\right)\right] & :-\frac{s_{0}}{2}<x_{2}<\frac{s_{0}}{2} \\ 0 & : \text { sonst }\end{cases}
$$

Die Phasenfunktion $\phi_{x}\left(\boldsymbol{p}^{\prime} ; x_{2}\right)$ läßt sich aufgrund der Abschätzung (A.3) unter Berücksichtigung der geometrischen Relationen (A.8) durch Einsetzen der Transformation $u_{2}=\frac{\frac{1}{2} s_{0}+x_{2}-t\left(\tan \theta^{\prime}-\tan \beta\right)}{s-t\left(\tan \theta^{\prime}-\tan \beta\right)} S_{0}$ ohne erneute Berechnung direkt aus Gleichung (A.9) gewinnen. Allgemein findet man für $0<\theta^{\prime}<\beta$

$$
\begin{aligned}
& \phi_{x}\left(\boldsymbol{p}^{\prime} ; x_{2}\right)=\frac{C_{3}}{2 \hbar v^{\prime} \cos ^{4} \theta^{\prime}} \\
& \times\left\{\frac { 1 } { \operatorname { t a n } \theta ^ { \prime } - \operatorname { t a n } \beta } \left[\left(\frac{s_{0}}{2}+x_{2}-t\left(\tan \theta^{\prime}-\tan \beta\right)\right)^{-2}-\left(\frac{s_{0}}{2}+x_{2}\right)^{-2}\right.\right. \\
& \left.+\left(\frac{s_{0}}{2}+x_{2}-t\left(\tan \theta^{\prime}-\tan \beta\right)-d\right)^{-2}-\left(\frac{s_{0}}{2}+x_{2}-d\right)^{-2}\right] \\
& +\frac{1}{\tan \theta^{\prime}+\tan \beta}\left[\left(\frac{s_{0}}{2}-x_{2}\right)^{-2}-\left(\frac{s_{0}}{2}-x_{2}+t\left(\tan \theta^{\prime}+\tan \beta\right)\right)^{-2}\right. \\
& \left.\left.+\left(\frac{s_{0}}{2}-x_{2}-d\right)^{-2}-\left(\frac{s_{0}}{2}-x_{2}+t\left(\tan \theta^{\prime}+\tan \beta\right)-d\right)^{-2}\right]\right\} .
\end{aligned}
$$

Die approximative Darstellung der Phasenfunktion als Potenzgesetz lautet bei senkrechtem Einfall $\theta^{\prime}=0$ einfach

$$
\phi_{x}\left(\boldsymbol{p}^{\prime} ; x_{2}\right) \approx \lambda\left\{\left(\frac{s_{0}}{2}+x_{2}\right)^{-2}+\left(\frac{s_{0}}{2}-x_{2}\right)^{-2}\right\}
$$

mit $\lambda:=C_{3} / 2 \hbar v^{\prime} \tan \beta$. 


\section{A.1.3 Asymptotik der Kumulanten der Atombeugung}

Die effektive Spaltbreite der Atombeugung (2.76) steht in einer charakteristischen, approximativen Relation zur mittleren Geschwindigkeit $v^{\prime}$ der Atome, wenn die Phasenfunktion als Potenzgesetz in der Form von Gleichung (A.10) dargestellt werden kann. Zur Ableitung dieser Relation wird zunächst das asymptotische Verhalten der atomaren Kumulanten $R_{1}^{ \pm}$für kleine Werte der Wechselwirkungskonstanten $\lambda_{ \pm}$untersucht. Stellvertretend wird die Kumulante $R_{1}^{+}$betrachtet. Sie lautet Gleichung (2.62) zufolge

$$
R_{1}^{+}=\frac{S_{0}}{2}-\int_{0}^{S_{0} / 2} \mathrm{~d} \xi \tau_{u}^{\text {at }}\left(\boldsymbol{p}^{\prime} ; \frac{S_{0}}{2}-\xi\right) .
$$

Wegen der kurzen Reichweite der attraktiven Wechselwirkung braucht der Beitrag des bei $u_{2}=-\frac{S_{0}}{2}$ liegenden Steges im Integrationsintervall nicht berücksichtigt zu werden. Als eine Verallgemeinerung wird darüberhinaus zunächst eine beliebige Potenz $-\nu$ mit $\nu>1$ in der Phasenfunktion (A.10) angenommen.

$$
\phi_{u}\left(\boldsymbol{p}^{\prime} ; u_{2}\right)=\lambda_{+}\left(\frac{S_{0}}{2}-u_{2}\right)^{-\nu}, \quad 0<u_{2}<\frac{S_{0}}{2}
$$

Die Forderung, daß die Transmissionsfunktion in der Spaltmitte $\tau_{u}^{\text {at }}\left(\boldsymbol{p}^{\prime} ; 0\right) \approx 1$ erfüllen soll, bedeutet, daß

$$
\delta_{+}:=\phi_{u}\left(\boldsymbol{p}^{\prime} ; 0\right)=\lambda_{+}\left(\frac{S_{0}}{2}\right)^{-\nu} \ll 1
$$

ein kleiner Parameter ist. Ausgehend von Gleichung (A.15) folgen mit der Substitution $z:=\lambda_{+} \xi^{-\nu}$ die Rechenschritte

$$
\begin{aligned}
R_{1}^{+}-\frac{S_{0}}{2} & =-\int_{0}^{S_{0} / 2} \mathrm{~d} \xi \exp \left(\mathrm{i} \lambda_{+} \xi^{-\nu}\right)=\int_{\delta_{+}}^{\infty} \mathrm{d} z \frac{\mathrm{d} \xi}{\mathrm{d} z} \exp (\mathrm{i} z) \\
& \stackrel{\text { P.I. }}{=}[\xi(z) \exp (\mathrm{i} z)]_{\delta_{+}}^{\infty}-\mathrm{i} \int_{\delta_{+}}^{\infty} \mathrm{d} z \xi(z) \exp (\mathrm{i} z) \\
& =-\frac{S_{0}}{2} \exp \left(\mathrm{i} \delta_{+}\right)-\mathrm{i} \lambda_{+}^{\frac{1}{\nu}}\left\{\int_{0}^{\infty} \mathrm{d} z z^{-\frac{1}{\nu}} \exp (\mathrm{i} z)-\int_{0}^{\delta_{+}} \mathrm{d} z z^{-\frac{1}{\nu}} \exp (\mathrm{i} z)\right\} .
\end{aligned}
$$

Während das erste der beiden Integrale in den geschweiften Klammern eine bekannte Konstante liefert [91],

$$
\int_{0}^{\infty} \mathrm{d} z z^{-\frac{1}{\nu}} \exp (\mathrm{i} z)=\Gamma\left(\frac{\nu-1}{\nu}\right) \exp \left(\mathrm{i} \pi \frac{\nu-1}{2 \nu}\right),
$$

wird das zweite durch

$$
\int_{0}^{\delta_{+}} \mathrm{d} z z^{-\frac{1}{\nu}} \exp (\mathrm{i} z)=\frac{\nu}{\nu-1} \delta_{+}^{1-\frac{1}{\nu}}+\mathcal{O}\left(\delta_{+}^{2-\frac{1}{\nu}}\right)
$$

ersetzt. Für die Kumulante $R_{1}^{+}$folgt bis einschließlich der linearen Ordnung in $\delta_{+}$

$$
R_{1}^{+}=\frac{S_{0}}{2}\left[-\mathrm{i} \delta_{+}^{\frac{1}{\nu}} \Gamma\left(\frac{\nu-1}{\nu}\right) \exp \left(\mathrm{i} \pi \frac{\nu-1}{2 \nu}\right)+\frac{1}{\nu-1} \mathrm{i} \delta_{+}+\mathcal{O}\left(\delta_{+}^{2}\right)\right],
$$


und speziell für den Fall $\nu=2$ gilt, unter Verwendung von $\Gamma\left(\frac{1}{2}\right)=\sqrt{\pi}$,

$$
R_{1}^{+}=\frac{S_{0}}{2}\left[(1-\mathrm{i}) \sqrt{\frac{\pi}{2} \delta_{+}}+\mathrm{i} \delta_{+}+\mathcal{O}\left(\delta_{+}^{2}\right)\right] .
$$

Analog zu $R_{1}^{+}$wird $R_{1}^{-}$berechnet. Während der Imaginärteil von $R_{1}^{+}$bereits in linearer Ordnung in $\delta_{+}$eine Korrektur zum führenden Term aufweist, ist die nächste nicht-verschwindende Ordnung im Realteil erst quadratisch. Aus diesem Grund gehorcht die auf $\operatorname{Re} R_{1}^{ \pm}$basierende effektive Spaltbreite $S_{\text {eff }}$ (2.66) asymptotisch besser einem Potenzgesetz als die auf $\operatorname{Im} R_{1}^{ \pm}$basierenden Größen $\Delta$ (2.67) und $\alpha$ (2.68). Ersetzt man in Gleichung (A.17) $\delta_{+}$wieder durch $\lambda_{+}$, so erhält man allgemein die effektive Spaltbreite

$$
S_{\text {eff }}\left(v^{\prime}, \theta^{\prime}\right) \sim S_{0}-\Gamma\left(\frac{\nu-1}{\nu}\right) \cos \left(\frac{\pi}{2 \nu}\right)\left(\lambda_{+}^{\frac{1}{\nu}}+\lambda_{-}^{\frac{1}{\nu}}\right) .
$$

Nach der Definition (A.11) sind die Koeffizienten $\lambda_{ \pm}$invers proportional zur Geschwindigkeit $v^{\prime}$. Ein Potenzgesetz der Form (A.16) führt also auf das charakteristische asymptotische Verhalten $S_{0}-S_{\text {eff }}\left(v^{\prime}, \theta^{\prime}\right) \propto C_{3} v^{\prime-\frac{1}{\nu}}$. Die grafische Auftragung der experimentell bestimmten Werte für die effektive Spaltbreite gegen $v^{\prime-\frac{1}{\nu}}$ erlaubt daher die Überprüfung der im Modell gewählten Gittergeometrie. Speziell für die in dieser Arbeit betrachteten Experimente mit $\nu=2$ findet man

$$
S_{\text {eff }}\left(v^{\prime}, \theta^{\prime}\right) \sim S_{0}-\sqrt{\frac{\pi}{2}}\left(\sqrt{\lambda_{-}}+\sqrt{\lambda_{+}}\right)
$$

und folglich asymptotisch

$$
S_{0}-S_{\mathrm{eff}}\left(v^{\prime}, \theta^{\prime}\right) \propto \frac{C_{3}}{\sqrt{v^{\prime}}} .
$$

Die Asymptotik der zweiten atomaren Kumulanten $R_{2}^{+}$(Gl. 2.63) kann auf ähnliche Weise berechnet werden und soll hier nicht detailliert dargestellt werden. Ausgehend von

$$
R_{2}^{+}=\left(\frac{S_{0}}{2}\right)^{2}-\left(R_{1}^{+}\right)^{2}-2 \int_{0}^{S_{0} / 2} \mathrm{~d} \xi \xi \tau_{u}^{\text {at }}\left(\boldsymbol{p}^{\prime} ; \frac{S_{0}}{2}-\xi\right)
$$

führt die Untersuchung des Integrals

$$
\int_{0}^{S_{0} / 2} \mathrm{~d} \xi \xi \exp \left(\mathrm{i} \lambda_{+} \xi^{-2}\right)
$$

auf den Term $\int_{\delta_{+}}^{\infty} \mathrm{d} z z^{-1} \cos z \sim-\gamma_{\mathrm{E}}-\ln \delta_{+}$, wobei $\gamma_{\mathrm{E}} \approx 0,5772$ die Euler-Konstante bezeichnet [92]. Zusammengefaßt ergibt sich

$$
R_{2}^{+}=\left(\frac{S_{0}}{2}\right)^{2}\left[\frac{\pi}{2} \delta_{+}+\mathrm{i} \delta_{+} \ln \delta_{+}+\mathrm{i}\left(\pi-1+\gamma_{\mathrm{E}}\right) \delta_{+}-\sqrt{2 \pi}(1+\mathrm{i}) \delta_{+}^{\frac{3}{2}}+\mathcal{O}\left(\delta_{+}^{2}\right)\right] .
$$

Eine entsprechende Gleichung gilt für $R_{2}^{-}$. Die Formeln (A.18) und (A.22) erlauben die Abschätzung der bei der Kumulantenentwicklung auftretenden Größen $S_{\text {eff }}, \Delta$, $\alpha, \Sigma$ und $\Omega$ (vgl. Gl. 2.66-2.71). 


\section{A.2 Korrekturen zur Geometrie der Gitterstege}

Die zur Berechnung der attraktiven Wechselwirkung verwendete Keilstumpfform des Gittersteges (Abb. A.1) stellt eine Idealisierung der tatsächlichen Geometrie dar. Durch den Herstellungsprozeß bedingt sind die Kanten der Stege keineswegs scharf, und besitzen die Flanken der Stege Rauhigkeiten. Dies ist an elektronenmikroskopischen Aufnahmen zu erkennen [58, Abb. 3]. Es wird daher im folgenden mit Hilfe zweier Modelle zur besseren Approximation der attraktiven Wechselwirkung der realen Gitterstege untersucht, ob die Idealisierung gerechtfertigt ist. Dazu kommt jeweils die asymptotische Relation für die effektive Spaltbreite (A.21) zur Anwendung.

Nicht betrachtet werden an dieser Stelle die bereits zuvor untersuchten, durch unregelmäßige Stegbreite [58, Abschnitt D] oder Korrugation der Stegkanten [90, Kap. 4.3] verursachten Effekte. Beide treten bereits im Falle der Beugung am rein repulsiven Gitter auf und sind daher nicht im Rahmen der Eikonalapproximation zu behandeln. Sie führen jeweils zu einem sogenannten Debye-Waller-Faktor, der die Intensitäten höherer Beugungsordnungen exponentiell dämpft.

\section{A.2.1 Abgerundete Stegkanten}

Laut Abschnitt A.1.1 wird die Phasenfunktion (A.9) bei den experimentell relevanten Einfallswinkeln von zwei Termen (A.10) dominiert. Sie können als Beiträge jener Kanten des „oberen“ und „unteren“ Gittersteges interpretiert werden, die den Strahl begrenzen. Um zu untersuchen, welchen Einfluß eine geringe Abrundung dieser Kanten auf das Beugungsbild hat, liegt es daher nahe, die Stege näherungsweise gänzlich durch Zylinder zu ersetzen und die Auswirkung auf die Phasenfunktion zu berechnen. Dazu wird ein vereinfachtes Modell betrachtet, bei dem der Integrationsweg $\boldsymbol{g}\left(s ; x_{2}\right)$ der Phasenfunktion im Abstand $D-R$ an einem Zylinder mit Radius $R$ entlang führt, dessen Achse parallel zur $x_{3}$-Achse verläuft (vgl. Abb. A.2). Die Phasenfunk-

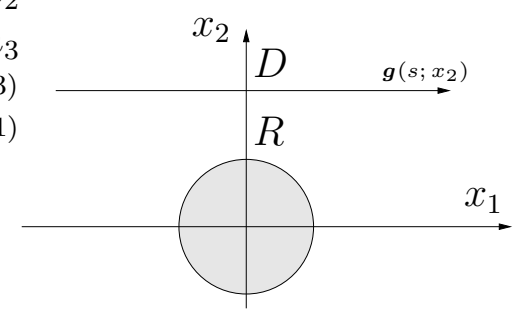

Abbildung A.2: Vereinfachtes Modell für einen Gittersteg mit abgerundeter Kante

tion kann entsprechend zu Gleichung (A.7) berechnet werden. Als exaktes Resultat erhält man

$$
\phi_{x}\left(\boldsymbol{p}^{\prime} ; D\right)=\frac{3 C_{3}}{\hbar v^{\prime}} \frac{\pi R^{2} D}{\left(D^{2}-R^{2}\right)^{3 / 2}},
$$

das für große Abstände $D-R \gg R$ wieder einem invers-quadratischen Potenzgesetz $\phi_{x}\left(\boldsymbol{p}^{\prime} ; D\right) \sim \lambda_{R}(D-R)^{-2}$ gehorcht, wobei $\lambda_{R}:=3 C_{3} \pi R^{2} / \hbar v^{\prime}$ eine Konstante bezeichnet. Für kleine Abstände $D-R \ll 4 R$ findet man hingegen $\phi_{x}\left(\boldsymbol{p}^{\prime} ; D\right) \sim$ $\lambda_{R}^{\prime}(D-R)^{-5 / 2}$ mit $\lambda_{R}^{\prime}:=3 C_{3} \pi \sqrt{2 R} / 8 \hbar v^{\prime}$. Betrachtet man einen Spalt, der von zwei 
derartigen Zylindern im Abstand $s_{0}$ begrenzt wird, so führt die Potenz $\nu=5 / 2$ bei kleinen Abständen auf eine Korrektur der asymptotischen Relation für die effektive Spaltbreite (A.21). Bei einem Zylinderradius der Größenordnung einiger Nanometer ist für die effektive Spaltbreite des betrachteten Modells bereits ein Verhalten von

$$
s_{0}-s_{\text {eff }}\left(v^{\prime}\right) \propto C_{3} v^{\prime-2 / 5}
$$

zu erwarten. Ein derartiges Potenzgesetz ist aber im Rahmen der experimentellen Unsicherheiten aus den Abbildungen 4.5-4.7 nicht erkennbar. Es wird daher angenommen, daß die Idealisierung der Stegkanten keinen systematischen Fehler in die Interpretation der Experimente einführt.

\section{A.2.2 Oberflächenrauhigkeit der Stegflanken}

Um den Einfluß der Oberflächenrauhigkeit der Stegflanken auf die attraktive Wechselwirkung abzuschätzen, wird ein einfaches Modell betrachtet. Die Stegoberfläche wird darin aufgeteilt in quadratische Felder der Kantenlänge $b$, denen jeweils eine Höhe $h_{n m}$ zugeordnet wird. Die Indizes $n m$ bezeichnen den Platz des Feldes. Es wird angenommen, daß die Höhen $h_{n m}$ normalverteilt sind, und im Mittel über alle Felder die Bedingungen $\left\langle h_{n m}\right\rangle=0$ und $\left\langle\left(h_{n m}\right)^{2}\right\rangle=\sigma^{2}$ erfüllen. Zur Berechnung von $W_{\text {att }}$ auf

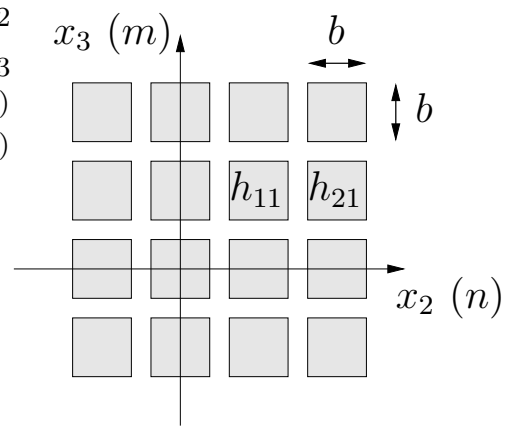

Abbildung A.3: Modell der Stegoberfläche mit Rauhigkeit $h_{n m}$ in der Richtung senkrecht zur Skizze.

Abständen $l$ von der Oberfläche, die gering sind gegen die Ausdehnung des Steges, darf der Einfachheit halber angenommen werden, daß der Steg im Halbraum $x_{1}<0$ unendlich ausgedehnt ist. Die attraktive Wechselwirkung an der Stelle $\mathbf{x}=(l, n b, m b)$ mit $l>0$ kann dann durch Integration von $-C_{6} / l^{6}$ über das Volumen des rauhen Steges berechnet werden. Unter Verwendung der Relation $C_{3}=\pi C_{6} / 6$ erhält man

$$
\begin{aligned}
W_{\text {att }}(\mathbf{x})=-\frac{C_{3}}{l^{3}}-C_{6} \sum_{n^{\prime}, m^{\prime}=-\infty}^{\infty} & \int_{\left(n^{\prime}-\frac{1}{2}\right) b}^{\left(n^{\prime}+\frac{1}{2}\right) b} \mathrm{~d} x_{2}^{\prime} \int_{\left(m^{\prime}-\frac{1}{2}\right) b}^{\left(m^{\prime}+\frac{1}{2}\right) b} \mathrm{~d} x_{3}^{\prime} \int_{0}^{h_{n^{\prime} m^{\prime}}} \mathrm{d} x_{1}^{\prime} \\
\times & {\left[\left(x_{1}^{\prime}-l\right)^{2}+\left(x_{2}^{\prime}-n b\right)^{2}+\left(x_{3}^{\prime}-m b\right)^{2}\right]^{-3}, }
\end{aligned}
$$

wobei der zweite Summand die durch die Rauhigkeit bedingte Abweichung von der glatten Oberfläche beschreibt. Für Abstände $l$, die groß sind gegen die Längenskala $\sigma$ der Rauhigkeit, kann der Integrand nach $x_{1}^{\prime} / l$ entwickelt und die Integration 
über $x_{1}^{\prime}$ ausgeführt werden. Nach Einführung der neuen Variablen $\widetilde{x}_{2}:=x_{2}^{\prime}-n^{\prime} b$; $\widetilde{x}_{3}:=x_{3}^{\prime}-m^{\prime} b ; \widetilde{n}:=n-n^{\prime} ; \widetilde{m}:=m-m^{\prime}$ und mit Hilfe der abkürzenden Schreibweise $a_{\widetilde{n} \widetilde{m}}^{2} \equiv\left(\widetilde{x}_{2}-\widetilde{n} b\right)^{2}+\left(\widetilde{x}_{3}-\widetilde{m} b\right)^{2}$ lautet die attraktive Wechselwirkung bis einschließlich der Ordnung $\mathcal{O}\left(h_{n m} / l\right)^{2}$ im Integranden

$$
\begin{aligned}
W_{\mathrm{att}}(\mathbf{x}) & \simeq-\frac{C_{3}}{l^{3}}-\frac{C_{6}}{l^{5}} \sum_{\widetilde{n}, \widetilde{m}=-\infty}^{\infty} \int_{-\frac{b}{2}}^{\frac{b}{2}} \mathrm{~d} \widetilde{x}_{2} \int_{-\frac{b}{2}}^{\frac{b}{2}} \mathrm{~d} \widetilde{x}_{3} \\
& \times\left\{\frac{h_{n-\widetilde{n} m-\widetilde{m}}}{l}\left[1+\left(\frac{a_{\widetilde{n} \widetilde{m}}}{l}\right)^{2}\right]^{-3}+3\left(\frac{h_{n-\widetilde{n} m-\widetilde{m}}}{l}\right)^{2}\left[1+\left(\frac{a_{\widetilde{n} \widetilde{m}}}{l}\right)^{2}\right]^{-4}\right\} .
\end{aligned}
$$

Die Integration der attraktiven Wechselwirkung über $\mathbf{x}=(l, n b, m b)$ zur Berechnung der Phasenfunktion (A.2) sowie die relativ große Höhe des Teilchenstrahls im Experiment kommen näherungsweise der Mittelung der attraktiven Wechselwirkung über viele Indizes $n m$ gleich. Wegen der angenommenen Verteilung der Höhen mit Erwartungswert $\left\langle h_{n m}\right\rangle=0$ sollte der erste Summand im Integranden dabei im Mittel vernachlässigbar sein. Im zweiten wird die Streuung $\sigma^{2}$ eingesetzt. Daraufhin kann das verbleibende Doppelintegral exakt gelöst werden. Man findet somit für die gemittelte attraktive Wechselwirkung

$$
\left\langle W_{\mathrm{att}}(\mathbf{x})\right\rangle \simeq-\frac{C_{3}}{l^{3}}\left[1+6 \frac{\sigma^{2}}{l^{2}}\right], \quad \sigma \ll l .
$$

Dieses einfache Ergebnis stimmt in erster Ordnung mit den aufwendigen Rechnungen von Marvin und Toigo überein [93, Abschnitt VI]. Die Rauhigkeit äußert sich also näherungsweise in einer Korrektur des Potenzgesetzes. Diese pflanzt sich in die Phasenfunktion fort. Sie führt dort zu einer Korrektur der invers-quadratischen Abstandsabhängigkeit (A.10), die wiederum eine Abweichung vom $v^{\prime-\frac{1}{2}}$-Verhalten der effektiven Spaltbreite (A.21) zur Folge hat. Dafür gibt es aber, wie bereits im Falle der abgerundeten Stegkanten, in den Daten für die effektive Spaltbreite im Rahmen der experimentellen Unsicherheiten keinen Beleg. Es wird daher angenommen, daß die Vernachlässigung der Oberflächenrauhigkeit nicht zu einer falschen Interpretation der experimentellen Daten führt. 


\section{Anhang B}

\section{Trimer-Bindungszustände}

Im Laufe von Kapitel 3 wurde gelegentlich Gebrauch gemacht von Eigenschaften der Trimer-Bindungswellenfunktionen, die nicht im Abschnitt 3.1 abgeleitet werden konnten, sowie von numerischen Rechnungen mit dem Ziel, Näherungsschritte quantitativ zu überprüfen. Die beiden wichtigsten davon betreffen die Ersetzung des Erwartungswerts $\left\langle\left|r_{\perp}\right|\right\rangle$ durch den halben Paarabstand $\frac{1}{2}\langle r\rangle$ sowie den Übergang von Gleichung (3.136) zu Gleichung (3.138) zur Approximation der effektiven Spaltbreite; wie die Diskussion auf Seite 71 zeigt, hängt die Güte dieser Approximationen von der konkreten Form der Bindungswellenfunktionen ab. Eine möglichst genaue Kenntnis der beiden Bindungswellenfunktionen des Helium-Trimers ist also wünschenswert. Allerdings sind diese selbst unter der Annahme, daß die potentielle Energie im Trimer allein aus zentralsymmetrischen Paarwechselwirkungen besteht und die Teilchen spinlos und ununterscheidbar sind, keineswegs einfach zu berechnen.

Die Behandlung des quantenmechanischen Dreiteilchenproblems im Umfeld der theoretischen Kernphysik warf mathematische Schwierigkeiten auf, die von Faddeev zu Beginn der sechziger Jahre des letzten Jahrhunderts durch die Formulierung eines Systems gekoppelter Integralgleichungen für den Dreiteilchenzustand überwunden wurden [94]. In den darauffolgenden Jahren wurde Faddeevs Zugang von vielen Autoren adoptiert, angewendet und erweitert (z. B. Ref. [49, 55, 95]). DreiteilchenBindungszustände sind spezielle Lösungen der homogenen Faddeev-Gleichungen zu negativer Gesamtenergie (im Schwerpunktsystem). Der in diesem Anhang verwendete Zugang zur Berechnung der Bindungszustände von Trimeren aus spinlosen, ununterscheidbaren Teilchen orientiert sich anfänglich an dem Buch von Sitenko [96, Kap. 10 u. 11]. Er führt auf eine eindimensionale Integralgleichung im Impulsraum, die anschließend numerisch gelöst werden kann. Entscheidend werden dabei die Annahme einer zentralsymmetrischen Paarwechselwirkung und anschließend die Näherung der Zweiteilchen-Streumatrix durch den niedrigsten Partialwellenanteil in der unitären Polapproximation nach Lovelace [95] sein.

Für anderweitige Zugänge zum quantenmechanischen Dreiteilchenproblem und seinen Bindungswellenfunktionen wird auf die Literaturübersicht in Abschnitt 3.1.3 verwiesen. 


\section{B.1 Spinlose, ununterscheidbare Teilchen}

\section{B.1.1 Die Faddeev-Gleichung}

Im Hinblick auf das Helium-Trimer wird nur der Fall spinloser, ununterscheidbarer Teilchen betrachtet. Bindungszustände des Trimers sind die normierbaren Lösungen der zeitunabhängigen Schrödinger-Gleichung

$$
\left[\frac{3}{4 m} \hat{\mathbf{q}}^{2}+\frac{1}{m} \hat{\mathbf{p}}^{2}+v\left(\left|\hat{\boldsymbol{\rho}}-\frac{1}{2} \hat{\mathbf{r}}\right|\right)+v(|\hat{\mathbf{r}}|)+v\left(\left|\hat{\boldsymbol{\rho}}+\frac{1}{2} \hat{\mathbf{r}}\right|\right)\right]|\phi\rangle=E|\phi\rangle .
$$

Im allgemeinen ist die Paarwechselwirkung $v(|\mathbf{r}|)$ nicht in analytischer Form bekannt oder liegt nur in Form einer komplizierten Reihenentwicklung vor, sodaß eine exakte Lösung der Schrödinger-Gleichung nicht möglich ist.

\section{Die Faddeev-Gleichung im Impulsraum}

Für die Zwecke der vorliegenden Arbeit eignen sich die Faddeev-Gleichungen [49], ein Satz von Integralgleichungen, der an die Stelle der Schrödinger-Gleichung (B.1) tritt. Eine Ableitung dieser Integralgleichungen wird beispielsweise von Sitenko [96, Kap. 11] gegeben. Im Fall ununterscheidbarer Teilchen reduzieren sie sich auf nur eine Gleichung. Mit Hilfe der unitären Transformation $\mathcal{U}\left(\mathbb{d}^{-1}\right)$ aus Gleichung (3.30), die im Impulsraum die explizite Darstellung

$$
\mathcal{U}\left(\mathbb{d}^{-1}\right) \psi(\mathbf{q}, \mathbf{p})=\psi\left(\left(\mathbb{d}^{-1}\right)^{T}\left(\begin{array}{l}
\mathbf{q} \\
\mathbf{p}
\end{array}\right)\right)
$$

besitzt, läßt sich jede Bindungswellenfunktion $\phi(\mathbf{q}, \mathbf{p}) \equiv\langle\mathbf{q}, \mathbf{p} \mid \phi\rangle$ des Trimers im Impulsraum in der Form

$$
\phi(\mathbf{q}, \mathbf{p})=\frac{1}{N}\left\{\varphi(\mathbf{q}, \mathbf{p})+\mathcal{U}\left(\mathbb{d}^{-1}\right) \varphi(\mathbf{q}, \mathbf{p})+\mathcal{U}\left(\mathbb{d}^{-1}\right)^{2} \varphi(\mathbf{q}, \mathbf{p})\right\}
$$

schreiben, wobei $N$ eine Normierungskonstante ist und die sogenannte Faddeev-Komponente $\varphi(\mathbf{q}, \mathbf{p})$ die Integralgleichung

$$
\varphi(\mathbf{q}, \mathbf{p})=\left[z_{q}-\frac{p^{2}}{m}\right]^{-1} \int \mathrm{d}^{3} p^{\prime}\left\langle\mathbf{p}\left|t\left(z_{q}\right)\right| \mathbf{p}^{\prime}\right\rangle\left[\mathcal{U}\left(\mathbb{d}^{-1}\right) \varphi\left(\mathbf{q}, \mathbf{p}^{\prime}\right)+\mathcal{U}\left(\mathbb{d}^{-1}\right)^{2} \varphi\left(\mathbf{q}, \mathbf{p}^{\prime}\right)\right]
$$

erfüllt [96]. Hier steht abkürzend $z_{q}:=E-\frac{3}{4} \frac{q^{2}}{m}+\mathrm{i} 0$, wobei $E$ die noch unbekannte Trimer-Bindungsenergie ist. Der Operator $t(z), z \in \mathbb{C}$, bezeichnet die Streumatrix eines Teilchenpaares,

$$
t(z):=v+v g(z) v,
$$

mit der zugehörigen Resolvente

$$
g(z):=\left[z-\frac{\hat{\mathbf{p}}^{2}}{2 \mu}-v\right]^{-1}
$$

und der reduzierten Masse des Paares $\mu:=m / 2$. Da die Faddeev-Gleichung (B.3) eine dreidimensionale Integralgleichung ist, ist ihre direkte numerische Lösung sehr aufwendig. Sie kann aber unter Ausnutzung der Symmetrien des Hamilton-Operators (B.1) sowie durch Approximation der Paarwechselwirkung $v(|\mathbf{r}|)$ vereinfacht werden. 


\section{Entwicklung der Zweiteilchen-Streumatrix nach Partialwellen}

Die Zentralsymmetrie der Paarwechselwirkung $v(|\mathbf{r}|)$ legt die Entwicklung des Matrixelements $\left\langle\mathbf{p}|t(z)| \mathbf{p}^{\prime}\right\rangle$ nach Eigenfunktionen der Drehimpulsoperatoren $\hat{l}^{2}$ und $\hat{l}_{3}$ des Teilchenpaares nahe. Zu diesem Zweck werden die Zustände $|p l m\rangle$ eingeführt. Mit den Definitionen $r:=|\mathbf{r}|$ und $\widehat{\mathbf{r}}:=\mathbf{r} / r$ lautet ihre Ortsraumdarstellung

$$
\langle\mathbf{r} \mid p l m\rangle:=\frac{4 \pi}{(2 \pi \hbar)^{3 / 2}} j_{l}\left(\frac{p r}{\hbar}\right) Y_{l}^{m}(\widehat{\mathbf{r}})
$$

wobei $j_{l}$ die $l$. sphärische Besselfunktion erster Art bezeichnet und $Y_{l}^{m}$ eine Kugelfächenfunktion ist. Durch Fourier-Transformation erhält man die ImpulsraumDarstellung

$$
\left\langle\mathbf{p}^{\prime} \mid p l m\right\rangle=(-\mathrm{i})^{l} p^{-2} \delta\left(p^{\prime}-p\right) Y_{l}^{m}\left(\widehat{\mathbf{p}}^{\prime}\right) .
$$

Sie erfüllen eine Orthogonalitätsrelation und eine Vollständigkeitsrelation:

$$
\begin{gathered}
\left\langle p^{\prime} l^{\prime} m^{\prime} \mid p l m\right\rangle=p^{-2} \delta\left(p^{\prime}-p\right) \delta_{l^{\prime} l} \delta_{m^{\prime} m} \\
\sum_{l=0}^{\infty} \sum_{m=-l}^{l} \int_{0}^{\infty} \mathrm{d} p p^{2}|p l m\rangle\langle p l m|=\mathbb{1} .
\end{gathered}
$$

Mit ihrer Hilfe kann das Impulsraum-Matrixelement der Paarwechselwirkung nach Legendre-Polynomen $P_{l}$ zerlegt werden,

$$
\left\langle\mathbf{p}|v| \mathbf{p}^{\prime}\right\rangle=: \sum_{l=0}^{\infty}(2 l+1) v_{l}\left(p, p^{\prime}\right) P_{l}\left(\widehat{\mathbf{p}} \cdot \widehat{\mathbf{p}^{\prime}}\right),
$$

wobei die $l$. Partialwellenkomponente des Paarpotentials durch die Formel

$$
v_{l}\left(p, p^{\prime}\right)=\frac{4 \pi}{(2 \pi \hbar)^{3}} \int_{0}^{\infty} \mathrm{d} r r^{2} j_{l}\left(\frac{p r}{\hbar}\right) v(r) j_{l}\left(\frac{p^{\prime} r}{\hbar}\right)
$$

gegeben ist. Wie an Gleichung (B.4) ersichtlich, überträgt sich die Zentralsymmetrie von $v$ auf die Zweiteilchen-Streumatrix, so daß auch sie nach Partialwellen entwickelt werden kann. Einsetzen des Ansatzes

$$
\left\langle\mathbf{p}|t(z)| \mathbf{p}^{\prime}\right\rangle=: \sum_{l=0}^{\infty}(2 l+1) t_{l}\left(p, p^{\prime} ; z\right) P_{l}\left(\widehat{\mathbf{p}} \cdot \widehat{\mathbf{p}^{\prime}}\right)
$$

in die Lippmann-Schwinger-Gleichung $t(z)=v+v g_{0}(z) t(z)$, wobei die freie Resolvente gegeben ist durch $g_{0}(z)=\left[z-\hat{\mathbf{p}}^{2} / 2 \mu\right]^{-1}$, führt auf eine Lippmann-SchwingerGleichung für die $l$. Partialwellenkomponente:

$$
t_{l}\left(p, p^{\prime} ; z\right)=v_{l}\left(p, p^{\prime}\right)+4 \pi \int_{0}^{\infty} \mathrm{d} \widetilde{p}^{2} v_{l}(p, \widetilde{p})\left[z-\frac{\widetilde{p}^{2}}{2 \mu}\right]^{-1} t_{l}\left(\widetilde{p}, p^{\prime} ; z\right)
$$


Durch Einsetzen der Zerlegung der Zweiteilchen-Streumatrix (B.11) in die Faddeev-Gleichung (B.3) folgt nun

$$
\begin{aligned}
\varphi(\mathbf{q}, \mathbf{p}) & =\left[z_{q}-\frac{p^{2}}{m}\right]^{-1} \int \mathrm{d}^{3} q^{\prime} \varphi\left(\mathbf{q}^{\prime}, \mathbf{q}+\frac{1}{2} \mathbf{q}^{\prime}\right) \\
& \times \sum_{l=0}^{\infty} 2 \Delta_{l}(2 l+1) t_{l}\left(p,\left|\mathbf{q}^{\prime}+\frac{1}{2} \mathbf{q}\right| ; z_{q}\right) P_{l}\left(\widehat{\mathbf{p}} \cdot\left(\widehat{\mathbf{q}^{\prime}+\frac{1}{2}} \mathbf{q}\right)\right)
\end{aligned}
$$

wobei der Faktor

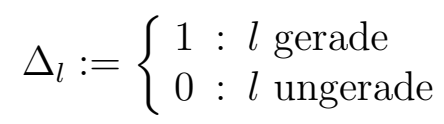

die Tatsache widerspiegelt, daß für spinlose, ununterscheidbare Teilchen nur gerade Zweiteilchen-Drehimpulse $l$ auftreten können.

\section{Entwicklung nach Eigenzuständen des Gesamtdrehimpulses}

Im freien Trimer ist der Gesamtdrehimpuls $\hat{\mathbf{L}}$ eine Erhaltungsgröße. Er setzt sich zusammen aus dem Drehimpuls $\hat{\mathbf{l}}$ eines Zweiteilchen-Untersystems und dem Drehimpuls $\hat{\lambda}$ des dritten Teilchens bezüglich des Schwerpunkts des Zweiteilchen-Untersystems $[96]$.

$$
\hat{\mathbf{L}}:=\hat{\lambda}+\hat{\mathbf{l}}
$$

Ein gemeinsames System von Basisfunktionen von $\hat{L}^{2}, \hat{L}_{3}$ und dem Hamilton-Operator (B.1) für die Winkelvariablen $\widehat{\mathbf{q}}$ und $\widehat{\mathbf{p}}$ ist die gekoppelte, vollständige und normierte Basis

$$
\mathcal{Y}_{\lambda l L M}(\widehat{\mathbf{q}}, \widehat{\mathbf{p}}):=\sum_{\mu=-\lambda}^{\lambda} \sum_{m=-l}^{l}(\operatorname{lm} \lambda \mu \mid L M) Y_{\lambda}^{\mu}(\widehat{\mathbf{q}}) Y_{l}^{m}(\widehat{\mathbf{p}}),
$$

wobei $(\operatorname{lm} \lambda \mu \mid L M)$ ein Clebsch-Gordan-Koeffizient [97] ist und $L$ und $M$ die Quantenzahlen von $\hat{L}^{2}$ beziehungsweise $\hat{L}_{3}$ bezeichnen. Die Faddeev-Komponente (B.13) kann zu festem $L$ und $M$ gewählt werden. Da $\hat{L}_{3}$ im Hamilton-Operator nicht auftritt, ist ihre Entwicklung nach den Funktionen $\mathcal{Y}_{\lambda l L M}$ von der Form

$$
\varphi_{L M}(\mathbf{q}, \mathbf{p})=: \sum_{\lambda=0}^{\infty} \sum_{l=0}^{\infty} \varphi_{\lambda l L}(q, p) \mathcal{Y}_{\lambda l L M}(\widehat{\mathbf{q}}, \widehat{\mathbf{p}}) .
$$

Einsetzen dieser Entwicklung in die Integralgleichung (B.13) und Ausnutzen der bekannten Orthogonalitätsrelationen der Kugelflächenfunktionen und Legendre-Polynome führt nach einigen Umformungen auf eine Integralgleichung für die Entwicklungskoeffizienten $\varphi_{\lambda l L}(q, p)[96]$.

$$
\begin{aligned}
& \varphi_{\lambda l L}(q, p)=\left[z_{q}-\frac{p^{2}}{m}\right]^{-1} 8 \pi \Delta_{l} \Delta_{l^{\prime}} \int_{0}^{\infty} \mathrm{d} q^{\prime} \int_{\left|q-\frac{1}{2} q^{\prime}\right|}^{q+\frac{1}{2} q^{\prime}} \mathrm{d} p^{\prime} \frac{q^{\prime} p^{\prime}}{q} \\
& \times t_{l}\left(p, \sqrt{p^{\prime 2}+\frac{3}{4} q^{\prime 2}-\frac{3}{4} q^{2}} ; z_{q}\right) \sum_{\lambda^{\prime}=0}^{\infty} \sum_{l^{\prime}=0}^{\infty} K_{\lambda l, \lambda^{\prime} l^{\prime}}^{(L)}\left(q, q^{\prime} ; p^{\prime}\right) \varphi_{\lambda^{\prime} l^{\prime} L}\left(q^{\prime}, p^{\prime}\right)
\end{aligned}
$$


Als Folge der Partialwellenzerlegung ist die Integralgleichung (B.17) zweidimensional, enthält aber unendliche Summen. Sämtliche winkelabhängigen Terme sind in dem Kern $K_{\lambda l, \lambda^{\prime} l^{\prime}}^{(L)}\left(q, q^{\prime} ; p^{\prime}\right)$ zusammengefaßt. Unter Verwendung der Transformationseigenschaften der Kugelfächenfunktionen unter Raumdrehungen und der Orthogonalitätsrelationen der Clebsch-Gordan-Koeffizienten [97, Kap. III und IV] können die Winkelintegrationen darin ausgeführt werden, und der Kern nimmt die einfache Form

$$
\begin{aligned}
& K_{\lambda l, \lambda^{\prime} l^{\prime}}^{(L)}\left(q, q^{\prime} ; p^{\prime}\right)=\frac{(4 \pi)^{3 / 2}}{\sqrt{2 \lambda^{\prime}+1}} \sum_{m=-l}^{l} \sum_{m^{\prime}=-l^{\prime}}^{l^{\prime}}(-1)^{l+l^{\prime}-m-m^{\prime}} \\
& \quad \times(l m L-m \mid \lambda 0)\left(l^{\prime} m^{\prime} L-m \mid \lambda^{\prime} m^{\prime}-m\right)\left(Y_{l}^{m}\right)^{*}\left(\mathbf{n}_{\vartheta}\right) Y_{l^{\prime}}^{m^{\prime}}\left(\mathbf{n}_{\vartheta^{\prime}}\right) Y_{\lambda^{\prime}}^{m-m^{\prime}}\left(\mathbf{n}_{\theta}\right)
\end{aligned}
$$

an. Die Winkel, die die Einheitsvektoren $\mathbf{n}_{\theta}, \mathbf{n}_{\vartheta}$ und $\mathbf{n}_{\vartheta^{\prime}}$ mit der Quantisierungsachse $\mathbf{e}_{3}$ einschließen, sind darin definiert durch

$$
\begin{aligned}
& \cos \theta:=\widehat{\mathbf{q}} \cdot \widehat{\mathbf{q}^{\prime}}=\frac{p^{2}-q^{2}-\frac{1}{4} q^{2}}{q q^{\prime}}, \\
& \cos \vartheta:=\frac{\frac{1}{2} q^{2}+\widehat{\mathbf{q}} \cdot \widehat{\mathbf{q}^{\prime}}}{q\left|\mathbf{q}^{\prime}+\frac{1}{2} \mathbf{q}\right|}, \quad \cos \vartheta^{\prime}:=\frac{q^{2}+\frac{1}{2} \widehat{\mathbf{q}} \cdot \widehat{\mathbf{q}^{\prime}}}{q\left|\mathbf{q}+\frac{1}{2} \mathbf{q}^{\prime}\right|},
\end{aligned}
$$

und die Azimutalwinkel aller drei Einheitsvektoren können zu Null gewählt werden.

Im Spezialfall $L=0$ wird der Kern (B.18) aufgrund der Symmetrierelationen der Clebsch-Gordan-Koeffizienten diagonal in den Indexpaaren, enthält also Faktoren $\delta_{\lambda l} \delta_{\lambda^{\prime} l^{\prime}}$. Da in der Gleichung (B.17) nur geradzahlige Drehimpulse $l, l^{\prime}$ auftreten, sind also auch $\lambda$ und $\lambda^{\prime}$ geradzahlig. Dies bedingt die feste Parität +1 unter Inversion von q, die sich auf die vollständige Wellenfunktion (B.2) überträgt. Nach der Rücktransformation in den Ortsraum, erhält man entsprechend Parität +1 unter Inversion von $\boldsymbol{\rho}$, wie auf Seite 31 behauptet.

\section{B.1.2 Unitäre Polapproximation}

Die Impulsvariable $p$ tritt auf der rechten Seite der Integralgleichung für den Partialwellenanteil der Faddeev-Komponente (B.17) nur im ersten Impulsargument der Zweiteilchen-Übergangsamplitude $t_{l}$ auf. Gelänge es, die Impulsargumente zu separieren, so könnte die Integralgleichung auf eine eindimensionale Gleichung reduziert werden. Dies wird durch die unitäre Polapproximation (UPA) erreicht, die von Lovelace [95] diskutiert wurde. Eine übersichtliche Darstellung der UPA und ihrer möglichen Erweiterungen findet sich bei Levinger [98]. Die zugrundeliegende Idee ist die Entwicklung von $t_{l}\left(p, p^{\prime} ; z\right)$ für ein festes $z=s$ nach den Eigenfunktionen $g_{n l}(p ; s)$ des Integralkerns der Lippmann-Schwinger-Gleichung (B.12), der für reelle $s<0$ bis auf eine Symmetrietransformation hermitesch ist [96, Kap. 10]. Diese reell wählbaren Eigenfunktionen, die durch den Index $n=1 \ldots N$ ( $N$ möglicherweise unendlich) abgezählt werden, erfüllen die Gleichung

$$
4 \pi \int_{0}^{\infty} \mathrm{d} p^{\prime} p^{\prime 2} v_{l}\left(p, p^{\prime}\right)\left[s-\frac{p^{2}}{2 \mu}\right]^{-1} g_{n l}\left(p^{\prime} ; s\right)=\lambda_{n l}(s) g_{n l}(p ; s), \quad s<0 .
$$


Die Eigenwerte $\lambda_{n l}(s)$ können sowohl positiv als auch negativ sein. Zu jeder physikalischen Zweiteilchen-Bindungsenergie $s=E_{n l}$ gibt es eine Eigenfunktion $g_{n l}\left(p ; E_{n l}\right)$ zum Eigenwert $\lambda_{n l}\left(E_{n l}\right)=1$, die gerade mit der Lösung $R_{n l}(p)$ des Radialteils der Zweiteilchen-Schrödinger-Gleichung über die Beziehung

$$
g_{n l}\left(p ; E_{n l}\right)=C_{n l}\left[E_{n l}-\frac{p^{2}}{2 \mu}\right] R_{n l}(p), \quad C_{n l} \in \mathbb{R}
$$

zusammenhängt. Die Funktionen $g_{n l}\left(p ; E_{n l}\right)$ werden in der Literatur gelegentlich als „Formfaktoren“ des Potentials bezeichnet. Allgemein wird die Normierung so gewählt, daß die Orthogonalitätsrelation

$$
-4 \pi \int_{0}^{\infty} \mathrm{d} p^{\prime} p^{\prime 2}\left[s-\frac{p^{2}}{2 \mu}\right]^{-1} g_{n l}\left(p^{\prime} ; s\right) g_{n^{\prime} l}\left(p^{\prime} ; s\right)=\delta_{n n^{\prime}}
$$

erfüllt ist. Die Partialwellenkomponente des Paarpotentials $v_{l}\left(p, p^{\prime}\right)$ kann daraufhin als Summe separabler Terme dargestellt werden [98].

$$
v_{l}\left(p, p^{\prime}\right)=-\sum_{n=1}^{N} \lambda_{n l}(s) g_{n l}(p ; s) g_{n l}\left(p^{\prime} ; s\right)
$$

Die UPA besteht in der Approximation von $v_{l}\left(p, p^{\prime}\right)$ durch nur einen Term:

$$
v_{l}\left(p, p^{\prime}\right) \simeq-\lambda_{n l}(s) g_{n l}(p ; s) g_{n l}\left(p^{\prime} ; s\right) .
$$

Einsetzen dieser Näherung in die Lippmann-Schwinger-Gleichung (B.12) zeigt, daß daraufhin auch $t_{l}\left(p, p^{\prime} ; z\right)$ separabel wird,

$$
t_{l}\left(p, p^{\prime} ; z\right) \simeq t_{l}^{\mathrm{UPA}}\left(p, p^{\prime} ; z\right):=-g_{n l}(p ; s) \tau_{n l}(z) g_{n l}\left(p^{\prime} ; s\right),
$$

mit der Funktion

$$
\tau_{n l}(z):=\left\{\lambda_{n l}(s)^{-1}+4 \pi \int_{0}^{\infty} \mathrm{d} p p^{2}\left[z-\frac{p^{2}}{2 \mu}\right]^{-1} g_{n l}(p ; s)^{2}\right\}^{-1} .
$$

Es bleibt die Energiegröße $s$ festzulegen. Aus der Spektraldarstellung der vollständigen Zweiteilchen-Streumatrix (B.4) ist ersichtlich, daß $t(z)$ einfache Pole bei den Zweiteilchen-Bindungsenergien $z=E_{n l}<0$ besitzt. Diese Eigenschaft überträgt sich auf die Partialwellenkomponenten $t_{l}\left(p, p^{\prime} ; z\right)$. Wählt man speziell $s=E_{n l}$ für einen bestimmten Bindungszustand $R_{n l}(p)$ und entsprechend den Formfaktor $g_{n l}\left(p ; E_{n l}\right)$ nach Gleichung (B.21) mit $\lambda_{n l}\left(E_{n l}\right)=1$, so wird dieser Pol und sein Residuum durch die UPA (B.25) reproduziert. Insbesondere für Paarpotentiale, die nur einen Bindungszustand tragen, ist daher zu erwarten, daß die UPA eine gute Approximation der Zweiteilchen-Streumatrix liefert. Diese Vorgehensweise ist eine aus der theoretischen Kernphysik bekannte Standardtechnik [98]. Eine systematische Verbesserung wird durch Einbezug weiterer Eigenfunktionen $g_{n l}(p ; s)$ in Gleichung (B.24) erreicht; diese sogenannte „unitary pole expansion“ $[98,99]$ soll hier aber nicht verfolgt werden. 


\section{B.2 Das Helium-Trimer ${ }^{4} \mathrm{He}_{3}$}

\section{B.2.1 Die Faddeev-Gleichung für das Helium-Trimer}

Der Mehrzahl der theoretischen Vorhersagen zufolge ${ }^{1}$ besitzt das Helium-Trimer nur zwei Bindungszustände, die beide zum Gesamtdrehimpuls $L=0$ gehören. Darum beschränkt sich die folgende Darstellung auf diesen einfachsten aller Fälle. Es wurde bereits bemerkt, daß der Kern $K_{\lambda l, \lambda^{\prime} l^{\prime}}^{(0)}$ (B.18) die Faktoren $\delta_{\lambda l} \delta_{\lambda^{\prime} l^{\prime}}$ enthält. Dadurch reduziert sich die Summation in der Integralgleichung für den Partialwellenanteil der Faddeev-Komponente (B.17) auf eine einfache Summation über $l^{\prime}$. Weiterhin trägt das Helium-Helium-Paarpotential nach bisherigen Erkenntnissen nur einen, äußerst schwach gebundenen Zustand zur Bindungsenergie $E_{\mathrm{g}}$ und den Quantenzahlen $n=1$, $l=0$. Es liegt daher nahe, die partielle Zweiteilchen-Streumatrix $t_{0}\left(p, p^{\prime} ; z\right)$ durch $t_{0}^{\mathrm{UPA}}\left(p, p^{\prime} ; z\right)$ (B.25) mit $s=E_{\mathrm{g}}$ zu approximieren und höhere Partialwellenanteile gänzlich zu vernachlässigen. Vom Kern verbleibt somit nur der triviale Term

$$
K_{00,00}^{(0)}\left(q, q^{\prime} ; p^{\prime}\right) \equiv 1 .
$$

Der Formfaktor $g(p):=g_{10}\left(p ; E_{\mathrm{g}}\right)$ kann nach Gleichung (B.21) direkt aus der Bindungswellenfunktion des Helium-Dimers berechnet werden und muß entsprechend der Vorschrift (B.22)

$$
-4 \pi \int_{0}^{\infty} \mathrm{d} p p^{2}\left[E_{\mathrm{g}}-\frac{p^{2}}{2 \mu}\right]^{-1} g(p)^{2}=1
$$

normiert werden. Daraufhin läßt sich die Faddeev-Komponente (B.17) als Produkt

$$
\varphi_{000}(q, p)=:-\left[E-\frac{3}{4} \frac{q^{2}}{m}-\frac{p^{2}}{m}\right]^{-1} g(p) q^{-2} A(q)
$$

ansetzen $^{2}$. Einsetzen in Gleichung (B.17) liefert eine eindimensionale Integralgleichung für die unbekannte Funktion $A(q)$,

$$
A(q)=q^{2} \tau_{0}\left(E-\frac{3}{4} \frac{q^{2}}{m}\right) \int_{0}^{\infty} \mathrm{d} q^{\prime} U\left(q, q^{\prime} ; E\right) A\left(q^{\prime}\right),
$$

mit

$$
\tau_{0}(z)=\left\{1+4 \pi \int_{0}^{\infty} \mathrm{d} p p^{2}\left[z-\frac{p^{2}}{2 \mu}\right]^{-1} g(p)^{2}\right\}^{-1} .
$$

Der symmetrische Kern der Integralgleichung (B.29) lautet

$$
U\left(q, q^{\prime} ; E\right):=-4 \pi \int_{-1}^{1} \mathrm{~d} y \frac{g\left(\sqrt{q^{\prime 2}+\frac{1}{4} q^{2}+q q^{\prime} y}\right) g\left(\sqrt{q^{2}+\frac{1}{4} q^{\prime 2}+q q^{\prime} y}\right)}{E-\left(q^{2}+q^{\prime 2}+q q^{\prime} y\right) / m},
$$

\footnotetext{
${ }^{1}$ Literaturüberblick siehe Abschnitt 3.1.3

${ }^{2}$ Der ursprünglich von Sitenko [96, Kap. 11] anstelle von Gleichung (B.28) verwendete Ansatz lautet $\varphi_{000}(q, p)=:-\left[z_{q}-p^{2} / m\right]^{-1} g(p) \tau_{0}\left(z_{q}\right) a(q)$. Die Gleichungen (B.29) und (B.31) zeigen jedoch, daß $A(q) \sim q^{2} \tau_{0}\left(z_{q}\right)$ für $q \downarrow 0$. Aus diesem Grund erscheint der Ansatz (B.28) für die numerische Behandlung von Gleichung (B.29) besser geeignet. Er geht zurück auf Nakaichi-Maeda und Lim [23].
} 
wozu die Substitution $y:=\cos \theta=\left(p^{2}-q^{2}-\frac{1}{4} q^{2}\right) / q q^{\prime}$ (B.19a) verwendet wurde. Die Funktion $A(q)$ kann also ebenfalls reell gewählt werden. Die Faddeev-Komponente $\varphi_{L M}(\mathbf{q}, \mathbf{p})$ (B.16) zu den festen Quantenzahlen $L=0, M=0$ ist dann gegeben durch

$$
\varphi_{00}(\mathbf{q}, \mathbf{p})=(4 \pi)^{-1} \varphi_{000}(q, p) .
$$

Der Vorfaktor rührt von der Funktion $\mathcal{Y}_{0000}(\hat{\mathbf{q}}, \hat{\mathbf{p}})=(4 \pi)^{-1}$ her.

\section{Numerische Lösung der Faddeev-Gleichung}

Die Integralgleichung (B.29) enthält die unbekannte Funktion $A(q)$ und die unbekannte Bindungsenergie $E$. Sie kann durch Einführung von $N_{q}$ Stützstellen $q=q_{i}$, $i=0 \ldots\left(N_{q}-1\right)$, näherungsweise in das diskrete Eigenwertproblem

$$
\mathbf{A}=\tilde{U}(E) \mathbf{A}
$$

überführt werden, wobei $\mathbf{A} \in \mathbb{R}^{N_{q}}$ ein Vektor ist, dessen Komponenten $A_{i}:=A\left(q_{i}\right)$ die gesuchte Funktion $A(q)$ approximieren und die $N_{q} \times N_{q}$-Matrix

$$
\tilde{U}(E)_{j i}=\frac{1}{2} q_{j}^{2} \tau_{0}\left(E-\frac{3}{4} \frac{q_{j}^{2}}{m}\right) U\left(q_{j}, q_{i} ; E\right) \begin{cases}q_{1}-q_{0} & : i=0 \\ q_{i+1}-q_{i-1} & : 1 \leq i \leq N_{q}-2 \\ q_{N_{q}-1}-q_{N_{q}-2} & : i=N_{q}-1\end{cases}
$$

den im einfachsten Fall linear approximierten Integralkern und die Vorfaktoren repräsentiert. Die Aufgabe besteht darin, alle Energiewerte $E$ zu finden, bei denen die Matrix $\tilde{U}(E)_{j i}$ einen Eigenwert +1 besitzt. Der zugehörige Eigenvektor ist eine der gesuchten Lösungen.

Für die Berechnung des Formfaktors $g(p)$ wurde zunächst die unter Verwendung des TTY-Potentials [54] bestimmte Bindungswellenfunktion des Helium-Dimers verwendet. Sie gehört zur Dimer-Bindungsenergie $E_{\mathrm{g}}=-0,115 \mu \mathrm{eV}$. Den Eigenwert +1 in der Eigenwertgleichung (B.33) findet man daraufhin bei den Trimer-Bindungsenergien $E=-8,88 \mu \mathrm{eV}$ und $E=-0,185 \mu \mathrm{eV}$. Der Vergleich mit den Literaturdaten in Tabelle 3.1 zeigt, daß erstere mit dem Grundzustand des Helium-Trimers identifiziert werden sollte und letztere mit dem angeregten Zustand. Die zugehörigen Eigenvektoren sind in Abbildung B.1 gegen ihren Index aufgetragen. Sie repräsentieren die Funktionen $A(q)$.

Zum Vergleich wurde dieselbe Rechnung mit dem modernen Potential von Gdanitz [66] durchgeführt. Es trägt einen gegenüber dem TTY-Potential um circa $10 \%$ geringer ausgedehnten und um circa 25\% stärker gebundenen Dimer-Bindungszustand bei der Energie $E_{\mathrm{g}}=-0,144 \mu \mathrm{eV}$. Als Bindungsenergien des Trimers erhält man $E=-9,34 \mu \mathrm{eV}$ und $E=-0,219 \mu \mathrm{eV}$.

\section{B.2.2 Die Ortsraumdarstellung der Bindungszustände}

In Kapitel 3 wird die Ortsraumdarstellung $\phi_{00}(\boldsymbol{\rho}, \mathbf{r})$ der vollständigen Bindungswellenfunktion für die beiden Zustände des Helium-Trimers benötigt. Sie kann durch 


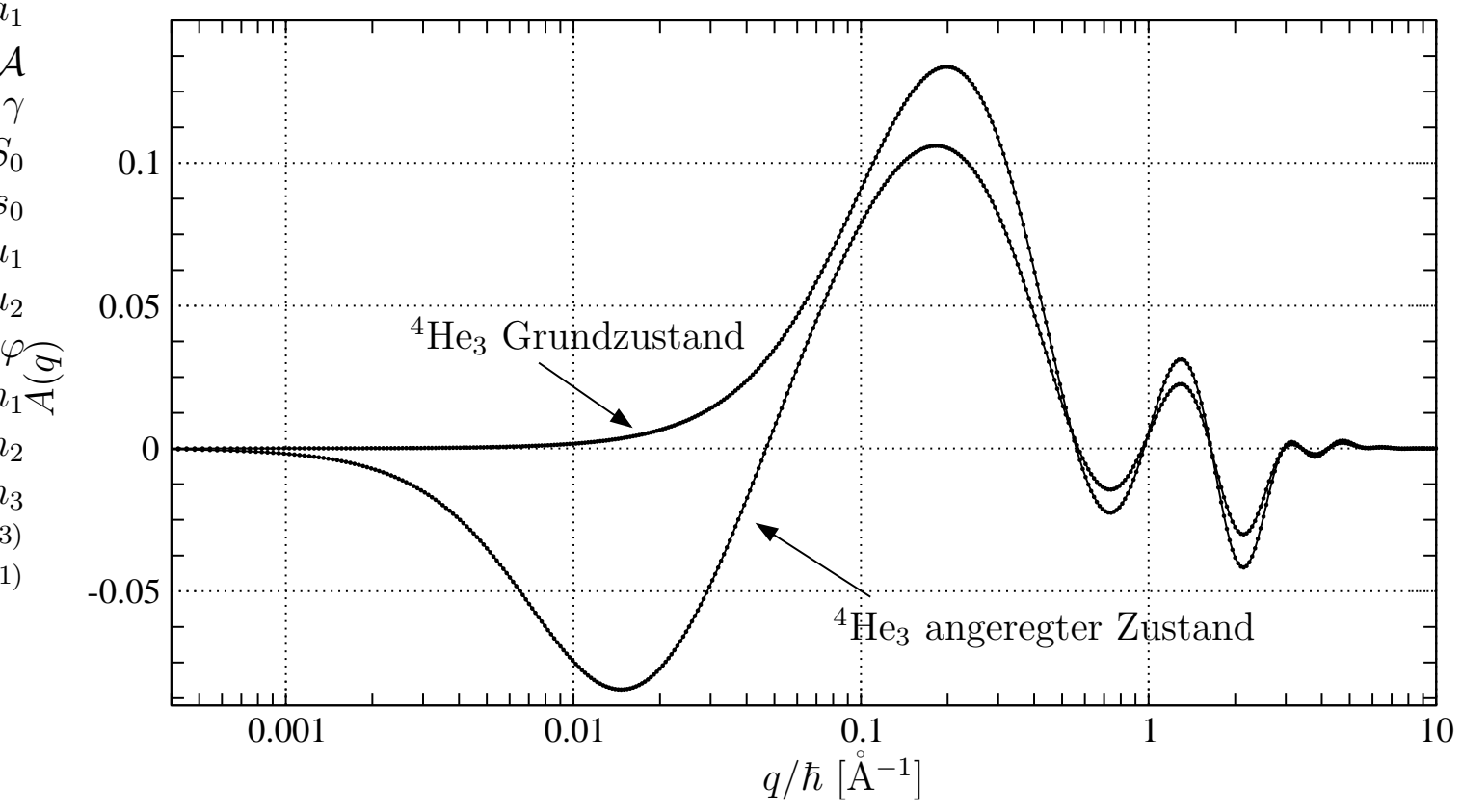

Abbildung B.1: Zwei Lösungen $A(q)$ der Gleichung (B.33) mit dem Formfaktor des TTYPotentials. Die Abszisse ist logarithmisch skaliert. Die $N_{q}=500$ Stützstellen wurden nach der Formel $q_{i}:=\alpha[\exp (\beta i)-1]-\Delta$ mit $i=0 \ldots N_{q}-1$ verteilt, wobei die Koeffizienten $\alpha, \beta$ und $\Delta$ so gewählt sind, daß die Stützstellen im Intervall $q / \hbar \in\left[10^{-5}, 7 \times 10^{2}\right] \AA^{-1}$ auf logarithmischer Skala äquidistant erscheinen.

termweise Fouriertransformation von Gleichung (B.2) berechnet werden. Die Ortsraumdarstellung der Faddeev-Komponente lautet

$$
\begin{aligned}
\varphi_{00}(\boldsymbol{\rho}, \mathbf{r}) & =(2 \pi \hbar)^{-3} \int \mathrm{d}^{3} q \int \mathrm{d}^{3} p \exp [\mathrm{i}(\mathbf{q} \cdot \boldsymbol{\rho}+\mathbf{p} \cdot \mathbf{r}) / \hbar] \varphi_{00}(\mathbf{q}, \mathbf{p}) \\
& =\left(2 \pi^{2} \hbar^{3}\right)^{-1} \int_{0}^{\infty} \mathrm{d} p p^{2} j_{0}\left(\frac{p r}{\hbar}\right) g(p) \int_{0}^{\infty} \mathrm{d} q j_{0}\left(\frac{q \rho}{\hbar}\right) \frac{A(q)}{\frac{3}{4} \frac{q^{2}}{m}+\frac{p^{2}}{m}-E} \\
& =:(4 \pi)^{-1} \varphi_{000}(\rho, r),
\end{aligned}
$$

wobei die Definition des Radialanteils $\varphi_{000}(\rho, r)$ der Faddeev-Komponente in der letzten Zeile in Analogie zu Gleichung (B.32) gewählt wurde. Die vollständige Ortsraumwellenfunktion ist nach Gleichung (B.2) gegeben durch die Kombination

$$
\phi_{00}(\boldsymbol{\rho}, \mathbf{r})=\frac{1}{N}\left\{\varphi_{00}(\boldsymbol{\rho}, \mathbf{r})+\mathcal{U}\left(\mathbb{d}^{-1}\right) \varphi_{00}(\boldsymbol{\rho}, \mathbf{r})+\mathcal{U}\left(\mathbb{d}^{-1}\right)^{2} \varphi_{00}(\boldsymbol{\rho}, \mathbf{r})\right\} .
$$

Da die Faddeev-Komponente (B.32) im Rahmen der Näherungen nur von den Beträgen ihrer Argumente abhängt, ist $\phi_{00}(\boldsymbol{\rho}, \mathbf{r})$ eine Funktion der Variablen $\rho, r$ und des Skalarprodukts $\widehat{\boldsymbol{\rho}} \cdot \widehat{\mathbf{r}}$. Dies reduziert die numerische Berechnung der Normierungskonstanten $N$ und die Überprüfung der Orthogonalität der Bindungswellenfunktionen des Grundzustandes und des angeregten Zustandes von einem sechsdimensionalen auf ein dreidimensionales Integral. Einsetzen der expliziten Darstellung der unitären Transformation (3.30) und Ausnutzen der Variablentransformation (3.27) führt auf 
die folgende Orthonormalitätsbedingung für die Ortsraum-Bindungswellenfunktionen:

$$
\begin{aligned}
\delta_{\gamma \gamma^{\prime}} & =\left\langle\phi_{\gamma} \mid \phi_{\gamma^{\prime}}\right\rangle=\int \mathrm{d}^{3} \rho \mathrm{d}^{3} r \phi_{\gamma}(\boldsymbol{\rho}, \mathbf{r}) \phi_{\gamma^{\prime}}(\boldsymbol{\rho}, \mathbf{r}) \\
& =\frac{3}{N_{\gamma} N_{\gamma}^{\prime}} \int_{0}^{\infty} \mathrm{d} \rho \rho^{2} \int_{0}^{\infty} \mathrm{d} r r^{2} \varphi_{\gamma}(\rho, r)\left[\varphi_{\gamma^{\prime}}(\rho, r)+\int_{-1}^{1} \mathrm{~d} \chi \varphi_{\gamma^{\prime}}(\xi(\rho, r, \chi), \eta(\rho, r, \chi))\right] .
\end{aligned}
$$

Hier stehen $\varphi_{\gamma}$ beziehungsweise $\varphi_{\gamma^{\prime}}$ für den in Gleichung (B.35) definierten Radialanteil der Faddeev-Komponente des Grundzustands oder des angeregten Zustands; die Funktionen

$$
\xi(\rho, r, \chi):=\sqrt{\rho^{2}+\frac{1}{4} r^{2}+\rho r \chi} \text { und } \eta(\rho, r, \chi):=\frac{1}{2} \sqrt{\rho^{2}+\frac{9}{4} r^{2}-3 \rho r \chi}
$$

enthalten die Abhängigkeit vom Winkel $\chi:=\widehat{\boldsymbol{\rho}} \cdot \widehat{\mathbf{r}}$. Die für die vorliegende Arbeit numerisch berechneten, normierten Bindungswellenfunktionen des angeregten und des Grundzustandes liefern für das Überlapp-Integral (B.37) einen Zahlenwert von $\left\langle\phi_{\mathrm{e}} \mid \phi_{\mathrm{g}}\right\rangle \approx 1,0 \times 10^{-3}$. Dies ist ein Maß für die Genauigkeit der numerischen Rechnungen.

Weiterhin können auf ähnliche Weise die winkelunabhängigen Erwartungwerte $\langle r\rangle$ und $\langle\rho\rangle$ berechnet werden. Als nützliches Ergebnis findet man beispielsweise die beiden Beziehungen zu der hypersphärischen Koordinate $R$ (3.34a),

$$
\mu_{0}\left\langle R^{2}\right\rangle=m\left\langle r^{2}\right\rangle=\frac{4}{3} m\left\langle\rho^{2}\right\rangle,
$$

und somit $\left\langle\rho^{2}\right\rangle=\frac{3}{4}\left\langle r^{2}\right\rangle$. Diese analytischen Relationen erlauben eine Kontrolle der numerisch berechneten Bindungswellenfunktionen. Ebenfalls berechnet man so die im Rahmen der Näherungen dieses Anhangs exakte Relation

$$
\left\langle\left|r_{k}\right|\right\rangle=\frac{1}{2}\langle r\rangle,
$$

die in Kapitel 3.4.2 verwendet wurde. Hier steht $r_{k}$ für eine beliebige Vektorkomponente von $\mathbf{r}$.

In Tabelle B.1 sind die aus den Bindungswellenfunktionen der beiden TrimerZustände berechneten Erwartungswerte zusammengefaßt. Aufgrund der guten Übereinstimmung mit den Literaturdaten aus Ref. [32] ist anzunehmen, daß die unitäre Polapproximation für die Bindungszustände des Helium-Trimers auch quantitativ richtige Resultate liefert. 


\begin{tabular}{|c|c|c|c|c|c|}
\hline TTY-Potential & $E_{\gamma}[\mu \mathrm{eV}]$ & $\langle r\rangle[\AA]$ & $\sqrt{\left\langle r^{2}\right\rangle}[\AA]$ & $\langle\rho\rangle[\AA]$ & $\sqrt{\left\langle\rho^{2}\right\rangle}[\AA]$ \\
\hline Grundzustand & $-8,88$ & 10,4 & 12,0 & 9,0 & 10,3 \\
\hline Angeregter Zustand & $-0,185$ & 92,7 & 114,7 & 81,9 & 97,3 \\
\hline Gdanitz-Potential & $E_{\gamma}[\mu \mathrm{eV}]$ & $\langle r\rangle[\AA]$ & $\sqrt{\left\langle r^{2}\right\rangle}[\mathrm{A}]$ & $\langle\rho\rangle[\tilde{\mathrm{A}}]$ & $\sqrt{\left\langle\rho^{2}\right\rangle}[\tilde{\mathrm{A}}]$ \\
\hline Grundzustand & $-9,34$ & 10,3 & 11,8 & 8,9 & 10,2 \\
\hline Angeregter Zustand & $-0,219$ & 88,6 & 109,7 & 78,3 & 93,1 \\
\hline
\end{tabular}

\begin{tabular}{|c|c|c|c|c|c|}
\hline Ref. [32] & $E_{\gamma}[\mu \mathrm{eV}]$ & $\langle r\rangle[\mathrm{A}]$ & $\sqrt{\left\langle r^{2}\right\rangle}[\mathrm{A}]$ & $\langle\rho\rangle[\mathrm{A}]$ & $\sqrt{\left\langle\rho^{2}\right\rangle}[\mathrm{A}]$ \\
\hline Grundzustand & $-10,89$ & 9,61 & 11,0 & 8,32 & 9,73 \\
\hline Angeregter Zustand & $-0,1962$ & 79,7 & 97,2 & 74,3 & 86,9 \\
\hline
\end{tabular}

Tabelle B.1: Erwartungswerte der Trimer-Zustände für das TTY-Potential [54] (oben) und das Potential von Gdanitz [66] (Mitte). Im Vergleich mit den Ergebnissen von Barletta und Kievsky [32] (unten) zeigt sich, daß die in der unitären Polapproximation berechneten Bindungswellenfunktionen circa zehn Prozent zu große Erwartungswerte $\langle r\rangle,\langle\rho\rangle$ liefern. Diese Abweichung liegt im Bereich der Unsicherheit verschiedener gegenwärtiger theoretischer Vorhersagen für das Helium-Trimer [32]. 


\section{Literaturverzeichnis}

[1] F. Luo, C. F. Giese und W. R. Gentry. Direct measurement of the size of the helium dimer. J. Chem. Phys. 104, 1151 (1995)

[2] W. Schöllkopf und J. P. Toennies. Nondestructive Mass Selection of Small van der Waals Clusters. Science 266, 1345 (1994)

[3] G. C. Hegerfeldt und T. Köhler. Atomic versus molecular diffraction: Influence of breakups and finite size. Phys. Rev. A 57, 2021 (1998)

[4] G. C. Hegerfeldt und T. Köhler. Deviations from classical optics in matter diffraction and determination of the size of weakly bound molecules. Phys. Rev. A 61, 23606 (2000)

[5] R. E. Grisenti, W. Schöllkopf, J. P. Toennies, G. C. Hegerfeldt, T. Köhler und M. Stoll. Determination of the Bond Length and Binding Energy of the Helium Dimer by Diffraction from a Transmission Grating. Phys. Rev. Lett. 85, 2284 (2000)

[6] K. Hornberger, S. Uttenthaler, B. Brezger, L. Hackermüller, M. Arndt und A. Zeilinger. Collisional Decoherence Observed in Matter Wave Interferometry. Phys. Rev. Lett. 90, 160401 (2003)

[7] L. de Broglie. Waves and quanta. Nature 112, 540 (1923)

[8] C. Davisson und L. H. Germer. Diffraction of Electrons by a Crystal of Nickel. Phys. Rev. 30, 705 (1927)

[9] J. Estermann und O. Stern. Beugung von Molekularstrahlen. Z. Phys. 61, 95 (1930)

[10] H. Rauch, W. Treimar und U. Bonse. Test of a Single Crystal Neutron Interferometer. Phys. Lett. 47A, 369 (1974)

[11] H. Boersch. Fresnelsche Elektronenbeugung. Naturwissenschaften 28, 709 (1940)

[12] C. Jönsson. Elektroneninterferenzen an mehreren künstlich hergestellten Feinspalten. Z. Phys. 161, 454 (1961)

[13] J. A. Leavitt und F. A. Bills. Single-Slit Diffraction Pattern of a Thermal Atomic Potassium Beam. Am. J. Phys. 37, 905 (1969) 
[14] T. A. Savas, S. N. Shah, J. M. Carter und H. I. Smith. Achromatic interferometric lithography for 100-nm-period gratings and grids. J. Vac. Sci. Technol. B 13, $2732(1995)$

[15] D. W. Keith, M. L. Schattenburg, H. I. Smith und D. E. Pritchard. Diffraction of Atoms by a Transmission Grating. Phys. Rev. Lett. 61, 1580 (1988)

[16] M. S. Chapman, C. R. Ekstrom, T. D. Hammond, R. A. Rubenstein, J. Schmiedmayer, S. Wehinger und D. E. Pritchard. Optics and Interferometry with $\mathrm{Na}_{2}$ Molecules. Phys. Rev. Lett. 74, 4783 (1995)

[17] O. Carnal und J. Mlynek. Young's Double Slit Experiment with Atoms: A Simple Atom Interferometer. Phys. Rev. Lett. 66, 2689 (1991)

[18] R. E. Grisenti, W. Schöllkopf, J. P. Toennies, G. C. Hegerfeldt und T. Köhler. Determination of Atom-Surface van der Waals Potentials from TransmissionGrating Diffraction Intensities. Phys. Rev. Lett. 83, 1755 (1999)

[19] R. Brühl, P. Fouquet, R. E. Grisenti, J. P. Toennies, G. C. Hegerfeldt, T. Köhler, M. Stoll und C. Walter. The van der Waals potential between metastable atoms and solid surfaces: Novel diffraction experiments vs. theory. Europhys. Lett. 59, 357 (2002)

[20] M. Arndt, O. Nairz, J. Vos-Andreae, C. Keller, G. van der Zouw und A. Zeilinger. Wave-particle duality of $\mathrm{C}_{60}$ molecules. Nature (London) 401, 680 (1999)

[21] T. K. Lim, S. K. Duffy und W. C. Damert. Efimov State in the ${ }^{4} \mathrm{He}$ Trimer. Phys. Rev. Lett. 38, 341 (1977)

[22] H. S. Huber und T. K. Lim. A study of the Efimov states and binding energies of the helium trimer through the Faddeev-coordinate-momentum approach. J. Chem. Phys. 68, 1006 (1977)

[23] S. Nakaichi-Maeda und T. K. Lim. Zero-energy scattering and bound states in the ${ }^{4}$ He trimer and tetramer. Phys. Rev. A 28, 692 (1983)

[24] D. V. Fedorov und A. S. Jensen. Efimov Effect in Coordinate Space Faddeev Equations. Phys. Rev. Lett. 71, 4103 (1993)

[25] E. Nielsen, D. V. Fedorov und A. S. Jensen. The structure of the atomic helium trimers: halos and Efimov states. J. Phys. B 31, 4085 (1998)

[26] A. K. Motovilov, S A. Sofianos und E. A. Kolganova. Bound states and scattering processes in the ${ }^{4} \mathrm{He}_{3}$ atomic system. Chem. Phys. Lett. 275, 168 (1997)

[27] E. A. Kolganova, A. K. Motovilov und S A. Sofianos. Three-body configuration space calculations with hard-core potentials. J. Phys. B 31, 1279 (1998)

[28] V. Roudnev und S. Yakovlev. Investigation of ${ }^{4} \mathrm{He}_{3}$ trimer on the base of Faddeev equations in configuration space. Chem. Phys. Lett. 328, 97 (2000) 
[29] A. K. Motovilov, W. Sandhas, S A. Sofianos und E. A. Kolganova. Binding energies and scattering observables in the ${ }^{4} \mathrm{He}_{3}$ atomic system. Eur. Phys. J. D 13, 33 (2001)

[30] B. D. Esry, C. D. Lin und C. H. Greene. Adiabatic hyperspherical study of the helium trimer. Phys. Rev. A 54, 394 (1996)

[31] L. W. Bruch. Boson trimers with nonzero total angular momenta. J. Chem. Phys. 110, 2410 (1999)

[32] P. Barletta und A. Kievsky. Variational description of the helium trimer using correlated hyperspherical harmonic basis functions. Phys. Rev. A 64, 042514 (2001)

[33] M. Lewerenz. Structure and energetics of small helium clusters: Quantum simulations using a recent perturbational pair potenial. J. Chem. Phys. 106, 4596 (1997)

[34] I. Røeggen und J. Almlöf. An accurate calculation of the three-body potential for the ground state of the helium trimer. J. Chem. Phys. 102, 7095 (1995)

[35] E. Braaten und H.-W. Hammer. Universality in the three-body problem for ${ }^{4} \mathrm{He}$ atoms. Phys. Rev. A 67, 042706 (2003)

[36] E. Braaten, H.-W. Hammer und M. Kusunoki. Universal equation for Efimov states. Phys. Rev. A 67, 022505 (2003)

[37] M. T. Yamashita, R. S. Marques de Carvalho, Lauro Tomio und T. Frederico. Scaling predictions for radii of weakly bound triatomic molecules. Phys. Rev. A 68, 012506 (2003)

[38] V. Efimov. Energy levels arising from resonant two-body forces in a three-body system. Phys. Lett. 33B, 563 (1970)

[39] E. Braaten, H.-W. Hammer und M. Kusunoki. Efimov States in a Bose-EinsteinCondensate near a Feshbach Resonance. Phys. Rev. Lett. 90, 170402 (2003)

[40] T. Köhler. Molekülbeugung an mikrofabrizierten Strukturen. Dissertation, Universität Göttingen (1998)

[41] A. R. W. McKellar. Infrared spectra of hydrogen dimers. J. Chem. Phys. 92, 3261 (1990)

[42] J. R. Taylor. Scattering Theory (John Wiley \& Sons, 1972)

[43] D. Colton und R. Kress. Integral equation methods in scattering theory (John Wiley \& Sons, Inc., 1983)

[44] J. Meixner und F. W. Schäfke. Mathieusche Funktionen und Sphäroidenfunktionen (Springer, Berlin, 1954) 
[45] R. G. Newton. Scattering Theory of Waves and Particles (McGraw-Hill, 1966)

[46] C. J. Joachain. Quantum Collision Theory (North-Holland Physics Publishing, 1975)

[47] M. Born und E. Wolf. Principles of Optics (Pergamon Press, 1959)

[48] T. D. Klots, Carl Chuang, R. S. Ruoff, T. Emilsson und H. S. Gutowsky. Rotational spectra and structures of the $\mathrm{Ar}_{2}-\mathrm{H}^{35} \mathrm{Cl} /{ }^{37} \mathrm{Cl}$ trimers. J. Chem. Phys. 86, 5315 (1987)

[49] L. D. Faddeev. Mathematical Aspects of the Three-Body Problem in the Quantum Scattering Theory (Sivan, Jerusalem, 1965)

[50] A. R. Janzen und R. A. Aziz. Modern He-He potentials: Another look at binding energy, effective range theory, retardation, and Efimov states. J. Chem. Phys. 103, $9626(1995)$

[51] T. González-Lezana, J. Rubayo-Soneira, S. Miret-Artés, F. A. Gianturco, D. Delgado-Barrio und P. Villareal. Efimov States for ${ }^{4} \mathrm{He}$ Trimers? Phys. Rev. Lett. 1648, 82 (1999)

[52] T. González-Lezana, D. Lopez, S. Miret-Artés, F. A. Gianturco, D. DelgadoBarrio und P. Villareal. Rotation-vibration interaction in ${ }^{4} \mathrm{He}$ trimers. Chem. Phys. Lett. 105, 335 (2001)

[53] T. G. Lee, B. D. Esry, Bing-Cong Gou und C. D. Lin. The helium trimer has no bound rotational excited states. J. Phys. B 34, 203 (2001)

[54] K. T. Tang, J. P. Toennies und C. L. Yiu. Accurate Analytical He-He van der Waals Potential Based on Perturbation Theory. Phys. Rev. Lett. 74, 1546 (1995)

[55] E. O. Alt, P. Grassberger und W. Sandhas. Reduction of the three-particle collision problem to multi-channel two-particle Lippmann-Schwinger equations. Nucl. Phys. B 2, 167 (1967)

[56] W. Schöllkopf. Beugung von Atom-, Molekular- und Clusterstrahlen an nanostrukturierten Transmissionsgittern. Dissertation, Universität Göttingen (1998)

[57] L. W. Bruch, W. Schöllkopf und J. P. Toennies. The formation of dimers and trimers in free jet ${ }^{4} \mathrm{He}$ cryogenic expansions. J. Chem. Phys. 117, 1544 (2002)

[58] R. E. Grisenti, W. Schöllkopf, J. P. Toennies, J. R. Manson, T. A. Savas und H. I. Smith. He-atom diffraction from nanostructure transmission gratings: The role of imperfections. Phys. Rev. A 61, 033608 (2000)

[59] L. W. Bruch und Ar. Abanov. Asymptotic speed ratio in a free helium jet. J. Chem. Phys. 115, 10261 (2001) 
[60] F. Constantinescu. Distributionen und ihre Anwendungen in der Physik (Teubner, 1974)

[61] R. D. McCarty. Thermodynamic Properties of Helium 4 from 2 to $1500 \mathrm{~K}$ at Pressures to $10^{8}$ Pa. J. Phys. Chem. Ref. Data 2, 923-1042 (1973)

[62] K. Winkelmann. Rotational relaxation of para- and normal-hydrogen in free jet expansions. In R. Camparque (Hg.), Rarefied Gas Dynamics, Bd. 2, S. 899 (CEA, Paris, 1979)

[63] G. C. Hegerfeldt und T. Köhler. How to Study the Elusive Efimov State of the ${ }^{4} \mathrm{He}_{3}$ Molecule through a New Atom-Optical State-Selection Technique. Phys. Rev. Lett. 84, 3215 (2000)

[64] F. Luo, G. Kim, G. C. McBane, C. F. Giese und W. R. Gentry. Influence of retardation on the vibrational wave function and binding energy of the helium dimer. J. Chem. Phys. 98, 9687 (1993)

[65] A. R. Janzen und R. A. Aziz. An accurate potential energy curve for helium based on ab inito calculations. J. Chem. Phys. 107, 914 (1997)

[66] R. J. Gdanitz. Accurately solving the electronic Schrödinger equation of atoms and molecules using explicitly correlated $\left(r_{12}\right) M R-C I$. VI. The helium dimer $\left(\mathrm{He}_{2}\right)$ revisited. Mol. Phys. 99, 923 (2001)

[67] J. M. Blatt und V. F. Weisskopf. Theoretical Nuclear Physics (Springer, New York, 1979)

[68] J. Lekner. Critical binding of diatomic molecules. Mol. Phys. 23, 619 (1972)

[69] M. Boustimi, J. Baudon, M. Ducloy, J. Reinhardt, F. Perales, C. Mainos, V. Bocvarski und J. Robert. Metastable rare gas atoms scattered by nano- and micro-slit transmission gratings. Eur. Phys. J. D 17, 141 (2001)

[70] M. Boustimi, J. Baudon, F. Pirani, M. Ducloy, J. Reinhardt, F. Perales, C. Mainos, V. Bocvarski und J. Robert. Surface-induced vibrational transition of metastable nitrogen molecules passing through a nano-slit grating. Europhys. Lett. 56, $644(2001)$

[71] J. C. Karam, M. Boustimi, J. Baudon, M. Ducloy, F. Perales, J. Reinhardt, V. Bocvarski, C. Mainos, F. Levron und J. Robert. Endothermal fine-structure transition of metastable argon atoms passing through a micro-slit copper grating. Europhys. Lett. 60, 207 (2002)

[72] M. Stoll und T. Köhler. Inelastic Diffraction and Spectroscopy of Very Weakly Bound Clusters. J. Phys. B 35, 4999 (2002)

[73] G. Danby. Theoretical studies of van der Waals molecules: the $\mathrm{H}_{2}-\mathrm{H}_{2}$ dimer. J. Phys. B 16, 3411 (1983) 
[74] G. Danby. Theoretical studies of van der Waals molecules: the $\mathrm{D}_{2}-\mathrm{D}_{2}$ dimer. J. Phys. B 22, 1785 (1989)

[75] A. R. W. McKellar und H. L. Welsh. Spectra of $\left(\mathrm{H}_{2}\right)_{2},\left(\mathrm{D}_{2}\right)_{2}$, and $\mathrm{H}_{2}-\mathrm{D}_{2}$ Van der Waals Complexes. Can. J. Phys. 52, 1082 (1974)

[76] A. R. W. McKellar und J. Schaefer. Far-infrared spectra of hydrogen dimers: Comparisons of experiment and theory for $\left(\mathrm{H}_{2}\right)_{2}$ and $\left(\mathrm{D}_{2}\right)_{2}$ at $20 \mathrm{~K}$. J. Chem. Phys. 95, 3081 (1991)

[77] K. B. Whaley, C. Yu, C. S. Hogg, J. C. Light und S. J. Sibener. Investigation of the spatially anisotropic component of the laterally averaged molecular hydrogen/Ag(111) physisorption potential. J. Chem. Phys. 83, 4235 (1985)

[78] K. Kern, R. David und G. Comsa. Low temperature measurements of the rotational relaxation in $\mathrm{D}_{2}$ free jets. J. Chem. Phys. 82, 5673 (1985)

[79] D. R. Miller. Free Jet Sources. In G. Scoles (Hg.), Atomic and Molecular Beam Methods (Oxford University Press, New York, 1988)

[80] M. J. Norman, R. O. Watts und U. Buck. A spherical potential for hydrogen from solid state and scattering data. J. Chem. Phys. 81, 3500 (1984)

[81] J. Schaefer. Empirical corrections of the rigid rotor interaction potential of $\mathrm{H}_{2}-\mathrm{H}_{2}$ in the attractive region: dimer features in the FIR absorption spectra. Astron. Astrophys. 284, 1015 (1994)

[82] P. Diep und J. K. Johnson. An accurate $\mathrm{H}_{2}-\mathrm{H}_{2}$ interaction potential from first principles. J. Chem. Phys. 112, 4465 (2000). Erratum: J. Chem. Phys. 112, 4465 (2000)

[83] H. Hoinkes. The physical interaction potential of gas atoms with single-crystal surfaces, determined from gas-surface diffraction experiments. Rev. Mod. Phys. 52, $933(1980)$

[84] S. Nakaichi, T. K. Lim, Y. Akaishi und H. Tanaka. ${ }^{4}$ He tetramer and the Faddeev-Yakubovsky theory. Phys. Rev. A 26, 32 (1982)

[85] I. N. Filikhin, S. L. Yakovlev, V. A. Roudnev und B. Vlahovic. The ${ }^{4}$ He tetramer ground state in the Faddeev-Yakubovsky differential equations formalism. J. Phys. B 35(3), 501 (2002)

[86] J. P. Toennies, R. Brühl, A. Kalinin und O. Kornilov. Gitterbeugung von kleinen Heliumclustern: Evidenz für magische Zahlen N=10, 15, 26, 44 und 85. Verhandlungen der DPG (2003). Poster A 11.36

[87] J. Baudon, R. Mathevet und J. Robert. Atomic interferometry. J. Phys. B 32, R173 (1999) 
[88] B. Brezger, L. Hackermüller, S. Uttenthaler, J. Petschinka, M. Arndt und A. Zeilinger. Matter Wave Interferometer for Large Molecules. Phys. Rev. Lett. 88, $100404(2002)$

[89] R. Brühl, O. Kornilov und J. P. Toennies. Erste Messungen mit einem universellen Materiewellen-Interferometer. Verhandlungen der DPG (2002). Poster Q 301.18

[90] C. P. Walter. Diffraction of Metastable Rare-Gas Atoms from Nanostructured Transmission Gratings. Dissertation, Universität Göttingen (2002)

[91] F. Oberhettinger. Tabellen der Fourier Transformation (Springer, Berlin, 1957)

[92] M. Abramowitz und I. A. Stegun (Hg.). Handbook of Mathematical Functions (Dover Publications, 1968)

[93] A. M. Marvin und F. Toigo. van der Waals interaction between a point particle and a metallic surface. I. Theory. Phys. Rev. A 25, 782 (1982)

[94] L. D. Faddeev. Scattering theory for a 3-particle system. Soviet Phys. - JETP 12, $1014(1961)$

[95] C. Lovelace. Practical Theory of Three-Particle States. I. Nonrelativistic. Phys. Rev. 135, B 1225 (1964)

[96] A. G. Sitenko. Lectures in Scattering Theory (Pergamon Press, 1971)

[97] M. E. Rose. Elementary Theory of Angular Momentum (John Wiley \& Sons, 1957)

[98] J. S. Levinger. The Two and Three Body Problem. In Nuclear Physics, Bd. 71 von Springer Tracts in Modern Physics, S. 88-240 (Springer, Berlin, 1974)

[99] E. Harms. Convenient Expansion for Local Potentials. Phys. Rev. C 1, 1667 (1970) 


\section{Danksagung}

An dieser Stelle möchte ich Prof. Dr. Gerhard C. Hegerfeldt herzlich für die Betreuung und Unterstützung meiner Promotion danken. In unseren zahllosen, angenehmen Diskussionen habe ich oftmals einen neuen Blickwinkel und weiterführende Denkanstöße gewonnen.

Sehr verbunden bin ich ebenfalls Prof. Dr. J. Peter Toennies, Dr. Rüdiger Brühl, Anton Kalinin und Oleg Kornilov vom Max-Planck-Institut für Strömungsforschung; die enge Zusammenarbeit mit dem Experiment empfand ich als eine sehr wichtige Bereicherung. Meinen ehemaligen Kollegen Dr. Thorsten Köhler und Dr. Christian P. Walter vom Institut für Theoretische Physik der Universität Göttingen danke ich für den regen fachlichen Austausch.

Prof. Dr. Kurt Schönhammer danke ich für die Übernahme des Korreferats. Matthias Küntzel bin ich sehr dankbar für das geduldige Korrekturlesen dieser Arbeit; verbliebene Fehler sind selbstverständlich meine eigenen.

Die Finanzierung meiner Promotion wurde großenteils durch ein Georg-Christoph-Lichtenberg-Stipendium des Landes Niedersachsen im Rahmen der Göttingen Graduate School of Physics ermöglicht.

Meinen guten Eltern und Geschwistern und der Familie bin ich für ihre Unterstützung verpflichtet. Nicht zuletzt danke ich meiner allerliebsten Iris. 


\section{Lebenslauf}

NAME:

Geburtsdatum:

Geburtsort:

STAATSANGEHÖRIGKEIT:
Werner Martin Stoll

13.09.1974

Peine

deutsch

\section{Werdegang}

- 1981-1994 Schulbesuch in Peine. 1994 Abitur am Gymnasium am Silberkamp.

- 1994-1995 Zivildienst in Peine.

- 1995-1997 Studium der Physik an der Universität Bremen. 1997 Vordiplom Physik.

- 1997-1998 Studium der Physik und Mathematik an der Universität Edinburgh, Schottland, Großbritannien.

- 1998-2000 Studium der Physik an der Universität Göttingen. 11.1999-11.2000 Diplomarbeit am Institut für Theoretische Physik, Arbeitsgruppe Prof. Dr. G. C. Hegerfeldt. 11.2000 Diplom Physik.

- 12.2000-12.2003 Promotionsstudiengang Physik an der Universität Göttingen am Institut für Theoretische Physik, Arbeitsgruppe Prof. Dr. G. C. Hegerfeldt. 